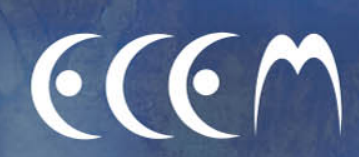

\title{
Abstracts
}

Edited by

Ulrich Ansorge, Thomas Ditye, Arnd Florack, and Helmut Leder

$1^{\text {th }}$ European Conference on Eye Movements August 16-21, 2015 | University of Vienna, Austria 


\section{ECEM 2015}

\section{Abstracts}

\section{of the $18^{\text {th }}$ European Conference on Eye Movements}

Edited by Ulrich Ansorge, Thomas Ditye, Arnd Florack, and Helmut Leder

August $16^{\text {th }}$ to $21^{\text {st }}, 2015$

Vienna, Austria

- online version, updated August 21, 2015- 
The background of the front cover image depicts a ceiling painting of the Main Ceremonial Chamber (Großer Festsaal) of the University of Vienna. You will see it when you look up during ECEM 2015's poster sessions.

The painting is called "The Triumph of Light over Darkness". It was created by Franz Matsch. In 1894, the Ministry of Education commissioned Matsch - together with Gustav Klimt - to create a set of paintings representing the different faculties. Klimt was commisioned to paint Medicine, Jurisprudence and Philosophy, while Matsch was assigned Theology. Klimt's faculty paintings faced severe criticism. They were considered to be too avantgarde and incompatible with a more conservative tradition. Klimt ultimately withdrew his works, and they were destroyed by fire in 1945.

The photography of Matsch's painting is used with kind permission of the University of Vienna. A heatmap of artificial data in the shape of the ECEM logo has been superimposed. Idea and realization: Christian Valuch, University of Vienna, 2015. 


\section{Contents}

Keynotes 9

Sunday, August $16^{\text {th }}, 6.30 \mathrm{pm}-7.30 \mathrm{pm} \ldots \ldots \ldots \ldots \ldots \ldots$

Peter König . . . . . . . . . . . . . . . . . . . . . . . . . . . . . . . . . . . .

Monday, August $17^{\text {th }}, 11.00 \mathrm{am}-12.00 \mathrm{pm} \ldots \ldots \ldots \ldots \ldots \ldots$. . . . . . . . . . . . . . . . . . .

John K. Tsotsos . . . . . . . . . . . . . . . . . . . . . . . 12

Tuesday, August $18^{\text {th }}, 11.00$ am $-12.00 \mathrm{pm} \ldots \ldots \ldots \ldots \ldots \ldots$. . . . . . . . . . . . . . . . .

Robin Walker . . . . . . . . . . . . . . . . . . . . . . 14

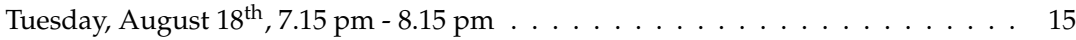

Françoise Vitu . . . . . . . . . . . . . . . . . . . . . . 15

Wednesday, August $19^{\text {th }}, 11.00 \mathrm{am}-12.00 \mathrm{pm} \ldots \ldots \ldots \ldots \ldots \ldots$

Jukka Hyönä ． . . . . . . . . . . . . . . . . . . . . . . . 17

Thursday, August $20^{\text {th }}, 11.00 \mathrm{am}-12.00 \mathrm{pm} \ldots \ldots \ldots \ldots \ldots$

Laurent Itti . . . . . . . . . . . . . . . . . . . . . . . . . . . . . . 19

Friday, August $21^{\text {st }}, 11.30 \mathrm{am}-12.30 \mathrm{pm} \ldots \ldots \ldots \ldots$. . . . . . . . . . 20

Tirin Moore . . . . . . . . . . . . . . . . . . . . 20

Talks 21

Monday, August $17^{\mathrm{th}}, 8.50 \mathrm{am}-10.30 \mathrm{am} \ldots \ldots \ldots \ldots 22$

Reading: Individual differences . . . . . . . . . . . . . . . . . . . 22

Integrating eye movements in research on expertise and skill acquisition . . . 25

Eye behavior: Perceptual effects and cognitive influences . . . . . . . . . . . . 28

Eye movements: Smooth pursuit, manual tracking, control . . . . . . . . . . . . 31

Monday, August $17^{\text {th }}, 1.20 \mathrm{pm}-3.00 \mathrm{pm} \ldots \ldots \ldots \ldots \ldots$. . . . . . . . . . . . . . . . . . . .

Reading: General I . . . . . . . . . . . . . . . . . . . . . . . . 34

Eye movements and reading: Expertise . . . . . . . . . . . . . . . . . . . 37

Eye movements: Social influences . . . . . . . . . . . . . . . . . . . . . . . . . . . . . . . . . . . . . .

Microsaccades . . . . . . . . . . . . . . . . . . 43

Monday, August $17^{\text {th }}, 5.00 \mathrm{pm}-6.40 / 7.00 \mathrm{pm} \ldots \ldots \ldots \ldots \ldots$. . . . . . . . . . . . . . . 46

Reading: Chinese . . . . . . . . . . . . . . . . . . . 46

Visual search . . . . . . . . . . . . . . . . . . . . . . . . . . . 49

Eye movements and reading: Clinical . . . . . . . . . . . . . . . . . . 52

Eye movements: Conflict and inhibition . . . . . . . . . . . . . . . . . . . . . 55

Tuesday, August $18^{\text {th }}, 8.50 / 9.10 \mathrm{am}-10.30 \mathrm{am} \ldots \ldots \ldots \ldots$

What the eyes tell us about bilingual language processing . . . . . . . . . . . . . 58

Natural scene perception and eye-tracking . . . . . . . . . . . . . . . 61

Saccades I . . . . . . . . . . . . . . . . . . . . . . . . . . . 64

Eye movements and reading: Influences of higher cognition . . . . . . . . . 66 


\section{Contents}

Tuesday, August $18^{\text {th }}, 1.20 \mathrm{pm}-3.00 / 3.20 \mathrm{pm} \ldots \ldots \ldots \ldots$. . . . . . . . . . 68

Reading: Development and special populations . . . . . . . . . . . . . . 68

Neural mechanisms of saliency, attention and orienting . . . . . . . . . . 72

Eye movements: Neurophysiology and neuropsychology . . . . . . . . . . . . . 75

Reading: Dyslexia and aphasia . . . . . . . . . . . . . . . . 78

Tuesday, August $18^{\text {th }}, 5.00 \mathrm{pm}-6.40 \mathrm{pm} \ldots \ldots \ldots \ldots$. . . . . . . . . . . . . . . 81

Reading: Influences of context I . . . . . . . . . . . . . . . . . . . . . 81

Reading: Influences of valence, emotion, and negation . . . . . . . . . . . . . 84

Eye movements: Neurophysiology and EEG . . . . . . . . . . . . . . . . . . . . . . 87

The COGAIN Eye Data Quality Project . . . . . . . . . . . . . . . . . . . . . . . . . . . . 90

Wednesday, August $19^{\text {th }}, 8.50 \mathrm{am}-10.30 \mathrm{am} \ldots \ldots \ldots \ldots$. . . . . . . . . . . 94

In memory of Keith Rayner . . . . . . . . . . . . . . . . . . . . . . . . . . 94

Eye movements in new media and film . . . . . . . . . . . . . . . . 97

Reading: Influences of context II . . . . . . . . . . . . . . . . . . 100

Saccades and perception . . . . . . . . . . . . . . . . . . 103

Thursday, August $20^{\text {th }}, 8.50$ am -10.30 am $\ldots \ldots \ldots \ldots$. . . . . . . . 106

Triple P: Pre-, peri- and post-saccadic perception . . . . . . . . . . . . . . . 106

Reading: General II . . . . . . . . . . . . . . . . . . . . . . . . . . . . . . . . . 109

Eye movements and attention . . . . . . . . . . . . . . . . . . . . . . 112

Mobile eye-tracking: Tools and findings . . . . . . . . . . . . . . . . . . . . 115

Thursday, August $20^{\text {th }}, 1.20 \mathrm{pm}-3.00 \mathrm{pm} \ldots \ldots \ldots \ldots$. . . . . . . . . 117

Development of parafoveal processing during reading acquisition . . . . . . . 117

Eye movements and attention in moving images . . . . . . . . . . . . . . . . 120

Eye movements, attention, and memory . . . . . . . . . . . . . . . . . . . . 123

Eye movements: Faces . . . . . . . . . . . . . . . . . . . . . 126

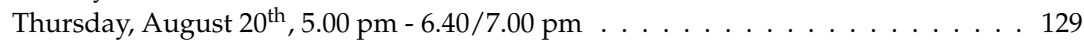

Reading: Parafoveal processing . . . . . . . . . . . . . . . . . . . . . . . . . 129

Saccades II . . . . . . . . . . . . . . . . . . . . . . . . . . . . 132

Scanpaths, saliency, and fixation memory . . . . . . . . . . . . . . . . . . . 135

Eye-tracking: Issues of measurement . . . . . . . . . . . . . . . . . . 138

Friday, August $21^{\text {st }}, 8.50 \mathrm{am}-10.30 / 10.50 \mathrm{am} \ldots \ldots \ldots$. . . . . . . . . . . 141

Eye tracking in visual aesthetics: Perception of art . . . . . . . . . . . . . . 141

Investigating deficit theories of dyslexia with naturalistic reading paradigms using eye-movement recordings . . . . . . . . . . . . 143

Eye movements: Clinical . . . . . . . . . . . . . . . . . . . . . . . . . . . . 146

Eye movements: Applied questions . . . . . . . . . . . . . . . . . . . . . 149

Posters 153

Monday, August $17^{\text {th }}, 3.00 \mathrm{pm}-5.00 \mathrm{pm} \ldots \ldots \ldots \ldots \ldots$. . . . . . . . . . . . . . . . . . . . . . . . . . . . . . .

Cognitive influences . . . . . . . . . . . . . . . . . . . . . 154

Looking at text and pictures . . . . . . . . . . . . . . . . 162

Reading . . . . . . . . . . . . . . . . . . . . 165

Saccades . . . . . . . . . . . . . . . . . . . . . 171

Smooth pursuit . . . . . . . . . . . . . . . . . . . . 180

Vergence . . . . . . . . . . . . . . . . . . . . . . . 185

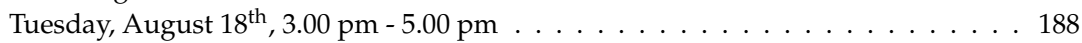

Curvature of eye movement trajectories . . . . . . . . . . . . . . . 188

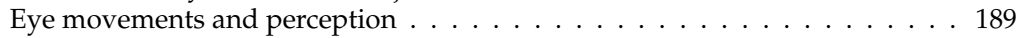

Eye-tracking methods . . . . . . . . . . . . . . . . . 193

Microsaccades . . . . . . . . . . . . . . . . . . . . . . . . 198 
Reading . . . . . . . . . . . . . . . . . . . . . . . . . . 199

Social influences . . . . . . . . . . . . . . . . . . . . . 206

Visual influences . . . . . . . . . . . . . . . . . . . . . . 207

Visual search . . . . . . . . . . . . . . . . . . . . . . . . . . . . 213

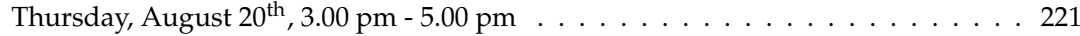

Attention capture/Oculomotor capture . . . . . . . . . . . . . . . . . 221

Clinical applications . . . . . . . . . . . . . . . . . . . . 225

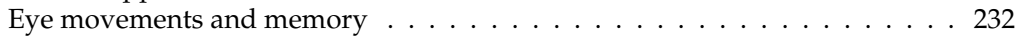

Intermodal influences . . . . . . . . . . . . . . . . . . . . . 237

Learning and adaptation . . . . . . . . . . . . . . . . . . . . . . . . . . . . . . . . . . . . . . . . .

Reading . . . . . . . . . . . . . . . . . . . . . 246

Social influences . . . . . . . . . . . . . . . . . . . . . . . 252

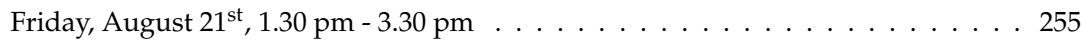

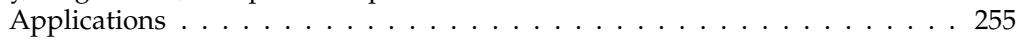

Biological influences . . . . . . . . . . . . . . . . . . . . . . 267

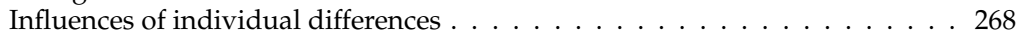

Parafoveal processing . . . . . . . . . . . . . . . . . . . . . 275

Reading . . . . . . . . . . . . . . . . . . . . . 277

Scan paths and scan patterns . . . . . . . . . . . . . . 282

$\begin{array}{ll}\text { Author Index } & 285\end{array}$

\section{Note}

Keynote, talk, and poster abstracts are listed separately in the chronological order at the conference. Simultaneous sessions are ordered by their location (HS 7, HS 30, HS 31, HS 32). 


\section{Keynotes}


Keynotes

\title{
Sunday, August $16^{\text {th }}, 6.30 \mathrm{pm}-7.30 \mathrm{pm}$
}

\author{
Room: Audimax
}

Eye movements as a central part of cognitive processes

\author{
Peter König \\ University Osnabrück, Germany \\ pkoenig@uos.de
}

Humans make billions of eye movements in their lifetime that intimately relate action and perception. Each of these entails a decision of how long to linger at a fixated location and where to look next.

Based on recent work we can predict location as well as duration of fixational eye movements to a substantial degree. Furthermore, we dissect the contribution and dynamic interactions of stimulus dependent properties as captured by the concepts of saliency map and hazard functions, geometrical constraints like spatial bias and saccadic momentum and task dependent aspects.

Each eye movement scrutinizes part of the visual field, visible before the eye movement at low spatial resolution, in detail and refining predictions about the world. This allows an investigation of neural activity compatible or violating active predictions, learned from the contingencies between actions and the consequent changes in sensory input. Specifically, error signals to saccade-contingent changes of veridical and inferred inputs by contrasting the EEG activity after saccades to a stimulus presented inside or outside the blind-spot. We can demonstrate predictive signals across multiple levels of the visual hierarchy, based on generative models that differentiate between signals that originate from the outside world and those that are inferred.

Finally we address the apparent conflict between the central role of eye-movements and attention for cognition and the seemingly specialized and sparse network of brain structures related to eye movements. Indeed, in previous work the involvement of the medial temporal lobe of macaque monkey in overt attention has been shown. Here we demonstrate that movement of covert attention, without any physical movement, also elicits periodic spatial receptive fields with a triangular tiling of the space in a significant population $(20 / 141,14 \%$ neurons at a FDR $<5 \%$ ) of entorhinal grid cells. These results provide compelling evidence that grid cells in macaque monkeys do not rely on physical movement and make a general contribution to mental operations.

These results demonstrate the close interaction of eye-movements, attention and general cognition and advertise the investigation of eye-movements as a window to cognition.

\section{About the speaker}

Peter König studied physics and medicine in Bonn and obtained a doctorate of medicine in 1990 from the University of Würzburg, Germany. After a three-year post-doc time in the lab of Wolf Singer at the MPI for brain research in Frankfurt, Dr. König worked as a senior research fellow at the Neurosciences Institute in La Jolla, California. Later, he moved to Switzerland to work at the ETH Zürich before he became head of the Neurobiopsychology research group at the Institute of Cognitive Science at the University of Osnabrück (Germany) in 2003. Since 2005, Dr. König is also head of the Institute of Cognitive Science at the University of Osnabrück. 
In his research, Dr. König aims to investigate the neurophysiological basis of cognitive functions. He uses experimental and theoretical approaches to study sensory processing and sensory motor integration in the mammalian cortex under natural conditions. Emphasis is placed on the role of top-down signals, their relation to the fast dynamics, learning and plasticity in the neuronal network. Insights obtained from this work are transferred to real-world applications. 
Keynotes

\title{
Monday, August $17^{\text {th }}, 11.00$ am $-12.00 \mathrm{pm}$
}

\author{
Room: Audimax
}

\section{STAR: The Selective Tuning Attentive Reference Model}

\author{
John K. Tsotsos \\ Dept. of Electrical Engineering and Computer Science, York University, Toronto, Canada \\ tsotsos@cse.yorku.ca
}

The Selective Tuning Attentive Reference model (STAR) is previewed (Tsotsos 2011, Tsotsos \& Kruijne 2014). STAR, currently in its early stages of development, integrates proposals for an executive controller, working memory, attentional mechanisms, eye movements, visual processing and the communication among these that is hoped to enable flexible and generalizable visual task execution. This presentation will focus on the model elements that concern fixation change. Differing from most other models where a representation of image saliency drives selection of gaze patterns supporting a fixate-and-recognize vision process, here, saliency plays multiple roles all different from this common role. The first is stimulusdriven local feature conspicuity (stimulus-based attentional push), restricted to the visual periphery and enabling fixation changes overtly for reasons of surprise, novelty and exploration, based on the AIM framework (Bruce \& Tsotsos 2009). Another is an object-centred conspicuity to drive central visual field fixation changes. These changes may be covert or short-range overt, and are intended to examine object components for purposes such as description, comparison or discrimination as well as pursuit. The central-peripheral distinction is imposed not only because of the retinal receptor anisotropy, but also to solve the boundary problem present in layered hierarchical representations (Tsotsos 2011). A third representation is task-specific attentional pull. This represents priority or urgency (conspicuity among unfinished components in the task domain) to attend a particular location, feature or object related to current task. Cognitive Programs lay out the temporal and causal sequence of operations comprising a task (Tsotsos \& Kruijne 2014), working memory stores completed task elements, and the disparity at a given time between program and memory lead to task element priority. The latest results from the first stages of experimentation with STAR will round out the presentation.

\section{References}

Bruce, N.D.B., Tsotsos, J.K. (2009). Saliency, Attention, and Visual Search: An Information Theoretic Approach, J. of Vision, 9:3, p1-24.

Tsotsos, J.K. (2011). A Computational Perspective on Visual Attention, The MIT Press.

Tsotsos, J.K., Kruijne W. (2014).Cognitive programs: Software for attention's executive, Frontiers in Psychology: Cognition 5:1260.

\section{About the speaker}

John K. Tsotsos is Distinguished Research Professor of Vision Science at York University. He is Director of the Centre for Innovation in Computing at Lassonde, Canada Research Chair in Computational Vision, and is a Fellow of the Royal Society of Canada.

He received his doctorate in Computer Science from the University of Toronto. He did a postdoctoral fellowship in Cardiology at Toronto General Hospital and then joined the University of Toronto on faculty in both Computer Science and in Medicine, where he stayed 
for 20 years, 10 of which were as a Fellow of the Canadian Institute for Advanced Research. He then moved to York University serving as Director of the Centre for Vision Research for 7 years. Visiting positions were held at the University of Hamburg, Polytechnical University of Crete, Center for Advanced Studies at IBM Canada, INRIA Sophia-Antipolis, and the Massachusetts Institute of Technology.

He has published in computer science, neuroscience, psychology, robotics and bio-medicine. Current research has a main focus in developing a comprehensive theory of visual attention in humans. A practical outlet for this theory forms a second focus, embodying elements of the theory into the vision systems of mobile robots. 
Keynotes

\title{
Tuesday, August $18^{\text {th }}, 11.00$ am $-12.00 \mathrm{pm}$
}

\author{
Room: Audimax
}

\section{Saccadic distractor effects: The when and where of saccade generation}

\author{
Robin Walker \\ Royal Holloway, University of London, United Kingdom \\ Robin.Walker@rhul.ac.uk
}

A great deal has been learned about the control of saccades by studying the influence of visual onsets that appear with the target stimulus. Such distractors can reliably modulate when a saccade is initiated, and these effects can be dissociated from the spatial modulation of where saccades land and the paths (or trajectories) of these movements. These behavioural effects depend on the spatial and temporal relationship between the saccade goal and competing distractor. Lévy-Schoen (1969) first reported an increase in saccade latency when two targets appeared simultaneously on opposite sides of fixation. We subsequently found that the latency increase, now known as the remote distractor effect (RDE), is maximal for near-simultaneous onsets and depends on distractor eccentricity, rather than the targetdistractor spatial separation as might be expected. In addition distractors can modulate the trajectories of saccades with deviation towards and away from the distractor being observed. The distractor-related modulation of saccade trajectories is coarsely coded and can be related to the timing of when a saccade is initiated - with deviation away from a distractor emerging slowly over time. Over the past few decades much progress has been made in developing our understanding of these deceptively simple behavioural effects. Here I will provide an overview of some of these findings and the mechanisms proposed to account for them based on converging behavioural and physiological findings.

\section{About the speaker}

Robin Walker graduated from The University of Newcastle upon Tyne and went on to study for a PhD at the University of Durham (UK) under the supervision of John M Findlay and Andy Young. After a post-doc spent at Durham he went on to a work with Chris Kennard in London. He then joined the Department of Psychology at Royal Holloway, University of London in 1997 where he is now a Professor of Cognitive Neuroscience.

His earlier work involved studies of oculomotor distractor effects in normal populations and in people with cortical brain damage along with interests in visual field defects (hemianopia and macular disease). His recent work includes projects examining the oculomotor functions of the human superior colliculus using functional brain imaging techniques (fMRI). Current projects also include studies of reading dynamic scrolling text in people with a loss of central vision as well as in non-visually impaired individuals. This work has led to the development of an iPad app as a reading aid for people with macular disease. 


\title{
Tuesday, August $18^{\text {th }}, 7.15 \mathrm{pm}-8.15 \mathrm{pm}$
}

\author{
Room: Audimax
}

\section{From the properties of the superior colliculus to saccades in the real world: Towards an integrated view of eye-movement control} Françoise Vitu

\author{
CNRS, Aix-Marseille Université, France \\ Francoise.Vitu-Thibault@univ-amu.fr
}

\begin{abstract}
At the end of the 19th century, Louis-Emile Javal discovered that our eyes make jerky, saccadic movements along the lines of text during reading. Since then, the study of saccadic behavior has grown exponentially and significant advances have been made in our understanding of the relationship between the successive shifts of our eyes over a text or a scene and ongoing visual and cognitive processes. Yet, whether and how the neurophysiology of the underlying oculomotor system, also studied for decades, does constrain eye guidance in real-world tasks has largely remained unexplored. Furthermore, despite converging evidence for the universality of several eye-movement patterns across tasks and stimuli, moving the eyes to a single point, reading a novel, viewing a landscape or searching for a face in the crowd are still considered as being fundamentally different from an oculomotor point of view. In my talk, I will present an alternative, integrated view of eye-movement control, that links well-known neural properties of the oculomotor system to eye guidance in a range of tasks and stimuli. Central to this view is the rather coarse and distorted representation of visual space in the superior colliculus (SC) where saccades are being programmed, and the related hypothesis that saccades are preceded by the activity of a population of SC neurons which is invariant to translation. I will show, based on a novel neuro-computational imaging approach of saccadic behavior, that the averaging of population activity in the distorted map of the SC strongly constrains the distribution of saccadic endpoints in simple saccadetargeting tasks, while providing the first behavioral evidence for population invariance in humans. I will then present several recent findings suggesting that the same mechanisms and constraints may also shape eye guidance during reading and scene viewing, and potentially account for universal eye-movement patterns (e.g. the Preferred Viewing Position effect). I will finally discuss how this population-averaging view can be reconciled with the more popular, cognitive account of eye-movement control.
\end{abstract}

\section{About the speaker}

Françoise Vitu studied Psychology at the University René Descartes in Paris, France, where she received her PhD in 1990, after working with J. Kevin O'Regan. She then went to the US for a post-doc with George W. McConkie at the University of Illinois at Urbana-Champaign and Albrecht Inhoff at the State University of New York at Binghamton. During the fall 1992, after another post-doc with Gery d'Ydewalle at the Catholic University of Leuven in Belgium, she was appointed as permanent researcher at the CNRS in Paris. In 1997, she was a NATO visiting researcher at the Beckman Institute (UIUC), and during the fall 2005, she was appointed as guest professor at the University of Potsdam, Germany. In 2004, she moved to Marseille, where she now is Director of Research (CNRS), and she heads with Eric Castet, the Perception and Attention group of the Laboratory of Cognitive Psychology at Aix-Marseille University. 


\section{Keynotes}

Françoise Vitu's early work focused on eye-movement control during reading, and aimed at determining the respective contributions of visuo-motor, linguistic, and attentional processes. It allowed her to estimate the spatial and temporal constraints associated with language-related influences, but also to isolate and characterize several oculomotor patterns that appear to be relatively universal. Being convinced that eye movements during reading and other perceptual tasks are primarily controlled by fundamental, visuo-motor principles, she recently extended her approach to object and natural-scene viewing, while also developing a new line of research that aims at linking the properties of saccades to the underlying neurophysiology of oculomotor and visual systems. 


\title{
Wednesday, August $19^{\text {th }}, 11.00$ am $-12.00 \mathrm{pm}$
}

\section{Room: Audimax}

\section{Fluctuations in attentional span during reading}

\author{
Jukka Hyönä \\ University of Turku, Finland \\ hyona@utu.fi
}

When reading a text, readers make a series of fixations from one word to the next so that the majority of words are fixated at least once. When fixating a word, readers visually attend to the fixated word in order to recognize it and comprehend its meaning in context. Readers also glean information from the word available in the parafovea around the fixated word. The area around the fixation point from which useful information for reading is extracted is here called the attentional span. It refers to the text area utilized from moment to moment in the ongoing text comprehension process. Attentional span can maximally cover the size of the perceptual span, but it is more limited when extra attentional resources are brought to bear. The main claim in my presentation is that attentional span fluctuates from moment to moment as a result of current processing demands and text and reader characteristics. I will present evidence in support of this claim. The key methods to examine fluctuations in attentional span are the moving window paradigm (McConkie \& Rayner, 1975) and the gaze-contingent display change paradigm (Rayner, 1975). In the moving window paradigm, a text window of chosen width moves along with the eyes so that the text within the window is intact but the text outside the window is in some way mutilated. Manipulating the size of text window and measuring when reading proceeds equally smoothly as in the no-window condition may be used to assess the size of the attentional span. In the display change paradigm, a critical area (typically a word) in the text is initially replaced with incorrect text information, which may or may not resemble in some way the correct word. The incorrect preview is only available in the parafovea. During the saccade launched to the critical word the preview is changed to its intended form. The extent to which readers attend to the parafoveally available preview is another way to assess the attentional span. In my presentation, I will go over how local processing demands (e.g., difficulty in word identification or in syntactic parsing), text features (e.g., spaced versus unspaced compound words), integrative processing at sentence level, global processing demands (e.g., reading goal and text difficulty), and individual differences (e.g., reading ability) influence the size of the attentional span. The overall message I wish to convey is that instead of debating whether word processing in reading is genuinely serial or parallel in nature, it is more fruitful to study the moment-to-moment fluctuations of the attentional span. Deeper understanding on the minute workings of visual attention during reading is likely to advance our understanding of the issue of serial versus parallel processing.

\section{About the speaker}

Jukka Hyönä received his PhD degree in psychology in 1993 from the University of Turku (Finland), where he now serves as a professor of psychology and as the Head of the Psychology division.

His main research focus is on the use of the eye-tracking method to study various visually based cognitive tasks, including, reading and text comprehension, multiple object tracking, 


\section{Keynotes}

attentional capture and recognition of peripherally presented stimuli. The emphasis is on capturing how processing of visual stimuli evolves over time. To date, his most significant scientific contributions have been made to the study of how the eyes (and visual attention) are guided through a written text. In that domain, his studies tap into different levels of written language comprehension - from word recognition via sentence parsing to comprehension of long expository texts. He has also applied the method to study attentional processes and eye guidance during reading. His research has been published in journals such as Journal of Memory and Language, Psychological Science, and Cognitive Psychology. He has published about 100 articles in peer-reviewed journals.

Together with Johanna Kaakinen, he has been one of the first to apply the eye-tracking method to investigate the processing of long expository texts as well as learning from authentic illustrated textbooks (in collaboration with Matti Hannus). Together with Manuel Calvo and Lauri Nummenmaa, he has successfully applied the method to examine whether peripherally presented emotional scenes capture the visual attention. Moreover, Oksama and Hyönä put forth a mathematically formulated model (MOMIT) of multiple identity tracking. At the moment, they are testing the seriality assumption of the model with eye movement registrations. 


\title{
Thursday, August $20^{\text {th }}, 11.00$ am $-12.00 \mathrm{pm}$
}

\author{
Room: Audimax
}

\section{Computational modeling of bottom-up and top-down visual attention in complex dynamic environments}

\author{
Laurent Itti \\ University of Southern California, United States of America \\ itti@usc.edu
}

Visual attention and eye movements in primates have been widely shown to be guided by a combination of stimulus-dependent or 'bottom-up' cues, as well as task-dependent or 'top-down' cues. Both the bottom-up and top-down aspects of attention and eye movements have been modeled computationally. Yet, is is not until recent work which I will describe that bottom-up models have been strictly put to the test, predicting significantly above chance the eye movement patterns, functional neuroimaging activation patterns, or most recently neural activity in the superior colliculus of human or monkey participants inspecting complex static or dynamic scenes. In recent developments, models that increasingly attempt to capture top-down aspects have been proposed. In one system which I will describe, neuromorphic algorithms of bottom-up visual attention are employed to predict, in a task-independent manner, which elements in a video scene might more strongly attract attention and gaze. These bottom-up predictions have more recently been combined with top-down predictions, which allowed the system to learn from examples (recorded eye movements and actions of humans engaged in 3D video games, including flight combat, driving, first-person, or running a hot-dog stand that serves hungry customers) how to prioritize particular locations of interest given the task. Pushing deeper into real-time, joint online analysis of video and eye movements using neuromorphic models, we have recently been able to predict future gaze locations and intentions of future actions when a player is engaged in a task. In a similar approach where computational models provide a normative gold standard against a particular individual's gaze behavior, machine learning systems have been demonstrated which can predict, from eye movement recordings during 15 minutes of watching TV, whether a person has ADHD or other neurological disorders. Together, these studies suggest that it is possible to build fully computational models that coarsely capture some aspects of both bottom-up and top-down visual attention.

\section{About the speaker}

Laurent Itti received his M.S. degree in Image Processing from the Ecole Nationale Superieure des Telecommunications (Paris, France) in 1994, and his Ph.D. in Computation and Neural Systems from Caltech (Pasadena, California) in 2000. He has since then been an Assistant, Associate, and now Full Professor of Computer Science, Psychology, and Neuroscience at the University of Southern California.

Dr. Itti's research interests are in biologically-inspired computational vision, in particular in the domains of visual attention, scene understanding, control of eye movements, and surprise. This basic research has technological applications to, among others, video compression, target detection, and robotics. Dr. Itti has co-authored over 150 publications in peer-reviewed journals, books and conferences, three patents, and several open-source neuromorphic vision software toolkits. 
Keynotes

\title{
Friday, August $21^{\text {st }}, 11.30$ am $-12.30 \mathrm{pm}$
}

\author{
Room: Audimax
}

The role of gaze control mechanisms in visual attention and visual perception

\section{Tirin Moore}

\author{
Stanford University, United States of America \\ tirin@stanford.edu
}

Recent work indicates that structures principally involved in movement planning and initiation also appear to influence sensory processing and underlie aspects of cognition. For example, neurons within the frontal eye field (FEF), an area of prefrontal cortex, play a key role in the programming and triggering of saccadic eye movements, but also influence signals within posterior visual cortex. This work not only implicates the FEF in the control of visual selective attention, but it demonstrates the profound influence that motor control circuitry can have on the processing within posterior sensory representations. I will describe recent evidence of this influence and discuss its relationship to perception and cognition.

\section{About the speaker}

Dr. Moore's laboratory at Stanford studies the primate visual system and how visual processing is integrated with executive control signals within prefrontal cortex, using the macaque monkey as a model species. His laboratory attempts to identify the neural circuits and neural computations necessary and sufficient to carry out fundamental perceptual and cognitive functions. Early on in his time at Stanford, his lab discovered that selective attention, a fundamental cognitive function, could be causally linked to the neural mechanisms controlling gaze.

Professor Moore received his Ph.D. from Princeton University in 1995, where he did his thesis work on the phenomenon of blindsight in monkeys with Charlie Gross. He was then a postdoctoral fellow at M.I.T. in the laboratory of Peter Schiller, where he studied the modulation of visual cortical signals during eye movements. He then moved back to Princeton as a research scientist where he began studying the neural mechanisms controlling visual selective attention. In 2003, he started his own laboratory at Stanford, where he is currently an Associate Professor of Neurobiology and Howard Hughes Medical Institute Investigator. Professor Moore's work is focused on identifying the neural circuits controlling visual perception and cognition, particularly visual attention. Professor Moore has been a Sloan fellow, a Pew Scholar, a McKnight Scholar, and was an Early Career Scientist of the Howard Hughes Medical Institute. In 2009, he received a Troland Award from the National Academy of Sciences for his work on visual attention. 


\section{Talks}


Talks

\section{Monday, August $17^{\text {th }}, 8.50$ am -10.30 am}

\section{Reading: Individual differences}

\section{Room: HS 7}

\section{Relative importance of text and reader characteristics for eye-movements in reading comprehension Victor Kuperman ${ }^{1}$, Kazunaga Matsuki ${ }^{1}$, Julie A. Van Dyke ${ }^{2}$ \\ ${ }^{1}$ McMaster University, Canada \\ 2 Haskins Laboratories, United States of America vickup@mcmaster.ca}

Both text and reader characteristics influence eye-movement patterns in reading for comprehension (Rayner, 2012). We present a new method that simultaneously estimates relative importance of multiple individual-differences measures and of benchmark text-level variables as predictors of reading behavior. Fifty-one proficient readers read passages from the Gray Oral Reading Test while their eye-movements were recorded; answered comprehension questions; and completed a large test battery assessing verbal, cognitive and executive skills.

Analyses using the Random Forest non-parametric regression method (Strobl et al., 2009) enabled a detailed comparative examination of: - groups of variables (e.g. individual variability mainly affects early eye-movement measures, while text properties affect later integrative processes), - specific variables (e.g., finger tapping performance is a strong predictor across the entire eye-movement record; Vocabulary Size and Rapid Automatized Naming are not); - the what and the where aspects of eye-movements (predictors of first fixation position and duration are in complementary distribution); and - interactions between lexical measures and individual skills (e.g. participant variability in skipping rate is mostly observed in long words; that in gaze duration shows in short ones).

This exploratory method complements a traditional focus on a small number of variables, and highlights venues for further confirmatory research. 


\title{
Do Chinese readers rely on phonological recoding when comprehending English texts?
}

\author{
Ying-Hua Guan ${ }^{1}$, Hsien-Yuan Hsu ${ }^{2}$ \\ ${ }^{1}$ National Taiwan Normal University, Taiwan, Republic of China \\ 2 Department of Leadership and Counselor Education, University of Mississippi, United States of \\ America \\ yhguan@ntnu.edu.tw
}

This study investigated if Taiwanese students relied on phonological recoding for comprehending English texts. Five reading conditions ("articulatory suppression", "reading aloud", "concurrent reading and listening to irrelevant speech", "concurrent reading and listening to the same text", and "silent reading") were used to examine whether reading comprehension would be hindered if the processes of phonological recoding were interfered. We observed subjects' eye movements during reading and compared their outcomes in reading comprehension. Moreover, we also examined whether text complexity serves as a mediate factor contributing to subjects' reliance on phonological recoding in the processes of English reading-comprehension. The results showed that reading conditions had no significant effects on subjects' performance on reading comprehension, but exerted significant effects on subjects' eye movements during reading. Text complexity did not affect subjects' dependency on phonological recoding. It seems that our subjects did not rely much on phonological recoding when comprehending English texts of different complexities and were able to access meaning via the orthographic-semantic route when the processes of phonological recoding were interfered. The results of word-based eye-movement analysis suggest that phonological recoding might still participate in the word-recognition processes, even though it did not had significant effect on subjects' performance on reading comprehension.

\section{Eye movement control in oral vs. silent reading: Evidence from a large sample of very young readers. \\ ${ }^{1}$ Universität Wuppertal, Germany \\ 2 Florida Center for Reading Research, United States of America vorstius@uni-wuppertal.de}

Even though reading aloud is one of the dominating cognitive tasks children are asked to perform, little is known about eye movement control in this task. We studied the development of oral and silent reading fluency and their relations with comprehension. Oculomotor data represent results from two assessment periods (T1 and T2) in year one of a large-scale longitudinal study $(\mathrm{N}=400)$. First graders read sets of single line sentences taken from a standardized reading test (TOSREC).

Results show a large increase in reading speed from T1 to T2, with students lower in T1 fluency scores showing greater gain. All three components of word viewing time, initial fixation duration, refixation duration and re-reading time are inflated in oral reading. Critically, this differences is larger for students with lower T1 fluency scores and most pronounced in later measures, especially re-reading time.

Two sets of factors can be made responsible for this pattern of results. First, there may be some influence of pronunciation difficulty and less systematic eye-voice coordination, leading to frequent catch-up saccades. Second, suggested by local fixation patterns, inflated re-reading times appears to indicate that in oral reading more mental effort is required to achieve an acceptable level of comprehension. 


\title{
Individual differences in language skills affect children's eye movement characteristics
}

\author{
Lyuba Mancheva ${ }^{1,2,3}$, Benoit Lemaire ${ }^{2}$, Anne Guérin-Dugué ${ }^{1}$, Jean Ecalle ${ }^{3}$, Sylviane \\ Valdois $^{2}$ \\ ${ }^{1}$ GIPSA-lab, University of Grenoble Alpes, Grenoble, France \\ 2 CNRS, LPNC UMR5105, F-38000 Grenoble, France \\ ${ }^{3}$ Laboratoire d'Etude des Mécanismes Cognitifs, Université de Lyon 2, France \\ Imancheva@yahoo.fr
}

At the level of eye movement control, what distinguishes poor from good comprehenders, poor from good word identifiers and poor from good grapheme-phoneme mappers? Our goal was to identify how differences in language skills modulate children's eye-movements. The eye movements of 75 children were analyzed while they read a 212 word text. Their orthographic, phonological and comprehension skills were further assessed. For each of these skills, participants were divided into three groups: poor, average and good. Results show that good orthographiers performed longer saccades, skipped more words and performed one fixation per word more often than poor orthographiers. Actually, they needed fewer fixations to read the entire text.

Good grapheme-phoneme mappers showed shorter gaze duration, shorter total reading time and fewer refixations than poor grapheme-phoneme mappers. Lastly, good comprehenders performed longer saccades, made fewer refixations, and their total reading time on words was shorter than for poor comprehenders.

These results suggest strong significant relationships between children language skills and the way their eyes go through a text. We discuss the implications of our findings for helping children with reading difficulties.

\section{Age of Acquisition Effects in Arabic Reading}

\author{
Sana Bouamama, Simon P. Liversedge, Ehab W. Hermena, Denis Drieghe \\ University of Southampton, United Kingdom \\ S.Bouamama@soton.ac.uk
}

The Age of Acquisition (AoA) effect on word recognition processes has been found in multiple visual word recognition tasks and during reading where an early acquired word receives shorter fixation durations than a late acquired word (Juhasz \& Rayner, 2006). We aimed to investigate the AoA effect in Arabic but were confronted with the lack of a suitable database for this world language. After an extensive survey of teaching instruction methods across the Arabic world, we established that instruction at specific ages was very tightly linked with specific chapters of the Quran and this was consistent across countries. A stimulus set was created by selecting words based on their first appearance in early versus late taught chapters of the Quran, and matched on word length and frequency. We also collected subjective AoA norms (whereby participants not taking part in the experiment estimate the AoA), which supported the proposed difference in AoA between the two groups of stimuli. Using this novel method of stimuli selection, a subsequent eye-tracking experiment in which native speakers read the target words embedded in sentences, showed for the first time the AoA effect in Arabic through shorter fixation durations on early compared to late acquired words. 


\title{
Integrating eye movements in research on expertise and skill acquisition
}

Room: HS 30

\section{Effects of Professional Visual Search Experience on Domain-General Visual Search Tasks}

\author{
Laura E. Matzen, Michael J. Haass, Laura A. McNamara, Susan M. Stevens-Adams, \\ Stephanie N. McMichael \\ Sandia National Laboratories, United States of America \\ lematze@sandia.gov
}

Given the importance of visual search in high-consequence decisions, such as medical diagnoses and security screening, there is a great deal of interest in understanding how professionals develop expertise in visual search and how that expertise influences their performance. In the present study, we compared two groups of professional visual searchers and novices on a battery of domain-general and domain-specific tasks. The domain-general tasks tested mental rotation ability, spatial working memory capacity, useful field of view, parallel and serial visual search, and visual inspection. The first group of professionals consisted of Synthetic Aperture Radar (SAR) imagery analysts who search for information in radar images. The second group consisted of engineers who view time series data to find and characterize anomalous tests. We found that both groups of professionals outperformed novices on visual search tasks within their domain of expertise. On the domaingeneral visual search tasks, the SAR analysts were significantly faster than novices while the engineers' performance did not differ from that of the novices. These results indicate that professional experience with visual search can influence domain-general visual search performance, but that these effects are dependent on the nature of the experience.

\section{Saliency Estimation for Advanced Imaging Scenes Using Pixel Statistics}

\author{
Michael J. Haass, Laura E. Matzen, Laura A. McNamara, Kristina R. Czuchlewski \\ Sandia National Laboratories, United States of America \\ mjhaass@sandia.gov
}

Many computational models have been developed to estimate visual saliency for naturalscene images and have been validated using eye tracking fixation maps. However, it is difficult to find examples of saliency models applicable to the less-natural scene properties produced by advanced imaging sensors such as synthetic aperture radar (SAR) or multi-energy $\mathrm{x}$-ray. Because these advanced sensors are designed to produce data for interpretation by human analysts, traditional saliency models will have some applicability - information is still encoded and displayed using standard visualization parameters such as contrast and color. However, the very nature of these advanced imaging technologies distorts certain attributes of the scene. For example, SAR imagery contains oddities in shadowing and spatial relationships. In these cases, pixel-statistical methods can be used to segment the scene and characterize the segment properties. These properties can serve as filters to modulate traditional saliency estimates. In a study of experienced SAR imagery analysts, we show this approach produces saliency estimates with up to four times better agreement with eye tracking fixation maps. Using eye tracking technology to explore relationships between traditional saliency models and pixel-statistical properties we can understand eye movements of domain experts interacting with imagery from today's most advanced sensors. 


\title{
Challenges in Eyetracking Data Analysis: From the Laboratory to the Wild World of Information
}

\author{
Laura A. McNamara, David Stracuzzi, Kristina R. Czuchlewski \\ Sandia National Laboratories, United States of America \\ lamcnam@sandia.gov
}

Human-information interaction researchers rely on user interaction logs to study cue-driven behaviors associated with information foraging. Gaze data could provide valuable complementary data for understanding the perceptual and cognitive processes that attend information foraging in online information environments. However, eye tracking technologies evolved in a research paradigm that privileges highly constrained tasks with static stimuli to test hypotheses about perception and attention. In particular, the design, data collection and analysis software tools do not translate easily to the complicated, user-driven workflows that characterize information foraging "in the wild."

New algorithms and software for gaze tracking studies could expand the applicability of eye tracking systems for the complex perceptual cognitive workflows associated with information foraging. In particular, we suggest that gaze data and the associated user behaviors and stimuli can be treated as a high-dimensional point dataset amenable to a number of decomposition and pattern analysis techniques, including matrix decomposition and graph algorithms. Expanding the repertoire of mathematical models, algorithms and software used with eye tracking systems could provide an entirely novel source of data for documenting the search behaviors that characterize information foraging in large, complex data environments.

\section{Air traffic control: Visual expertise in a dynamic problem solving task Halszka Jarodzka $^{1,2}$, Paula Gouw ${ }^{1}$, Ludo Van Meeuwen ${ }^{1}$, Saskia Brand-Gruwel ${ }^{1}$ \\ ${ }^{1}$ Open University of the Netherlands, Netherlands \\ ${ }^{2}$ Humanities Lab, Lund University, Sweden Halszka.Jarodzka@ou.nl}

Many professions require the examination of complex, dynamic images (e.g., medical specialists, air-traffic controllers). Thus, in the training of these professions, students have to learn to visually examine and interpret such images. To construct educational material and strategies for this training, it is important to understand how visual expertise develops in such professions. In the current study we investigated the domain of air traffic control. Twelve air traffic controllers of three expertise levels (experts, intermediates, and beginners) worked for ten minutes on a dynamic air traffic control simulator while their eye movements were recorded. Results show that experts exceed the other groups on task performance. On a perceptual level, however, only beginners and intermediates differed significantly: beginners mainly focus on the radar area they were responsible for. Intermediates, in contrast, looked longer on neighboring areas from which airplanes were coming in or leaving to. These findings indicate (1) how beginners should re-direct their attention to other areas to be able to predict upcoming situations and (2) that experts do not look differently at the images than the other groups, but process the information differently so that they end up with better solutions. 


\section{The Influence of Culture and Expertise on Teacher Gaze Patterns Nora Ann Mclntyre, Robert M. Klassen \\ Psychology in Education Research Centre, Department of Education, University of York, United Kingdom \\ nora.mcintyre@york.ac.uk}

That experts distribute their visual attention more effectively than novices is well documented for a number of professions. Cultural differences are also generally expected in the social signals conveyed by eye contact. The present study explores two factors within the educational context by making expert-novice and east-west comparisons of teacher gaze patterns. Data was collected from 40 secondary school teachers: 20 from the UK; 20 from Hong Kong. Eye-tracking glasses were used to collect gaze and verbal data simultaneously during teacher-centred learning. Classroom climate questionnaires were then administered to explore whether teachers' gaze patterns correlated with their interpersonal profiles. Retrospective thinkaloud was then carried out with each participant for specific perceptual and communicative intents. Simultaneous and retrospective verbal data were used for interpreting teachers' cognitive processes. Preliminary analysis suggests that (1) experts and novices display contrasting gaze patterns. (2) Eastern experts contrast with western experts in their gaze patterns for classroom perception and communication. (3) Cultural and expertise differences have emerged in teacher gaze patterns and correlating teacher interpersonal profiles. We will be discussing options of analysis techniques for real-world eye-tracking. One educational application for this is in the related aspects of teacher training curricula. 


\title{
Talks
}

\section{Eye behavior: Perceptual effects and cognitive influences}

Room: HS 31

\section{The speed-up of perceptual time during blinks}

\author{
Mark Wexler, Marianne Duyck, Thérèse Collins \\ CNRS/Université Paris Descartes, France \\ mark.wexler@parisdescartes.fr
}

\begin{abstract}
Why do we hardly perceive the sensory consequences of eye blinks? Blinks create nearcomplete blackouts lasting about $100 \mathrm{~ms}$, a substantial time interval for vision. Blackouts that simulate blinks are much more salient and seem to last much longer than actual blinks. Visual stability during saccades has received a great deal of attention, but stability across blinks has been unjustly neglected. Past work on visual stability during blinks has focused on detection, notably finding that visual thresholds are higher during blinks; however, higher thresholds cannot explain how we almost completely miss the blackout, whose luminance decrement is surely above any threshold. We propose an alternative mechanism: blinks seem so brief and inconsequential because perceptual time speeds up during the blink. We tested this hypothesis by artificially prolonging the blackout: we extinguished the room lights when the blink began, turning them back on after a random delay following the end of the blink. Subjects reported the total perceived duration of the blackout. We found that time intervals during blinks are perceived at about $50-60 \%$ of their duration as compared to intervals immediately following the blink. This effect is visual, being absent for comparable judgments of the duration of auditory tones.
\end{abstract}

\section{A common spatial map for Auditory and Visual Targets revealed by Landmark-induced positional shifts}

\author{
David Aagten-Murphy, Martin Szinte, Heiner Deubel \\ Ludwig-Maximilians-Universität München, Munich, Germany \\ David.aagtenmurphy@gmail.com
}

The visual stimuli present before and after saccades have been shown to act as landmarks for visual target localisation. Here we extended these findings to the investigation of auditory stimuli and found that auditory localisation was altered by eye-movements, despite the head and ears remaining stationary. Furthermore, visual targets were found to act as landmarks for the localisation of sounds across saccades. Two brief visual (LED) or auditory (white noise) probes were presented at different locations before and after a saccade. By varying the pre- and post-saccadic probe locations, and the saccade target (our landmark), we evaluated changes in visual and auditory localisation performance. Consistent with previous findings, we found a shift in subject's localisation of visual stimuli proportionate to the landmark's displacement. Critically, despite stationary head and ears, landmark displacements induced half-proportional shifts in auditory stimuli localisation. These results indicate that visual landmarks, as with visual stimuli, were used to spatially align the pre- and post-saccadic auditory stimuli for localisation judgments. That visual landmark stability mechanisms can so strongly act on auditory events suggests that a common, supra-modal spatial map is utilized to maintain a stable representation of objects of both modalities. 


\title{
Unseen transsaccadic changes induce re-calibration of perceived peripheral size
}

\author{
Matteo Valsecchi, Karl R. Gegenfurtner \\ Justus-Liebig-University Giessen, Germany \\ matteo.valsecchi@psychol.uni-giessen.de
}

The size and shape of objects do not appear to change as we move our eyes despite the massive distortion in the cortical projection of the visual field known as cortical magnification. We test experimentally whether a mechanism discounting predictable visual changes associated with saccades effectively calibrates central and peripheral size perception.

In each of the 500 trials, observers first compared the relative size of a central and a peripheral $\left(20^{\circ}\right)$ stimulus. Subsequently, they looked at the peripheral stimulus and performed a difficult discrimination of its shape. Size PSEs were obtained in a sliding window of 100 trials.

After the first 100 trials, we introduced a transsaccadic change (up to $\pm 10 \%$ ) in the size of the saccade target. By the end of the session, the perceived size in the observer group exposed to transsaccadic size decrease was $7.3 \%$ lower compared to the group exposed to transsaccadic size increase.

The perceptual recalibration established transsaccadically was still expressed when observers ceased to execute saccades, it endured on the next day and it extended to untrained retinal locations of equal eccentricity.

We suggest that the calibration of peripheral size appearance is established and continuously fine tuned based on trans-saccadic prediction error.

\section{Low-level versus high-level effects on saccadic landing positions on daily-life objects}

\author{
Lotje van der Linden, Sebastiaan Mathôt, Françoise Vitu \\ Aix-Marseille Université, LPC UMR 7290, Marseille, France \\ I.vanderlinden@cogsci.nl
}

We investigated eye guidance by contrasting the time course of low-level saccadeprogramming mechanisms with the time course of higher-level object-related effects. We presented participants with (1) isolated photographs of action-related objects whose actionperforming side pointed to the left or to the right, and (2) meaningless non-objects of which the low-level properties (texture, shape, center of gravity [CoG]) were matched to the real objects. We measured where participants' eyes landed relative to the CoG of the peripheral stimuli as a function of stimulus type (objects versus non-objects) and handle orientation. Based on our previous findings, we predicted that the contribution of low-level visuomotor mechanisms would dissipate over time, thereby making room for higher-level, object-based effects. In line with this, we found that landing positions of early-triggered saccades towards non-objects were narrowly distributed around the CoG. When saccade latencies increased, this distribution became wider, though remaining centered near the CoG. Thus, there was no systematic bias towards either side of non-objects. Saccades towards real objects, on the other hand, initially landed towards the CoG but showed a gradually increasing bias towards the objects' action-performing side when latencies increased. This suggests that higher-level processes build up over time and override low-level visuomotor mechanisms. 
Talks

\title{
In search of oculomotor capture during film viewing: implications for the balance of top-down and bottom-up control in the saccadic system
}

\author{
Stephen John Hinde ${ }^{1}$, Tim J. Smith ${ }^{2}$, lain D. Gilchrist ${ }^{1}$ \\ ${ }^{1}$ University of Bristol, United Kingdom, United Kingdom \\ 2 Birkbeck, University of London, United Kingdom \\ stephen.hinde.06@bristol.ac.uk
}

In the laboratory, the abrupt onset of a visual distractor can generate an involuntary orienting response: this oculomotor capture effect has been reported in a large number of studies (e.g. Theeuwes et al. 1998; Ludwig and Gilchrist 2002). However the visual stimuli used in these experiments have tended to be static and had none of the complexity, and dynamism of more natural visual environments. In addition, the primary task for the participants has tended to be both boring and repetitive. Both of these factors may have lead to an overestimation of the extent to which capture occurs and the importance of this phenomena in everyday visual behaviour. To address this issue, in the current series of studies we presented abrupt and highly salient visual distractors away from fixation while participants watched a film. No evidence of spatial capture of gaze was found. However, evidence of distraction was found in the form of increased duration of the ongoing fixation in a manner analogous to a remote distractor effect (Walker et al. 1997). These results suggest that during dynamic scene perception the oculomotor system may be under far more top-down or task based control than traditional laboratory based tasks have previously suggested. 


\title{
Eye movements: Smooth pursuit, manual tracking, control
}

\section{Room: HS 32}

\author{
Properties of human smooth pursuit adaptation \\ Jos N. van der Geest ${ }^{1}$, Berna M. Isik ${ }^{1}$, Maarten A. Frens ${ }^{1,2}$, Giulietta S. Monasso ${ }^{1}$ \\ ${ }^{1}$ Neuroscience, Erasmus MC Rotterdam, Netherlands \\ ${ }^{2}$ Erasmus University College, Rotterdam, Netherlands \\ j.vandergeest@erasmusmc.nl
}

Environmental changes can induce short-term adaptation of both reflexive and voluntary eye movements. In a set of experiments we investigated the malleability of human smooth pursuit eye movements.

Participants looked at a target that moved away from a central stationary position with a fixed velocity. Subjects made a saccade in order to catch up with the already moving target, followed by smooth pursuit of the target. In baseline trials, the velocity of the preand post-saccadic target was the same and the velocity of the post-saccadic smooth pursuit matched the pre-saccadic target velocity nicely. In adaptation trials, however, we could instantaneously change the velocity of the target during the catch-up saccade.

We looked at persistent changes in post-saccadic smooth pursuit behaviour after a series of adaptation trials. We observed that 50 adaptation trials was sufficient to change postsaccadic smooth pursuit velocities over several trials, in response to consistent decreases and increases in target speed. We also observed that changes in direction or even direction reversal induces persistent adaptation. Participants were also able to ignore distractors that appeared during the catch-up saccade and moved with a different velocity.

\section{Concurrent manual tracking enhances pursuit eye movements}

Diederick C. Niehorster ${ }^{1,2}$, Wilfred W.F. Siu ${ }^{1}$, Li Li ${ }^{1}$

${ }^{1}$ Department of Psychology, The University of Hong Kong, Hong Kong Special Administrative Region, People's Republic of China

2 The Humanities Laboratory, Lund University, Sweden dcnieho@gmail.com

It is known that concurrent manual tracking enhances smooth pursuit of a predictable moving target. Here we examined such facilitation when the target moved unpredictable.

In the eye-hand condition, participants were asked to follow a cyan Gaussian target $(\operatorname{sigma}=0.6)$ whose horizontal position was perturbed by the sum of seven harmonicallyunrelated sinusoids $(0.1-2.19 \mathrm{~Hz})$. Simultaneously, they used a mouse to keep the horizontal position of a red Gaussian cursor ( $8^{\circ}$ below) aligned with the target. In the eye-alone condition, the target and cursor motion recorded in the eye-hand condition were replayed, while participants tracked the target only with their eyes.

Across 15 participants, baseline RMS eye tracking position error did not differ between the two conditions. However, frequency-response analysis of smooth pursuit eye movement with saccades removed showed that pursuit gain was significantly higher for the eye-hand than the eye alone condition (Cohen's $d=0.62$ ). Consequently, significantly fewer saccade were made in the eye-hand (mean \pm SE: $215 \pm 6$ per 90 -s trial) than in the eye-alone condition $(250 \pm 6)$ while catch-up saccade amplitudes and durations did not differ.

We conclude that concurrent manual tracking enhances smooth pursuit, and that this effect is not specific to predictable target movement but is a fundamental property of eye-hand coordination. 
Talks

\title{
The Biometric Identification Based on Eye and Mouse Movement Traits
}

\author{
Katarzyna Harezlak, Pawel Kasprowski \\ Silesian University of Technology, Poland \\ katarzyna.harezlak@polsl.pl
}

The aim of the research was to provide a new approach to the biometric identification using the combined features analysis of eye and mouse movement signals. The contribution of the studies is the first attempt to build the identification model based on fusion of these two different biometric traits. There were appropriate experiments organized, in which 45 participants took part.

The participants' task was to click four of the ten digits displayed on a screen. It was assumed that he/she was looking at a digit while clicking on it. Both mouse and eye gaze positions were recorded during this trial. Characteristic features called signals were extracted from both type of gathered data. Gaze and mouse position coordinates, velocities and accelerations can be taken as signal examples. Each signal was converted into features forming samples used in a classification process. There were three samples' types taken into account: - statistic values obtained from $\max , \mathrm{min}$, avg and std aggregating functions, - histograms of signal values, - similarity matrix - a matrix representing distance between trials.

As a classification method SVM classifier was applied. The results obtained are promising, confirmed the rightness of the studies undertaken.

\section{Physical causality guides eye movements}

\section{Marcus Missal, Kim Wende}

Université catholique de Louvain, Belgium

marcus.missal@uclouvain.be

Causality is a unique feature of human perception. Causality is a perceptual prior that allows us to interpret and predict upcoming events like the outcome of the collision between objects. We present here a behavioral investigation of the influence of physical causality during visual pursuit of object collisions.

Pursuit and saccadic eye movements of human subjects were recorded during ocular pursuit of two concurrently launched targets, one that moved according to the laws of Newtonian mechanics (the causal target) and the other one that moved in a physically implausible direction (the non-causal target). Eye movements were used as behavioral measures to estimate the influence of physical causality on sensorimotor processing.

We found that anticipation of collision evoked early smooth pursuit decelerations. This anticipatory deceleration was not present in a control condition where there was no collision. Moreover, saccades to non-causal targets were hypermetric and had latencies longer than saccades to causal targets.

We conclude that pursuit of a target that followed a non-causal trajectory evoked a prediction error as indicated by increased saccadic latencies. The human visual system is rapidly and implicitly distinguishing causal from non-causal motions. Therefore, physical causality could be represented early in sensori-motor systems. 


\section{OpenEyeSim: a biomechanical model for studying oculomotor control Alexander Priamikov ${ }^{1}$, Vikram Narayan ${ }^{1}$, Bertram Shi ${ }^{2}$, Jochen Triesch ${ }^{1}$ \\ ${ }^{1}$ Frankfurt Institute for Advanced Studies, Germany \\ ${ }^{2}$ Hong Kong University of Science and Technology priamikov@fias.uni-frankfurt.de}

In this work we introduce OpenEyeSim, a novel 3-dimensional biomechanical simulation of the oculomotor plant (OMP). The OMP model comprises six extra-ocular muscles (EOMs) per eye. Virtual cameras sense the images provided by the two eyes, allowing for closedloop control of eye movements. To this end, the model is embedded in a 3-d simulated computer graphics environment.

The oculomotor plant (OMP) is designed on the basis of the current state-of-the-art vis-a-vis the non-linear dynamics of the EOMs. The proposed OMP also factors in the elasticity of eye-surrounding tissues and muscle pulley dynamics. Given EOM innervations, our model generates realistic gaze trajectories with appropriate OMP dynamics matching empirical observations. Also, our model produces appropriate muscle forces for different static positions corresponding to empirical data. It thus facilitates the understanding of neural control and development of the various eye movements. It also serves for testing different hypotheses pertaining to sensory coding and development of receptive fields in an active vision context. As an initial step, we have exploited the OpenEyeSim platform to model the joint development of disparity tuning and vergence control during infancy. In conclusion, we introduce OpenEyeSim as a versatile tool for developing sophisticated models of human oculomotor control. 


\title{
Monday, August $17^{\text {th }}, 1.20 \mathrm{pm}-3.00 \mathrm{pm}$
}

\section{Reading: General I}

\author{
Room: HS 7
}

\section{A Joint Analysis of Fixation Locations and Fixation Durations during Reading \\ Reinhold Kliegl \\ University of Potsdam, Germany \\ kliegl@uni-potsdam.de}

Reading research often uses fixation duration (FD) or fixation location (FL) as dependent variables to check effects of covariates (e.g., length, frequency, predictability of fixated and neighboring words). Moreover, FL also serves as a covariate of FD (e.g., inverted optimal viewing position [IOVP] effect). However, the independent analyses of FD and FL fail to capture the redundancy between these measures and their relative sensitivity to covariates. Linked Linear Mixed Models (LLMMs) are proposed for joint analysis of FL and FD in their sequential dependency and the correlation of means and covariate effects due to subject- or item-related clustering. LLMMs consist of two linear mixed models (LMMs), one for FL and one for FD, both as function of covariates. The second LMM also includes the outcome of the first LMM (i.e., the predicted and residual FL) as covariates. The analysis allows a distinction between direct effects and indirect effects (i.e., mediated through fixation location) of word properties on fixation durations. The method is illustrated with analyses of eye movements from reading Chinese and German sentences. Results confirm the robustness of distributed processing during the perceptual span and that the IOVP effect is primarily due to saccadic errors.

\section{Eye Movements and the Effect of Word Frequency on Display Change Detection during Reading}

\author{
Bernhard Angele ${ }^{1}$, Timothy J. Slattery ${ }^{2}$, Keith Rayner ${ }^{3}$ \\ ${ }^{1}$ Bournemouth University, United Kingdom \\ 2 University of South Alabama, United States of America \\ 3 University of California San Diego, United States of America \\ bangele@bournemouth.ac.uk
}

We used a display change detection paradigm (Slattery, Angele, \& Rayner, 2009) to investigate whether display change detection uses abstract letter codes and whether display change detection is affected by the processing difficulty of the word preceding the boundary which triggers the display change. We report two main findings: first, subjects were significantly more sensitive to display changes when the change was from a non-wordlike preview than when the change was from a wordlike preview. Additionally, eye movement measures showed a significant orthographic parafoveal-on-foveal effect on the pre-boundary word of the non-wordlike preview compared to the wordlike and the identical preview conditions. On the other hand, the preview benefit effect on the target word was not affected by whether the preview was wordlike or non-wordlike. Second, we did not find any influence of preboundary word frequency on display change detection performance. Our results suggest that display change detection does not use the same cognitive mechanisms that are involved in parafoveal preprocessing during natural reading. Instead, we propose that parafoveal processing takes place in two stages: an early, orthography-based, pre-attentional stage, and a late, attention-dependent lexical access stage. 


\title{
Word processing during sentence reading: Eye movement behaviour of young and older adult readers
}

\author{
Victoria A. McGowan, Kayleigh L. Warrington, Sarah J. White, Kevin B. Paterson \\ University of Leicester, United Kingdom \\ vm88@le.ac.uk
}

Previous research indicates that older adults (aged 65+) experience particular difficulties in reading compared to young adults (aged 18-30). However, the origins of these age-related changes are poorly understood. Accordingly, two eye tracking experiments are presented that examine whether young and older adult readers differ in two important components of early stages of word processing during reading. Experiment 1 examined adult age differences in the flexibility of letter position coding by presenting words in sentences normally (e.g. problem) or transposing letters at a word's beginning (rpoblem), interior (porblem), or end (probelm). The effects of these transpositions on eye movement behaviour were similar for young and older adults, suggesting similarly flexible encoding of letter positions during reading. Experiment 2 examined effects of stimulus quality by manipulating the visual contrast of text. The results indicate that older adults had greater difficulty reading low-contrast text than young adults. Both experiments also included a critical target word manipulation of word frequency, providing tests of whether the effects of letter position coding and stimulus quality are modulated by lexical processing. Together the results of these experiments have important theoretical implications for the nature of older adults' word recognition processes during reading.

\section{The effect of capitalization on reading in German \\ Dennis Pauly, Guido Nottbusch \\ University of Potsdam, Germany \\ dpauly@uni-potsdam.de}

German orthography systematically marks nouns by capitalization. Our theory is that the reader benefits by the uppercase-letter receiving syntactic and semantic information, which makes the processing of sentences easier. We designed an experiment (54 participants) in which the function of German capitalization rules should become obvious in the eyemovements. The task was to read single sentences presented half following German standard orthography (CAP), half in lowercase-spelling (NoCAP). The sentences were (by using semantic priming) systematically manipulated on a target word $(\mathrm{N})$ in order to build up a strong expectation of a noun, which finally got counteracted (experimental condition; EXP) $-\mathrm{N}$ was an attribute for the following noun $(\mathrm{N}+1)$ instead. In an additional control condition $(\mathrm{CON})$, in which it was crucial that the interpretation of a noun was extremely unlikely, $\mathrm{N}$ was replaced by a word of the same length and similar frequency.

Analogue example (English): The sick w/Writer had a cold(EXP;N)/blue(CON;N) N/nose $(\mathrm{N}+1)[\ldots]$.

Significant effects on N/N+1 are detectable in Gaze and Total Fixation Duration dependent on the spelling CAP/NoCAP and in Total dependent on EXP/CON. Further analyses and interpretations regarding the German capitalization system will be reported in my presentation. 
Talks

\title{
An Eye-Movement Study of Comprehension-Based Silent Reading Efficiency Across Grade Levels in U.S. Students
}

\author{
Alexandra N. Spichtig, Jeffrey Pascoe, John Ferrara, Angela Zinno, Elias Tousley \\ Reading Plus, United States of America \\ alex@readingplus.com
}

Eye-movement recordings were obtained while 2,258 students in even grades (2-12) read grade-leveled texts with demonstrated comprehension. Recordings were compared across grades and reading rate quartiles to characterize differences in fixations per word, fixation durations, and regressions per word.

Comparisons revealed that students in higher reading rate quartiles made fewer fixations per word, had shorter fixation durations, and made fewer regressions per word. The same pattern of increasing efficiency characterized grade advancement, but pairwise comparisons revealed exceptions such as: (a) comparisons between grades 6 and 8, where fixations and regressions increased while fixation durations and reading rates stagnated; (b) students in the lowest quartile showed little growth in efficiency beyond grade 4; and (c) students in the second lowest quartile showed little growth beyond grade 6 .

Overall, the results suggested that students in the lower quartiles were persisting in the sub-lexical analysis of text; requiring multiple fixations per word, and more often finding it necessary to go back and re-fixate on words. Among students in grades 10 and 12, only those in the highest reading rate quartile had fixation rates of one fixation per word or less, suggesting a degree of automaticity comparable to skilled adult readers. 


\title{
Eye movements and reading: Expertise
}

\author{
Room: HS 30
}

\section{The perceptual component of chess expertise: Evidence from eye movements}

\author{
Eyal M. Reingold ${ }^{1}$, Heather Sheridan ${ }^{2}$ \\ 1 University of Toronto, Canada \\ 2 University of Southampton, United Kingdom \\ reingold@psych.utoronto.ca
}

The remarkably efficient performance of chess experts reflects extensive practice with domain-related visual configurations (for a review, see Reingold \& Sheridan, 2011). To study the perceptual component of chess expertise, we monitored the eye movements of expert and novice chess players while they engaged in a wide range of chess-related tasks. Experiment 1 demonstrated that the eye movements of experts (but not novices) rapidly differentiated between regions of a chessboard that were relevant versus irrelevant to the best move on the board. Similar to Experiment 1, Experiment 2 showed that chess experts (but not novices) rapidly identified complex chess-related visual patterns during a challenging chess-related visual search task. Finally, Experiment 3 showed that experts were better than novices at disengaging their attention from familiar (but suboptimal) chess solutions, when a better solution was available in a different region of the board (for a similar paradigm, see also Bilalić, McLeod, \& Gobet, 2008). Taken together, these experiments highlight the perceptual component of visual expertise, while also demonstrating the flexibility of experts in adapting to a wide range of challenging experimental paradigms. We discuss the implications of our findings for models of eye movement control and domain-general theories of visual expertise.

\section{The perception of the number line by adults and preschoolers: eye-movements during the teaching process.}

\author{
Anna Shvarts, Anatoly Krichevets \\ Lomonosov Moscow State University, Russian Federation \\ shvarts.anna@gmail.com
}

The eye-tracking methodology is widely used in novices-experts researches; it discloses the difference between educated and naïve perception. In our research we explored the process of transmission of perceptual strategies from experts to novices, namely the teaching of counting on the number line from 0 to 10 . We invited 6 couples of a child and a parent and we analyzed the adults' strategies of counting, the strategies chosen by adults for teaching, and children's strategies after they were taught. In each case 10 tasks were presented requesting the detection of a particular point on the number line. In all three cases the strategies were essentially different $(\chi 2(14)=26.397, \mathrm{p}=0.023)$.

Our main focus was on the qualitative analysis of communication between a child and his/her parent during teaching. We analyzed the synchronized video of adults' pointing gestures, audio of parents' and children's speech and children's eye-movements. The high rhythmical synchronization of gestures, salient verbal expressions and eye fixations was shown. The main conclusion is that a learner does not merely follow her parent's directions; she rather anticipates them and constitutes the meaning of her parent's gestures and words in accordance with the initial task.

Supported by RFBR, grant \#15-06-06319 
Talks

\title{
Eye movements during visual search of infrared images
}

\author{
Mackenzie G. Glaholt, Grace Sim, Philips Laou \\ Defence Research and Development Canada, Toronto, Canada \\ Mackenzie.Glaholt@drdc-rddc.gc.ca
}

Infrared imaging is widely used by law-enforcement and military to enhance visual search performance. Presently we provide an empirical basis for these enhancements and account for them in terms of the temporal dynamics of eye movements and visual attention. Eye movements were recorded while subjects searched for and identified human targets in forested scenes that were presented on a computer monitor. These real-life scenes were photographed by cameras that captured information in the short-wave infrared (SWIR, 0.9$1.7 \mu \mathrm{m}$ ), long-wave infrared (LWIR, 8-12 $\mu \mathrm{m}$ ), or visible (VIS, 400-700 $\mathrm{nm}$ ) spectrum. Visual search trials were segmented into a 'detection' interval prior to the first target fixation and an 'identification' interval following the target fixation and prior to the identification response. Consistent with our hypothesis we found that LWIR images produced the shortest detection intervals. However, LWIR also produced the longest identification intervals and the lowest response accuracy. In contrast, SWIR produced the shortest identification intervals and the highest response accuracy. VIS images produced the longest detection intervals and intermediate identification intervals and response accuracy. We conducted stimulus analysis and were able to explain this pattern of behavioural findings in terms of the luminance contrast, saliency, and spatial frequency characteristics of the images.

\section{Sight Reading Expertise, Eye Movements and What Happens when you Remove the Bar Lines.}

Patricia Arthur

University of New South Wales, Australia

patricia.arthur@student.unsw.edu.au

Recent research has found that performance at a defined level of music sight reading for pianists is predictive of eye movement patterns.

The current project examined the effect of altering conventional features of score on the eye movement patterns of expert and non-expert music sight readers.

Participants sight read 10 specifically composed musical excerpts of 4 bars duration. These pieces were then played with the bar-lines removed, altered inter-note spacing and unpredictable beaming directions. Fixation characteristics were measured and compared.

Expert sight readers performed significantly faster than non-experts in both conditions: $\mathrm{p}<0.0001$. However, no significant difference in the number and duration of fixations was found between the normal and disrupted spacing conditions for either expert or nonexperts.

But, when fixation durations were subdivided into Long ( $>0.1$ seconds), Medium (0.1 $0.01)$ and Short ( $<0.01$ seconds) durations, eye movement differences between the levels of expertise were revealed. An overall expertise effect occurred for Long Duration Fixations: $\mathrm{p}<0.0001$ and the disrupted spacing caused the expert music sight readers to significantly reduce the number of Medium Duration Fixations: $p=0.0044$. The Short Duration Fixations did not significantly change.

These results showed that a more detailed analysis of the fixation data produced a better understanding of how eye movement patterns reveal processing differences between expert and non-expert music sight readers. 


\title{
Eye movement control during rapid reading: Word recognition and integration
}

\author{
Sarah J. White, Kayleigh L. Warrington, Marina Soltan, Victoria A. McGowan, Kevin B. \\ Paterson \\ University of Leicester, United Kingdom \\ s.j.white@le.ac.uk
}

Two experiments are presented that examined the nature of eye movement control during rapid reading compared to reading for comprehension. In Experiment 1 readers scanned sentences for a specific topic and in Experiment 2 readers skimmed sentences for general content. In Experiment 1 the frequency of a critical word was manipulated. There were additive effects of word frequency and task for first-pass reading times, such that there were immediate and localised effects of word frequency for both scanning and reading for comprehension. In contrast, for later measures there were larger effects of word frequency during reading for comprehension, indicating that integration processes may be limited during scanning. Experiment 2 examined the integration of sentential content with prior knowledge. Participants in Experiment 2 were medical students, and sentences were presented that were either accurate (consistent with their medical knowledge) or inaccurate. Importantly, re-reading measures showed larger effects of sentence accuracy during reading for comprehension than during skimming. Together these studies indicate that early word recognition mechanisms are similar during reading for comprehension and rapid reading, whereas integration processes are modulated by reading goals. The studies have important implications for the flexibility of mechanisms within models of eye movement control during reading. 
Talks

\title{
Eye movements: Social influences
}

\section{Room: HS 31}

\section{Attentional Effect of Social Cueing in Orientation Tasks}

\author{
Norah Neuhuber ${ }^{2}$, Lucas Paletta ${ }^{1}$, Michael Schwarz ${ }^{1}$ \\ 1 JOANNEUM RESEARCH Forschungsgesellschaft mbH, Austria \\ 2 University of Graz \\ norah_neuhuber@yahoo.de
}

Social cue based attention was initially attributed to observed gaze directions (Friesen \& Kingstone 1998). Other evidence for attentional effect is related to general evaluation considering combinations of gaze, head and body posture (Hietanen, 2002). When social cues and task-oriented stimuli are in competition, social cues are preferred (Birmingham, 2009). Attention from social cues increases with larger group size (Gallup et al., 2012).

This work investigated attentional influence by social cues, within the context of orientation tasks, which is relevant in the evacuation from buildings (Paletta, 2014). In the experimental study, images of an evacuation guidance system were displayed with appearance of guidance-related signs as well as with appearances of persons. To test the influence of group size, the number of depicted persons were varied (1 vs. 3). The scenes were observed under free-viewing conditions and in an orientation task.

The results indicate a differentiation between the two viewing conditions: Under freeviewing condition, participants preferably focus on social cues. In the orientation task, participants show a significant attention shift to guidance related signs. The attentional effect of social cues appears to be dependent on the instructed task and may not be generalized to everyday situations.

\section{Social salience-based prioritization does not transfer to oculomotor visual search.}

\author{
Alisha Siebold ${ }^{1}$, Matthew D. Weaver ${ }^{1}$, Mieke Donk ${ }^{2}$, Wieske van Zoest ${ }^{1}$ \\ 1 University of Trento, Italy \\ 2 Free University (VU) Amsterdam, Netherlands \\ alisha.siebold@unitn.it
}

Evidence suggests that socially relevant information (i.e., self-referential information) leads to attentional prioritization that is similar to prioritization based on stimulus-salience (e.g., Sui, Liu, Mevorach, \& Humphreys, 2013). However, these studies have used paradigms that are relatively insensitive to the characteristics of salience-based selection, including automatic and rapid attentional capture. The current study used an oculomotor visual search paradigm to investigate whether the time-course of prioritization based on selfrelevance resembles the time-course of physical salience. We report four experiments in which observers were asked to first perform a manual line-label matching task where associations were formed between orientation-lines (right- and left-tilted) and labels ("you" and "stranger"). Then participants made speeded saccades to one of the two lines without any task-instructions (Exp 1), to a dot probe located on one of the two lines (Exp 2), to the line that was validly cued by its associated label (Exp 3), or to the matching line-label pair (Exp 4). Prioritization of self-relevant information was only shown for the manual and visual matching-tasks, but not for visual search in Experiments 1-3. We argue that socialsalience has a qualitatively different effect on attentional processing from stimulus-salience, specifically acting upon higher-level perceptual decision making. 


\title{
The presence of others: Exploring the effects of a social context on eye movements
}

\author{
Manuel Oliva ${ }^{1}$, Diederick C. Niehorster ${ }^{1}$, Halszka Jarodzka ${ }^{2}$, Kenneth Holmqvist $^{1}$ \\ ${ }^{1}$ Lund University, Sweden \\ 2 Open University of the Netherlands \\ manuel.oliva@lucs.lu.se
}

Eyetracking research is normally conducted in individual settings, with participants performing alone. However, social psychology studies showed that the mere presence of others can have an influence on performance. Here we investigated the effect of a social context on eye movements and performance in two visual tasks. We used multiple eyetrackers and recorded participants in an individual condition or in groups of between 3 and 8 at the same time.

In the Antisaccade Task, we found that participants showed shorter reflexive prosaccade latencies when in groups, but longer antisaccade latencies compared to the individual condition. On the other hand, in a Visual Discrimination task where participants had to look at a peripheral target and report its orientation, there were no differences in saccade latencies between individual and group conditions. However, manual response times were slower in the group condition, although not significantly.

Our results suggest that the presence of others, even when not actively interacting with each other, can affect eye movement behavior. The effect on performance depends on whether the task involves reflexive or controlled behavior. We also discuss the implications of these results for educational research involving eye trackers.

\section{Eye Movements during Viewing of Simulated Crime Scenes Pictures by Crime Scene Investigators}

\author{
Murat Ozger, Timothy Hodgson, Jose Gonzalez-Rodriguez \\ University of Lincoln, United Kingdom \\ 12378768@students.lincoln.ac.uk
}

Perception and cognitive reasoning processes are an essential part of the crime scene investigation process, although they have rarely been addressed by empirical research. We used eye tracking to understand and evaluate these processes in crime scene investigators (CSIs). CSIs from a regional police force and undergraduate forensic science students and control subjects $(n=27)$ were asked to identify evidence and potential areas for investigation using mouse clicks in a series of static images of crime scenes presented on a computer monitor. Eye movements were tracked using an Eyelink 1000 system. Participants were also interviewed after completing the task about their perception and reasoning processes while viewing a playback of eye movements made whilst viewing each picture. CSIs were found to show significantly longer fixation durations overall (mean=334 ms(CSI), mean=297 ms (Student), mean=320 ms (Control)). Analysis of fixation characteristics on key interest areas indicated that differences between groups were only apparent in a subset of interest areas. These differences in eye movement strategies between skilled and unskilled viewers could aid in the design of educational or training programmes for CSIs in the future. 
Talks

\title{
Was it me? Recognition and Interpretation of Static and Dynamic Eye Movement Displays
}

\author{
Margot van Wermeskerken ${ }^{1,2}$, Damien Litchfield ${ }^{3}$, Tamara van $\operatorname{Gog}^{1,2}$ \\ ${ }^{1}$ Utrecht University, Netherlands \\ 2 Erasmus University Rotterdam, Netherlands \\ ${ }^{3}$ Edge Hill University, Lancashire, United Kingdom \\ vanwermeskerken@fsw.eur.nl
}

Replays of recorded eye movements are increasingly used within educational research to study or enhance learning processes. One of the underlying assumptions in such research is that observers can recognize displays of their own eye movements and meaningfully interpret eye movement displays of their own and someone else. The current study addressed this issue in two experiments. Participants' eye movements were recorded while they observed an image (Ilya Repin's "The unexpected visitor") under different instructions (cf. Yarbus, 1967). Subsequently, either static or dynamic displays of their own or someone else's eye movements were shown, overlaid on the stimulus image. Participants judged whether it were their own or someone else's eye movements and which instruction they reflected. Results indicated that participants performed slightly above chance in recognizing whether or not gaze displays were their own, but performed well above chance in interpreting the instructions they reflected, with the dynamic group outperforming the static. Being informed about the subsequent judgment tasks before their eye movements were recorded did not enhance participants' judgment performance. These findings are relevant for studies within the area of gaze (mis)understanding and for studies on learning and instruction that use eye movement replays to study or enhance learning. 


\title{
Microsaccades
}

\section{Room: HS 32}

\author{
Monocular microsaccades reflect eye dominance \\ Josselin Gautier ${ }^{1}$, Harold Bedell ${ }^{2}$ \\ ${ }^{1}$ Vision and Hearing Department, Anglia Ruskin University, United Kingdom \\ 2 College of Optometry, University of Houston, United States of America \\ josselin.gautier@anglia.ac.uk
}

We found recently that monocular microsaccades differ in frequency during fixation vs. a foveal discrimination task and, during discrimination, according to the visibility of the stimulus. These results lead to the hypothesis that monocular microsaccades might be triggered either to correct for small vergence errors or to recenter a target's image at the preferred retinal locus for fixation.

Using a stereoscope that allowed observers to align horizontal and vertical Nonius lines before each stimuli presentation, we recorded microsaccades during orientation discrimination of Landolt- $C$ targets while the fixation disparity was minimized. Landolt- $C$ targets were presented binocularly for $2 \mathrm{~s}$ at one of 5 levels of contrast. First, when fixation disparity is minimized, the occurrence of monocular microsaccades is reduced by a factor of 2-4 times, relative to a viewing condition without the prefixation of Nonius lines. Second, on successful trials of $\mathrm{C}$ discrimination, monocular microsaccades made by the motor dominant eye land significantly closer to the gap than binocular microsaccades or the monocular microsaccades made by the fellow eye.

The landing positions of the monocular microsaccades made by the two eyes during a visual task provide a representation of visual laterality or motor dominance and, potentially, of differential sensitivity between the two eyes.

\section{Visual categorization boosts microsaccade rate}

\author{
Yingdi Liu ${ }^{1}$, Junpeng Lao ${ }^{1}$, Nicolas Ruffieux ${ }^{1,2}$, Françoise Colombo ${ }^{2}$, Sébastien Miellet ${ }^{3}$, \\ Roberto Caldara ${ }^{1}$ \\ 1 University of Fribourg, Switzerland \\ 2 Fribourg State Hospital, Switzerland \\ ${ }^{3}$ Bournemouth University, United Kingdom \\ yingdi.irene@gmail.com
}

Our eyes are constantly moving to sample the visual world, even when maintaining steady fixation. Microsaccades are small involuntary eye movements produced during attempted fixation, preventing visual fading and sampling fine spatial information. Recently, it has been shown that low- and high-level visual information content directly modulates microsaccade rate. However, whether explicit visual categorization is critical to elicit microsaccades remains to be clarified.

To this aim, we measured the eye movements of a single case visual agnostic patient (BC), who suffers from a moderate global atrophy and a bilateral posterior white matter MRI hyperintensity in striate areas. BC is unable to explicitly or implicitly recognize shapes, colors, objects or scenes visually. As predicted, BC explored the visual inputs normally, while completely unable to recover the meaning of any of the images or single objects. In line with previous findings, BC's oculomotor behavior conformed to the low-level predictions made from visual saliency maps. However, although BC performed microsaccades in the same location than the controls, his rate was significant lower.

Our data show that object recognition enhances microsaccade rate. Microsaccades play thus a critical functional role in acquiring the fine-grained information necessary for the visual system to achieve effective categorization. 
Talks

\title{
The interdependence of microsaccades and its modulation by the visual context
}

\author{
Shlomit Yuval-Greenberg ${ }^{1,2}$, Roy Amit $^{2}$ \\ ${ }^{1}$ School of Psychological Sciences, Tel Aviv University, Israel \\ 2 Sagol School of Neuroscience, Tel Aviv University, Israel \\ shlomitgr@tau.ac.il
}

Research question: It was previously suggested that microsaccades are random events, and therefore the inter-microsaccadic intervals (IMSIs) between them follow an exponential distribution. In the current study we examined this hypothesis and tested whether the microsaccades are random or non-random events. We further hypothesis that the answer to this question varies according to the visual context.

Methods: In 3 experiments we recorded the gaze position of subjects while they viewed: A) Complete darkness, gray screen or natural scene (experiment 1); B) Checkerboard with low or high spatial frequency (experiment 2). C) Vertical or Horizontal lines with low or high spatial frequency (experiment 3).

Results: In all 3 experiments, the distribution of the IMSIs demonstrated a good fit to an exGaussian rather than an exponential distribution. Furthermore, the parameters of this exGaussian were greatly dependent on the visual background. With decreasing complexity of stimulation (darkness vs. complex scenes, or low vs. high spatial frequency) the distribution became more similar to an exponential distribution.

Interpretation: Microsaccades are interdependent and are not part of a random process as was previously thought. We hypothesis that that displacement of retinal image caused by microsaccades modulated the dynamic of consecutive microsaccades similarly to an external stimulation.

\section{Are microsaccade amplitudes overestimated in modern studies?}

\author{
Richard Andersson ${ }^{1}$, Dan Witzner Hansen ${ }^{1}$, Ignace T. C. Hooge ${ }^{3}$, Marcus Nyström ${ }^{2}$ \\ ${ }^{1}$ IT-University of Copenhagen, Denmark \\ 2 Lund University Humanities Lab, Sweden \\ ${ }^{3}$ Helmholtz Institute, Utrecht University, Netherlands \\ richard.andersson@humlab.lu.se
}

Microsaccade amplitudes in modern studies often exceed values from early microsaccade studies (1930s-70s) by a factor of five (up to $12 \mathrm{~min}$ arc vs up to 1 degree; as discussed by e.g. Collewijn \& Kowler, 2008). This increase coincides with a shift in tracking technology from contact lens methods (optical lever or coils) to non-invasive video-based tracking. Although video systems have higher noise levels, this can only partially explain the increase in amplitudes.

We use image processing algorithms to extract the features of the eye image corresponding to the tracked features of contact lens systems, video-based systems, and DPI systems, respectively. The position information from these eye structures differ in the overshoot component of a saccade. Whereas this overshoot is well-known for DPI data and handled accordingly, the overshoot of pupil-based systems (i.e., most video eye-trackers) appears not so explicitly treated. When examining the consequences of this overshoot for a popular microsaccade detection algorithm (Engbert \& Kliegl, 2003), we found that it often sets the offset of the microsaccade event near the peak of the overshoot rather than when the saccade has ended. This algorithm behavior overestimates the saccadic amplitude by a factor of two. 


\title{
Revising the link between microsaccade direction and the spatial cueing of attention
}

\author{
Susann Meyberg ${ }^{1,2}$, Petra Sinn ${ }^{3}$, Ralf Engbert ${ }^{3}$, Werner Sommer ${ }^{1,2}$ \\ ${ }^{1}$ Humboldt University at Berlin, Germany \\ 2 Berlin School of Mind and Brain, Germany \\ ${ }^{3}$ University of Potsdam, Germany \\ susann.meyberg@hu-berlin.de
}

Miniature saccades that occur during attempted fixation have been related to the orienting of attention: Covertly shifting attention away from central fixation results in a bias of microsaccade direction towards the peripheral target location. However, as a confound in most previous studies the target location was indicated by an asymmetrical cue (e.g. an arrow cue) with the task-relevant part of the cue (e.g. the tip of the arrow) being placed in the visual field congruent with the target location. Thus, here we tested the hypothesis that microsaccades align with the processing of the spatial cue instead of the attention shift. In a spatial cueing task with a symmetrical cue consisting of two arrowheads, we presented the task-relevant arrow either in the same visual field as the target location (congruent cue) or opposite to it (incongruent cue). With congruent cues that resemble previous cueing conditions, microsaccades showed the well-established bias towards the target location. With incongruent cues, however, microsaccades were clearly directed away from the target location, but towards the task-relevant cue. In conclusion, the effect of spatial cueing on microsaccade direction in large part seems to reflect a link between microsaccades and the selection and processing of the foveal cue. 


\section{Monday, August $17^{\text {th }}, 5.00 \mathrm{pm}-6.40 / 7.00 \mathrm{pm}$}

\section{Reading: Chinese}

\section{Room: HS 7}

\section{Modeling eye movement control during Chinese reading Xingshan Li \\ Institute of Psychology, Chinese Academy of Sciences, People's Republic of China lixs@psych.ac.cn}

Popular computational models of eye movement control during reading of alphabetic language experienced great challenges when modeling Chinese reading. In reading of alphabetic language, there are spaces between words to demarcate word boundaries. Thus, reader could perceive word boundaries with parafoveal vision and then move their eyes to the center of a word. Most models depend on this assumption when planning where to move their eyes. However, Chinese readers cannot do so because there are no spaces between words in Chinese text. To understand how Chinese readers control their eye movements, I will report the progress of building an eye movement model during Chinese reading. In the model, we assumed that Chinese readers plan their eye movements using a processing based strategy: they estimate the amount of information they can process at a given fixation, and then move their eyes to a location that carries novel information. To address the word segmentation problem, this model adopted some important assumptions of a model of word recognition and segmentation during Chinese reading ( $\mathrm{Li}$, Rayner \& Cave, 2009). The model successfully simulated many important findings during Chinese reading.

\section{Semantic information could mediate visual attention during spoken word recognition in Chinese}

Wei Shen, Qingqing Qu, Xingshan Li

Key Laboratory of Behavioral Science Institute of Psychology, Chinese Academy of Sciences, Beijing,

People‘s Republic of China

shenw@psych.ac.cn

Huettig and McQueen (JML, 2007) found that semantic information could not mediate the mapping between the speech signal and printed words upon hearing Dutch spoken target words. Given fundamental differences in linguistic properties across languages, we aimed to investigate whether semantically related words could be activated in spoken word recognition to affect visual attention shift in Chinese. In the printed word paradigm, participants heard a critical spoken word embedded in a neutral spoken sentence while looking at a visual display consisted of four printed words. The relationship between the critical spoken words and printed words was manipulated to be semantically related (a semantic competitor), phonologically related (a phonological competitor) or unrelated (two distractors). By calculating the fixation probability of each printed word, we found that the fixation probability of semantic competitors was significantly higher than distractors, which was inconsistent with the finding in Dutch. In addition, a significant phonological competitor effect was also observed. This study not only revealed that semantically related words of a spoken word could be activated to affect visual attention, but also made a contribution to the methodology of investigating the semantic processing of spoken words in spoken word recognition using the printed word paradigm. 


\title{
Effects of Aging and the Visual Quality of Text on Eye Movements While Reading Chinese
}

\author{
Kevin B. Paterson ${ }^{1}$, Lin $\mathrm{Li}^{2}$, Sha $\mathrm{Li}^{2}$, Simon P. Liversedge ${ }^{3}$, Jingxin Wang ${ }^{2}$ \\ ${ }^{1}$ College of Medicine, Biological Sciences and Psychology, University of Leicester, United Kingdom \\ ${ }_{2}$ Academy of Psychology and Behavior, Tianjin Normal University, People's Republic of China \\ ${ }^{3}$ School of Psychology, University of Southampton, United Kingdom \\ kbp3@le.ac.uk
}

Age-related changes in eye movement behavior are well-established for alphabetic languages. Moreover, older readers appear to compensate for increased reading difficulty by employing a risky reading strategy in which they more often infer the identities of upcoming words based on only partial information and so skip words more frequently. Effects of aging on reading for visually-complex logographic languages like Chinese are largely unknown. Therefore, the present research examined the eye movements of young and older adult readers of Chinese. These experiments also investigated the resilience of readers to changes in visual quality by presenting a specific target word (Experiment 1), or all characters in a sentence (Experiment 2), normally or faint (with reduced visual contrast). Broadly typical patterns of age-related reading difficulty were observed. But, rather than engaging in risky reading, the older adults read more cautiously than the younger adults and skipped words less frequently. They also had more difficulty when text was faint, indicating their reading abilities are less resilient to variation in visual quality. The findings suggest adult age differences in reading behavior are language-specific and that older Chinese readers adapt to the specific challenges of their writing system by reading more carefully.

\section{The use of probabilistic lexicality cues for word segmentation in Chinese reading

\author{
Chuanli Zang ${ }^{1}$, Yongsheng Wang ${ }^{1}$, Xuejun Bai ${ }^{1}$, Guoli Yan ${ }^{1}$, Denis Drieghe ${ }^{2}$, Simon P. \\ Liversedge ${ }^{2}$ \\ ${ }^{1}$ Tianjin Normal University, People's Republic of China \\ 2 University of Southampton, United Kingdom \\ dedipsy@yahoo.com
}

Even though Chinese is an unspaced, character based language with no clear demarcation of word boundaries, effects of word based processing have been observed, raising questions on how Chinese readers segment character strings into words as they read. In an eye tracking experiment we examined whether Chinese readers were sensitive to how often a Chinese character appears as a single character word versus the first character in a two character word, and whether readers use this information to segment words and adjust the amount of parafoveal processing of subsequent characters. Participants read sentences containing a two-character target word with its first character more or less likely to be a single character word. The boundary paradigm was used. The boundary appeared between the first and second character of the target and we manipulated whether readers saw an identity or a pseudocharacter preview of the second character of the target. Results showed reduced preview benefit from the second character when the first character was more likely to be a single character word suggesting that Chinese readers use probabilistic combinatorial information about the likelihood of a Chinese character being single-character word or a two-character word online to modulate the extent of parafoveal processing. 


\title{
The upper halves of Chinese characters are more advantageous than the lower halves for word identification during reading
}

\author{
Hui Chi ${ }^{1,2}$, Guoli Yan ${ }^{1}$, Zhu Meng ${ }^{1}$, Chuanli Zang ${ }^{1}$, Simon P. Liversedge ${ }^{2}$ \\ 1 Tianjin Normal University, People's Republic of China \\ 2 The University of Southampton, United Kingdom \\ hc7g14@soton.ac.uk
}

Previous studies have argued that upper halves of Chinese characters facilitate character and word recognition more than lower halves (Chou, 1930; Tsao \& Wang, 1983). However, these studies did not control many factors known to affect reading times (e.g., number of strokes, character structure, etc.), nor did they employ eye movement methodology to measure the time course of effects. In our experiment, participants' eye movements were recorded when they read single sentences with only the upper halves of characters presented, only the lower halves of characters presented, or with the entire sentence presented. We also included a one-character target word manipulated for the orthographic ambiguity of its upper or lower half. Targets were either balanced (so that the same number of characters could be formed from the upper and lower half), or were unbalanced (so that more characters could be formed from the upper half than the lower half, or vice-versa). Global reading times showed an advantage for whole, over upper, over lower half presentations. A similar pattern occurred for the target word, suggesting that the upper half advantage was not modulated by orthographic ambiguity. A follow-up experiment will rule out the possibility that contextual spillover caused target word effects.

\section{Binocularity and the reading of Chinese characters, words and text Yi-ting Hsiao, Richard Shillcock \\ The University of Edinburgh, United Kingdom \\ N.Y.Hsiao@ed.ac.uk}

How does binocularity mediate the reading of Chinese characters, words, and text? We report (a) the analysis of movements within fixations in a very large eye-movement database of the binocular reading of multiline Chinese text; (b) an experiment in which fixations were haploscopically-controlled to present separate parts of Chinese characters and words to particular hemifields in each eye. For the eye-tracking study, we report the overall direction and extent of movement between the beginning and end of the fixation in each eye for several hundred thousand binocular fixations. The data show significant qualitative and quantitative differences between Chinese and the other four languages, including semitic (right-to-left) in our corpus. We interpret these differences in terms of different roles for the right and left eye with respect to reading direction, and in terms of the greater visual complexity of Chinese orthography. The results for the haploscopic experiment show differential reliance on the processing of the contralateral and ipsilateral hemifields, in the two eyes, for both single "phonetic complex" (two-part) characters (with distinct left and right parts) and for two-character words. In both, there is greater reliance on the processing in the contralateral hemifield. 


\title{
Visual search
}

\author{
Room: HS 30
}

\section{Manipulating lag-2 revisits during visual search reveals evidence for mixed influences for fixation termination during visual search.}

\author{
Tamaryn Menneer, Hayward J. Godwin, Erik D. Reichle \\ University of Southampton, United Kingdom \\ t.menneer@soton.ac.uk
}

During visual search, observers often fixate a target stimulus, move the eyes to a different stimulus, and then return to the target on the next fixation (e.g., McCarley et al., 2003; Peterson et al., 2007; Wienrich et al., 2009). This pattern has been referred to as a lag-2 revisit (Peterson et al., 2001), and occurs fairly frequently in visual search ( $20 \%$ of trials). Our aim was to better understand why lag- 2 revisits occur by determining some of the influences behind them. We found that the probability of lag-2 revisits occurring increased as the number of target-similar stimuli increased in search displays, and that these revisits were preceded by a brief fixation on the intervening distractor stimulus. We developed a computational model, called the Efficient Visual Sampling (EVS) model, to simulate our findings in terms of fixation durations and fixation locations, and to identify the parameters that are necessary to give rise to lag-2 revisits during visual search. Our findings provide evidence for both global- and local-level influences on eye-movement behavior.

\section{Visual Search for Transparent and Opaque Overlapping Objects in Depth}

\author{
Hayward J. Godwin ${ }^{1}$, Tamaryn Menneer ${ }^{1}$, Simon P. Liversedge ${ }^{1}$, Kyle R. Cave ${ }^{2}$, Nick S. \\ Holliman $^{3}$, Nick Donnelly ${ }^{1}$ \\ 1 University of Southampton, United Kingdom \\ 2 University of Massachusetts Amherst, United States of America \\ 3 University of York, United Kingdom \\ hg102@soton.ac.uk
}

Standard visual search tasks present participants with isolated images that do not overlap with one another on a single depth plane. Here, we had two goals: first, to examine search performance and eye-movement behaviour for overlapping images; second, to determine whether performance is improved when depth information is available. Participants searched displays containing coloured polygons with varying levels of overlap. The polygons were opaque (Experiment 1) or transparent (Experiment 2). For half of the participants, the objects were presented upon different depth planes to one another, and for the other half, the objects were presented on a single depth plane. Increasing overlap impaired performance (increased RTs, decreased accuracy), and increased the number and duration of fixations. Both accuracy and RTs were higher for the transparent displays versus the opaque displays. Depth did not influence performance in transparent displays, but in the opaque displays depth did reduce RTs and the number of fixations made for the most difficult overlap conditions. Overall, depth appears to have limited benefits to performance, while overlap impairs performance mostly when the objects are opaque. When the objects are transparent, object identity information is available, but more time is needed to determine each object's identity. 
Talks

\title{
Stuck on semantics: Automatic processing of object-scene incongruities modulates ongoing gaze behavior.
}

\author{
Tim H. W. Cornelissen, Melissa Le-Hoa Võ \\ Goethe University Frankfurt, Germany \\ cornelissen@psych.uni-frankfurt.de
}

People have an amazing ability to identify objects and scenes within only a glimpse of an eye. How automatic is this scene and object identification? Are scene and object semantics -let alone their semantic congruity- processed to a degree that modulates ongoing gaze behavior even if they are irrelevant to the task at hand?

Objects that do not fit the semantics of the scene (e.g. a toothbrush in an office) are typically fixated longer and more often than objects that are congruent with the scene context. In the current study, we overlaid a letter $\mathrm{T}$ on photographs of indoor scenes and instructed fourteen participants to search for it. Some of these background images contained scene incongruent objects.

Despite their lack of relevance to the search, we found that subjects made longer individual fixations on, and spent more time in total looking at incongruent compared to congruent objects in the same position of the scene.

Individual fixation durations are quite insensitive to strategic influence. We argue that when we view natural scenes, scene and object identities are processed automatically. Moreover, even when irrelevant to our current search, a semantic mismatch between scene and object identity can modulate ongoing eye movement behavior.

\section{Alasdair D. F. Clarke, Amelia R. Hunt \\ University of Aberdeen, United Kingdom a.clarke@abdn.ac.uk}

A strategic failure in eye movements that extends to other modalities

Morvan and Maloney (2012) provide striking evidence that human observers do not reliably fixate locations that maximise their probability of detecting a target. In their study, observers failed to adjust their choice of where to fixate when the possible target locations were far apart versus close together. We were interested in whether this failure to take task difficulty and probable success into account when making a decision could be considered specific to the context of eye movements and detection of targets, or a larger problem pervading human decisions in general. Saccadic eye movements are rapid, energy-efficient, and frequently not under voluntary control, so it is reasonable to suspect that the decisional processes involved would not generalize to other modalities. We first replicate their results, and then demonstrate very similar failures in decision making about a) where to stand to throw a beanbag to a target, and $b$ ) whether to try and memorize one or two numbers for later report, suggesting this failure in optimal behaviour may pervade human decisions in general. This failure can be characterized as an inability to allocate resources efficiently when faced with multiple competing goals. 


\title{
Mirror blindness in visual search for unfamiliar shapes and faces.
}

\author{
Stefanie I. Becker ${ }^{1}$, Dustin Venini ${ }^{1}$, James D. Retell ${ }^{1}$, Intan Wardhani ${ }^{1}$, Jeremy M. Wolfe ${ }^{2}$ \\ 1 The University of Queensland, Australia \\ 2 Brigham and Women's Hospital, Harvard Medical School, Boston, United States of America \\ s.becker@psy.uq.edu.au
}

In previous studies, we showed that search for a mirror-reversed target takes longer because observers fail to recognise the target and continue searching even when they have already looked at the target. This failure to recognize the target item as the search target was specific to mirror-reversed targets and did not occur for differently-oriented (e.g., vertically flipped, or 90 degrees rotated) targets. In the present study we tested observers' knowledge about the target position by masking all search items after observers had fixated on the target, and asking them to report the target location. In addition to the unfamiliar simple shapes of our prior experiments, we also tested differently oriented faces. Observers had no reliable knowledge about the target position, even though they had fixated on the target before it was masked. Among the factors modulating awareness were (1) whether observers had fixated on the target more than once during a trial, and (2) whether observers had been fixating on the target when the masks appeared. In conclusion, the results show that mirror blindness is a robust phenomenon, and that we can indeed genuinely fail to recognise a mirror-reversed target even when we are directly looking at it.

\section{Effectiveness and use of strategy in visual search increase during adolescence}

\author{
Rudolf Burggraaf ${ }^{1,2}$, Jos N. van der Geest ${ }^{1}$, Maarten A. Frens ${ }^{1,3}$, Ignace T. C. Hooge ${ }^{2}$ \\ ${ }^{1}$ Erasmus MC, The Department of Neuroscience, Rotterdam, Netherlands \\ 2 Helmholtz Institute and Department of Experimental Psychology, Neuroscience \& Cognition, Utrecht \\ University, Netherlands \\ ${ }^{3}$ Erasmus University College, Rotterdam, Netherlands \\ r.burggraaf@erasmusmc.nl
}

A child's brain is changing tremendously during adolescence, and planning and strategy are among the importantly developing abilities. We hypothesized that visual search will also change during this period of life. In particular, we are interested in effectiveness (i.e. response time and accuracy) and the efficient use of selection and strategy to minimize dwell time on obvious non-targets and to prevent skipping possible targets or revisiting elements. A large group of adolescents ( $\mathrm{N}=128$; age: $12-19$ years; $54 \%$ male) participated in a visual search experiment. The experiment was composed of 144 target present or absent trials (binary choice; $50 \%$ prevalence). Each trial showed a stimulus display with 36 Gabor patches placed on a hexagonal grid. The target consisted of a vertical oriented element with a high spatial frequency. Non-targets differed from the target in spatial frequency and/or orientation.

Accuracy and number of dwells per display did not change with age, but dwell times and the proportion of revisited elements decreased significantly. Older children showed more regular scan paths, making more 'reading' and 'pendulum' scans over the search displays. We conclude that during adolescence children become more effective searchers and increasingly use efficient search strategies. 
Talks

\title{
Eye movements and reading: Clinical
}

Room: HS 31

\section{Phonological Processing and Oculomotor Control Differentially Modulate Reading Comprehension Deficits in Schizophrenia and Developmental Dyslexia}

\author{
Veronica M. Whitford ${ }^{1}$, Nicholas Ostapchuk ${ }^{2}$, Debra A. Titone ${ }^{2}$, Gillian A. O'Driscoll ${ }^{2}$ \\ 1 The University of Western Ontario, Canada \\ ${ }^{2}$ McGill University, Canada \\ vwhitfor@uwo.ca
}

\begin{abstract}
Although clinically distinct disorders, schizophrenia and developmental dyslexia both involve disrupted functions that contribute to reading, including language processing, visual perception, and oculomotor control. This suggests that reading may be similarly affected in both disorders. Recently, we demonstrated that impaired reading comprehension in schizophrenia was associated with deficits in higher-order oculomotor control (antisaccade performance), but not with deficits in phonological processing (Whitford et al., 2013). Here, we examined whether a similar association between higher-order oculomotor control and reading comprehension would be found in dyslexia. Readers with dyslexia $(n=19)$ and demographically-matched controls $(n=17)$ were administered the antisaccade test to measure higher-order oculomotor control along with the Comprehensive Test of Phonological Processing (CTOPP) to measure phonological processing and the Nelson-Denny Reading Test (NDRT) to assess reading comprehension. Dyslexia participants had impaired antisaccade performance as well as deficits in phonological processing (CTOPP) and reading comprehension (NDRT). However, in dyslexia antisaccade performance was not associated with reading comprehension while measures of phonological processing (i.e., rapid letter naming) were. Thus, our results suggest that higher-order oculomotor control and phonological processing are similarly impaired in schizophrenia and dyslexia, but these factors contribute differently to reading comprehension deficits in the two disorders.
\end{abstract}




\title{
Eye Movements and Sentence Processing in Adults with Autism Spectrum Disorder
}

\author{
Philippa Lucy Howard, Valerie Benson, Simon P. Liversedge \\ University of Southampton, United Kingdom \\ PLH1G11@soton.ac.uk
}

Text comprehension is often poor in readers with autism spectrum disorder (ASD). However, little is known about underlying linguistic processing differences that underpin such poor comprehension. In two experiments, the eye-movements of adults with and without ASD were monitored as they read sentences. In Experiment 1, lexical identification was examined by manipulating word frequency. In Experiment 2, parsing preferences and the speed with which world knowledge was used during reading, were examined through the use of sentences containing ambiguous prepositional phrases. Both the control and ASD group showed normal word frequency effects that were evident during the first fixation on the target word (Experiment 1). Disruption to reading was first evident for both groups in single fixation durations on the target word that disambiguated the prepositional phrase attachment ambiguity (Experiment 2). The magnitude and immediacy of this disruption did not differ between groups. ASD participants however, engaged in more second pass reading, in both experiments. This may indicate that ASD readers took longer to make a judgement that their interpretation of the sentence was reasonable. Overall, the results indicate that TD and ASD groups engage in comparable processes to identify words, hold similar syntactic preferences and use world knowledge online.

\section{Mild Alzheimer's disease: evidences from the analysis of eye movement during reading

\author{
Gerardo Fernández ${ }^{1}$, David Orozco ${ }^{2}$, Liliana Raquel Castro ${ }^{3}$, Marcela Schumacher $^{3}$, \\ Osvaldo Enrique Agamennoni ${ }^{3,4}$ \\ 1 CONICET, Buenos Aires, Argentina \\ 2 Clinica Privada Bahiense, Buenos Aires, Argentina \\ 3 Universidad Nacional del Sur, Buenos Aires, Argentina \\ 4 Comisión de Investigaciones Científicas, Buenos Aires, Argentina \\ gerardofernandez480@gmail.com
}

We analyzed the eye movement behavior of thirty five healthy readers (Controls) and thirty five patients with probable Alzheimer disease (AD) during reading Spanish regular and highly-predictable sentences. We used lineal mixed models to analyze eye movement information. The cloze predictability of words $\mathrm{N}-2, \mathrm{~N}-1$, and $\mathrm{N}+1$ exerted an influence on reader's gaze duration. The predictabilities of preceding words in highly-predictable sentences served as task-appropriate cues that were used by Controls readers. In contrast, these effects were not present in AD patients. For analyzing retrieval memory we determined, for each sentence, a word at which predictability of words grows significantly. In Controls, changes in predictability significantly affected fixation duration along the sentence; noteworthy, these changes did not affect fixation durations in AD patients. Our results suggest that Controls used stored information of familiar texts for enhancing their reading performance and imply that contextual-word predictability, whose processing require memory retrieval, only affected reading behavior in healthy subjects. In AD, this loss might reveal impairments in brain areas such as those corresponding to working memory and memory retrieval. These findings might be relevant for expanding the options for the early detection and monitoring in the early stages of AD. 
Talks

\section{Is "Pervasive ocular tremor of Parkinson's" actually neither pervasive, nor ocular, nor uniquely parkinsonian?}

Michael R. MacAskill ${ }^{1,2}$, Daniel J. Myall ${ }^{1}$, Toni L. Pitcher ${ }^{1,2}$, Masayuki Watanabe ${ }^{1}$, Tim J. Anderson $1,2,3$

${ }^{1}$ New Zealand Brain Research Institute, Christchurch, New Zealand

2 University of Otago, Christchurch, New Zealand

${ }^{3}$ Department of Neurology, Christchurch Hospital, New Zealand michael.macaskill@nzbri.org

A characteristic tremulous oscillation of the eyes in Parkinson's disease was recently described, which could provide the long-sought objective, sensitive and specific diagnostic sign for the disorder. Vigorous debate has ensued as to whether this "ocular tremor" is a real phenomenon or simply an artefact secondary to body tremor. A large, definitive study able to either confirm or reject the original finding was required.

We examined 681 video-oculography recordings from a longitudinal study of 188 Parkinson's patients and 66 controls. Fourier analysis was used to detect oscillations not only in raw pupil image position data but also in corneal reflection signals, which can correct for head motion.

We detected oscillations in raw position signals which were similar to those in the original report. They were strongly related to clinical somatomotor tremor severity. They were not, however, present universally in patients and the frequency (if not power) distribution was similar in controls. Crucially, these oscillations were abolished when subtracting the corneal reflection signal from the pupil signal in order to correct for head motion.

We found strong evidence that the postulated purely ocular tremor of Parkinson's does not exist, other than as an artefact arising from vestibulo-ocular compensation for somatic tremor. 


\title{
Eye movements: Conflict and inhibition
}

\author{
Room: HS 32
}

\section{Oculomotor dominance in multitasking: Conflict resolution in cross-modal action}

\author{
Aleksandra Pieczykolan, Lynn Huestegge \\ University of Wuerzburg, Germany \\ aleksandra.pieczykolan@uni-wuerzburg.de
}

In daily life, eye movement control usually occurs in the context of concurrent action demands in other effector domains. However, little research has focused on understanding how such cross-modal action demands are coordinated, especially when conflicting information needs to be processed conjunctly in different action modalities. In two experiments, we address this issue by studying vocal responses in the context of spatially conflicting eye movements (Experiment 1 ) and in the context of spatially conflicting manual actions (Experiment 2, under controlled eye fixation conditions). Crucially, a comparison across experiments allows us to assess resource scheduling priorities among the three effector systems by comparing the same (vocal) response demands in the context of eye movements in contrast to manual responses. The results indicate that in situations involving response conflict, eye movements are prioritized over concurrent action demands in another effector system. This oculomotor dominance effect corroborates previous observations in the context of multiple action demands without spatial response conflict. Furthermore, and in line with recent theoretical accounts of parallel multiple action control, resource scheduling patterns appear to be flexibly adjustable based on the temporal proximity of the two actions that need to be performed.

\section{Talking While Looking: On the Modularity of the Oculomotor Control System}

\author{
Lynn Huestegge ${ }^{1}$, Aleksandra Pieczykolan ${ }^{1}$, Iring Koch ${ }^{2}$ \\ 1 Würzburg University, Germany \\ 2 RWTH Aachen University, Germany \\ Lynn.Huestegge@psych.rwth-aachen.de
}

The idea that the human mind can be divided into distinct (but interacting) functional modules is an important presupposition in many theories of cognition. While previous research on modularity predominantly studied input domains (e.g., vision), the present study examined output domains, with a focus on the oculomotor system. We asked to what extent oculomotor control is encapsulated (i.e., immune to influence from other domains) by studying determinants of interference between simultaneous action demands that were previously considered relatively distinct (oculomotor and vocal responses). To examine the degree of encapsulation, we compared single- vs. dual-response performance triggered by single stimuli. Experiment 1 addressed the role of stimulus modality under dimensionally overlapping response requirements (stimuli and responses were spatial and compatible throughout). In Experiment 2, we manipulated the presence of dimensional overlap across responses. Substantial performance costs associated with dual-response (vs. single-response) demands were observed across response modalities, conditions, and experiments. Dimensional overlap combined with shared spatial codes across responses enabled response-code priming (i.e., beneficial crosstalk between vocal and oculomotor action domains). Overall, the results are at odds with the idea of strong encapsulation of the oculomotor control system and show how processing content determines the extent of interdependency between output domains. 
Talks

\title{
Audiovisual intgration of an irrelevant distractor
}

Jessica Heeman ${ }^{1}$, Tanja C.W. Nijboer ${ }^{1,2,3}$, Nathan Van der Stoep ${ }^{1}$, Stefan Van der Stigchel ${ }^{1}$

${ }^{1}$ Department of Experimental Psychology, Helmholtz Institute, Utrecht University, Netherlands

2 University Medical Center Utrecht, Department of Neurology, Utrecht, Netherlands

${ }^{3}$ Rudolf Magnus Institute of Neuroscience and Center of Excellence for Rehabilitation Medicine,

University Medical Center Utrecht and Rehabilitation Center De Hoogstraat, Utrecht, Netherlands j.heeman@uu.nl

When executing eye-movements to a target, the presence of an irrelevant distracting stimulus influences the saccade endpoint. The present study investigated the influence of auditory, visual and audiovisual distractors close or remote from a target on the endpoint error of eye-movements. Participants made eye-movements to a visual target in the presence of one uni- or bimodal distractor. There were two main findings. First, interference evoked by an audiovisual distractor was stronger, beyond statistical facilitation, compared to a unimodal distractor, but only for close distractors. This indicates that integration of auditory and visual distractor information depended on the distance between target and distractor. This might be explained by the fact that close distractors fell within the spotlight of attention induced by preparation of eye-movements to the target. Previous studies have indeed shown that endogenous attention facilitates multisensory integration. Our second finding is that unimodal auditory distractors did not influence saccade endpoints, regardless of location. We hypothesize this is due to spatial ventriloquism or high target-predictability in this experiment. Our study therefore indicates that bimodal distractors do not necessarily evoke stronger competition in the oculomotor system, but that the strength of the competition depends on the spatial layout of a visual scene.

\section{Event structure influences on object accessibility during competitive retrieval}

\author{
Glenn Patrick Williams ${ }^{1}$, Anuenue Kukona ${ }^{2}$, Yuki Kamide ${ }^{1}$ \\ 1 University of Dundee, United Kingdom \\ 2 De Montfort University, United Kingdom \\ glenn88.williams@gmail.com
}

When objects are located in two distinct locations (e.g. rooms) we segment the ongoing stream of events into separate event models (Radvansky, 2012). In two eye-tracking experiments, we established the role of event segmentation on object accessibility during discourse processing. In Experiment 1, participants heard narratives describing movement of a target to a goal while viewing two rooms separated by a doorway that could be located between (two-event condition) the target and goal, or not (one-event condition). On re-mention of the target, we found fewer looks to the goal in the two-event condition, suggesting event segmentation can decrease accessibility for a target. In Experiment 2, participants viewed a quadrant containing a target, a semantically-related competitor, and two unrelated distractors (see Yee \& Sedivy, 2006). Participants then heard narratives describing two of the objects located in one location, and the other two in another. On re-mention of the target object, we found more looks to the target and fewer looks to the competitor if they were in separate locations (vs. the same), suggesting event segmentation can increase accessibility for a target. Together, our results suggest a differential role for event segmentation on accessibility for same/different object representations during competitive retrieval. 


\title{
The role of imagined perspective in route description encoding and map representations: evidence from eye tracking.
}

Fabio Parente, Alastair D. Smith, Ruth Filik

School of Psychology, The University of Nottingham, United Kingdom Ipxfp1@nottingham.ac.uk

Eye movements offer a window on the way we allocate attention to different features of linguistic or visual representations of environments, and on the creation of mental representations. In our experiments we manipulated the type of relational terms used in route descriptions (egocentric "left/right" vs allocentric cardinal terms), and the instructions provided ("Imagine a first-person [egocentric] vs. bird's-eye [allocentric] view of the environment described."), observing their effect on reading behaviour during description encoding and on recall of environmental features (i.e. landmarks located at different locations). While navigationally salient landmark words were fixated significantly longer than non-salient ones during encoding of egocentric descriptions, the use of cardinal terms produced no such difference. Additionally, encoding a cardinal description while maintaining an egocentric imagined perspective generally decreased map drawing performance while increasing selfreported task difficulty.In an ongoing follow-up study we have replaced the map drawing task with a map recognition task, allowing us to also acquire ocular measures during the recall phase. Our goal is to better understand how bottom-up and top-down factors (linguistic manipulations vs imagined spatial perspectives) influence the way we acquire spatial information from descriptions and maps, and how we use the resulting mental representations to navigate our surroundings.

\section{Multiple diffusion models to compare saccadic and manual responses for Inhibition of return}

\author{
W. Joseph Maclnnes
}

Higher School of Economics, Russian Federation jmacinnes@hse.ru

Cuing a location in space produces a short lived advantage in reaction time to targets at that location. This early advantage, however, switches to a reaction time cost and has been termed Inhibition of return (IOR). IOR behaves differently for different response modalities suggesting that it may not be a unified effect. Multiple diffusion models are presented of saccadic and manual reaction time for cuing experiments with random CTOA and probe locations. Diffusion models can generate accurate distributions of reaction time data by modelling a response as a build-up of evidence towards a response threshold. We test a number of IOR theories by adapting this model to allow for changes in signal strength over time. We suggest that if saccadic and attentional IOR are based on similar processes, then differences in distribution will be best explained by adjusting parameters values such as signal and noise within the same model structure. Alternately distinct causes of IOR would be explained best by different model structure such as the number of parameters or number of competing signals. Best fit models from experimental data are shown to best fit as different model structures suggesting separate underlying causes for saccadic and manual IOR. 
Tuesday, August $18^{\text {th }}, 8.50 / 9.10$ am -10.30 am

What the eyes tell us about bilingual language processing

Room: HS 7

How second language experience impacts first and second language reading: Evidence from several eye movement tasks in younger and older adults

Debra A. Titone ${ }^{1}$, Veronica M. Whitford ${ }^{2}$, Irina Pivneva ${ }^{1}$

${ }^{1}$ Psychology, Centre for Research on Brain, Language \& Music, McGill University, Canada

2 Psychology, Brain \& Mind Institute, Western University, Canada debra.titone@mcgill.ca

Eye movement studies have informed how bilinguals, as a group, process language. Less clear is how differences among bilinguals in second language experience affect first and second language (L1, L2) reading. We addressed this across several studies of younger adults (ages: 18 - 35) focussing on: L1 and L2 word frequency and contextual predictability effects during paragraph reading; cross-language competition during sentence reading; and global measures of sentence reading and perceptual span using a gaze-contingent moving window task.

The data across studies suggest that greater L2 experience facilitates L2 reading but impedes L1 reading (reflected by gaze durations on language-unique words, the size of frequency effects), and similarly affects global measures of sentence reading (reflected by words read per minute, perceptual span). However, greater L2 experience in younger adults does not as reliably predict cross-language effects arising from orthographic density and interlingual homograph status.

Finally, new work with older adults (ages: 60 - 87) using the same tasks, suggests a blunted effect of L2 experience, presumably because older readers have greater overall life-long experience with language, particularly the L1. Taken together, the findings suggest that L2 experience systematically modulates L1 and L2 reading, but may have a reduced impact with increased age. 


\author{
Cognate effects in L1 and L2 natural reading \\ Eva Van Assche ${ }^{1}$, Uschi Cop ${ }^{1}$, Denis Drieghe ${ }^{2}$, Wouter Duyck ${ }^{1}$, Robert Hartsuiker ${ }^{1}$ \\ ${ }^{1}$ Ghent University, Belgium \\ 2 University of Southamption, United Kingdom \\ eva.vanassche@ugent.be
}

An important issue in bilingualism research concerns the question of whether reading a word activates lexical representations in both languages, or in only the contextually relevant target language. Many studies on words out-of-context have shown that representations from both languages activated in parallel. More recent studies presenting words in sentence context have shown that the language of the preceding words is insufficient to restrict lexical access to words of the target language, even when reading in the native language.

We studied the effects of identical cognate status (e.g., Dutch-English identical cognate ringring) and cross-lingual orthographic overlap on natural reading of nouns. Dutch-English bilinguals read an entire novel in L1 and L2 while their eye movements were monitored. Early reading time measures such as first fixations showed effects of orthographic overlap in L1 and L2 indicating that words with more cross-lingual overlap were read faster. Later reading time measures showed effects of identical cognate status in L2 meaning that identical cognates were read faster than other words. These results show that the linguistic context provided by a novel is insufficient to constrain cross-language activation.

\title{
Bilingual listeners use phonetic cues to anticipate codeswitches
}

\author{
Melinda Fricke, Judith F. Kroll, Paola E. Dussias \\ Pennsylvania State University, United States of America \\ mdf18@psu.edu
}

Research on bilingual auditory word recognition has demonstrated robust competition from the non-target language: when a target picture (e.g., a pot) is embedded in a display containing an interlingual distractor (a duck; pato, in Spanish), bilinguals' eye movements reveal an inability to ignore the interlingual distractor. However, ongoing corpus work in our lab indicates that during spontaneous codeswitching, speakers produce anticipatory phonetic cues that could allow listeners to predict when a codeswitch is about to happen (Fricke, Kroll, \& Dussias, in prep). In the present study, we presented target words in codeswitched sentences either with or without subtle phonetic modulations modeled on the earlier corpus work: listeners were instructed to "click on the picture of the [Spanish target]", and in the "cued" condition, the voice onset time in "click" and in "picture", and the speech rate of the carrier phrase were all decreased slightly. Preliminary results indicate faster activation of the target word and decreased interference from the interlingual distractor on trials in which naturalistic anticipatory phonetic cues signaling the codeswitch are present. The results demonstrate that even though activation of the non-target language appears inevitable, experienced codeswitchers can exploit subtle phonetic information to optimize their regulation of that activation. 
Talks

\section{Found in translation: what eye movement data can tell us about the development of formulaic language in bilingual speakers

\author{
Kathy Conklin, Gareth T. Carrol
} \\ University of Nottingham, United Kingdom \\ kathy.conklin@nottingham.ac.uk}

Highly familiar "formulaic" sequences (e.g. idioms) are recognised quickly and understood effortlessly by native speakers but remain problematic for non-native speakers even at high proficiency levels. We explore how well-known idioms are processed in the L2 by advanced learners (L1 Swedish). We compare English only idioms with translations of Swedish idioms (which do not make sense in English) to see how L1 and L2 knowledge interact, in terms of form (recognition/activation of individual words) and understanding of phrase level meaning.

With regards to form recognition, native speakers showed the expected "idiom advantage" for known English idioms (higher likelihood of skipping, shorter early duration measures). Non-native speakers showed this effect for translated L1 idioms but not L2 ones.

Late measures (total reading time, regression duration) show that advanced non-natives had no difficulty understanding figurative meanings of translated L1 idioms or L2 idioms. English native speakers showed considerable disruption for the translated Swedish idioms. This pattern indicates that bilinguals use existing L1 knowledge and learned L2 knowledge to access phrase-level meaning.

Eye-tracking reveals how L1 knowledge can facilitate both form and meaning activation in the L2, but that development of "nativelike" L2 processing can be difficult for bilinguals.

\section{Cross-language parafoveal semantic preview benefit for Korean-Chinese bilingual readers

\author{
Ming Yan ${ }^{1}$, Aiping Wang ${ }^{2}$ \\ 1 University of Potsdam, Germany
} \\ 2 Beijing Normal University, People's Republic of China mingyan@uni-potsdam.de}

Parafoveal processing has been well documented for orthographic and phonological information in the past decades whereas evidence for semantic preprocessing in alphabetic scripts has been traditionally elusive. In the present study, we aimed at testing crosslanguage semantic preview effects using the gaze-contingent boundary paradigm. Eye movements of 47 native Korean readers who learned Chinese as a second language were recorded by an EyeLink 2000 eye-tracker during the reading of Chinese sentences. Each Chinese target word was paired with three Korean preview words: a cognate translation of the target, a semantically related non-cognate word and an unrelated word. Together with a highly significant cognate preview effect, more importantly, we observed reliable facilitation in processing of the target word from semantically related parafoveal previews in early and late processing measures. We argue that a relatively transparent orthography-to-phonology mapping in Korean leads to faster phonological decoding which in turn facilitates access to semantics during the short fixation periods. Results from the present study provide evidence for semantic preprocessing from Korean words and for cross-language parafoveal semantic preview effect. 


\title{
Natural scene perception and eye-tracking
}

\author{
Room: HS 30
}

\section{The influence of image and object features on fixation selection in scene viewing: A generalized linear mixed model approach}

Antje Nuthmann ${ }^{1}$, Wolfgang Einhäuser ${ }^{2}$

1 University of Edinburgh, United Kingdom

2 Philipps-University Marburg, Germany Antje.Nuthmann@ed.ac.uk

Which image characteristics predict where people fixate when encoding natural images? To answer this question, we introduce a new analysis approach utilizing generalized linear mixed models (GLMM). Our method allows for directly describing the relationship between continuous feature value and fixation probability, and for assessing each feature's unique contribution to fixation selection. Key to our approach is an a priori parcellation of the scene. First, we use a grid to obtain homogeneous and exhaustive image coverage. In addition to image features, GLMMs included a predictor that captured the viewing bias towards the centre of the scene. Edge density, clutter, and the number of homogenous segments in a grid cell independently predicted whether image patches were fixated or not; luminance and contrast had no independent effect. Second, we extend the approach to an object-based parcellation to investigate the relative prioritization of objects by their features. Objects that were fixated exactly once during first-pass viewing were not only larger and closer to scene centre, but also visually more salient. Whether or not an object was refixated depended on its size and salience, but not on its distance to image centre. Such prioritization among objects provides evidence for an alternative role of salience.

\section{The independent and causal contributions of chromatic and achromatic features to fixation selection in natural scenes}

\author{
Wolfgang Einhäuser ${ }^{1}$, Antje Nuthmann ${ }^{2}$ \\ 1 Philipps-University Marburg, Germany \\ 2 University of Edinburgh, United Kingdom \\ wet@physik.uni-marburg.de
}

Colour and luminance provide partially redundant information about the content of natural scenes. To assess the independent contribution of colour and luminance features to fixation selection in scenes, we applied a novel method combining a scene-patch analysis with generalized linear mixed models (Nuthmann \& Einhäuser, 2015). Participants freely viewed 108 colour-calibrated natural scenes. Statistical models to predict fixated locations included seven features: luminance, luminance-contrast and edge-density as achromatic features, chroma (saturation), local variation of chroma, and the density of cone-contrast edges as their chromatic counterparts, and distance to image centre as quantification of central bias. To test causality of feature effects, stimuli were displayed in three variants: unmodified, with colour features removed (greyscale), or with luminance features removed (isoluminant). For unmodified stimuli, all tested features except chroma made independent contributions to fixation selection, with the effects of edge densities (chromatic and achromatic) being the most robust across spatial scales. When colour was removed, the effects of achromatic features increased only slightly. In contrast, when luminance was removed, the effects of chromatic features substantially increased. In conclusion, chromatic and achromatic features independently contribute to fixation selection; when both are available, achromatic features overrule some - but not all - of the chromatic effects. 
Talks

\title{
Representing real-world object categories for visuo-motor decisions using category-consistent features \\ Gregory Zelinsky, Justin Maxfield, Chen-Ping Yu \\ Stony Brook University, United States of America \\ gregory.zelinsky@stonybrook.edu
}

How are object categories represented and used during categorical search? A present/absent search for a cued target category revealed that target-fixation-time (guidance) was longest following superordinate-level cues, and decreased with movement down the category hierarchy (subordinate $<$ basic $<$ superordinate). However, time between target fixation and the target judgment (verification) showed the standard basic-level advantage (subordinate $>$ basic $<$ superordinate). To model these behaviors we extracted features from 100 exemplars for each of the 48 subordinate-level categories used in the behavioral experiment and computed bag-of-word histograms for each. Averaging these histograms by category (including 16 basic and 4 superordinates) then reducing their dimensionality gave us category-consistent features (CCFs) -image-based category proto-types. Guidance was modeled by the number of CCFs per category; average CCF number increased with movement down the category hierarchy. Verification was modeled by CCF overlap between a target category and its categorical siblings. CCF overlap was also greatest for subordinates, less for basics, and least for superordinates. The basic-level advantage was modeled by the multiplicative combination of these offsetting trends, with the basic-level enjoying a balance between having sufficient numbers of features (reasonable specificity) and having minimal feature overlap with neighbors (reasonable distinctiveness). Our model makes possible the study of these relationships for real-world object categories.

\section{The influence of a scene preview on eye movement behaviour in natural scenes.}

\author{
Nicola C. Anderson, Mieke Donk, Martijn Meeter \\ Vrije Universiteit Amsterdam, Netherlands \\ n.c.c.anderson@vu.nl
}

\begin{abstract}
A brief preview of a natural scene can provide a substantial amount of information about scene gist, spatial layout, and the semantics of a natural scene. This preview has been shown to improve visual search efficiency even when it is as short as $50 \mathrm{msec}$. The preview advantage is thought to represent the establishment of contextual guidance and to reflect the initial processing taking place during the first fixation on a natural scene. The present work has two aims, first, to investigate whether an initial scene preview affects attentional guidance in natural scenes outside of visual search (i.e., how does the presence of contextual information influence more general eye movement behaviour?) and second, whether it can override the initial tendency for saccades to target regions of high salience. We manipulated the salience distribution across a scene by changing the global contrast. We showed participants a brief real or nonsense preview of the scene and looked at the time-course of attentional guidance. A real preview attenuated early effects of salience, decreased the latency and increased the amplitude of initial saccades into an image. We discuss these findings in terms of theories of stimulus-driven control and contextual guidance in natural scene viewing.
\end{abstract}




\section{Generalized Recurrence Analysis of Eye Movements \\ Walter Bischof ${ }^{1}$, Grayden Solman ${ }^{1}$, Marina Groner ${ }^{2}$ \\ ${ }^{1}$ University of British Columbia, Canada \\ 2 Distance Learning University, Switzerland wfb@ualberta.ca}

There are only a few methods for characterizing the dynamics of eye movements without reference to specific stimuli. Recently, we have introduced recurrence quantification analysis (RQA) as a tool for such characterizations (Anderson, Bischof, Laidlaw, Risko, \& Kingstone, 2013, Behavior Research Methods), and we have shown that it can be used for discriminating between, and assessing the similarity of different eye movements patterns. One drawback of RQA is the use of a spatial threshold for defining recurrence. In the present work, we have generalized RQA to a threshold-free method, which necessitated a re-definition of the usual measures for describing and measuring the recurrence patterns. We present the generalized recurrence analysis, as well as a set of measures for characterizing the recurrence matrices. These include the revisiting of earlier positions and the partial retracing of earlier scanpath segments, as well as their temporal properties. Then we show with several data sets that the generalized RQA is a powerful method for capturing essential characteristics of eye movements and for discriminating normal from abnormal eye movements. 


\title{
Talks
}

\section{Saccades I}

Room: HS 31

\section{The effect of spatial probability and reward on saccadic and manual response times}

\author{
Rosie Clark $^{1}$, Rafal Bogacz ${ }^{2}$, lain D. Gilchrist ${ }^{1}$ \\ ${ }^{1}$ School of Experimental Psychology, University of Bristol, United Kingdom \\ ${ }^{2}$ Nuffield Department of Clinical Neurosciences, University of Oxford, \& Department of Computer \\ Science, University of Bristol, United Kingdom \\ r.clark@bristol.ac.uk
}

The relative probability of stimuli and the reward value associated with them are forms of prior knowledge, influencing our decisions about where to look, and how urgent those decisions are.

Saccadic reaction times are faster to locations that occur more frequently (Carpenter and Williams, 1995), and primate studies have shown mean saccadic latency was shorter in rewarded than in non-rewarded trials (e.g. Bendiksby \& Platt 2006).

In Experiments 1 and 2 we investigated the effect of spatial probability and then spatial reward values on manual response time and saccade latency. We found a significant difference between the manual response times to targets associated with high/low probability \& reward values respectively, but no consistent effect in the saccadic latencies.

In Experiments 3 and 4 we combined reward values and probability. We found that probability dominated the speeding and slowing of responses times; the only significant factor when reward rates were matched or additive (high reward/high probability vs low reward/low probability). Modulations in probability of targets across blocks had a significant effect on the drift bias at fixation.

These results suggest subtle differences in the way that reward and spatial probability have an impact on saccadic and manual responses.

\section{Distribution of attention and parallel saccade programming in antisaccades}

\author{
Anna Klapetek, Heiner Deubel \\ Ludwig-Maximilians-Universität München, Germany \\ anna.klapetek@psy.Imu.de
}

The goal of this study was to investigate the spatiotemporal distribution of attention before pro- and antisaccades. We used a dual task, in which participants made pro- or antisaccades and discriminated the orientation of a visual probe shown during the cueing interval at the saccade goal, the visual cue location (antisaccade condition) or a neutral location.

In line with previous research, we found longer saccade latencies and increased error rates for antisaccades as compared to prosaccades. While pre-saccadic attention in the prosaccade condition was allocated only to the saccade goal, attention in the antisaccade condition was allocated both to the cued location and to the antisaccade goal. The amount of attention at the cue was predictive of the occurrence of errors. Interestingly, most erroneous prosaccades were not recognized by the participants and these unrecognized errors had smaller amplitudes and were corrected faster than recognized errors. The very short corrective saccade latencies provide evidence for parallel saccade programming and were linked to more attention at the antisaccade goal.

In conclusion, our results suggest that goal selection in the antisaccade task is a competitive process, which may result in the parallel programming of an erroneous prosaccade and a subsequent corrective antisaccade. 


\title{
Saccade control and expectation violations in sensorimotor sequences: Evidence for a check-after-surprise mode of attentional selection
}

\author{
Rebecca M. Foerster ${ }^{1,2}$, Werner X. Schneider ${ }^{1,2}$ \\ 1 Neuro-cognitive Psychology, Bielefeld University, Germany \\ 2 'Cognitive Interaction Technology' Cluster of Excellence CITEC, Bielefeld University, Germany \\ rebecca.foerster@uni-bielefeld.de
}

In well-practiced sensorimotor actions, LTM-based expectations control spatial shifts of attention and eye movements to target objects. Violations of learned action-relevant expectations (e.g., location change of target objects) cause a shift to a sensory-based mode of attentional selection (visual search, Foerster \& Schneider, 2015). What happens to selection if non-action-relevant features of target objects are unexpectedly changed in a highly practiced sensorimotor sequence? We investigated this question in a computerized version of the number-connection test. Participants clicked on nine spatially-distributed numbered circles (1-9) in ascending order, while eye movements were recorded. Visual features and locations of target objects stayed constant for 65 acquisition-trials. In 20 consecutive changetrials, number 4 appeared in an action-irrelevant different font. In 15 reversion-trials, number 4 returned to the original font. During the first change trial - the surprise trial -, clicking was slower and larger scanpaths with more fixations were performed. Importantly, the additional fixations were mainly checking fixations on target 4 while acting on later targets - but not fixations immediately made after having fixated the discrepant number 4 for the first time. We suggest that an action-irrelevant change of a target object in a sensorimotor sequence leads to a check-after-surprise mode of attentional selection.

\section{The Geometry of Eye Movement Dynamics}

\author{
Simon Henein, Ilan Vardi \\ EPFL, Neuchatel, Switzerland \\ simon.henein@eplf.ch
}

Eye movements consist of spherical rotations, with orientation generally constrained by Listing's law. Our main result is a complete explicit formulation of ballistic eye movement under the Listing constraint. We present a conceptual framework for eye movement basing the dynamics of Listing motion on the equator of the sphere of unit quaternions, which we call the Listing sphere. Analytical dynamics shows that ballistic Listing motion corresponds to free particle motion on the Listing sphere. Thus, ballistic Listing movement is greatly simplified by transposition to the Listing sphere, where it consists of shortest distance trajectories. This proves that ballistic eye motion consists of constant speed rotation along circles passing through the occipital point. The relevance of the occipital point in eye movement was already noted by Helmholtz, which we explain by the fact that it corresponds to the equator of the Listing sphere. We designed a physical mechanism producing the correspondence between eye movement and particle motion on the Listing sphere. Our straightforward description of ballistic eye motion under the pure Listing kinematic constraint serves as a useful idealized benchmark in the study of actual physiological eye movements, whose orientations are known to deviate slightly from the Listing constraint. 


\title{
Eye movements and reading: Influences of higher cognition
}

Room: HS 32

\section{Mass measurement of fixation behaviours and audience nature in a realistic educational setting}

\author{
Richard Shillcock, Cara Wase \\ The University of Edinburgh, United Kingdom \\ rcs@inf.ed.ac.uk
}

We present a novel extension of a visual inspection task described by Rudoy, Goldman, Shechtman and Zelnik-Manor (2012), to educational settings. A class of 231 students watched a film of a psychology lecture in a standard lecture theatre. The film was frequently briefly interrupted by a $15 \times 15$ grid of dark-on-light letters-and-digits across the whole screen. Students wrote down the letters-and-digits they saw at that moment, revealing the location of their fixation and attention. Students also reported seating position and basic personal data; finally, they completed the TIPI measure of the Big-Five personality domains (Gosling, Rentfrow, \& Swann, 2003). We show (a) heat maps generated from the data qualitatively resemble those created from conventional eye-tracking data; (b) "point and gaze" gestures effectively capture visual attention, and "meaningful" (iconic) gestures and "non-meaningful" (beat) gestures differ in their consequences for audience attention and fixation; (c) measured extraversion predicted chosen distance from the screen, but measured conscientiousness did not. The interaction of fixation behaviours, audience individual differences and lecturing gestures can be efficiently studied in rich realistic educational settings, representing an opportunity for the emerging field of Learning Analytics.

\section{Unraveling the Multimedia Effect in Testing: How Representational Pictures Affect Schoolchildren's Processing of Multiple-Choice Questions}

\author{
Marlit Annalena Lindner, Olaf Köller \\ Leibniz Institute for Science and Mathematics Education (IPN Kiel), Germany \\ mlindner@ipn.uni-kiel.de
}

Recent studies have shown that multiple-choice questions (MCQ) become easier when a representational picture (displaying the same information as the text) is added to the verbal item stem. To shed light on processes underlying this multimedia effect in testing, we recorded eye movements of sixty-two schoolchildren while they were solving naturalscience MCQ either with or without a representational picture to uncover how the picture affects students' focus of attention on MCQ information units (text, picture, question, answer options) and their item-solving behavior. Interestingly, even though pictures constituted an additional item element, our data show that fixation time spent on processing the picture was compensated by saving substantial reading time of the corresponding text. Nevertheless, in text-picture items, students spent more fixation time on studying the item stem as a whole (text + picture), but considerably less time on (especially incorrect) answer options. Overall, our data indicate that pictures helped encoding and representing text information more efficiently and enhanced students' understanding, reflected in longer fixation time on correct compared to incorrect answer options - a pattern that was associated with successful solution behavior in general. Moreover, fine-grained gaze-likelihood analyses reveal how students attended to different MCQ information units over time. 


\title{
Eye movements during the recollection of text information reflect content rather than the text itself
}

\author{
Lund University, Sweden \\ fratraub@googlemail.com
}

Franziska Traub, Roger Johansson, Kenneth Holmqvist

Several studies have reported that spontaneous eye movements occur when visuospatial information is recalled from memory. Such gazes closely reflect the content and spatial relations from the original scene layout (e.g., Johansson et al., 2012). However, when someone has originally read a scene description, the memory of the physical layout of the text itself might compete with the memory of the spatial arrangement of the described scene. The present study was designed to address this fundamental issue by having participants read scene descriptions that where manipulated to be either congruent or incongruent with the spatial layout of the text itself.

28 participants read and recalled three texts: (1) a scene description congruent with the spatial layout of the text; (2) a scene description incongruent with the spatial layout of the text; and (3) a control text without any spatial scene content. Recollection was performed orally while gazing at a blank screen.

Results demonstrate that participant's gaze patterns during recall more closely reflect the spatial layout of the scene than the physical locations of the text. Memory data provide evidence that mental models representing either the situation or the text do not necessarily compete but rather supplement each other.

\section{Comparison of Scanpaths Using Multimatch Algorithm - Influence of Affect, Photo Content, and Individual Differences Maksymilian Bielecki ${ }^{1}$, Domaradzka Ewa ${ }^{2}$, Zakrzewska Marta ${ }^{3}$ \\ 1 University of Social Sciences and Humanities, Poland \\ 2 Polish Academy of Sciences, Institute of Psychology, Poland \\ 3 University of Social Sciences and Humanities, Poland mbielecki@swps.edu.pl}

Purpose: Multimatch (Jarodzka, Holmqvist, \& Nystrom, 2010) is an algorithm allowing to assess several aspects of scanpath similarity. According to our knowledge, our study is the first verifying if Multimatch allows to detect systematic differences related to the content of the photos and their affective properties. Methods: 45 subjects viewed 90 pictures from Nencki Affective Picture Database ( 3 affective and 3 content categories were used). Each photo was presented for 5 seconds. Data were collected using EyeLink 2k eyetracker. Results: For each similarity dimension results were averaged taking into account: within- vs. between subject comparisons, affective characteristics (same vs. different category), and picture content (same vs. different). Multimatch similarity was significantly higher on all five dimensions for within-subjects comparisons. Following our expectations, most of the Multimatch measures showed also greater similarity of pictures from the same affective and content categories. These two variables interacted significantly, as affective similarity was more pronounced for pictures from the same content category. Conclusions: Obtained results indicate that Multimatch is sensitive enough to detect similarity related to affective and content properties of photos, and, therefore, show a promising avenue for further research employing this method. 


\title{
Tuesday, August $18^{\text {th }}, 1.20 \mathrm{pm}-3.00 / 3.20 \mathrm{pm}$
}

\section{Reading: Development and special populations}

\author{
Room: HS 7
}

\section{Young Skilled Deaf Readers Have an Enhanced Perceptual Span in Reading}

\author{
Nathalie N. Belanger, Elizabeth R. Schotter, Keith Rayner \\ University of California, San Diego, United States of America \\ nbelanger@ucsd.edu
}

Skilled hearing readers have been the benchmark population to define typical and successful reading behaviour, but information on skilled deaf readers remains scarce. Accumulating evidence however suggests that reading is qualitatively different in skilled deaf readers relative to skilled hearing readers on several aspects. In non-reading tasks, deaf people have enhanced visual attention to simple stimuli in the parafovea relative to hearing people. Previous research addressed the question as to whether auditory deprivation (deafness) also affects low-level visual processing during reading and compared the perceptual span of deaf signers who were skilled readers to that of skilled hearing readers. Skilled adult deaf readers were found to have a wider perceptual span relative to skilled hearing readers (Bélanger et al., 2012). Recent work with beginning deaf and hearing readers replicate these results. In a moving-window experiment, we show that young deaf readers, matched on reading level to young hearing readers have a wider perceptual span, but also read faster, make longer forward saccades and fewer regressions than their hearing peers (with equal comprehension levels), suggesting that they can pick up word-level information extremely rapidly and efficiently in their fovea and parafovea with no cost to comprehension. 


\title{
Childrens' eye movements during reading: Evidence for deficits in sensorimotor target computation in children with ADHD, but not in children with dyslexia.
}

\author{
André Krügel $^{1}$, Angela Ines Klein ${ }^{2}$, Sarah Risse ${ }^{1}$, Günter Esser ${ }^{1}$, Ralf Engbert ${ }^{1}$ \\ 1 University of Potsdam, Germany \\ 2 Universidade Tecnologica Federal do Parana, Brazil \\ kruegel@uni-potsdam.de
}

Reading is based on eye-movements (saccades) aiming at optimal within-word viewing positions. It is known from skilled adult readers that the sensory computation of target coordinates for saccade planning is based on optimal Bayesian integration of incomplete sensory information and non-sensory prior knowledge (Engbert \& Krügel, 2010; Krügel \& Engbert, 2014). However, very little is known about the low-level sensorimotor processes in beginning readers. Here we present new results from a normal reading experiment with three groups of children: healthy children, children with an attention-deficit/hyperactivity disorder (ADHD) and dyslexic children. Based on a launch-site dependent analysis of saccades' within-word landing positions we demonstrate the characteristic pattern of integrating sensory information and prior knowledge in all three groups. However, children with ADHD show significantly broader landing distributions than the other groups and a weaker launch-site contingent shift of fixation positions within words suggesting non-optimal sensorimotor integration during saccade planning in these children. For the dyslexics, on the other hand, our results show a strong contrast between a largely abnormal global eyemovement behavior (fixation times and skipping/refixation probabilities) but almost unaffected sensorimotor functioning. We will discuss these results against the backdrop of our Bayesian model of saccade planning.

\section{Oculomotor function in children with and without Developmental Coordination Disorder.}

\author{
Emma Sumner, Gustav Kuhn, Elisabeth Hill \\ Goldsmiths, University of London, United Kingdom \\ e.sumner@gold.ac.uk
}

Children with Developmental Coordination Disorder (DCD) present with significant motor difficulties, demonstrating problems with fine and gross motor planning and control. Surprisingly very little research has assessed oculomotor function in these children. The aim of this study was to compare basic oculomotor control in children with and without DCD. Twenty-five children, aged 7-10, with a diagnosis of DCD were matched by age and gender to 25 typically-developing children. Eye movements were recorded as children completed four tasks: fixation, horizontal smooth pursuit, and pro- and anti-saccades. Preliminary analyses reveal that children with DCD demonstrated significant oculomotor deficits compared to their typically developing peers. They had poorer fixation stability and made more drifts away from the visual target. They also made a higher number of saccades during smooth pursuit. Children with DCD had similar pro- and anti-saccade latencies to their peers, but they found the anti-saccade task very difficult, completing less than a quarter of the trials on average due to many anti-saccade errors. The findings are the first demonstration of oculomotor differences in children with DCD. Further examination of oculomotor dysfunction in this population may help to identify neural mechanisms involved in this disorder. 
Talks

\title{
Exploring spontaneous inference generation in children with ASD during reading
}

\author{
Martina Micai ${ }^{1}$, Holly Joseph ${ }^{2}$, Mila Vulchanova ${ }^{3}$, David Saldaña ${ }^{1}$ \\ ${ }^{1}$ University of Seville, Spain \\ 2 Oxford Brookes University, United Kingdom \\ ${ }^{3}$ NTNU, Norway \\ mmicai@us.es
}

Reading strategy in Autism Spectrum Disorder (ASD) while reading texts and answering questions that do, or do not, require an inference to be generated are investigated. Reading comprehension difficulties are well-documented in ASD population, in particular in generating inferences. High functioning children and adolescents with $\operatorname{ASD}(\mathrm{n}=12)$, and typically developing children and adolescents $(n=12)$, matched for no-verbal IQ and reading speed, read five stories, each divided into five paragraphs. Each paragraph required participants to answer a question that could either be inferential or directly mentioned in the text. Expected results will show differences in accuracy rates between the directly mentioned and inferential questions with poorer performance for inferential questions in the ASD group. The differences between groups found in off-line measures will be additionally explored using an EyeLink 1000. In the inferential condition, it is expected that participants with ASD will show a lower tendency to explore the words previously defined as fundamental to question responding, while in the directly mentioned condition, no group differences are expected in eye movement patterns. This study will help investigating the mechanical reading strategy involved in real life poor performance in making inferences in children with ASD.

\section{An incremental boundary study on parafoveal preprocessing in children: Parafoveal masks overestimate the preview benefit.}

\author{
Christina Marx, Stefan Hawelka, Sarah Schuster, Florian Hutzler \\ Centre for Cognitve Neuroscience, Austria \\ christina.marx@sbg.ac.at
}

One crucial factor for fast and fluent reading is parafoveal preprocessing. Over the last decades, most eye-movement studies investigated this process by comparing reading times after preprocessing a valid preview to instances in which parafoveal masks prevented preprocessing (Rayner, 1975). To date, there is a debate whether this investigation overestimates the preview benefit due to interference by the masks (Hutzler et al., 2013). The aim of the current research was to utilize the novel incremental boundary technique as an alternative manipulation for the study of parafoveal preprocessing in children. This technique allowed us to control the salience of the preview by gradually reducing its visual integrity (i.e., displacing a certain amount of black pixels of the preview). Our salience manipulation of valid previews revealed a substantial preview benefit, whereas for the masks, we observed interference. This means that if we had estimated the preview benefit by contrasting the valid previews with the masks, then we would have overestimated the preview benefit substantially. With the novel incremental boundary technique, however, one can achieve an accurate estimate of the preview benefit. 


\title{
Analysis of microsaccades extracted from fixations along sentence reading in young and senior groups
}

\author{
Liliana Raquel Castro ${ }^{1,3}$, Gerardo Fernández ${ }^{2}$, Osvaldo Enrique Agamennoni $i^{3,4}$ \\ ${ }^{1}$ Depto. de Matemática, Universidad Nacional del Sur, Argentina \\ 2 Instituto de Investigaciones en Ingeniería Eléctrica (UNS-CONICET), Argentina \\ ${ }^{3}$ Depto. de Ingeniería Eléctrica y de Computadoras, Universidad Nacional del Sur, Argentina \\ ${ }^{4}$ Comisión de Investigaciones Científicas (CIC), Argentina \\ Icastro@uns.edu.ar
}

Microsaccades shape and statistical properties vary between individual observers. However, there exist some common patterns, specially related with aging. Using the eyetracking technique, in this work we analyze common and different statistical patterns of microsaccades produced during fixations along Spanish binocular sentence reading. For the study, we considered two different groups of healthy people: 40 young adults (mean age $28, \mathrm{SD}=4.2$ years) and 40 old adults (mean age $71, \mathrm{SD}=6.1$ years). We used a sentence corpus composed of 75 regular Spanish sentences that represent a large variety of grammatical structures. We considered all fixations for each eye and each subject along sentence reading and extracted the microsaccadic movements from those fixations. For the analysis, we took into account the whole set of the extracted microsaccades for each eye. From the statistical analysis we conclude that microsaccades average duration is similar on both groups and both eyes while SD average for both vertical and horizontal components of microsaccades are longer on senior adults for each eye. Besides, the number of microsaccades on each fixation is similar for both groups and eyes; instead, the duration of microsaccadic movements for both eyes is longer on senior readers. 
Talks

\title{
Neural mechanisms of saliency, attention and orienting
}

\author{
Room: HS 30
}

\author{
A midbrain saliency map in the primate superior colliculus \\ Brian J. White ${ }^{1}$, David Berg ${ }^{2}$, Laurent Itti ${ }^{3}$, Douglas P. Munoz ${ }^{1}$ \\ ${ }^{1}$ Centre for Neuroscience Studies, Queen's University, Kingston, Ontario, Canada. \\ 2 IBM Research, Almaden, San Jose, California, United States of America \\ ${ }^{3}$ Department of Computer Science, University of Southern California, Los Angeles, United States of \\ America \\ brian.white@queensu.ca
}

Saliency map theory has had a profound impact on how we conceptualize and model the visual attention system. Yet, empirical evidence for the saliency map is based on the use of simple stimuli in restricted viewing conditions, and is only tenuously linked to now wellestablished models. A crucial test of the saliency hypothesis requires generalization to natural dynamic stimuli, which is best achieved with a computational model. We conducted the first test of the saliency hypothesis that compared directly the output of a computational saliency model with the activation patterns of neurons in the superior colliculus, a midbrain structure that predates the evolution of neocortex, and is closely linked to the control of attention/gaze. We describe a compelling set of results in which the model was highly predictive of SC activation. Specifically, SC superficial layers (SCs), whose dominant inputs arise from visual cortex, encoded saliency independent of saccade goals, whereas SC intermediate layers (SCi), whose dominant inputs arise from fronto-parietal cortices, encoded saliency only when the evoking stimulus was the saccade goal. These results implicate the SCs in the role of a bottom-up saliency map, whereas the SCi regulates which salient signals are prioritized for the locus of gaze.

\section{The role of the superior colliculus in the coordination of the pupil orienting response}

\author{
Chin-An Wang, Douglas P. Munoz \\ Centre for Neuroscience Studies, Queen's University, Canada \\ josh.wang@queensu.ca
}

Pupil size, as a component of orienting, changes rapidly in response to local salient events in the environment. A growing body of evidence suggests that the superior colliculus (SC) encodes stimuli based upon saliency to coordinate the orienting response. Although the SC is involved causally in the initiation of saccadic eye movements and attention, its role in coordinating other components of orienting is less understood. Here, we examined how pupil dynamics are modulated by the SC and stimulus saliency. While requiring monkeys to maintain central fixation, we either presented a salient visual, auditory, audiovisual stimulus or delivered weak electrical microstimulation to the intermediate SC layers. Transient pupil dilation was elicited after presentation of salient stimuli, and this dilation was qualitatively similar to that evoked by SC microstimulation. Moreover, the timing and magnitude of evoked pupil responses scaled with the level of stimulus contrast, with significantly faster and larger pupil responses observed for more salient stimuli. The same modulation of stimulus saliency and modality was demonstrated in humans. Together, the results suggest that the transient pupil orienting response is modulated by stimulus saliency, and the SC is a hub in the orienting circuit to coordinate these pupil responses. 


\title{
The role of the frontal eye fields (FEF) in the expression of non-saccadic measures of oculomotor output
}

\author{
Brian Corneil \\ University of Western Ontario, Canada \\ bcorneil@uwo.ca
}

The oculomotor system remains one of the best-understood motor systems in the brain, in no small part because of the ease of measuring discrete large saccadic eye movements in a variety of experimental settings and animal models. However, saccadic eye movements are only one expression of oculomotor output, and converging evidence from anatomical and neurophysiological studies illustrate important differences in the brainstem premotor circuits governing saccadic and non-saccadic expressions of oculomotor output. Because of such differences, developing oculomotor plans can induce changes in pupil dilation, neck or limb muscle recruitment, or fixational microsaccades well in advance of saccadic eye movements. In my talk, I will describe how causal manipulation of FEF activity in non-human primates, via cryogenic inactivation or intracortical microstimulation, influence the elaboration of pupil dilation, microsaccades, and neck muscle activity. These results are largely consistent with the premise that low levels of oculomotor output, here manipulated experimentally via the frontal cortex, can culminate in overt motor output, strengthening the potential value of such non-saccadic measures of oculomotor output in health and disease.

\section{Neuronal response gain modulation around the time of microsaccades Ziad Hafed \\ Tuebingen University, Germany \\ ziad.m.hafed@cin.uni-tuebingen.de}

Microsaccades are modulated in a machine-like manner by stimulus onsets, whether attended or not. Moreover, because of their similarity to larger saccades, microsaccades are governed by peri-movement changes in vision. Given these peri-movement changes, which likely subserve perceptual stability in the face of eye movement, we recently suggested that visual performance changes in covert attentional tasks may be time-locked to microsaccades (Hafed, Neuron, 2013). Consistent with this, here we show in six monkeys and two brain areas implicated in attention (superior colliculus, SC, and frontal eye fields, FEF) that neuronal signatures attributed to covert attention can be observed even without any attentional task. Stimulus-induced SC and FEF responses are either enhanced or suppressed, simply as a function of when/where stimuli appear relative to microsaccades. Given that both response gain enhancement (e.g. attentional capture) and suppression (e.g. inhibition of return) are classic signatures of covert attentional allocation, our results suggest that perimovement changes in vision associated with microsaccades are an integral component of selective processing mechanisms. Not only do our results have strong implications for reinterpreting a large body of literature on covert attention, but they may also fundamentally redefine how we mechanistically view this phenomenon in the first place. 
Talks

\section{Cell and circuit origins of top-down bias in primate prefrontal cortex Thilo Womelsdorf \\ York University, Canada thiwom@yorku.ca}

Neurons in primate prefrontal cortex control the formation of large-scale attention networks, but how the activity of single neurons exerts network control is unknown. This talk outlines possible mechanisms on how rhythmically structured activation of single neurons links to large scale network dynamics.

I will first provide empirical support showing that burst-synchronization serves as potent candidate mechanism to achieve network control during attentional states. Prefrontal burst firing of single neurons increases during attentional states, synchronizes long-range those prefrontal and anterior cingulate cortices that also functionally interact during controlled behavior, and is subserved by specific cell-types - with interneuron bursts likely triggering remote LFP coherence at beta frequencies and pyramidal cell bursts reflecting most likely coherent dendritic-input.

This example-case of attention-specific burst synchronization gives rise to a Dynamic Circuit Motifs hypothesis that predicts a unique linkage of (1) the (burst-) synchronized activation state, (2) the underlying sturctural components (cell types and dendritic currents), and (3) the generic computational function implemented by the activated motif (context-dependent gating). The talk discuss the challenges and merits of a Dynamic Circuit Motifs perspective to advance our understanding of attention. 


\title{
Eye movements: Neurophysiology and neuropsychology
}

\author{
Room: HS 31
}

\section{Functional connectivity changes after a frontal cortex lesion in a non-human primate}

\author{
Ramina Adam ${ }^{1}$, Kevin Johnston ${ }^{1}$, R. Matthew Hutchison ${ }^{2}$, Stefan Everling ${ }^{1}$ \\ 1 The University of Western Ontario, London, ON, Canada \\ 2 Harvard University, Cambridge, MA, United States of America \\ radam4@uwo.ca
}

Unilateral spatial neglect is an attention deficit commonly seen following right hemispheric stroke. It is characterized by an inability to attend to contralesional stimuli, resulting in a saccade choice bias to the ipsilesional hemifield. Here, we aim to correlate the behavioural recovery of saccade choice bias in spatial neglect with changes in whole-brain functional connectivity (FC). We created a macaque model of ischemic stroke using endothelin-1 injections in the right dorsolateral prefrontal cortex and frontal eye field as verified by anatomical magnetic resonance imaging (MRI). Following stroke, the animal exhibited both a profound ipsilesional saccade choice bias and increased contralesional saccadic reaction times. Resting-state functional MRI (rs-fMRI) scans obtained one week following stroke showed reduced frontoparietal FC in the ipsilesional hemisphere and some increased FC between the contralesional frontal cortex and contralesional and ipsilesional posterior parietal cortex. Data from daily behavioural eye tracking and biweekly rs-fMRI will be collected for another five months or until behavioural performance recovers to pre-stroke levels. Results will reveal how changes in whole-brain FC following stroke are related to the behavioural recovery of saccade choice bias in spatial neglect.

\section{Removal of an epileptically compromised frontal eye fields restores antisaccade behavior}

\author{
Stefan Van der Stigchel ${ }^{1}$, Zachary Freudenburg ${ }^{2}$, Mariska Vansteensel ${ }^{2}$, Chris Dijkerman ${ }^{1}$, \\ Nick Ramsey², Frans Leijten ${ }^{2}$ \\ ${ }^{1}$ Experimental Psychology, Helmholtz Institute, Utrecht University, Netherlands \\ 2 Department of Neurology and Neurosurgery, Brain Center Rudolf Magnus, University Medical Center \\ Utrecht, Netherlands \\ s.vanderstigchel@uu.nl
}

The frontal eye fields (FEF) are heavily involved in resolving oculomotor competition. Here we show the plasticity of the oculomotor cortex in a young epileptic patient in whom the seizure focus included the anatomical location of the left FEF. Before surgery, antisaccade performance showed that the patient had difficulty initiating voluntary saccades to the visual field contralateral to the seizure focus $(26.0 \%$ vs. $7.9 \%$ errors; $p<.04)$. Functional neuroimaging before surgery revealed that the peak of neural activity during the antisaccade task was remote from the anatomical location of the FEF. Electrical subdural stimulation applied to the left FEF was unable to induce increased directional errors during the antisaccade task. Two days after removal of the seizure focus, the patient still showed a behavioral imbalance on the antisaccade task. Two months after surgery, however, the seizures had disappeared and antisaccade performance was the same for both visual fields $(10.8 \%$ vs. $11.1 \%$ errors; n.s.). We hypothesize that a seizure focus at the anatomical location of the FEF can induce a dysfunction in oculomotor selection processes which is resolved after removal of the FEF, perhaps due other areas in the oculomotor cortex taking over the functioning of the FEF. 
Talks

\author{
Patients with parietal lesions encode corollary discharges for \\ endogenously driven bilateral saccades \\ Kate Rath-Wilson, Daniel Guitton \\ Montreal Neurological Institute, Canada \\ kate.rath-wilson@mail.mcgill.ca
}

In performing the classic double step task, parietal lesion patients have been shown to fail at generating an accurate second saccade when the first one is directed contralesionally, but not ipsilesionally, suggesting an impairment in contralesional corollary discharges (CD). Here, we show that this conclusion is oversimplified. We tested five patients with parietal lobe lesions on a classic double step task; when patients were given sufficient time and corrective saccades were evaluated, some patients showed evidence of $\mathrm{CD}$ for first saccades in either direction (two of five patients). The other patients failed, due mostly to their inability to break fixation. We hypothesize that this difficulty relates to well-described attentional impairments in parietal lesion patients. We then tested six patients on a modified version of the double step task, designed to mitigate some of the common attentional deficits associated with parietal lesions by presenting a single target for a longer period of time, and by asking the patients to make a first endogenous saccade of self-determined amplitude before making a second saccade to the remembered location of the previously flashed target. Every parietal patient tested showed evidence of $\mathrm{CD}$ for endogenous first saccades in both directions (six of six patients).

\title{
Crude calibration is sufficient for blind users to extend the functionality of a retinal prosthesis
}

\author{
Avi Caspi ${ }^{1,2}$, Arup Roy ${ }^{2}$, Robert Greenberg ${ }^{2}$, Avinoam B. Safran ${ }^{3}$, Jose A. Sahel ${ }^{3}$ \\ 1 Jerusalem College of Technology - Lev Academic Center, Israel \\ 2 Second Sight Medical Products, Inc. Sylmar, California, United States of America \\ ${ }^{3}$ Paris-6 University and Institut de la Vision, Paris, France \\ avi.caspi@gmail.com
}

Eye tracking of a blind person presents several challenges, specifically the difficulty of calibrating due to a lack of visual feedback. Yet eye tracking is desirable for users of retinal prostheses. In the Argus II Retinal Prosthesis System, which is approved in USA and in Europe, stimulation at retinotopic coordinates is based on real-time images acquired by a camera mounted on the glasses and users scan the scene with head movements. Integration of an eye tracking would enable more naturally control the region of interest of the image that will be mapped to the prosthesis.

We demonstrated that blind patients implanted with the Argus II retinal prosthesis can scan the scene with eye movements using crude calibration of the eye tracker along the horizontal axis. Patients were instructed to look to the right and the left, and a video-based eye tracker was calibrated to match a response of minus and plus 45 degrees respectively at these limits with liner interpolation in-between. Blind subjects with the Argus II were able to scan horizontally using their eye movements. Subjectively, patients reported that eye scanning is easier as his body can be used as a frame of reference when using eye scanning. 


\title{
The effect of partial versus total field removal on search strategies in simulated hemianopia
}

\author{
Anna D. F. Nowakowska, Alasdair D. F. Clarke, Amelia R. Hunt, Arash Sahraie \\ University of Aberdeen, United Kingdom \\ r02al13@abdn.ac.uk
}

Patients with hemianopia tend to start searching a visual display from their intact visual field, causing a larger proportion of the search array to fall within the damaged field. Rehabilitation programs often train patients to make large eye movements into their damaged field to increase the visible area. We show that the effectiveness of this strategy depends on where and what kind of information is present in both the damaged and intact field. Healthy observers searched for an angry face among neutral faces with half of their visual field degraded or removed. Observers made more saccades towards the sighted field over the blind, and the proportion of saccades directed towards the blind field increased with the amount of information available. However, searching the initially sighted field first was not associated with slower search. Shifting the location of the initial fixation point into the "blind" side, making the search array fully visible at the start of the trial, also did not improve search overall: The target was found faster when it was on the damaged side, but slower when it was on the sighted side, negating the benefit of starting search on the "blind" side. 


\title{
Reading: Dyslexia and aphasia
}

\author{
Room: HS 32
}

\section{Phonological processing during silent reading in readers with dyslexia}

\author{
Jonathan H. Dickins, Hazel I. Blythe, Colin R. Kennedy, Simon P. Liversedge \\ University of Southampton, United Kingdom \\ J.Dickins@soton.ac.uk
}

A deficit in phonological processing has been implicated as a causal factor in developmental dyslexia (Vellutino, Fletcher, Scanlon, \& Snowling, 2004). Eye movement research has demonstrated that skilled adult readers process phonological information, both foveally and parafoveally, during lexical identification in silent sentence reading (Rayner, Pollatsek, \& Binder, 1998; Pollatsek, Lesch, Morris, \& Rayner, 1992). It is currently unclear whether such effects occur in younger readers (with or without dyslexia). Four participant groups were recruited: older (16-18 years) and younger (13-15 years) teenagers, with and without dyslexia, allowing chronological age and reading ability matched comparisons. Eye movements were recorded as they read sentences containing three types of target words: correct words (e.g., church); pseudohomophones (e.g., cherch); and orthographic controls (e.g., charch). Visual similarity of the pseudohomophones and control words to the correct target was manipulated by varying the number of letter changes for each triplet of targets. In a second experiment, the same target words were inserted into new sentences and presented using the boundary paradigm (Rayner, 1975). Sensitivity to our manipulations and general patterns of eye movement behaviour varied between groups, and findings are discussed in the context of theoretical standpoints on phonological processing in reading (Leinenger, 2014).

\section{Dyslexic adult readers show transposed-letter effects in reading Rhiannon S. Barrington ${ }^{1}$, Denis Drieghe ${ }^{2}$, Julie A. Kirkby ${ }^{1}$ \\ ${ }^{1}$ Bournemouth University, United Kingdom \\ 2 University of Southampton, United Kingdom rbarrington@bournemouth.ac.uk}

Target words (e.g. judge) preceded by a parafoveal preview in which letters are transposed (e.g. jugde) receive shorter fixation times compared to when letters in the preview are substituted (e.g. junpe). This transposed-letter effect in skilled adult reading has been taken as evidence for a language processing system that extracts letter identity information independent to absolute letter position (Johnson, Perea, \& Rayner, 2007). Under specification of letter position encoding, however, has been causally linked to developmental dyslexia (e.g. Friedmann \& Rahamim, 2007). We directly examined this by recording eye movements from both skilled adult readers and adults with dyslexia. A boundary paradigm was used as single line sentences were silently read and parafoveal previews were either identical to the target (e.g. before), a transposed-letter non-word neighbour (e.g. ebfore), or a substitutedletter non-word (e.g. tcfore). The results showed disruption in reading for previews with transposed letters and substituted letters compared to baseline identical previews with less disruption from transposed letter previews compared to substituted letter previews. This pattern of effects held for both groups. The typical transposition effect observed in adult dyslexic readers has important implications for the causal role of letter position encoding in dyslexia. 


\title{
Benefits of Increased Character and Word Spacing for Developing Chinese Readers with Dyslexia: Evidence from Eye Movements
}

\author{
Jingxin Wang ${ }^{1}$, Sha $\mathrm{Li}^{1}$, Lin $\mathrm{Li}^{1}$, Fang $\mathrm{Xie}^{1}$, Min Chang ${ }^{1}$, Victoria A. McGowan ${ }^{2}$, Kevin B. \\ Paterson ${ }^{2}$ \\ ${ }^{1}$ Academy of Psychology and Behavior, Tianjin Normal University, People's Republic of China \\ ${ }^{2}$ College of Medicine, Biological Sciences and Psychology, University of Leicester, United Kingdom \\ wjxpsy@126.com
}

Text in Chinese is usually a sequence of box-like pictorial symbols (characters). Characters often combine with adjacent characters to form words but the boundaries between words are not demarcated using spaces or other visual cues. Adding spaces between words can nevertheless facilitate the performance of typically-developing Chinese readers and secondlanguage learners. But the potential benefits of adding spaces to text for readers with dyslexia are unknown. Eye movements provide a detailed measure of reading performance. Accordingly, the present experiment used eye movement measures to reveal how adding spaces between characters affects the performance of atypically-developing (dyslexic) readers and age- and ability-matched groups of typically-developing readers. Text was presented normally, or with spaces between all characters, or between character pairs that combined to form either words or non-words. Compared to normal text, increased spacing showed no overall facilitation for typically-developing readers, and incurred a cost for all readers in the non-word condition. But clear facilitation was observed for atypicallydeveloping readers when spaces were added between characters or words. The results indicate, for the first time, that increased character spacing can benefit the reading performance of Chinese readers with developmental dyslexia.

\section{Mindless reading in children with attention deficit, dyslexia and the comorbid condition}

\author{
Thomas Günther ${ }^{1}$, Katharina Peters ${ }^{1}$, Wolfgang Scharke ${ }^{1}$, Ralph Radach ${ }^{2}$ \\ ${ }^{1}$ Child Neuropsychology Section, Department of Child and Adolescent Psychiatry, Medical Faculty, \\ RWTH Aachen University, Germany \\ 2 Department of Psychology, University of Wuppertal, Germany \\ tguenther@ukaachen.de
}

Aim of this study was to examine the influence of attention deficits (AD) on dyslexia (DY). Children in 3rd and 4th grade participated: 24 with DY, 32 with AD, 25 with DY and AD (DYAD) and 29 normal controls (NC). All children read 36 age-appropriate sentences in the first and the same sentences in a second condition, wherein each letter was replaced by a Landolt ring with the same size (mindless reading).

Spatial and temporal eye movement parameters in reading were negatively influenced by an AD, whereas children with DYAD and DY were comparable impaired on nearly all eye movement parameters. As dyslexia is described as a primarily linguistic disorder it is surprising that the eye movements of both dyslexic groups (DYAD and DY) were not comparable with those of NC in the mindless reading task.

The divergent eye movements of children with dyslexia on the mindless reading paradigm could not explained by attention deficits. Future studies have to clarify whether the impairments in mindless reading could be an explaining factor for reading problems (e.g. occulomotor deficits) or that it is a consequence due to a different automatized reading behavior that is generalized to the mindless reading task. 
Talks

\title{
Meaning-structure probabilities influence comprehension of mild garden path sentences in aphasia
}

\author{
Anneline Huck ${ }^{1}$, Madeline Cruice ${ }^{1}$, Robin Thompson ${ }^{2}$, Penny Roy ${ }^{1}$, Jane Marshall ${ }^{1}$
}

${ }^{1}$ School of Health Sciences, City University London, United Kingdom

${ }^{2}$ School of Psychology, University of Birmingham, United Kingdom

Anneline.Huck.1@city.ac.uk

The same verb can combine with different sentence structures, depending on subtle properties of its meaning. The verb acknowledge, for example, takes a direct object (DO) when it means "recognize", but prefers a sentence complement (SC) when it means "admit". We explored whether people who had a stroke and are diagnosed with the language impairment aphasia can exploit these meaning-structure probabilities during the reading of temporarily ambiguous sentences. Using eye-tracking, people with mild to moderate aphasia (PWA) and controls read target sentences with a SC structure (e.g. He acknowledged (that) his friends would probably help him a lot). Without 'that' the sentence was temporarily ambiguous, because 'his friends' could be interpreted as the DO. The addition of 'that' removed the ambiguity. A prime sentence created a DO- or SC-biased sense of the verb. PWA were less accurate and had longer fixation durations than the controls. However, both groups showed shorter fixation durations when the prime sentence was SC-biasing as compared to DO-biasing, and when the sentence was temporarily non-ambiguous as compared to ambiguous. This indicates that PWA may retain meaning-structure probabilities in their language system which they can draw on if faced with the interpretation of syntactically complex sentences. 


\title{
Tuesday, August $18^{\text {th }}, 5.00 \mathrm{pm}-6.40 \mathrm{pm}$
}

\section{Reading: Influences of context I}

\author{
Room: HS 7
}

\section{Spontaneous transfer of syntax in natural reading}

Awel Hydref Vaughan-Evans ${ }^{1}$, Simon P. Liversedge ${ }^{2}$, Guillaume Thierry ${ }^{1}$, Manon Jones ${ }^{1}$

1 Bangor University, United Kingdom

2 University of Southampton, United Kingdon

pspea5@bangor.ac.uk

In a recent ERP study, we demonstrated that Welsh-English bilinguals anomalously transfer syntactic knowledge between languages (Vaughan-Evans et al., 2014, Journal of Neuroscience). In Welsh, a morphosyntactic rule called soft mutation alters the initial consonant of nouns when presented in specific grammatical contexts. We showed that English nonwords conforming to Welsh mutation rules (e.g., ' brince' derived from 'prince'), were more acceptable when presented in a syntactic context that would elicit a mutation in Welsh. In this eye-tracking study, we examined the effect in more naturalistic reading conditions. Thus, the target word 'brince' could be viewed in the parafovea, but turned to 'prince' upon fixation. The factor 'word mutation' ('no mutation': prince vs. 'correct mutation': brince vs 'aberrant mutation': grince; the latter comprising an initial phoneme substitution that violated the rule), was crossed with the factor 'sentence type' ('mutation sentence' vs 'no mutation sentence'). Preview of a correctly mutated word (brince) elicited shorter processing times on word n-1 but only when presented in a mutation sentence. A similar effect did not occur for aberrant mutations (grince). Our findings support the hypothesis that bilinguals spontaneously and subconsciously transfer syntactic rules across languages, even in a natural reading context.

\section{No word is an island: How eye-tracking helps us to understand the processing advantage when native speakers read familiar sequences}

\author{
Gareth T. Carrol, Kathy Conklin \\ University of Nottingham, United Kingdom \\ gareth.carrol@nottingham.ac.uk
}

Formulaic language (e.g. idioms, collocations) is processed in a qualitatively different and quantitatively faster way than "novel" language. We explore this for a range of formulaic types in two eye-tracking experiments.

First, do we see facilitation of known sequences when no biasing context exists to aid interpretation? We presented formulaic items vs control phrases in a natural reading study. Our results showed a consistent advantage for all formulaic types. This was true for idioms (spill the beans vs. drop the beans), binomials (e.g. king and queen vs. prince and queen) and collocations (abject poverty vs. awful poverty). Crucially, eye-tracking allows us to explore phrase and word-level reading patterns to discern the locus of the advantage.

Second, we examine component words used in non-formulaic configurations, again using eye-tracking to explore lexical processing in early (skipping rates, first fixation times) and late measures (total reading times, regression durations). Results point to facilitation in idioms, and an effect of semantic relatedness but not formulaicity in other sequences.

We discuss both studies in terms of the implications for the mental lexicon, and specifically how eye-tracking can help us to understand the processes at work when native speakers encounter single words and lexicalised multiword combinations. 
Talks

\title{
The effect of hyphenation at syllable boundaries in early Finnish reading instruction
}

\author{
Raymond Bertram, Tuomo Häikiö, Jukka Hyönä \\ University of Turku, Finland \\ rayber@utu.fi
}

Finnish ABC books present words with hyphens inserted at syllable boundaries. Syllabification by hyphens is abandoned in the 2nd grade for bisyllabic words, but continues for words with three or more syllables. The current eye movement study investigated how hyphenation of bisyllabic (kah-vi 'coffee') and multisyllabic words (haa-ruk-ka 'fork', ap-pel-sii-ni 'orange') affects eye movement behavior of 1st and 2nd graders. The results demonstrated that hyphenation increases the number of fixations as well as gaze and selective regression path durations. However, the disruption effects were modulated by readers' proficiency and number of syllables. For short bisyllabic words there was only a disruption effect of hyphenation in gaze duration for 2nd graders, whereas for long multisyllabic words disruption effects were obtained in gaze and selective regression path duration for both 1st and 2nd graders. This implies that hyphenated multisyllabic words were difficult to process when first encountered, but also hard to integrate with prior sentence context. Taken together the results indicate that hyphenation at syllable boundaries attracts attention to the initial syllable and thereby delays processing of subsequent syllables or the whole word directly. We discuss possible implications of our findings for practice in early Finnish reading instruction.

\section{Does text contrast mediate binocular advantages in reading? Stephanie Jainta ${ }^{1}$, Mirela Nikolova ${ }^{2}$, Simon P. Liversedge ${ }^{2}$ \\ ${ }^{1}$ IfADo - Leibniz-Institut für Arbeitsforschung, Germany \\ 2 Psychology, University of Southampton, United Kingdom jainta@ifado.de}

Binocular reading comes with an advantage: reading speed is high and word frequency effects emerge during fixations, which is not the case for monocular reading. A potential contributor to this benefit is the reduced contrast in monocular reading: reduced text contrasts in binocular reading are known to slow down reading and word identification. To investigate whether contrast reduction mediates the binocular advantage, we tested 64 adults in 3 experiments and first replicated increased reading time and nullified frequency effects for monocular reading. Assuming, further, that signal-to-noise discrimination forms a basis for feature detection, one generally needs a 1.4 times higher contrast $(\sqrt{ } 2)$ to detect a monocular compared to a binocular text. Thus, we reduced contrast for binocular sentences to $70 \%$ (Weber-contrast), which resembles monocular conditions (70\% equals $100 \%$ divided by 1.4 ), and predicted effects as in monocular reading. Instead, reading speed and word identification remained unchanged. Finally, we substantially reduced contrast to $40 \%$ and replicated slowed reading and word identification, but still obtained a clear word frequency effect for low-contrast text (Experiment 3). A reasonable conclusion, therefore, is that a reduction in contrast is not the (primary) factor that mediates less efficient monocular lexical processing. 


\title{
Do resource constraints affect lexical processing? Evidence from eye movements
}

\author{
Mallorie Leinenger, Mark Myslín, Keith Rayner, Roger Levy \\ University of California, San Diego, United States of America \\ mleineng@ucsd.edu
}

Previous research has found evidence for "digging-in effects" during syntactic ambiguity resolution-i.e., more costly ambiguity resolution as the parser becomes more committed to the wrong syntactic choice (Levy et al., 2009; Tabor \& Hutchins, 2004), suggesting that syntactic ambiguity resolution is subject to resource constraints. However, previous research investigating resource constraints on lexical ambiguity resolution has yielded conflicting results. In two experiments, we recorded eye-movements during reading to test for evidence of digging-in effects in lexical ambiguity resolution; asking if a reader would become more committed to a given interpretation of a homograph with more intervening material before disambiguation. We embedded moderately biased homographs in sentences with neutral prior context and either long or short regions of text before disambiguation to the dominant or subordinate interpretation. We found no evidence for digging-in effects, as the length of intervening material had no effect on ease of disambiguation. Instead, we found only a main effect of meaning at disambiguation, such that readers made more regressions back to and spent longer re-reading the homograph following subordinate disambiguation, suggesting that disambiguating to the subordinate meaning was more difficult. Our results suggest that lexical ambiguities are resolved rapidly, even without prior contextual disambiguating information. 


\title{
Reading: Influences of valence, emotion, and negation
}

\author{
Room: HS 30
}

\section{Valence, arousal and credibility of an argument influence eye movements during sentence reading}

\author{
Johanna K. Kaakinen, Suvi Peltoniemi \\ University of Turku, Finland \\ johkaa@utu.fi
}

Forty-five participants holding varying attitudes towards immigration read 39 immigrationrelated arguments while their eye movements were recorded. After reading each argument, participants rated the credibility and valence of the argument and the arousal induced by the sentence. Linear mixed effects models were used to examine the relationship between sentence ratings and word level eye movement measures. The results showed that first fixation duration was not influenced by credibility, valence or arousal induced by the sentence. Valence and arousal had significant effects on gaze duration, such that negative valence and high arousal slowed down reading of the words towards the end of the sentence. Low argument credibility increased regression path duration for words in the end of the sentence. In accordance with some recent studies (Knickerbocker et al., 2014), the present results demonstrate that the reader's subjective emotional experience plays a crucial role in eye movement guidance during sentence reading.

\section{Examining the timecourse of the emotional impact of verbal irony: Evidence from eye movements during reading Ruth Filik ${ }^{1}$, Hartmut Leuthold ${ }^{2}$ \\ 1 University of Nottingham, United Kingdom \\ 2 University of Tübingen, Germany ruth.filik@nottingham.ac.uk}

It is currently unclear whether the use of verbal irony serves to enhance or mute the emotional force of an utterance, compared to literal language. To address this question, we used eye-tracking while reading to investigate the moment-to-moment processing of a character's emotional response to ironic vs. literal criticism. In Experiment 1, we monitored participants' eye movements as they read stories describing a character being upset following criticism from another character. Results showed that participants initially more easily integrated a hurt response following ironic criticism; in contrast, later eye movement measures indicated that readers ultimately found it easier to integrate a hurt response following literal criticism. In Experiment 2, characters were instead described as having an amused response, to which participants ultimately showed more integration difficulty following literal criticism. From this, we propose that whilst readers may initially expect a character to be more hurt by ironic than literal criticism, they ultimately rationalise ironic criticism as being less hurtful, perhaps because it can be perceived as being amusing. 


\title{
The role of working memory and emphasizing skills in the processing of written sarcasm
}

\author{
Henri Olkoniemi, Johanna K. Kaakinen \\ University of Turku, Finland \\ hoolko@utu.fi
}

Recent eye-movement studies have shown that the processing of sarcastic (in comparison to literal) utterances is characterized by increased looking back in text. These results indicate that comprehension of sarcasm is an effortful process. Moreover, studies have shown that there are individual differences in the processing of sarcasm. For example, working memory capacity and sensitivity to the emotional state of the speaker have been shown to play a role in sarcasm comprehension. In the present study 60 participants read sarcastic and literal utterances embedded in short texts while their eye-movements were recorded. Texts were composed of 1-2 context sentences and a dialogue with 2 lines. The context sentences were presented either before or after the dialogue. In the dialogue, the first utterance was either sarcastic or literal. Individual differences in working memory capacity (WMC) and emphasizing skill were measured. Sentence-level measures were computed from the eyemovement data. In line with previous findings, the results showed that sarcasm produces mainly delayed effects in the eye-movement records. Emphasizing skill and the location of context (before/after dialogue) had an effect in the processing of sarcasm. However, no effects were observed for WMC. The results have implications for current theories of sarcasm comprehension.

\section{The role of contextual factors in written sarcasm comprehension}

\author{
Alexandra Turcan, Hannah Howman, Ruth Filik \\ University of Nottingham, United Kingdom \\ Ipxat3@nottingham.ac.uk
}

Contemporary accounts of sarcasm comprehension make different predictions regarding whether contextual factors can influence the initial processing of a sarcastic comment. Specifically, modular accounts predict that contextual factors cannot influence initial processing, whereas interactive accounts predict that they can. Thus, in order to distinguish the predictions of these accounts, three eye-tracking studies were designed to systematically investigate the influence of contextual factors on sarcasm comprehension. Each study required participants to read short stories that could end in either a literal or a sarcastic target remark. In Experiment 1, the context either did or did not create an "ironic environment" (Utsumi, 2000). In Experiment 2, an expectation for sarcasm was created by introducing a sarcastic character. In Experiment 3, the target remark was an echo of a previous contextual utterance (Wilson \& Sperber, 1981). Results indicated that although the creation of an ironic environment did not aid sarcasm comprehension, introducing a sarcastic character into the story did - when a character had previously uttered a sarcastic remark in the context, a sarcastic target remark was read faster than a literal one in the initial stages of processing. Experiment 3 is currently in progress. Implications for theories of sarcasm comprehension will be discussed. 
Talks

\title{
Processing negated bounded and unbounded expressions during reading: An eye movement investigation
}

\author{
Lewis T. Jayes ${ }^{1}$, Hazel I. Blythe ${ }^{1}$, Kevin B. Paterson ${ }^{2}$, Simon P. Liversedge ${ }^{1}$ \\ ${ }^{1}$ University of Southampton, United Kingdom \\ 2 University of Leicester, United Kingdom \\ lj7g09@soton.ac.uk
}

The boundedness hypothesis suggests negated mental representations are sensitive to the semantic configuration of the negated entity. Bounded entities are categorical; only one state is possible so, when negated, a bounded entity must be interpreted as its antonym (not dead=alive). Unbounded entities possess a scalar ontology, with many states in between the antonyms; when negated they are ambiguous and can refer to multiple states (not wide $\neq$ narrow). To investigate online interpretation of bounded and unbounded negations, participants read passages with two statements from different characters describing a bounded/unbounded entity. The two accounts were either a repetition (not dead-not dead), a contradiction (not dead-not alive) or were complementary (alive-not dead). We found differential eye movement patterns for the contradictory and complementary (relative to control) conditions as a result of boundedness. There are two main conclusions: (1) the unbounded contradictory condition disrupted reading to a lesser degree than their bounded equivalents; (2) unbounded complementary passages were more difficult to interpret than their bounded equivalents, evidenced by increased disruption to reading. This is the first demonstration of boundedness effects in reading. Their interactive influence with negation suggests representations of bounded entities are categorically discrete, whilst representations of unbounded entities are continuous. 


\title{
Eye movements: Neurophysiology and EEG
}

\author{
Room: HS 31
}

\section{Saccadic Eye Movements and Neural Correlates Involved during Letter and Object Naming Speed Tasks}

\author{
Noor Z. Al Dahhan, Donald C. Brien, John R. Kirby, Douglas P. Munoz \\ Queen's University, Canada \\ noor.aldahhan@queensu.ca
}

Functional magnetic resonance imaging (fMRI) was used to investigate the neural substrates and cognitive processes underlying performance during letter and object naming speed (NS) tasks. We used three methods to examine task performance:(a) changing stimulus composition to emphasize phonological and/or visual aspects; (b) decomposing NS times into pause and articulation components; and (c) analyzing eye movements and brain activation involved in a NS task. 20 healthy young adults (ages 21 - 26 years) were recruited. We employed a block design consisting of a letter NS task and three variants that were either phonologically and/or visually confusing (Compton, 2003); and an object NS task with a variant in which the object names rhymed with one another, while subjects' eye movements and articulations were recorded. Behavioural results indicate that NS performance, NS components, and eye movements were negatively affected when the letters were visually similar compared to when they were phonologically similar. fMRI results indicate significant activation during both letter and object NS tasks in regions consistent with areas involved in the reading network and in tasks that require eye movement control and attention in typical adult readers, such as the temporo-parietal area, inferior frontal cortex, and the ventral visual stream. 
Talks

\title{
Expecting a Surprise? EEG Signal Dynamics in Unrestricted Natural Visual Search
}

\author{
Alexander Varatharajah ${ }^{1,4}$, Juan E. Kamienkowski ${ }^{2,3}$, Lisandro N. Kaunitz ${ }^{1,5}$, Mariano \\ Sigman $^{2}$, Rodrigo Quian Quiroga ${ }^{1,4}$, Matias J. Ison ${ }^{1,4}$ \\ 1 Department of Engineering, University of Leicester, LE1 7RH, United Kingdom \\ 2 Laboratorio de Neurociencia Integrativa, Departamento de Física, FCEyN UBA and IFIBA, Conicet, \\ Pabellón 1, Ciudad Universitaria, 1428 Ciudad Autónoma de Buenos Aires, Argentina \\ 3 Universidad Diego Portales, Santiago, Chile \\ ${ }^{4}$ Centre for Systems Neuroscience, University of Leicester, LE1 7QR, United Kingdom \\ 5 T-lab, School of Psychological Sciences Faculty of Biomedical and Psychological Sciences, Monash \\ University 770 Blackburn Road, Clayton, VIC, 3168, Australia \\ av149@le.ac.uk
}

Visual search is a skill that if not mastered, would leave you considerably unfit for Darwin's idea of survival. Prior knowledge of the cognitive processes involved in visual search stemmed from modeling and behavioral studies collecting subjects' reaction times, their eye movements, or EEG target signatures under fixed-gaze. Recent co-registration of EEG and Eye-Tracking has further enhanced this understanding by allowing brain responses to be seen in the form of fixation-Event Related Potentials (fERPs). To date, only a handful of studies have investigated brain correlates of visual search paradigms involving unrestricted eye-movements. And as of yet there is no constructed standard to form the basis of analysis of free viewing experiments utilizing EEG. In this research, subjects search for targets in natural images, resembling "Where's Waldo?" Results strengthen our previous findings in a visual search task (Kaunitz et al., NeuroImage 2014); showing early and late target detection effects. We have found local and global fERPs driving characteristics that we related to classical concepts of expectancy and surprise. We also show the potential pitfalls from commonly used techniques, requesting the call for an fERP analysis standard.

\section{Simultaneous processing of EEG and eye movements in free viewing behavior}

\author{
Andrey R. Nikolaev, Cees van Leeuwen \\ KU Leuven - University of Leuven, Belgium \\ Andrey.Nikolaev@ppw.kuleuven.be
}

For investigating a wide range of perceptual and cognitive processes a promising alternative to the traditional stimulus-response paradigm seems to be available, in the co-registration of electrical brain activity (EEG) and eye movements in natural visual environments. Technical problems associated with co-registration, however, have limited this approach, in particular as a consequence of overlapping effects on EEG of subsequent eye movements. Using data collected during free visual exploration of natural scenes, we illustrate the main properties of EEG activity around a saccade, consider the difference of the EEG activity time-locked to saccade and fixation onsets, and discuss possible confoundings between cognitive and eye movement factors during co-registration. We elucidate these points with the time-frequency analysis of EEG activity in fixation intervals, which includes measurement of EEG power and inter-trial coherence. We describe a range of research questions that can be approached by co-registration, the requirements for experimentation, and methodological solutions for simultaneous processing of EEG and eye movements. Our contribution is aimed at vision scientists and psychologists who investigate free viewing behavior and who plan to consider in their research neurophysiological correlates of the corresponding brain processes. 


\title{
The effect of secondary cognitive task demand on fixation event related potentials (fERPs) during a hazard perception task
}

\author{
Steven W. Savage, Benjamin W. Tatler, Douglas D. Potter \\ University of Dundee, United Kingdom \\ swsavage@dundee.ac.uk
}

The aim of our research was to determine to what extent secondary cognitive task demand interferes with visual processing in a video based search task, namely driving hazard perception.

As monitoring electrophysiological activity during fixations is thought to provide insight into the self-paced acquisition of perceptual information within visual scenes, we compared average differences in fERPs between high and low cognitive load conditions. When secondary cognitive load was high, activity of fERPs were more negative at occipital, parietal and temporal sites and more positive at frontal-central electrodes $50-150 \mathrm{~ms}$ following fixation onsets.

More negative fERPs at occipital sites may suggest that the incoming visual information was not being processed to the same extent as when full cognitive capacities were available. As event related negativity at frontal sites of the brain is associated with effortful cognitive processing, more positive fERPs at these locations may be indicative of reduced processing in areas linked to executive functions.

Most importantly, these metrics were sensitive to increases in cognitive load prior to changes in hazard perception performance. This work demonstrates that the analyses of fERPs may not only provide insight into perceptual but also the cognitive processes involved in visual processing.

\section{Pre-target activity of V1 linked to occurrence of express latency saccades}

\author{
Kayeon Kim, Choongkil Lee \\ Seoul National University, Republic of Korea \\ kayeonlo@snu.ac.kr
}

It has been shown that the variability in cortical activity before appearance of a saccade target is related to saccade latency and occurrence of express latency saccades in human [e.g., Bompas et al., 2015, Neuroimage, 107, 34-45]. In the current study, we examined the neural activity of primary visual cortex (V1) while two monkeys performed a gap saccade task, known to reduce saccade latency and increase occurrence of express saccades. Results indicated that the changes in neural latency and firing rate of spike activity evoked by the saccade target accompanied the gap effect and differentiation of express and regular latency saccades. During pre-target period, the level of both spike activity and spectral power of LFP were lower before express compared to regular latency saccades. These results extend the link between V1 activity and saccade latency [Lee et al., 2010, J Neurophysiol, 105, 255672 ], and support the idea that the pre-target activity of visual cortex can predict the latency of upcoming visually-guided saccades and occurrence of express saccades in identical stimulus conditions. 
Talks

\title{
The COGAIN Eye Data Quality Project
}

Room: HS 32

\author{
Revisiting Eyetracker Noise Estimators \\ Dixon C. Cleveland ${ }^{1}$, Fiona B. Mulvey ${ }^{2}$, Jeff B. Pelz ${ }^{3}$, COGAIN \\ Eye Data Quality Standardisation Committee ${ }^{4}$ \\ ${ }^{1}$ LC Tecnologies, Inc., United States of America \\ ${ }^{2}$ Humanities Laboratory, Lund University, Sweden \\ ${ }^{3}$ Carlson Center for Imaging Science, Rochester Institute of Technology, United States of America \\ ${ }^{4}$ COGAIN Association \\ dixon@eyegaze.com
}

Reliably detecting eye-gaze events (e.g., fixations, saccades, pursuit, blinks) in eyetracker recordings depends strongly on intrinsic instrument noise and precision. A key goal of COGAIN's Eye Data Quality Committee is to reach consensus on unbiased measures of eyetracker precision. We discuss three methods of characterizing eyetracker noise: the standard deviation (SD) of samples within a temporal window, the RMS of consecutive sample-tosample distances (RMS(S2S)), and the bivariate contour ellipse area (BCEA) typically used to measure 'fixational stability' in clinical research, and advocated as an eyetracker precision measure by Blignaut \& Beelders (2012). All approaches are in use, each with relative strengths and weaknesses. SD and BCEA assume the underlying signal is relatively stable within the sampling window, so it is important to select windows free of drift and (micro)saccades, but the eyetracker noise being estimated can itself make it difficult to identify such stable periods. The RMS(S2S) method significantly reduces the impact of erroneously including drift and small saccades within the measurement window, but this approach is particularly sensitive to sample rate and the spectral content of the noise, such that lowfrequency noise components and/or high sample rates can lead to significant underestimates of system noise. 


\title{
Modeling of settings for event detection algorithms based on noise level in eye tracking data
}

\author{
Raimondas Zemblys ${ }^{1,2}$, Kenneth Holmqvist ${ }^{1}$, Dong Wang ${ }^{3}$, Fiona Mulvey ${ }^{1}$, Jeff Pelz ${ }^{3}$, Sol \\ Simpson ${ }^{4}$ \\ ${ }^{1}$ Lund University, Sweden \\ 2 Siauliai University, Lithuania \\ ${ }^{3}$ Rochester Institute of Technology, US \\ 4 iSolver Software, Canada \\ r.zemblys@tf.su.It
}

Event detection depends critically on the precision of the eye tracking data. It is known that adjusting settings in commercial event detection algorithms can alleviate effects of noise, but not which settings are optimal. Taking real and very precise eye movement data from 36 participants, we have produced artificial trials with 40 different noise levels. These data sets were processed by the SMI event detection algorithms (both I-VT and I-DT), and by custom implementations of Tobii and SR Research algorithms. We compare the resulting saccades and fixations to a baseline of events from the best existing algorithm (Larsson et al., 2015) processing the original data.

We present regression models that show how the optimal algorithm settings depend on the precision in data. Preliminary linear mixed model analysis show that: For SMI I-VT, 17 $\mathrm{deg} / \mathrm{s}$ is an optimal threshold for noise-free data; A linear increase is needed with increasing noise; Significantly lower increase in threshold is needed for higher sampling rates for SMI I-VT; Merging of adjacent fixations (an optional stage in Tobii and SR-Research algorithms) leads to a more accurate estimation of average number of fixations, has minor effect on fixation durations, and does not affect saccade measures.

\section{Eye data quality and event detection: A comparison of algorithm performance across spectral noise characteristics}

\author{
Jeff B. Pelz ${ }^{1}$, Fiona B. Mulvey ${ }^{2}$, Dixon C. Cleveland ${ }^{3}$
}

${ }^{1}$ Carlson Center for Imaging Science, Rochester Institute of Technology, United States of America

${ }^{2}$ Humanities Laboratory \& Dept. of Psychology, Lund University, Sweden

${ }^{3}$ LC Technologies, Inc. Fairfax, United States of America pelz@cis.rit.edu

Eyetrackers vary widely, each with their own advantages and disadvantages in terms of what measures are possible from the system, and consequently, what kinds of applications they are most appropriate for. Most studies or applications of eye tracking rely on parsing the eye movement signal into events for analysis. In this study, we investigate the effect of data quality on the performance of event detection algorithms using a dataset collected for the COGAIN Eye Data Quality Standardization project. This dataset includes controlled recordings from a DPI eyetracker and 12 commercially available remote and tower-mounted video-based eyetrackers. We took the most stable and precise recording segments from the DPI eyetracker, and add spectral noise modeled on noise components measured from each observer per eyetracker. Each modeled dataset is submitted to commonly used event detection algorithms, including those implemented in an open source python library (Perrone, 2013). The events detected in the modeled datasets with added spectral noise are compared to those of the original DPI recordings. We show differences in the results from parsing algorithms with varying eye data quality, and report the most robust algorithms for various system and recording profiles. 
Talks

The effect of sample selection methods on data quality measures and on predictors for data quality

Kenneth Holmquist ${ }^{1}$, Raimondas Zemblys ${ }^{1}$, Dixon C. Cleveland ${ }^{2}$, Fiona B. Mulvey ${ }^{1}$, Josh Borah $^{3}$, Jeff B. Pelz ${ }^{4}$

${ }^{1}$ Lund University, Sweden

${ }^{2}$ LC Technology, United States of America

3 Applied Systems Laboratories, United States of America

${ }^{4}$ Rochester Institute of Technology, United States of America Kenneth.Holmqvist@humlab.lu.se

Sample selection is important for event detection, calibration of eye trackers and data quality assessment from eye movement data. As part of the Cogain EDQ work, we apply six different sample selection methods on raw data collected from up to 194 participants fixating 49 points on 12 remote and tower-mounted eye trackers (the DPI, SR EyeLink, SMI HiSpeed 240, 1250, RED250, RED500 and REDm, Tobii TX300, T60 XL, x2-60, the LC EyeFollower and the EyeTribe), and calculate precision, accuracy and track loss from the selected raw data samples. We can show that the average and median accuracy and precision vary with the sample selection method, reflecting how each method deals with correction saccades, noise and inaccuracies. We then apply linear mixed effects models to calculate significant data quality predictors (mascara, eye lash direction, pupil size, eye colour, position on the screen, etc.) from the same data, and can show that overall, the same predictors of data quality turn out significant irrespective of sample selection method. In around $10-20 \%$ of the cases, the data quality and robustness results for specific eye trackers vary across sample selection methods. These deviations reveal a rich interaction between sample selection and data quality. 


\section{How reliable is my eye-movement data? Results of system comparison and participant characteristics-based prediction of data quality from the COGAIN Eye Data Quality Project's Standardisation Committee.}

Fiona B. Mulvey ${ }^{1}$, Niels Landwehr ${ }^{2}$, Josh Borah ${ }^{5}$, Dixon C. Cleveland ${ }^{6}$, Markus Joos ${ }^{9}$, Kara Latorella $^{7}$, Jeff B. Pelz ${ }^{4}$, Sol Simpson ${ }^{3}$, Dong Wang ${ }^{4}$, COGAIN

Eye Data Quality Standardisation Committee ${ }^{8}$

${ }^{1}$ Humanities Laboratory \& Dept. of Psychology, Lund University, Sweden

${ }^{2}$ Department of Computer Science, University of Potsdam, Germany

3 iSolver Software, Canada

${ }^{4}$ Center for Imaging Science, Rochester Institute of Technology, United States of America

${ }^{5}$ Applied Science Laboratories, Washington State University, United States of America

${ }^{6}$ LC Technologies, Inc., Fairfax, United States of America

7 NASA Langley Research Center, United States of America

8 The COGAIN Association

${ }^{9}$ Interactive Minds, Dresden, Germany

Fiona.Mulvey@humlab.lu.se

Eye data quality varies significantly between eyetracking systems, and with the individual characteristics of the recorded eye, the recording environment, and operator. The quality of the signal has important repercussions for which eye movement measures can be validly reported, and hence, what conclusions can be validly drawn from them. While the data quality requirements vary with research question or application, reliable and unbiased reporting of data quality across systems and research results requires large-scale, controlled data collection and standardized measures. We report data quality in terms of spatial accuracy and spatial and temporal precision across commercial systems, across screen area, as well as system robustness to individual variation. We explore the use of machine learning techniques to infer predictive models of data quality as a function of employed systems, operator, and characteristics of the recorded eye across 12 tower mounted and remote eye tracking systems and up to 194 participants, with dedicated and universal methods. This work is output of the COGAIN Eye Data Quality Standardization Committee - a collaboration between manufacturers and researchers to agree unbiased measures and provide open-source tools to the eye tracking community toward transparency of eye data quality and prediction of sources of variance thereof. 


\title{
Wednesday, August $19^{\text {th }}, 8.50$ am -10.30 am \\ In memory of Keith Rayner
}

\author{
Room: HS 7
}

\section{Information Uptake during Reading is Effective but not always Accurate}

\author{
Albrecht W. Inhoff, Julie M. Gregg \\ Binghamton University, State University of New York, United States of America \\ inhoff@binghamton.edu
}

Keith Rayner's work showed that the extraction of linguistic information and oculomotor programming are tightly coordinated during reading. The current study examined the scope of this coordination, in particular, whether information uptake is also coordinated with reading accuracy. Participants read experimental and filler sentences while eye movements were recorded. Eye movements during the reading of experimental sentences were used to obtain six oculomotor measures, three indexing information uptake (skipping rate, saccade size, and gaze duration) and three potentially indexing information correction (refixation rate, regression rate, and re-reading time). Two non-oculomotor measures, obtained for different texts, indexed reading accuracy. One was the rate of correct responding to yesor-no question after filler sentence reading, another was the accuracy with which readers answered four-choice multiple-choice questions after the reading of a short story. Path analyses showed that oculomotor measures that are related to the uptake of information did not predict reading accuracy. Corrective oculomotor movements during experimental sentence reading, by contrast, did predict accuracy. The results suggest that the programming of eye movements to new words is designed so that information uptake will be fluent - but not necessarily accurate. Functionally distinct oculomotor responses are used to improve the representation of linguistic content. 


\title{
English Compound Word Recognition during Reading: An Eye Movement Investigation
}

\author{
Barbara Juhasz \\ Wesleyan University, Middletown, CT, United States of America \\ bjuhasz@wesleyan.edu
}

Recording eye movements is a very useful tool for exploring word recognition during reading (Rayner, 2009). Juhasz and Rayner (2003) demonstrated that a reader's past experience with a word affects the amount of time it takes to recognize that word, as indicated by effects of both rated word familiarity and age-of-acquisition (AoA) on fixation durations. The present study further explored the influence of past experience on word recognition by employing English bilexemic compound words. While previous studies have used eye movements to examine English compound word processing, most of these have focused on assessing lexical decomposition by manipulating lexeme frequencies (e.g. Andrews, Miller, \& Rayner, 2004, Juhasz, Starr, Inhoff, \& Placke, 2003). In comparison, the current study examined the impact of additional compound word attributes on eye movements during reading. A subset of 120 compound words from a recent rating study conducted by Juhasz, Lai, and Woodcock (in press) were selected and embedded in neutral sentences. Rated variables such as compound word familiarity and compound AoA influenced fixation durations on the compound words over and above compound word length, frequency, and lexeme frequencies. These results suggest that a reader's experience with compound words over their lifespan influences compound processing.

\section{Opening the Door on Cloze}

\section{Timothy J. Slattery}

Bournemouth University, UK tslattery@bournemouth.ac.uk

The predictability of a word in a sentence influences not only how long the eyes stay fixated on that word but also whether or not the eyes skip the word during first pass reading (Balota, Pollatsek, Rayner, 1985; Rayner, Slattery, Drieghe, Liversedge, 2011; Rayner\& Well, 1996; Sheridan \& Reingold, 2012). In fact, word predictability is a central variable in computational models of eye movements during reading (EZ-Reader, SWIFT, Glenmore). Traditionally, word predictability estimates are derived from the Cloze task which involves subjects iteratively guessing the next word in a sentence given all the prior words. However, estimates can also be obtained using Kneser-Ney smoothed N-gram models of text (Angele, Schotter, Slattery, Tenebaum, Rayner, 2015). Cloze task data are commonly dichotomized into correct and incorrect guesses of a word. The mean of this dichotomized variable is then taken as the words predictability. This technique discards much of the variability inherent in Cloze data. Here I suggest that we can use latent semantic analysis (Landauer, Foltz, Laham, 1998) to retain more of the information available in Cloze data and show how this additional information can be useful in explaining reading fixation durations. 
Talks

\title{
Computational Modeling of Reading: The "Whole Burrito" Erik D. Reichle \\ University of Southampton, United Kingdom reichle@soton.ac.uk
}

A large number of computational models have been developed to explain the various cognitive processes involved in reading (Reichle, 2015), including word identification (e.g., Perry et al., 2007), sentence processing (e.g., Lewis \& Vasishth, 2005), the representation of discourse (e.g., Frank et al., 2003), and how the systems that mediate these processes interact with the perceptual and motoric systems that guide the eyes during reading (e.g., Reichle et al., 2012). In this talk, I will describe efforts to develop a more comprehensive description of reading by embedding word-identification, sentence-processing, and discourserepresentation models within the framework of the E-Z Reader model of eye-movement control during reading (Reichle, 2015). The goal of this work has been to develop a computational framework for simulating on-line and off-line reading behaviors during reading (e.g., the patterns of eye movements made during reading, the content of the text that is later remembered), and by doing so provide a theoretical framework for better understanding both reading as a whole and the mutual constraints that word, sentence, and discourse processing impose on each other.

\section{Semantic Preview Benefit in Reading and Keith Rayner's More Recent Contribution to the Field of Eye Movements in Reading}

\author{
Elizabeth R. Schotter ${ }^{1}$, Simon P. Liversedge ${ }^{2}$ \\ 1 University of California, San Diego, United States of America \\ 2 University of Southampton, United Kingdom \\ eschotter@ucsd.edu
}

Semantic preview benefit in reading is controversial because it seems to depend on (at least) the language being read, visual properties of the text (e.g., initial letter capitalization), the relationship between preview and target, and semantic constraint due to prior sentential context. We will discuss three experiments that have been published using the boundary paradigm (Rayner, 1975) with four preview conditions (identical, synonym, semantically associated, and unrelated) crossed with a manipulation of sentence context (neutral vs. constrained). Across these studies a complex pattern of eye movement behavior emerges; we found a first-pass, apparent, preview benefit for semantically associated previews in constrained contexts that went away in later measures (e.g., total time). These data suggest sentence constraint generates expectations about what kinds of words, rather than which particular word, is likely to appear. These data are compatible with the E-Z Reader model that stipulates early oculomotor decisions reflect "hedged bets" that a word will be identifiable, while later reading behavior reflects comprehension failure associated with regressions and re-reading. 


\title{
Eye movements in new media and film
}

\author{
Room: HS 30
}

\section{List or grid? Eye movement assessment of the influence of the presentation format on decision making during Web searching}

\author{
you jia Zeng, Chen-Chao Tao \\ National Chiao Tung University, Taiwan, Republic of China \\ jayljill@hotmail.com
}

Web search can be viewed as a decision-making process. People explore different search results, evaluate and narrow possible ones, and finally choose the best matching search results. One of the key issues regarding Web search is whether the presentation format of search results influences decision making. Especially the list and grid formats - the most popular formats used in electronic commerce - treat the position of search results in different ways. For the list format the position implies the ranking, while for the grid format the position is simply a place. Forty participants took part in an eye tracking experiment, asking them to perform six search tasks with the list or grids formats. The results show that the position in the list format affects total fixation duration (the higher, the longer) and decision, whereas the position in the grid fails to affect total fixation duration and decision. In line with the decision making and eye movements literature, the chosen search results have longer first fixation duration and total fixation duration. Moreover, the decision was determined by interaction of total fixation duration and first fixation duration. It is concluded that the presentation format influences decision making during Web search.

\section{Reading for Comprehension versus Skim Reading on the Web: How Skim Reading is informed by Hyperlinks. \\ Gemma Fitzsimmons, Mark J. Weal, Denis Drieghe \\ University of Southampton, United Kingdom \\ G.Fitzsimmons@soton.ac.uk}

Studies of reading have focused on reading behaviour when participants read a single, mono-coloured sentence for comprehension. However, everyday reading behaviour such as reading hypertext on Webpages entails people reading or skim reading passages of text containing links in a colour, different from the main text. We recorded participants' eye movement behaviour and asked them to read for comprehension or skim read pages from Wikipedia that were modified to include target words. Target words were either hyperlinked or not, and were either a high- or a low-frequency word. When skim reading, participants read faster and skipped more words, even though comprehension was comparable across the tasks. Linked words were skipped less often than unlinked words when skimming, revealing that participants used the coloured words as 'anchor' points for scanning strategies. Frequency effects were observed during reading for comprehension but not during the skimming task, except when the words were hyperlinked, indicating more advanced lexical processing for these words. Results are discussed in terms of task effects on eye movements during reading and the necessity to also study reading behaviour in more realistic settings. 
Talks

\title{
Animated adverts impair children's online reading and text comprehension
}

\author{
Nils Holmberg, Kenneth Holmqvist, Helena Sandberg \\ Lund University, Sweden \\ nils.holmberg@kom.lu.se
}

In this study we examined the effects of animated adverts on children's online reading. We conducted an eye-tracking experiment with authentic web pages to assess whether (1) advert animation primarily affects overt visual attention, or (2) animated adverts primarily affect text comprehension, or (3) both eye movements and comprehension are affected. 59 children in third grade (9-year-olds) participated in the study. The stimulus material consisted of 6 factual texts that were presented on a news website. Online banner adverts were presented in a column on the right side of the texts. The adverts were presented in two conditions: static and animated. After reading each text the children provided answers to multiple choice comprehension questions. The results showed that advert animation affected several text processing measures, such as fixation duration and regressive saccades. This effect was stronger among children with poor gaze control (as measured with an antisaccade task). More importantly, however, the present study showed that animated adverts had a significant negative effect on children's text comprehension. The study discusses how these findings could be used to regulate commercial content on children's websites. Also, suggestions for using individual differences in eye movement measures to predict reading comprehension are explored.

\section{An eye-tracking comparison of cognitive load and flow status in reading e-books with printed words, spoken words, and portrait Gloria Yi-Ming Kao \\ National Taiwan University of Science and Technology, Republic of China gloriakao@gmail.com}

This study adopted eye-tracking technique and questionnaires to document and analyze if students with different cognitive styles would have different behaviors while reading three distinct types of e-books. We used "Little Red Riding Hood" as the learning content and designed three reading modes: graphic mode (picture + printed words), reading mode (picture + spoken words), role-playing mode (picture + printed words + portrait), to observe students' cognitive load and flow status. As a pilot study, we observed the behavior of six graduate students and found (1) most participants experienced flow in the reading mode, but not in the graphic mode; no specific flow status was identified in the role-playing mode; (2) All participants described the story as clear and understandable in the graphic and roleplaying mode, but found the storyline in the reading mode to be confusing; (3) Visualizers tended to have less cognitive load than verbalizers while reading all three types of e-book; (4) In the graphic mode, visualizers had more fixations in text than verbalizers; but no significant difference was found for fixations in the reading mode. In the role-playing mode, participants' fixations mostly focused on text and picture with only a few fixations on portraits. 


\section{Analog versus digital: How human perception adapts to new film aesthetics}

Miriam Laura Loertscher ${ }^{1,2}$, David Weibel ${ }^{1}$, Barbara Flueckiger ${ }^{3}$, Simon Spiegel ${ }^{3}$, Pierre Mennel $^{2}$, Fred W. Mast ${ }^{1}$, Rudolf Groner ${ }^{1}$, Christian Iseli ${ }^{2}$

${ }^{1}$ University of Bern, Switzerland

2 Zurich University of the Arts, Switzerland

${ }^{3}$ University of Zurich, Switzerland

miriam.loertscher@psy.unibe.ch

The digital revolution changed film production in many ways. But so far, nobody has compared complete narrative films captured with analog and digital cinematography empirically. In two cinema experiments (356 participants in experiment 1, 137 participants in experiment 2), we tested whether digital and analog recording methods are perceived differently. Eye tracking analysis of 67 participants was carried out to compare the perception of typical film scenes. The two capturing technologies produced similar emotional and immersive experiences during digital projection. The study revealed significant differences in the memory of visual details with higher recall scores for the digitally captured versions. Moreover, the mechanical projection of celluloid film produced higher levels of emotional reactions. New possibilities of analyzing film perception are discussed. 
Talks

\title{
Reading: Influences of context II
}

\section{Room: HS 31}

\section{Words in context: The effects of word length, frequency and predictability on brain responses during natural reading}

\author{
Sarah Schuster, Stefan Hawelka, Florian Hutzler, Martin Kronbichler, Fabio Richlan
}

Centre for Cognitive Neuroscience, Austria

Sarah.Schuster@stud.sbg.ac.at

Word length, frequency and predictability are the most influential linguistic variables in reading and have been well-documented in the context of eye movement (EM) studies. At the neural level, however, findings are mostly based upon single-word presentations and hence generalisability to natural reading is questionable. The present study investigated these effects on brain responses during natural reading by simultaneously recording bloodoxygen-level dependent (BOLD) signals and EM. In a multiple regression analysis, we observed that increasing word length was associated with increasing activation in early visual areas associated with local feature and global shape processing. Additionally, word length elicited a U-shaped modulation within the brainstem (i.e., the paramedian pontine reticular formation), with 6-to-8-letter words eliciting the least activity. This structure has been linked to the control of horizontally directed EM. For word frequency and predictability, we observed activation in a partly overlapping left-lateralized network related to lexico-semantic processing. This network encompassed occipito-temporal, middle and superior temporal and inferior frontal regions which exhibited an increase in activation with decreasing frequency and predictability. Our findings provide a crucial extension to the scarce evidence gained from single-word studies which advance our knowledge of the neural mechanisms that mediate the cognitive processes of reading. 


\title{
Animacy-based predictions show delayed effects in non-competitive environments
}

\author{
Franziska Kretzschmar ${ }^{1,2}$, Svenja Lüll ${ }^{1}$, Ina Bornkessel-Schlesewsky ${ }^{2,3}$, Matthias \\ Schlesewsky ${ }^{1,3}$ \\ 1 Johannes Gutenberg University Mainz, Germany \\ 2 Philipps University Marburg, Germany \\ ${ }^{3}$ University of South Australia, Australia \\ kretzsc@uni-mainz.de
}

In sentence comprehension, verb semantics and the animacy of verb arguments are major sources of predictions about the assignment of thematic roles. Previous studies found immediate effects of animacy on eye movements, but only tested the influence of animacy in transitive environments that promote competition for agenthood (Traxler et al. 2002, Mak et al. 2006). Here, we present a reading study investigating the strength of animacy as a predictive cue in simple intransitive clauses without argument competition. 84 participants read 36 German sentences derived from a 2x2x3 design, with verb type as between-subjects factor. We fully crossed animacy of the subject, verb preference for animate vs. inanimate subjects (A tourist/freighter swims/drifts across the lake), and word order. Subjects occurred preverbally or in one of two different postverbal word orders. This allowed us to test whether animacy cue strength varies contingent upon the preceding context. ANOVAs revealed spatio-temporally delayed effects. Animacy mismatches did not affect immediate processing of subject or verb in any word order, and instead prolonged reading times only for clause-final prepositional phrases. This effect was more pronounced for verbs preferring animate subjects. Thus, in non-competitive environments animacy has a reduced impact on eye movements in reading.

\section{Reading sentences of uniform word length Michael G. Cutter, Denis Drieghe, Simon P. Liversedge The University of Southampton, United Kingdom M.G.Cutter@soton.ac.uk}

We examined the effect of removing within sentence word length variability on saccadic targeting during reading. Participants read sentences comprised entirely of three-, four- or five-letter words, and sentences with a combination of these lengths. We hypothesised that saccadic targeting may adapt to these novel conditions, such that the saccadic range bias (McConkie et al., 1988) would be modulated by the length of words within the uniform sentences. We found that the saccadic range bias did adapt, such that the extent to which participants would under- or overshoot the centre of a word from a particular launch site was systematically modulated by the length of the words within a sentence. This adaptation occurred regardless of whether all thirty sentences from a single condition were presented in a block (Experiment 1) or interspersed randomly with each other and filler items (Experiment 2), suggesting that the saccadic targeting system can adapt to novel conditions near instantaneously. In addition, our manipulation had significant effects on word skipping, such that short words were skipped less frequently in uniform rather than non-uniform sentences. Finally, reading was slower in the uniform than non-uniform sentences, most likely due to the relative naturalness of the different sentence types. 
Talks

\author{
Using a dichoptic moving window presentation technique to \\ investigate binocular advantages during reading \\ Mirela Nikolova ${ }^{1}$, Stephanie Jainta ${ }^{2}$, Hazel I. Blythe ${ }^{1}$, Simon P. Liversedge ${ }^{1}$ \\ ${ }^{1}$ University of Southampton, United Kingdom \\ 2 2. LeibnizResearch Centre for Working Environment and Human Factors (IfADo) \\ M.Nikolova@soton.ac.uk
}

Reading comes with a clear binocular advantage, expressed in shorter fixation times and fewer regressions in binocular relative to monocular visual presentations. Little is known, however, about whether the cost associated with monocular viewing is more pronounced in the encoding of foveal information or in obtaining a preview benefit from upcoming parafoveal text. In the present sentence reading experiment, we developed a novel dichoptic binocular gaze-contingent moving window technique to selectively manipulate the amount of binocular information available in the fovea and parafovea on a fixation-by-fixation basis. This technique allowed us to pinpoint the exclusive influence of disrupting binocular fusion during direct fixation and during parafoveal pre-processing of upcoming text. Sentences were presented (1) binocularly; (2) monocularly; (3) with monocular text to the left of fixation (4) monocular text to the right of fixation; or (5) with all words other than the fixated word presented binocularly. A robust binocular advantage occurred for average fixation duration and regressions. While there was a limited cost associated with monocular foveal processing, the restriction of parafoveal processing to monocular information was particularly disruptive. The findings demonstrate the importance of a unified binocular input for pre-processing text to the right of fixation.

\title{
Orthographic neighborhood effects in L1 and L2 reading Cop Uschi, Nicolas Dirix, Wouter Duyck \\ Ghent University, Belgium uschi.cop@ugent.be
}

Both orthographic neighborhood density and frequency have been shown to facilitate or inhibit visual word recognition, dependent on task, instructions and/or stimuli (for a review see Andrews, 1997). Van Heuven, Dijkstra \& Grainger (1988) were the first to show a crosslingual neighborhood effect in Dutch-English bilinguals: the number of Dutch neighbors slowed down response times to English target words in a lexical decision task.

In a replication of their study we used the same stimuli but with correct neighborhood density and frequency values. We obtained different results from the original study, but these were still indicatory for a within- and cross-lingual neighborhood size effect.

A natural reading database of eye movements for Dutch-English bilinguals showed both facilitatory and inhibitory cross-lingual neighborhood (frequency) effects for L1 reading and L2 reading depending on word frequency, length and bigram frequency of the target word. These results show that the cross-lingual neighborhood effect is robust and not limited to the lexical decision task. 


\title{
Saccades and perception
}

Room: HS 32

Stimulus duration and compression of space

Sabine Born ${ }^{1,2}$, Hannah M. Krüger ${ }^{1}$, Patrick Cavanagh ${ }^{1}$

${ }^{1}$ Centre Attention \& Vision, Laboratoire Psychologie de la Perception, Université Paris Descartes, France

${ }^{2}$ Equipe Cognition Visuelle, Faculté de Psychologie et des Sciences de l'Education, Université de Genève, Switzerland

sabine.born@unige.ch

Stimuli flashed just before, during or after a saccade are mislocalized towards the saccade target, a phenomenon known as saccadic compression of space. The effect is typically attributed to saccade-specific extra-retinal signals interacting with visual input. Questioning this account, we have recently demonstrated compression of space in the absence of saccades: brief probes were attracted towards a visual reference when preceded or followed by a mask. Usually, compression effects are observed for flashed probes of 8-25 ms duration. Here, we examined the effect of longer probe durations on mislocalizations during saccades and masks. In Experiment 1, participants localized probes flashed at the end of a saccade or just after presentation of a full-screen mask. Probe duration varied between 17-100 ms. Short stimuli were strongly compressed, medium durations reduced the effect and long durations removed compression altogether. In Experiment 2, we analyzed the time course of compression and found that mislocalizations are strongest at saccade or mask onset. Even the long $100 \mathrm{~ms}$ probe now showed some compression in the mask, but not the saccade condition. Results are interpreted within a common framework for saccade and mask-induced effects, explaining compression in terms of correspondence matching, probe visibility and weak motion transients.

\section{On the Perception of Space Following Saccadic Adaption}

\author{
Hannah M. Krüger, Patrick Cavanagh, Thérèse Collins \\ Laboratoire Psychologie de la Perception,Université Paris Descartes 5, France \\ hannah.kruger@parisdescartes.fr
}

Changing the location of a saccade target mid-flight is a powerful way to change saccade metrics [McLaughlin, 1967]. Studies have shown that perceptual localisation is affected by saccadic adaptation if the probe is presented just before or after an adapted saccade. Here we show that saccadic adaptation also has an effect on perception of space in the adapted zone even in the absence of a saccade to that location. Participants were adapted to two peripheral locations in the upper and lower right quadrant such that saccadic amplitude to one right quadrant was shortened and lengthened to the other. Following saccadic adaptation participants judged the direction of apparent motion of a probe appearing between the upper and lower quadrant. Compared to preadaptation responses, a significant shift of perceived direction of motion was found in the same direction as the adaptation. The finding suggests that the perception of space is affected by the saccade system. 
Talks

\title{
Integration of pre-saccadic peripheral and post-saccadic foveal information follows relative reliabilities
}

\author{
Alexander C. Schütz, Christian Wolf \\ Justus-Liebig Universität Gießen, Germany \\ Alexander.C.Schuetz@psychol.uni-giessen.de
}

Humans identify interesting objects with peripheral vision and use eye movements to project them onto the fovea to receive high-acuity information. Although there is evidence that pre-saccadic peripheral and post-saccadic foveal information is integrated (Demeyer, De Graef, Wagemans \& Verfaillie, 2010), this has not been assessed with respect to formal models of signal integration (Ernst \& Banks, 2002).

Observers foveated peripherally appearing plaid stimuli and judged the orientation of the vertical component in a $2 \mathrm{AFC}$ task. To measure peripheral and foveal perception separately, plaids were visible either before or after the saccade for the same duration. To measure the integrated percept, plaids were visible throughout the trial but were exchanged during the saccade. We manipulated relative reliability of peripheral and foveal information by varying the foveal contrast in three steps. To manipulate relative biases, peripheral orientation could either be identical to foveal orientation or shifted to either side by $2.5^{\circ}$.

As predicted, the weighting of peripheral information increased with decreasing foveal reliability. Moreover, reliabilities of the combined percept were higher than for peripheral or foveal perception alone. These results suggest that pre-saccadic peripheral and postsaccadic foveal information are integrated into a combined percept according to their relative reliability.

\section{Attentional competition across the saccade depends on visual stability: Evidence from the blanking paradigm Christian H. Poth ${ }^{1,2}$, Arvid Herwig ${ }^{1,2}$, Werner X. Schneider ${ }^{1,2}$ \\ ${ }^{1}$ Center of Excellence Cognitive Interaction Technology (CITEC), Bielefeld University, Germany \\ ${ }^{2}$ Department of Psychology, Bielefeld University, Germany c.poth@uni-bielefeld.de}

Every saccade shifts the retinal image of its target object, but this mostly goes unnoticed. Instead, we perceive visual stability across the saccade. This perception conceals experimentally induced intrasaccadic object displacements. However, displacement identification is improved by a manipulation breaking transsaccadic visual object stability (Deubel, Schneider, \& Bridgeman, 1996), namely by introducing a brief blank screen between eye-landing and onset of the postsaccadic object. Here, we ask how such blanking affects postsaccadic object recognition. Observers made a saccade to a peripheral object which was displaced during the saccade. This object reappeared either immediately after the saccade or after a blank interval. Within the object, a letter was briefly presented (terminated by a mask). Observers reported displacement direction and letter identity in different blocks. Blanking improved displacement identification but impaired postsaccadic letter recognition. Breaking visual object stability is thought to make displacements accessible by preventing updating of pre- with postsaccadic representations. Therefore, separate representations of the same external object are preserved, enabling displacement perception. Our results demonstrate that breaking visual object stability across the saccade has beneficial effects on displacement perception while simultaneously impairing postsaccadic object recognition. This impairment might reflect attentional competition between separate transsaccadic object representations (Schneider, 2013). 


\title{
Visions of the Future: Action outcome prediction revealed by eye-movements
}

\author{
Christina Ursula Pfeuffer ${ }^{1,2}$, Lynn Huestegge ${ }^{1}$ \\ 1 University of Würzburg, Germany \\ 2 University of Freiburg, Germany \\ christina.pfeuffer@uni-wuerzburg.de
}

Humans are goal-directing their actions by predicting the consequences of their actions and executing those actions that produce the desired outcomes (e.g., Hommel et al., 2001). This implies the occurrence of expectations regarding future action consequences prior to acting (action effect anticipations). While there is ample behavioral evidence supporting this idea, there is yet no on-line measure of action effect anticipation. In the present study, we analyzed anticipatory eye movements towards predictable action outcomes in basic manual key press tasks. The results suggest that humans perform anticipatory saccades towards lateralized action effects prior to the occurrence of these action effects. More specifically, we demonstrate that anticipatory saccades not only occur between action and effect, but also prior to action execution during the phase of response preparation. Furthermore, we show that these anticipatory saccades do not only reflect the anticipation of the action effect stimuli, but are directly linked to action execution. These findings do not only provide further support for theories of action effect anticipation, but also demonstrate that eye-movements are a valuable on-line tool for deciphering the temporal dynamics of action effect anticipation. 


\title{
Thursday, August $20^{\text {th }}, 8.50$ am -10.30 am
}

\section{Triple P: Pre-, peri- and post-saccadic perception}

\section{Room: HS 7}

\author{
Transaccadic spatial stability and pre-saccadic perception \\ Paola Binda ${ }^{1}$, Guido Marco Cicchini ${ }^{2}$, David C. Burr ${ }^{2,3}$, M. Concetta Morrone ${ }^{1,4}$ \\ ${ }^{1}$ Universita' di Pisa, Italy \\ ${ }^{2}$ CNR, Istituto di Neuroscienze Pisa, Italy \\ 3 Universita' di Firenze, Italy \\ ${ }^{4}$ IRRCS Stella Maris Calambrone, Pisa, Italy \\ p.binda1@in.cnr.it
}

Eye movements pose major problems to the visual system, because each new saccade changes the mapping of external objects on the retina. While continuously visible objects are perceived as stable in spite of the displacement of their retinal image, perisaccadic flashes are mislocalized - usually compressed toward the saccade target. We investigated the relationship between these two phenomena. Our results show that a stimulus flashed just before saccade onset can be drawn toward another flashed stimulus presented pre-or postsaccadicaly. The interaction field is oriented along the retinotopic trajectory of saccadeinduced motion, which suggests a spatiotopic mechanism for trans-saccadic integration, consistent with the properties of "remapping cells". Both flashed stimuli and stable visual references exert a powerful influence over perisaccadic localization, which can be interpreted as the signature of trans-saccadic spatial binding for the same object. We jointly test the two phenomena in a condition where visual references can be tightly controlled: a dark anechoic room. In complete darkness with acoustic-driven saccades, both the compression toward the saccade target and the interaction between perisaccadic flashes decreases dramatically. This suggests a strong link between transient perisaccadic illusions and the stability of perception for the rich visual scene we are usually exposed to. 


\title{
A predictive copy of the motor command influences visual stability across saccades
}

\author{
Antimo Buonocore, David P. Melcher \\ Center for Mind/Brain Sciences, University of Trento, Rovereto, Italy \\ antimo.buonocore@unitn.it
}

Our perception of the surrounding environment remains stable despite the fact that we continuously change the retinal position of input by rapid gaze shifts (saccades). There is a long-standing debate whether or not visual stability depends on an active mechanism using an efference copy of the impending saccadic motor command or passive mechanisms, such as interference or masking by the post-saccadic stimulus. Many behavioural studies showing changes in perception around the time of saccades are consistent with a predictive mechanism, but previous attempts to test this hypothesis have been limited by the inability to selectively interfere with the efference copy of the eye movement command in humans. We applied for the first time a non-invasive technique to suppress saccadic eye movements by behavioral means. By using a saccadic inhibition (SI) paradigm, which delays saccadic onset, we were able to dissociate programming from the actual movement for three paradigms that have been suggested to involve efference copy: mislocalisation, saccadic suppression and double-step saccades. Our results suggest that the role of prediction of our own movements influences both perceptual experience and action. These findings have important implications for the larger problem of how sensory and motor systems are integrated in neural circuits.

\section{How transsaccadic predictions shape the perception of shape}

\author{
Arvid Herwig, Katharina Weiß, Werner X. Schneider \\ Bielefeld University, Germany \\ aherwig@uni-bielefeld.de
}

Human vision is characterized by consistent shifts between fixations and saccadic eye movements. With each saccade, internal object representations change their retinal position and spatial resolution. This raises the question as to how extra-foveal perception is affected by upcoming saccadic eye movements. Recently, we suggested that saccades are accompanied by a prediction of their perceptual consequences-i.e., the foveation of the target object (Herwig \& Schneider, 2014, JEP:G). Accordingly, extra-foveal perception should be biased toward previously associated foveal input. In the present study, we tested whether the extrafoveal perception of visual shape is partly based on predicted postsaccadic foveal input. Participants first underwent an acquisition phase, where one out of two objects changed its shape during saccades. In the following test phase, participants had to judge the shape of briefly presented peripheral saccade targets. Targets were perceived as less curved for objects which previously changed from more circular in the periphery to more triangular in the fovea compared to objects which did not change during acquisition. Likewise, shapes were perceived as more curved for objects which previously changed from triangular to circular. This result indicates that the extra-foveal perception of shape is specifically biased toward previously associated postsaccadic foveal input. 
Talks

\title{
Cortical feedback to primary visual cortex during interhemifield saccades
}

\author{
Grace Edwards ${ }^{1,3}$, Petra Vetter ${ }^{2}$, Lars Muckli ${ }^{3}$ \\ 1 CERCO, University of Toulouse - Paul Sabatier, France \\ 2 Medical School \& Swiss Center for Affective Sciences, University of Geneva, Switzerland \\ 3 Institute of Neuroscience \& Psychology, University of Glasgow, United Kingdom \\ grace.edwards@cerco.ups-tlse.fr
}

The primary visual cortex (V1) is an integration region for feedforward visual input and cortical feedback signals. Some cortical feedback to V1 is thought to be predictive of sensory input. A critical assumption is that feedback descending the cortical hierarchy can update to new retinotopic locations during eye-movements. We aim to provide evidence for feedback relocating to new retinotopic coordinates across saccades in V1. Feedback relocation was found using the apparent motion illusion in psychophysical and fMRI experiments. Apparent motion is the illusion of a moving token between two rhythmically flashing stimuli. Perception of illusory motion signifies the engagement of a cortical feedback signals as a moving token is internally constructed from stationary input. We find the spatiotemporally specific feedback signals are remapped to new coordinates in V1 within 50-100 ms of saccade offset. Feedback relocation was also found by investigating a non-stimulated target region in V1 while a complex scene was presented in the contralateral hemisphere using fMRI. By incorporating interhemifield saccades we found that contextual information of the image was fed-back to aid image processing after saccade at the new retinotopic position. In summary, these experiments demonstrate cortical feedback in V1 during saccades, which effects post-saccadic perception.

\section{Saccadic influences on vision beyond early stages of sensory encoding Sven Ohl ${ }^{1,2}$, Martin Rolfs ${ }^{1,2}$ \\ ${ }^{1}$ Humboldt Universität zu Berlin, Germany \\ ${ }^{2}$ Bernstein Center for Computational Neuroscience Berlin, Germany sven.ohl@bccn-berlin.de}

Saccades bring parts of a visual scene into foveal sight enabling their encoding at highest spatial resolution. Here we asked whether saccades also affect the processing of visual stimuli that have already disappeared from view. In a series of experiments, we briefly presented arrays of oriented stimuli and-several hundred milliseconds later-prompted saccades to a random location in the array. A subsequent probe instructed participants to report the orientation of one randomly selected stimulus in the array-probing memory either at the same location (congruent) or at a different location than the saccade target (incongruent). By contrasting congruent with incongruent trials, we reveal a strong influence of saccades on processing of past visual stimuli, with superior performance in congruent trials. This influence was strongest right after stimulus offset, still present after $800 \mathrm{~ms}$, and vanished for longer delays (Experiment 1). Moreover, it occurred for small set-sizes (Experiment 2 and 3) and stimuli presented below perceptual threshold (Experiment 3). We propose that saccades impose a natural bottleneck for the selection and transfer of volatile representations in iconic and fragile visual short-term memory into more durable representations. We discuss the role of actions at the interface of retro-perception and visual memory. 


\title{
Reading: General II
}

\author{
Room: HS 30
}

\section{The development of eye-movements in three different orthographies: an eye-tracking investigation}

\author{
Sascha Schroeder ${ }^{1}$, Tuomo Häikiö ${ }^{2}$, Ascensión Pagán ${ }^{3}$, Jonathan H. Dickins ${ }^{3}$, Jukka \\ Hyönä $^{2}$, Simon P. Liversedge ${ }^{3}$ \\ 1 Max Planck Institute for Human Development, Germany \\ 2 University of Turku, Finland \\ 3 University of Southampton, United Kingdom \\ sascha.schroeder@mpib-berlin.mpg.de
}

Languages vary in their orthographic transparency, i.e., how easy it is to derive the pronunciation of a word from its spelling: English is opaque, Finnish transparent, and German has an intermediate position. Orthographic transparency has been argued to be a main determinant for cross-linguistic differences in reading development. Here, we present results from a study investigating the development of eye-movements in Finnish, German, and English. Identical stimulus materials were used that comprised 96 sentences that were carefully translated and back-translated into all three languages. Word length (short vs. long) and frequency (high vs. low) were manipulated orthogonally for 48 target words.

Thirty 4th grade children and 25 adults were assessed in each language. Results show that eye-movement characteristics varied substantially with orthographic transparency: In gaze duration and total reading time, length effects were generally stronger in Finnish and German than in English; and were moderated by word frequency in Finish and German. These differences were more pronounced for children than adults. This indicates that reading becomes automatized earlier in transparent orthographies, but that sublexical decoding remains a dominant processing strategy even in adults. Generally, our results show that children's eye-movements continuously adapt to the constraints of their linguistic environment.

\section{Chinese Individuals Use Orthography Knowledge in Spoken Word Recognition}

\author{
Qingqing $\mathbf{Q u}^{1}$, Wei Shen ${ }^{1}$, Mengsi Wang ${ }^{2}$, Xingshan $\mathbf{L i}^{1}$ \\ ${ }^{1}$ Key Laboratory of Behavioral Science, Institute of Psychology, Chinese Academy of Sciences, Beijing, \\ People's Republic of China \\ 2 Department of Psychology, Wuhan University, People's Republic of China \\ quqq@psych.ac.cn
}

Do speakers of all languages activate orthography when they recognize spoken words? A large number of studies provide evidence for the influence of orthography in spoken word recognition, but the bulk of evidence comes from alphabetic languages in which the relation between orthography and phonology is strong and relatively transparent. By contrast, in languages with non-alphabetic scripts such as Chinese, the orthography-phonology connection is largely opaque, raising the possibility that speakers of these languages do not use orthographic information in spoken word recognition. We used the printed-word variant of the visual-world paradigm combined with eye tracking technique to investigate whether spelling affects Chinese spoken word recognition. Chinese native speakers listened neutral sentences including target words while viewing arrays of four printed words which included a target word, a phonological competitor, an orthographic competitor, and an unrelated distractor. Our study showed that listeners fixated on both phonological competitors and orthographic competitors more than on unrelated words. Our findings thus constitute clear evidence that orthographic information constrains spoken word recognition, even in Chinese. 
Talks

\title{
Orthographic Similarity and Relative Frequency Influence Competition during Visual Word Identification
}

\author{
Julie M. Gregg ${ }^{1}$, Ralph Radach ${ }^{2}$, Albrecht W. Inhoff ${ }^{1}$ \\ ${ }^{1}$ Binghamton University, State University of New York, United States of America \\ 2 University of Wuppertal, Germany \\ jgregg3@binghamton.edu
}

Words with higher frequency neighbors are difficult to identify (e.g., Perea \& Pollatsek, 1998). Using a modified version of the visual world paradigm (VWP) with visually presented words, the present experiment investigated the competition process underlying this effect. Participants viewed a briefly presented and subsequently masked target word, which was one member of a pair of orthographic neighbors differing in frequency. The target was then selected from among four alternatives (shown in different screen quadrants): the target, its orthographic neighbor, an orthographic onset competitor, and an unrelated control. Targets eventually emerged as the strongly preferred object of viewing, but there was competition from orthographically similar alternatives early in the selection phase, particularly when the target had been the lower-frequency member of a target pair. Selection latencies were also longer for lower-frequency targets, suggesting that selection of a lower-frequency target from its competitors was relatively difficult. Participants also made more selection errors for lower- than for higher-frequency targets, and erroneous selection an orthographic neighbor was the most common error. Our time-locked analyses of eye movements in the VWP suggest that orthographic similarity and relative frequency influence early stages of lexical competition, and that this can sometimes result in identification errors.

\section{The Influence of Word Length, Spatial Extent, and Initial Bigram Characteristics on Eye Movement Control During Reading: Evidence from Arabic}

\author{
Ehab W. Hermena, Denis Drieghe, Simon P. Liversedge \\ University of Southampton, United Kingdom \\ ewh1g09@soton.ac.uk
}

Through the use of a proportional font, which is more natural-looking in Arabic compared to monospaced fonts, we manipulated the number of letters (5 versus 7 ) and the spatial extent (wide versus narrow) of a word embedded in a frame sentence. The results replicate previous findings in other alphabetic languages that the number of letters influences fixation durations, whereas saccade targeting (skipping and saccade amplitude) is more influenced by the word's spatial extent. Distributional analyses indicated that initial fixations landed, on average, 0.6 of a character further into words starting with the article prefix (Arabic "the"). We conducted a second experiment comparing initial fixation locations in 6- and 7-letter words beginning with either the highest frequency prefix (the article the); a high-frequency prefix (Arabic "for the"); or no prefix. Initial fixations landed further into words in both prefixed conditions when measured in characters. However, due to the high frequency prefixes consisting of comparatively narrow letters, saccade lengths and fixation locations were practically identical in both experiments compared to non-prefixed conditions when initial landing position was calculated in terms of spatial extent (pixels). We conclude that saccade targeting in Arabic is minimally influenced by prefix frequency. 


\section{Reading in Agglutinating Languages: The Role of Morphology on Eye Movements \\ Cengiz Acartürk, Özge Nilay Yalcin \\ Cognitive Science, Middle East Technical University, Turkey acarturk@metu.edu.tr}

The agglutinative morphology of Turkic languages (e.g., Turkish, Uyghur) provides an appropriate basis to study the role of complex morphological structures on eye-movement patterns in reading. We examined the influence of morphological complexity on a set of eye-movement parameters while controlling linguistic parameters, such as word length, word frequency and initial letter-bigram frequencies of a set of target words. Three sevenletter, target-word conditions (root, single-suffixed, double-suffixed) were designed while controlling the frequency of the target words, the initial letter-bigrams of the target words, as well as the sentence frame prior to and one-word posterior to the target words.

The experiment with 45 Turkish native speakers revealed significant relationships among LS (Launch Site) on the pre-target word n-1, FLP (Fixation Landing Position) on the target word n, Launch Site (LS) on n, and FLP on the post-target word n+1. As for the first fixation durations (FFD) on the target word, both launch site $(\chi 2(1)=12.22, \mathrm{p}<0.001)$ and morphological structure $(\chi 2(1)=5.32, \mathrm{p}=0.02)$ had a significant influence on FFD. In particular, morphologically complex target words resulted in multiple fixations on the first-pass reading of $\mathrm{n}$, which also led to shorter FFD on $\mathrm{n}$. 


\title{
Talks
}

\section{Eye movements and attention}

\author{
Room: HS 31
}

\author{
Tracking down attentional templates \\ Artem Belopolsky, Chris Olivers \\ VU University Amsterdam, Netherlands \\ artem.belopolsky@gmail.com
}

\begin{abstract}
Attentional templates are target templates that help guide visual search. Recent studies using electrophysiology suggested that observers initially keep the template in working memory but may quickly offload it to long-term memory if target repeats. In the present study the process of learning the target template was investigated using eye-tracking. Participants had to search for a Landolt $C$ with a gap in eight possible orientations. The target of search switched every seven trials. Before the start of each trial participants received a cue, whose color and shape indicated the upcoming target, paired with a distractor cue. The cues were small enough that the gap could not be discerned from fixation. The results showed that the first saccade was directed to the cue on $85 \%$ of trials and this did not change as a function of target repetition. Fixation durations decreased with target repetition, suggesting reduced reliance on working memory. When symbolic cues were used, both orienting to the cue and fixation durations on the cue decreased as a function of target repetition. The results suggest that learning of attentional template depends on how efficiently the world can be used as the "external memory".
\end{abstract}

\section{Information gain does not modulate the facilitation of saccades by a perceptual task

\author{
Christian Wolf, Alexander C. Schütz \\ Justus-Liebig University Giessen, Germany \\ Christian.Wolf@psychol.uni-giessen.de
}

Saccades project interesting objects onto the fovea to acquire high-acuity information. Their latencies are reduced if participants perform a perceptual task at the saccade target (Montagnini \& Chelazzi, 2005). So far, it is unknown if this facilitation is a general task effect, or whether it depends on the information gain by the eye movements.

Here, we tested if latencies are sensitive to pre-saccadic peripheral visibility, post-saccadic foveal visibility or the ratio of both, i.e. the information gain. Observers foveated peripherally appearing plaid stimuli and judged the orientation of the vertical component. We varied the contrast ratio of both components while the overall contrast remained constant. To independently manipulate peripheral and foveal visibility, we exchanged plaids during saccades.

In a control condition without perceptual task, latencies were not influenced by the plaids contrast ratio. Consistently with previous reports, latencies were reduced by about $40 \mathrm{~ms}$ with perceptual task, even when fast saccades were maladaptive because peripheral exceeded foveal visibility. There was a tendency towards reduced latencies with poor peripheral visibility. Results indicate that perceptual tasks generally facilitate eye movements, independent of visibility. This suggests that eye movement latencies are not equally modulated by informational as by motivational gain (Milstein \& Dorris, 2007). 


\section{Near-perfect decoding of visual attention with pupillometry Sebastiaan Mathôt ${ }^{1}$, Jean-Baptiste Melmi ${ }^{1}$, Lotje van der Linden ${ }^{1}$, Stefan Van der Stigchel ${ }^{2}$ \\ ${ }^{1}$ Aix-Marseille University, CNRS, LPC UMR 7290, Marseille, France \\ 2 Dept. of Experimental Psychology, Helmholtz Institute, Utrecht University, Netherlands s.mathot@cogsci.nl}

When the eyes are exposed to light, the pupils constrict. This is the well known pupillary light response. What is less well known is that the light response is not a simple reflex, but is modulated by visual attention: When you covertly attend to a bright stimulus, your pupil constricts relative to when you attend to a dark stimulus. Here we describe a humancomputer interface that is based on this principle, i.e. decoding the focus of covert visual attention with pupillometry. Participants fixate in the center of a display, and select (i.e. covertly attend to) one of several stimuli presented in a circular arrangement. Each stimulus is presented on a background with alternating brightness. Small changes in pupil size reflect the brightness alternations of the selected stimulus' background, and this allows us to determine which stimulus is selected on a trial-by-trial basis. Mean selection accuracy is around $90 \%$ for untrained participants (based on a preregistered training program with 10 participants), and is nearly perfect ( $>99 \%$ ) for trained participants. An extension of this technique even allows unconstrained text input through a virtual keyboard. We discuss potential applications, such as communication with locked-in patients and ultra-secure password input.

\section{Covert selection and suppression as determinants of oculomotor behaviour: An eye-tracking and EEG study \\ Matthew D. Weaver, Clayton Hickey, Wieske van Zoest \\ University of Trento, Italy \\ matthew.weaver@unitn.it}

In a concurrent eye-tracking/EEG study we explored how mechanisms of attention in visual cortex contribute to oculomotor control. Participants made eye movements to a linesegment target embedded in a search display also containing a salient distractor. Target and distractor salience was manipulated by varying degree of orientation offset from a homogenous background. Results show that successful saccades to less-salient targets were preceded by a.) a target-elicited contralateral negativity (N2pc), reflecting the deployment of covert attention to the target, and b.) a distractor-elicited contralateral positivity (Pd), reflecting distractor inhibition. When the eyes were mis-deployed to the distractor, these components were absent or reduced. Counter-intuitively, overt and covert selection of the target was predicted by increased amplitude of high alpha-band activity $(12-17 \mathrm{~Hz})$ in the prestimulus interval, suggesting a role in establishing control. A second experiment demonstrates that saccade timing relative to $\mathrm{Pd}$ is crucial in determining whether the trajectory of target-directed saccades deviates toward or away from distractors, indicating the level of suppression applied to irrelevant salience. Our results demonstrate the contribution of presaccadic visuo-cortical mechanisms in the control of oculomotor selection and establish the utility of concurrent eye tracking/EEG recording in directly linking selection performance with neural mechanisms. 
Talks

\title{
Visual search for targets both in and out of predictable locations within routes
}

\author{
Oliver Tew ${ }^{1}$, Hayward J. Godwin'1, Matthew Garner², Julie A. Hadwin ${ }^{3}$, Simon P. \\ Liversedge $^{1}$, Nick Donnelly ${ }^{1}$ \\ ${ }^{1}$ Centre for Vision and Cognition, University of Southampton, United Kingdom \\ ${ }^{2}$ Psychology and Medicine, University of Southampton, United Kingdom \\ ${ }^{3}$ Developmental Brain-Behaviour Laboratory (DBBL), Psychology, University of Southampton, United \\ Kingdom \\ ot1e13@soton.ac.uk
}

Real-world visual searches for targets are aided by familiarity with scene contexts. Here we explore how knowing the scene within a larger environment aids target detection. We report two experiments where sets of 40 static scenes were taken along a route and presented either in an order consistent with moving along the route or in a randomised order. The task was to identify if scenes had targets (tools) present in them over eight repetitions. In Experiment 1, targets were equally likely to be present in any scene across repetitions of the route. Our results demonstrated a learning effect in accuracy and eye movement data across repetitions but no effect of presentation order (consistently vs. randomly arranged scenes). In Experiment 2, we employed the same basic design but targets were fixed to particular scenes, so if a target was present in one scene, there would always be a target somewhere in repeated presentations of that scene. The data from Experiment 2 are currently being analysed. We anticipate that comparisons across experiments will reveal an influence of route familiarity such that target detection improves as the ability to predict the presence of forthcoming targets increases. 


\title{
Mobile eye-tracking: Tools and findings
}

\author{
Room: HS 32
}

\author{
Rummage Searching for Targets by Individuals, Teams and Experts \\ Charlotte A. Riggs ${ }^{1}$, Carl M. Mann ${ }^{1}$, Hayward J. Godwin ${ }^{1}$, Sarah Smith ${ }^{2}$, Michael \\ Boardman ${ }^{2}$, Simon P. Liversedge ${ }^{1}$, Nick Donnelly ${ }^{1}$ \\ 1 University of Southampton, United Kingdom \\ 2 Defence Science and Technology Laboratory \\ c.a.riggs@soton.ac.uk
}

Rummage search is the visual and physical search in real world environments for targets such as weapons or drugs. Rummage search was explored in novice individuals (Experiment 1 ) and novice two-person teams (Experiment 2). Participants sought targets relating to an armed robbery scenario placed in four rooms of a residential house. Eye movements were recorded using head mounted eye trackers. Some targets were plainly visible whereas others were hidden, or could only be found through physical exploration. We also compared novice performance against the performance of highly trained rummage search experts. We draw three conclusions. First, novice searchers, whether searching as individuals or pairs, did not rummage search for targets exhaustively. Second, the failure to search exhaustively was associated with failures to fixate possible target locations and to physically search possible target locations completely. Third, the failures were not a function of the task load of rummaging in complex environments but of task expertise and skill, as working as a pair did not lead to differences in overall accuracy, search time, or exhaustiveness of search. We conclude that effective rummage searching is a skill to be trained.

\section{Do it yourself! Active object handling leads to faster search times. Dejan Draschkow, Melissa Le-Hoa Võ \\ Goethe University Frankfurt, Germany draschkow@psych.uni-frankfurt.de}

Interacting with the environment and observing others interact with it is an essential part of everyday life. To investigate how physically engaging with objects influences memory and therefore subsequent search for these objects, we constructed a real-world paradigm in which participants equipped with a mobile eye tracker either actively placed objects in shelves (Active condition), or passively observed the experimenter place objects (Passive condition). In an initial Construction phase, participants were asked to conduct the two tasks in random order for a total of 120 objects distributed in 4 shelves. In the subsequent Search phase, participants had to search trial-by-trial for all objects in the same shelf before moving to the next one. During construction of the subsequent search display, actively manipulated objects received shorter fixations and dwell times than passively observed objects. Nevertheless, time to first fixation on targets in the Search session was significantly reduced for objects that were previously actively placed by the participant. Interestingly, while initially longer search times decreased for "passive" objects, searches for previously actively manipulated objects remained stable. We argue that active object placement leads to a stronger object-location binding, which subsequently guides search beyond episodic memory generated during repeated real-world searches. 
Talks

\title{
Automatic Analysis of a Mobile Dual Eye-Tracking Study on Joint Attention \\ Patrick Renner ${ }^{1}$, Thies Pfeiffer ${ }^{2}$, Nadine Pfeiffer-Leßmann ${ }^{1}$ \\ ${ }^{1}$ SFB 673, Faculty of Technology, Bielefeld University, Germany \\ 2 CITEC, Faculty of Technology, Bielefeld University, Germany \\ prenner@techfak.uni-bielefeld.de
}

Our research aims at cognitive modelling of joint attention for artificial agents, such as virtual agents or robots. With the current study, we are focusing on the observation of interaction patterns of joint attention and their time course. For this, we recorded and analyzed twenty sessions of two interacting participants using two mobile binocular eye-tracking systems.

A key contribution of our work addresses methodological aspects of mobile eye-tracking studies with scene camera recordings in general. The standard procedure for the analysis of such gaze videos requires a manual annotation. This time consuming process often exceeds multiple times the duration of the original recordings (e.g. 30 times). This doubles if, as in our case, the gaze of two interlocutors is recorded simultaneously.

In our approach, we build upon our EyeSee3D approach for marker-based tracking and registration of the environment and a $3 \mathrm{D}$ reconstruction of the relevant stimuli. We extend upon the previous approach in supporting more than one participant and dynamically changing stimuli, here the faces and eyes of the interlocutors. The full analysis of the time course of both interlocutor's gaze is done in real-time and available for analysis right after the experiment without the requirement for manual annotation.

\section{Application of Markov chains in the analysis of data acquisition process in ship simulator environment}

\author{
Bartosz Muczynski, Maciej Gucma \\ Maritime University of Szczecin, Poland \\ b. muczynski@am.szczecin.pl
}

Human factor is said to be the main reason behind over $90 \%$ of all ship's collisions. Some studies stress the critical role of fatigue and sleep deprivation, as well as inadequate lookout and lack of experience and knowledge. At the same time, very few studies perceive officers as a part of a human-computer interaction process and take into account the increased cognitive load resulting from bad interface design and information overload. Previous research of the authors presented a method of using gaze data to evaluate officers' performance in marine simulator environment. Herein, we present how eye-tracking data can be used to describe the acquisition of the navigational data in relation to difficulty and complexity of the task, and how to model this process using Markov chains. To that end, a data acquisition process needs be considered as a stochastic memoryless process in which fixation location describes its state. This opens up a discussion about direct measurement of officer's working memory. Two cases are described: acquiring data from all available sources on the ship's bridge, and from a single interface only. 


\title{
Thursday, August $20^{\text {th }}, 1.20 \mathrm{pm}-3.00 \mathrm{pm}$ \\ Development of parafoveal processing during reading acquisition
}

\author{
Room: HS 7
}

\section{Does parafoveal processing require competence in basic word-decoding processes? A longitudinal developmental study of the perceptual span}

\author{
Jochen Laubrock, Johannes M. Meixner, Anja Sperlich \\ Universität Potsdam, Germany \\ laubrock@uni-potsdam.de
}

Does parafoveal processing require competence in basic word-decoding processes? How does the perceptual span change during the initial years of reading instruction? Is the perceptual span a stable indicator of reading competence, and how is it related to other tasks such RAN or psychometric reading measures? Are individual early reading deficits compensated or inflated over time? We report data from the first two installments of a longitudinal study of the perceptual span in German beginning readers. These were collected in the context of a large study measuring a wide array of psychological and developmental indicators, which will prospectively allow us to relate reading development to psychological well-being and educational trajectories. Cross-sectional results from T1 indicate that reading rate increased from Grades 1-3, with smaller increases for higher grades. Perceptualspan results showed the expected main effects of grade and window size. Critically, several measures indicate that the perceptual span only develops after basic reading processes are mastered. T2 Results nicely replicate the basic pattern of cross-sectional effects. Finally, eye tracking measures correlated substantially with psychometric reading test scores. Analyses of the intra-individual stability and developmental trajectories in these measures will be reported.

\section{Parafoveal pre-processing of word initial trigrams during reading in children}

\author{
Ascensión Pagán, Hazel I. Blythe, Simon P. Liversedge \\ University of Southampton, United Kingdom \\ a.p.pagan-camacho@soton.ac.uk
}

Although previous research has shown that letter position information for the first letter of a parafoveal word is coded less flexibly than internal letters in adults (Johnson, Perea \& Rayner, 2007; White et al., 2008), it is not clear how positional encoding operates over the initial trigram in both adults and children. This experiment explored the pre-processing of letter identity and position information of a parafoveal word's initial trigram by adults and children using the boundary paradigm during normal sentence reading. Seven previews were generated: Identity (captain); transposed letter and substituted letter nonwords in position 1\&2 (acptain-imptain); $1 \& 3$ (pactain-gartain) and $2 \& 3$ (cpatain-cgotain). Results showed a transposed letter effect (TLE) in position 13 for gaze duration in the pre-target word; and TLE in position 12 and 23 in single fixation and gaze durations in the target word for both adults and children. These findings suggest that children, similar to adults, extract initial letter identity and position information from the parafovea in a flexible manner using a spatial coding mechanism; supporting isolated word recognition models such as SOLAR (Davis, 1999) and SERIOL (Whitney, 2001). The present study extends previous evidence about letter position encoding in both adults and children. 


\title{
Development of children's parafoveal processing of phonological and orthographic information
}

\author{
Simon Patrick Tiffin-Richards, Sascha Schroeder \\ Max Planck Institute for Human Development, Germany \\ tiffin-richards@mpib-berlin.mpg.de
}

We present two measurement points of a longitudinal study of children's parafoveal processing of phonological and orthographic information. The sample of $\mathrm{N}=75$ German speaking children read sentences with embedded target nouns while their eye movements were recorded. Using the boundary eye tracking paradigm, we investigated phonological and orthographic preview benefit effects in the parafovea by experimentally manipulating pseudohomophone (PsH) and transposed-letter (TL) previews. The $60 \mathrm{TL}$ and $70 \mathrm{PsH}$ sentences were read by all children in the second half of their second year of schooling ( $\mathrm{M}$ age $=7.8$ years) and again in the second half of their third year ( $\mathrm{M}$ age $=8.8$ years). We found no significant TL or PsH preview benefit effects for children in second grade, suggesting that they do not yet have the necessary free cognitive resources for parafoveal processing, due to the high cognitive load of foveal processes. Preliminary data suggests that by third grade these children show a development of reading fluency, reading comprehension, and parafoveal phonological processing. Our results are consistent with the developmental view that beginning readers use available cognitive resources for parafoveal processing during reading as their reading skill improves and the cognitive load of foveal processing decreases.

\section{Parafoveal preprocessing of phonology during silent reading in typically developing readers and readers with Permanent Childhood Hearing Impairment

\author{
Hazel I. Blythe, Jonathan H. Dickins, Colin R. Kennedy, Simon P. Liversedge \\ University of Southampton, United Kingdom \\ hib@soton.ac.uk
}

Individuals with Permanent Childhood Hearing Impairment (PCHI) have phonological processing deficits, as well as marked literacy deficits. Eye movement research has shown that skilled adult readers pre-process phonological information from word N+1 during silent sentence reading (Pollatsek, Lesch, Morris \& Rayner, 1992). The extraction of phonological information from $\mathrm{N}+1$ is, however, constrained by the reader's skill and recent work suggests interesting differences between hearing and hearing-impaired readers (Chace, Rayner \& Well, 2005; Bélanger, Mayberry \& Rayner, 2013). Such processing has not, to date, been examined in developing readers. As part of a large-scale developmental investigation, we compared typically developing readers and developing readers with PCHI. In Experiment 1, eye movements were recorded as they read sentences containing three types of target words: correct words (e.g., church); pseudohomophones (e.g., cherch); and orthographic controls (e.g., charch). The visual similarity of the pseudohomophone-correct target pairs was also manipulated. In Experiment 2, these same manipulations were made in parafoveal preview using the boundary paradigm. The data showed differential patterns of sensitivity to our manipulations between the participant groups, and will be discussed in the context of theoretical standpoints on phonological processing in reading. 


\section{Recent developments in the research of children's parafoveal processing in reading}

Tuomo Häikiö

University of Turku, Finland tuilha@utu.fi

Since the seminal study of Rayner (1986), the parafoveal processing in children's reading was left virtually untouched for two decades despite the enormous potential of the methodology. In the recent years, interest in the topic has been resurrected, and starting with the Häikiö et al. (2009) paper there has been a surge of new studies examining the subject in greater detail, examining for instance the size of the perceptual span and transposed-letter effects of developing readers in both small-scale and large-scale experiments as well as in several languages, such as English and German. In the present talk, I will go over the short history of the studies in the area and will focus on the recent developments. It will be discussed how the use of techniques examining the parafoveal processing has advanced the knowledge of child reading. Topics covered include whether the parafoveal processing of children is mainly orthographic or phonological; whether early readers utilize parafoveal information in the same way as adults or whether they process parafoveal information in a qualitatively different way; and if the latter is true, when there is a shift towards more adult-like processing. 
Talks

\title{
Eye movements and attention in moving images
}

Room: HS 30

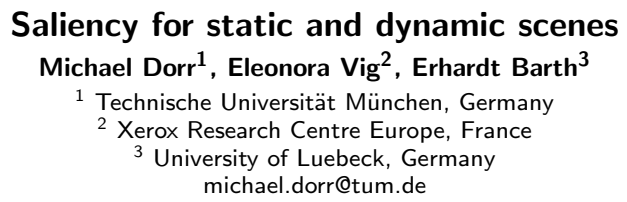

Image saliency computation, or the classification of image regions into interesting and uninteresting patches, is a highly active research field in Computer Vision. As a ground truth, images are typically labelled by human eye movements; this is based on the assumption that the human visual system has evolved, and thus been optimized, for perception and action in complex natural environments. However, the real world is not static and image motion is highly informative of other biological agents. Nevertheless, there has been much less research on video saliency because of the increased computational complexity.

Here, we review our work on modelling attention and eye movements in dynamic natural scenes, and how such models can improve performance in scene-understanding tasks such as action recognition. We will also report how established and well-performing models for image saliency can be used for dynamic content with little increase in computational cost by applying them to two-dimensional slices of the spatio-temporal volumes, for example in the $\mathrm{x}$-t domain.

\section{The “tyranny of film": Movie viewers' gaze minimally reflects differences in their comprehension processes}

\author{
Lester C. Loschky ${ }^{1}$, John P. Hutson ${ }^{1}$, Adam M. Larson ${ }^{2}$, Joseph P. Magliano ${ }^{3}$, Tim J. \\ Smith $^{4}$ \\ 1 Kansas State University, United States of America \\ 2 University of Findlay, United States of America \\ 3 Northern Illinois University, United States of America \\ ${ }^{4}$ Birkbeck, University of London, United Kingdom \\ loschky@ksu.edu
}

What is the relationship between eye movements and film comprehension? While reading research shows that eye movements and comprehension are strongly related, research on eye movements in film shows strong attentional synchrony, which might eliminate comprehension-based differences. We investigated this question in two experimental case studies (8 experiments), using clips from two movies, "Moonraker," and "Touch of Evil." Both studies manipulated context using what we call the "jumped-in-the-middle" paradigm: the Context condition saw more preceding film context than the No-context condition. Both studies found robust differences in viewer comprehension due to context, as measured by viewers' predictions of what would happen next in the film, and their event segmentation, but minimal corresponding differences in their eye movements using numerous measures. This is strong evidence for what we call "the tyranny of film," in that film-makers can control viewers' gaze regardless of variations in their higher-level understanding. This weak eye movements/comprehension relationship in film viewing suggests that normal film viewing is relatively passive, allowing gaze to be exogenously controlled. However, such exogenous control is heightened by film-making techniques, since attentional synchrony is greater in commercial films than in "natural movies" involving static camera shots of real-world scenes (Dorr et al., 2010). 


\title{
Hear my lips: Implicit detection of asynchronous audio-visual speech by eye movements
}

\author{
Tim J. Smith ${ }^{1}$, Jonathan P. Batten ${ }^{1}$, Katri Seppälä ${ }^{1}$, Rachael Bedford ${ }^{2}$ \\ ${ }^{1}$ Birkbeck, University of London, United Kingdom \\ 2 Institute of Psychiatry, Psychology and Neuroscience, King's College London, United Kingdom \\ tj.smith@bbk.ac.uk
}

When watching TV we often have the impression that poorly synchronised audio can "popout", drawing attention to the speaking actor's mouth. But how does such capture relate to the levels at which audiovisual asynchrony detection becomes explicit?

Participant eye movements were recorded while they watched an edited thirteen minute TV interview. Unbeknownst to participants, the audiovisual synchrony was manipulated across cuts to create eleven synch conditions between $-200 \mathrm{~ms}$ (audio first) and $+200 \mathrm{~ms}$ (video first). Each synch condition was presented twice with a return to $0 \mathrm{~ms}$ in between. Conditions gradually increased across the video, +40 to $+200 \mathrm{~ms}$, then decreased from $-40 \mathrm{~ms}$ to $-200 \mathrm{~ms}$. Following the initial free-viewing, participants were then instructed to explicitly identify whether the audio was in synch for a 5 s clip taken from the same interview under the same eleven synch conditions.

Analysis of participant eye movements during free-viewing revealed a significant bias towards the mouth and away from the eyes at $-120 \mathrm{~ms},+80 \mathrm{~ms}$ and $+120 \mathrm{~ms}$ asynchrony, levels at which asynchrony was not explicitly detected. These results indicate that asynchronous audiovisual speech captures overt attention even when the degree of asynchrony is subthreshold for explicit awareness and may be used as a measure of implicit asynchrony detection.

\section{Meaning, memory and individual differences in fixation during the viewing of dynamic scenes \\ Tom Foulsham \\ University of Essex, United Kingdom \\ foulsham@essex.ac.uk}

When viewing moving images, participants often look in the same place at the same time. In a range of studies, we have been examining how the meaning of key moments, as well as differences between observers, might affect this attentional synchrony. We have found systematic differences in social attention between younger and older observers watching dynamic crowd scenes, with older participants less affected by the characteristics of the people in the scene. While watching movie clips, convergence in fixation position is associated with explicit self-reports of "important" moments, such that attention seems to converge at meaningful times. The observers' sex and preferences may also contribute to inter-observer consistency, with participants who are more similar to each other being more likely to look in the same place at the same time. Additional analysis of eye movements during repeated viewings can give insights into the role of memory, as well as providing a test case for methods to quantify fixation similarity. Together, these examples show that measuring fixations in dynamic scenes can reveal much about active visual cognition. 
Talks

\section{The role of implicit visual memory for gaze guidance after cuts in edited moving images

\author{
Christian Valuch, Raphael Seywerth, Ulrich Ansorge \\ Faculty of Psychology, University of Vienna, Austria \\ christian.valuch@univie.ac.at
}

Visual search experiments suggest that eye movements in a given trial are biased for visual features and locations repeated from a previous trial. Such implicit memory could also contribute to gaze guidance after scene cuts in edited movies. We tested this hypothesis using a large set of street videos. From each video we generated two alternative shots which overlapped by precisely $50 \%$. Our experiments consisted of 160 two-shot sequences in which the first shot ended with a central fixation cross, and was followed by a second shot which was either: (a) a continuation of the first shot, (b) the alternative shot from the same source video (establishing a different viewpoint with partial overlap between shots), or (c) an unrelated shot. For each two-shot sequence, we analyzed how the first shot influenced observers' gaze in the second shot. Results showed a strong gaze preference for previously seen and repeated content which could not be explained by salient visual features alone. Especially in viewpoint shifts, observers' gaze clustered in overlapping regions of the shots within the first second after the cut. We conclude that gaze guidance by implicit memory enables instantaneous recognition of scene continuations and establishes perceptual relations across cuts. 


\title{
Eye movements, attention, and memory
}

\author{
Room: HS 31
}

\section{Updating of visual-spatial working memory across saccades}

\author{
Paul Boon, Artem Belopolsky, Jan Theeuwes \\ VU University, Netherlands \\ p.j.boon@vu.nl
}

\begin{abstract}
Visual-spatial working memory (VSWM) helps us to maintain and manipulate visual information in the absence of sensory input. Recent studies have proposed that it may be an emergent property of the oculomotor system. In the present study we investigated the relative role of retinal and extra-retinal signals in updating of working memory representations across saccades. Participants had to maintain a location in memory while making a saccade to a different location. During the saccade the target could be displaced, which was not perceived by the participants. After the saccade participants had to indicate the memorized location either by a match-to-sample task, a mouse click or by another saccade. The results showed a small but systematic bias of target displacement on localization in all response modalities, suggesting that postsaccadic retinal information has only a small effect on memory updating. The bias disappeared completely when no corrective saccades were made in the saccade localization condition. Taken together results suggest that VSWM strongly relies on the oculomotor system in updating working memory representations across saccades.
\end{abstract}

\section{Network analysis of anatomical connectivity reveals an information processing stream for memory-guided visual behavior Kelly Shen ${ }^{1}$, Gleb Bezgin ${ }^{1}$, Anthony R. Mclntosh ${ }^{1,2}$, Jennifer D. Ryan ${ }^{1,2}$ \\ 1 Rotman Research Institute, Baycrest, Toronto, Canada \\ 2 University of Toronto, Canada kshen@research.baycrest.org}

Visual behavior is guided by memories from previous experience and knowledge of the visual scene. A medial temporal lobe network mediates these memory processes. How information from this memory network influences oculomotor areas is not well understood. We examined the neuroanatomical basis for the routing of memory information to oculomotor structures. We derived a connectivity matrix using a database of macaque axonal tract tracing studies. The matrix included 73 cortical and subcortical ROIs from the visual, oculomotor and memory systems. We split the matrix graph into two processing streams using an iterative force-directed layout procedure. A visuo-oculomotor stream included visual and extrastriate cortices, area LIP, and SC. A memory stream included the hippocampus, and entorhinal and parahippocampal cortices. Among the oculomotor structures, FEF was assigned with the lowest probability to the oculomotor stream. Interestingly, dlPFC, SEF and ACC were assigned more often to the memory stream than the oculomotor stream. Moreover, FEF, dIPFC and SEF were well connected across streams. These regions may therefore serve as an anatomical interface between the oculomotor and memory systems. Our data are in line with previous neurophysiological studies that suggest a role for dIPFC, SEF, ACC and FEF in the cognitive control of gaze. 
Talks

\title{
Attentional Guidance by Simultaneously Active Working Memory Representations: Evidence from Competition in Saccade Target Selection
}

\author{
Valerie Margaret Beck, Andrew Hollingworth \\ University of lowa, United States of America \\ valerie-beck@uiowa.edu
}

The content of working memory (WM) guides attention, but there is debate over whether this interaction is limited to a single WM representation or functional for multiple WM representations. To evaluate whether multiple WM representations guide attention simultaneously, we used a gaze-contingent search paradigm to directly manipulate selection history and examine the competition between multiple cue-matching saccade target objects. Participants first saw a cue composed of two colors (e.g., red and blue) followed by two pairs of colored objects presented sequentially. For each pair, participants selectively fixated an object that matched one of the cue colors. Critically, for the second pair, the cue color from the first pair was presented either with a new distractor color or with the second cued color. In the latter case, if two colors in memory interact with selection simultaneously, we expected substantial competition from the second cued color, even though the first cued color was used to guide attention in the previous pair. Indeed, saccades for the second pair were more frequently directed to the second cued color object than to a distractor color object. This competition between cue-matching objects provides compelling evidence that both WM representations were interacting with and influencing attentional guidance.

\section{Priorities for representation: Task settings and object interaction both influence object memory}

\author{
Clare Kirtley, Benjamin W. Tatler \\ University of Dundee, United Kingdom \\ c.kirtley@dundee.ac.uk
}

Following an active task, the memory representations for used and unused objects are different. However, it is not clear whether these differences arise due to prioritising objects that are task-relevant, objects that are physically interacted with, or a combination of the two factors. The present study allowed us to tease apart the relative importance of task-relevance and physical manipulation on object representations. A paradigm was designed in which objects were either used in the course of a task (task-relevant); moved out of the way (taskirrelevant, but interacted with), or simply present in the environment (background). Participants' eye movements were recorded with a portable tracker during the task, and they received a memory test on the objects after the task was completed. Results showed that manipulating an object is sufficient to change how information is extracted and retained from fixations, compared to background objects. Task-relevance provides an additional influence: information is accumulated and retained differently for task-relevant manipulated objects than task-irrelevant manipulated objects. These findings demonstrate that representational priorities for objects are set both by whether we physically interact with an object, and the relevance of that object to our behavioural goals. 


\title{
False memory-guided eye movements: insights from the DRM-Saccade paradigm
}

\author{
Damien Litchfield ${ }^{1}$, Lauren Knott ${ }^{2}$, Tim Donovan ${ }^{3}$, Trevor Crawford ${ }^{4}$ \\ ${ }^{1}$ Edge Hill University, United Kingdom \\ ${ }^{2}$ City University London, United Kingdom \\ 3 University of Cumbria, United Kingdom \\ ${ }^{4}$ Lancaster University, United Kingdom \\ damien.litchfield@edgehill.ac.uk
}

The DRM-Saccade (DRM-S) task is a newly developed paradigm that bridges two wellestablished research methodologies: the pro/anti-saccade task and the Deese-Roedigger and MacDermot (DRM) paradigm, which reliably elicits false memories for critical nonpresented words at test. We investigated whether cognitive processes that produce true and false memories differ when recognition responses are eye movements (saccadic latency, errors, corrective saccades). 20 participants studied 24 DRM lists and we used a modified rulebased version of a pro/anti-saccade task to assess recognition of test words (seen, unseen, critical lure). Participant's memory of previously studied DRM words was the deciding rule as to whether they should look towards/away from a peripheral target to the left/right of test word. We manipulated Rule - whether participants should look towards/away from the target if they recognised the word or not, and Backwards Associative Strength of lists (High BAS, Low BAS), as High BAS increases false memories. Word type, Rule, and BAS modulated latencies and errors, but a key finding was that latency also dissociated false recognition of critical lures from true memory recognition, provided BAS was Low. We discuss how this DRM-S task provides new insights into memory-guided eye movements and false memory. 


\title{
Talks
}

\section{Eye movements: Faces}

\section{Room: HS 32}

\author{
The Facespan - the perceptual span for face recognition \\ Michael Papinutto ${ }^{1}$, Junpeng Lao ${ }^{1}$, Roberto Caldara ${ }^{1}$, Sébastien Miellet ${ }^{2}$ \\ ${ }^{1}$ University of Fribourg, Switzerland \\ 2 Bournemouth University, United Kingdom \\ michael.papinutto@unifr.ch
}

The perceptual span in reading - the region of effective vision during eye fixation - has been appraised almost 40 years ago by the pioneering work of Keith Rayner.

Surprisingly, the perceptual span for faces (the Facespan) has not been isolated yet, despite the on-going debate on information use for face recognition.

To address this question, we parametrically modulated 11 gaze-contingent moving-window apertures (Spotlights) during an old-new task on 220 observers. Spotlight performance was always statistically different from the efficient natural viewing condition. However, our results showed a parametrical decrease of the individual effect size of the Spotlight accuracy and reaction time; the larger the aperture the better the performance. A sigmoidal function isolated a plateau at a $17^{\circ}$ Spotlight aperture in this decrease, and was confirmed by eye movements analysis. We then applied a retinal filter to assess the quantity of intact information preserved at this aperture. This resulted in a Facespan of $3.5^{\circ}$ of visual angle. The Facespan size encompasses a face feature (e.g., an eye) plus immediate surroundings.

Our data show that in contrast with the discrete perceptual span in reading, information sampling for faces is better described by a gradient from fovea to periphery.

\section{Face recognition: The contribution of different input locations and input durations to recognition processes}

\author{
Charlotte Schwedes, Dirk Wentura \\ Saarland University, Germany \\ c.schwedes@mx.uni-saarland.de
}

In face recognition tasks, two fixations suffice to reach maximal performance. By disentangling the confound between the number of input locations and the input duration (Hsiao \& Cottrell,2008), the increase in performance could not solely be explained by longer input durations. The input from the second location plays a significant role. We were interested in the underlying processes and had the hypothesis that under equivalent input durations, input from a second location will boost recollection, resulting in better performance. Therefore, participants $(\mathrm{N}=49)$ familiarized themselves with a set of faces in a learning phase. In the following recognition test, faces were presented for: (1) one fixation location and the duration of one fixation; (2) one fixation location but the duration of two fixations; (3) two fixation locations and the duration of two fixations; (4) three fixation locations and the duration of three fixations. A remember/know/guess procedure followed. Maximum recognition performance was achieved with two fixation locations. Remember responses increased with longer input durations as well as more fixation locations. Know responses increased only with an additional input duration but not with an additional fixation location. These results are compatible to our hypothesis that a second input location boosts recollection. 


\title{
Idiosyncratic Visual Information Sampling Strategies regulate the Face Inversion Effect
}

\author{
Xinyi Ouyang ${ }^{1}$, Sébastien Miellet ${ }^{2}$, Junpeng Lao ${ }^{1}$, Roberto Caldara ${ }^{1}$ \\ 1 university of Fribourg, Switzerland \\ 2 Bournemouth University, United Kingdom \\ xinyi.ouyang@unifr.ch
}

The visual system actively and flexibly adapts information sampling to effectively achieve face recognition. However, when faces are inverted recognition performance dramatically decreases: the so-called face inversion effect (FIE). Yet, whether qualitative and/or quantitative changes in information sampling are at the root of the FIE is still debated. To clarify this issue, we implemented the gaze-contingent Expanding Spotlight technique (Miellet et al., 2013), while observers performed a delayed face-matching task with upright and inverted faces. A $2^{\circ}$ Gaussian aperture was centered on each fixation and expanded dynamically by 1 degree every $12 \mathrm{~ms}$. Importantly, we then used a Bayesian model to categorize the observers according to their information sampling strategy (i.e., global/central versus local/featural fixations). Recognition performance decreased for inverted faces in global observers only. Interestingly, only global observers qualitatively changed their fixation pattern, moving from the center to the mouth during inversion. Local observers instead constantly sampled their preferred facial features, using less information during inversion. Our data offer novel insights on the nature of the FIE, by showing how idiosyncratic visual information sampling strategies modulate the FIE. They also question the universality of this well-established phenomenon and reinforce the view of different routes for faces recognition.

\section{Cultural Diversity in Eye Movements is shaped by Nurture not Nature Roberto Caldara, Anne-Raphaëlle Richoz, Yingdi Liu, Junpeng Lao \\ Department of Psychology, University of Fribourg, Switzerland roberto.caldara@unifr.ch}

Humans adapt to their environment through a unique amalgamation of culture and biology. Historically, it has long been presumed that across cultures, all humans perceive and sample the world in a comparable manner. Recently, however, this notion has been disputed by evidence showing fundamental perceptual differences between people from Eastern (China, Korea and Japan) and Western cultures, for low- (reflexive saccades) and high-level (faces) visual information sampling strategies. Easterners perform global/central fixations towards the nose during face recognition and numerous express reflexive saccades. Westerners instead spread (local) fixations across the eye and mouth regions and execute fewer express reflexive saccades. Yet, whether these well-established cultural differences are related to nature or nurture remains unaddressed. We recorded the eye movements of culturally Westerners South Korean adoptees while they performed an old/new face recognition task and visually guided saccades. We then used a Bayesian model to quantify the local/global visual information sampling strategy for each observer. The South Korean adoptees perfectly fitted with the Western oculomotor behavior for face recognition, showing also few express reflexive saccades compared to Easterners. Altogether, our data show that nurture and not nature is responsible for cultural variations in eye movements. Culture finely tunes the visual system. 
Talks

\title{
Exploring Measures of Guidance in Visual Search for Multiple Unfamiliar Faces
}

\author{
Natalie Mestry ${ }^{1}$, Tamaryn Menneer ${ }^{1}$, Hayward J. Godwin ${ }^{1}$, Kyle R. Cave ${ }^{2}$, Nick Donnelly ${ }^{1}$ \\ ${ }^{1}$ University of Southampton, United Kingdom \\ 2 University of Massachusetts Amherst, United States of America \\ natalie.mestry@soton.ac.uk
}

In a previous study (Mestry et al., 2015, VSS) we showed a dual-target cost when searching for one of two unfamiliar faces compared to searching for either face alone. Target-distractor similarity was varied, with distractors formed by morphing between targets. By measuring eye movements we showed that search was serial and self-terminating and there was no guidance to targets in either single- or dual-target search. It is possible that the high level of task difficulty, due to high similarity between morphs, led to the dual-target cost and to the absence of guidance in the search for unfamiliar face targets. In the present study we repeat the experiment using un-morphed faces as stimuli but with visual similarity of targets to distractors determined by multidimensional scaling from fifty participants. Preliminary results suggest that while performance is considerably more accurate in this experiment than in the previous one, all other effects remain qualitatively similar. There appears to be no evidence of guidance in the proportion of fixations to targets or distractors. We conclude that the absence of guidance to unfamiliar faces, and the dual-target cost for faces, are fundamental limitations on face search. The applied implications of these limitations are significant. 


\title{
Thursday, August $20^{\text {th }}, 5.00 \mathrm{pm}-6.40 / 7.00 \mathrm{pm}$
}

\section{Reading: Parafoveal processing}

\author{
Room: HS 7
}

\section{Individual differences in parafoveal processing as a function of reading rate and visual acuity measures}

\author{
Sarah Risse \\ University of Potsdam, Germany \\ sarah.risse@uni-potsdam.de
}

Visual acuity rapidly decreases beyond the fovea. Consequently, the time to read words after they have been previewed in parafoveal vision should depend on how good their constituent letters were visible before fixation.

Eye movements of 60 participants were recorded while reading sentences that contained a gaze-contingent parafoveal preview manipulation in the boundary paradigm (Rayner, 1975). In addition, visual acuity indicators from the readers' visual span profiles (VSP) were collected in an independent experiment (cf. Legge, Mansfield, \& Chung, 2001).

Standard correlation analysis provided evidence for VSP parameters and preview benefit relating to individual reading rates, showing that subjects with higher scores in central vision read faster but simultaneously revealed less preview benefit. Moreover, there was no evidence for a direct link between preview benefit and parafoveal visual acuity in the right visual span. This rather unexpected finding was confirmed with linear mixed-effects models at the level of single fixation durations on the target word after the eyes crossed the boundary.

The present results suggest a more complex relationship between visual acuity, preview benefit, and reading rate than typically assumed. Parafoveal processing seems to have an independent effect on oculomotor control beyond an acuity-related slowing in parafoveal word recognition.

\section{Parafoveal Processing in Silent and Oral Reading of Chinese Sentences}

\author{
Jinger Pan, Jochen Laubrock, Ming Yan \\ University of Potsdam, Germany \\ jinger.pan@uni-potsdam.de
}

Using the gaze-contingent boundary paradigm, we investigated how reading mode (i.e., silent and oral reading) influences parafoveal semantic and phonological processing during the reading of Chinese sentences in two experiments. In silent reading, we observed semantic preview benefits in early and late processing in both experiments, whereas phonological preview effects only occurred in relatively late processing. In oral reading however, phonological preview information is obtained early on. Strikingly, we observed a semantic preview cost for single characters (Experiment 1 ) and a cancellation of semantic preview benefit for two-character words (Experiment 2) during oral reading, which we propose to be a result of increased preview duration in oral reading. Our results indicate that parafoveal semantic information can be obtained irrespective of reading mode, whereas parafoveal phonological information is processed more efficiently in oral reading. These results are in agreement with the notion that the Chinese orthography is well-optimized for fast access to semantic information. 
Talks

\title{
Acquisition of information from left of the current fixation in reading Ralph Radach ${ }^{1}$, Ronan Reilly ${ }^{2}$, Christian Vorstius ${ }^{1}$, Albrecht W. Inhoff ${ }^{3}$ \\ 1 University of Wuppertal, Germany \\ 2 National University of Ireland, Maynooth, Ireland \\ 3 State University of New York, Binghamton, United States of America radach@uni-wuppertal.de
}

The present work addresses the question to what extent the spatially distributed acquisition of linguistic information in reading extends to locations left of the current fixation (Binder, Pollatsek \& Rayner, 1999; Inhoff \& Radach, 2000). Participants were asked to read sentences containing two critical words, a low to medium frequency target adjective and a high frequency post-target noun. This combination should facilitate the continued acquisition of information from the left-of-fixation target word while the post target is fixated. Saccade contingent display manipulations were implemented to replace the target with a string consisting of either visually or dissimilar letters while the post target word was being fixated.

Results indicated that information was indeed acquired from the left parafovea. Readers more frequently executed a regressive saccade towards the target when it was masked with visually dissimilar letters. The primary effect of the less conspicuous similar letter masks was to increase word viewing times on the post target. This pattern of results is in harmony with parallel processing models of eye movement control in reading such as Glenmore and SWIFT. Future research will show to what extend the orthographic parafoveal post-view effect demonstrated here can be generalized to other types of linguistic information.

\section{On the peril of parafoveal masks and asset of the novel incremental boundary paradigm}

\author{
Florian Hutzler, Stefan Hawelka, Benjamin Gagl, Christina Marx, Sarah Schuster \\ University of Salzburg, Austria \\ florian.hutzler@sbg.ac.at
}

Parafoveal preprocessing is usually studied by the application of parafoveal maks. Its magnitude is estimated by comparing the masked preview with condition(s) in which parafoveal preprocessing of a (partially) valid preview is permitted. This experimental rationale relies on the implicit assumption that masks prevent parafoveal preprocessing, but that they neither facilitate nor interfere with the foveal processing of the target word, i.e., masks are considered to be neutral. This talk presents recent evidence from eye tracking and fixation-related potential studies disproving the implicit assumption about the neutrality of parafoveal masks. The serious limitations for the interpretability of experiments applying parafoveal masks are discussed. We introduce a novel incremental boundary technique as an alternative approach for investigating parafoveal preprocessing. This approach systematically varies the informational value (i.e, the salience) of parafoveal previews, thereby rendering the use of parafoveal masks unnecessary. A validation of this approach and example applications for the investigation of emerging parafoveal preprocessing in beginning readers and for the study of preview benefits in adult readers are presented. 


\title{
Parafoveal processing of contextually predictable words: Effects of reading proficiency
}

\author{
Aaron Veldre, Sally Andrews \\ University of Sydney, Australia \\ aaron.veldre@sydney.edu.au
}

This study investigated whether reading proficiency modulates the extraction of parafoveal information from contextually predictable words. Sentences were constructed in which constraining contextual information came either before or after a critical target word, such that the target was highly predictable or not predictable when it was encountered in first-pass reading. The parafoveal preview of the target was also manipulated to be identical, orthographically related, or unrelated. In order to capture individual differences in lexical precision, participants were assessed on measures of reading comprehension and spelling ability. In the average data, preview benefit was larger for predictable than unpredictable words. However, analyses including the individual difference measures revealed that this interaction was principally restricted to low proficiency readers. Higher proficiency readers showed equivalent preview benefits for predictable and unpredictable words. There was also evidence that the precision of skilled readers' lexical representations affected the probability of skipping predictable words. These results suggest that readers with imprecise lexical knowledge are more likely to rely on contextual information to supplement word identification during reading.

\section{Parafoveal Processing of Arabic Diacritical Marks}

Denis Drieghe, Ehab W. Hermena, Simon P. Liversedge

University of Southampton, United Kingdom

d.drieghe@soton.ac.uk

Diacritics are glyphlike marks on letters which convey vowel information in Arabic, thus allowing for accurate pronunciation and disambiguation of homographs. Arabic homographs are predominantly heterophones (sound different) with one version more common (predominant) than the other (subordinate). We presented native readers with such heterophonic homographs embedded in frame sentences, either with the predominant or the subordinate diacritisation pattern. Using the boundary paradigm, we presented previews of these words that contained either the correct diacritisation pattern; an inaccurate diacritisation pattern such that if the target had the predominant diacritisation pattern, the preview contained the subordinate pattern, and vice-versa; or no diacritics. The results showed that fixation durations were inflated on words with the subordinate diacritisation pattern, and that inaccurate preview of diacritics came with a cost but only when the subordinate target word was preceded by the dominant preview. Finally, the no-diacritics condition received slightly shorter fixation durations possibly due to effects of attentional capture from the parafoveally presented diacritics in the other conditions. All effects were more pronounced when the previous fixation was at a close launch-site. This experiment is the first to demonstrate effects of dominant versus subordinate diacritisation patterns and parafoveal processing of diacritics. 


\title{
Talks
}

\section{Saccades II}

Room: HS 30

\author{
Control of fixation duration by foveal and peripheral evidence \\ Casimir J.H. Ludwig ${ }^{1}$, George Stothart ${ }^{1}$, Craig Hedge ${ }^{2}$, Andy Skinner ${ }^{1}$ \\ ${ }^{1}$ University of Bristol, United Kingdom \\ 2 University of Cardiff, United Kingdom \\ c.ludwig@bristol.ac.uk
}

Saccade target selection is often modelled as a race to threshold between alternative motor programmes without consideration of foveal processing. In a series of experiments, observers performed a comparative tilt judgement. One pattern was presented at central fixation and two patterns appeared in the periphery. Observers compared the tilt of the central pattern with that of a peripheral target defined by luminance contrast. To identify the tilt of the peripheral target, observers had to select it on the basis of contrast and fixate the pattern. This paradigm enables independent manipulation of the foveal load (tilt offset from vertical) and peripheral evidence (contrast difference between target and nontarget). In some experiments we inserted temporal noise in both dimensions to identify the time windows of foveal analysis and target selection within a single fixation. We found that (i) fixation duration is predominantly influenced by foveal load; (ii) long fixations are associated with an attenuated uptake of early information; (iii) this attenuation was not due to the early evidence itself being undiagnostic, suggesting a role for variability in the observer's "pre-trial state". The minor influence of peripheral evidence on fixation duration is inconsistent with a race model of target selection and latency.

\section{Individual differences in the time-course of saccadic selection Wieske van Zoest \\ Center for Mind/ Brain Sciences, University of Trento, Italy Wieske.vanZoest@unitn.it}

Oculomotor selection has an idiosyncratic time-course: early selection is driven primarily by raw saliency, whereas late selection is guided by search templates. My colleagues and I have started to test the idea that this time-course can change as a function of experience and early developmental plasticity. We investigated whether selection dynamics are altered 1) in observers that have a background of increased visual training as a result of extensive action video-game (AVG) playing, 2) in deaf observers who rely heavily on information from the visual domain because of the absence of auditory input, and 3) across sexes. Our results show that relative to control groups, the time-course of oculomotor performance is speeded in AVG players, but slowed in deaf observers. Individuals that respond relatively slower in both groups are less influenced by stimulus-saliency than individuals that respond relatively fast. However, the time-course of visual processing fundamentally remains consistent across groups: the function shifts in time, but does not change qualitatively. In contrast, we do observe qualitative changes in processing dynamics between men and women. Our results suggest that the time-course of visual processing is relatively insensitive to experience, but can be impacted by fundamental physiological differences like those underlying sex. 


\title{
Oculomotor capture by stimuli that signal the availability of reward
}

\author{
Jan Theeuwes ${ }^{1}$, Tom Nissens ${ }^{1,2}$, Mike E. Le Pelley ${ }^{3}$, Michel F. Failing ${ }^{1}$ \\ ${ }^{1}$ VU University Amsterdam, Netherlands \\ 2 Ghent University, Ghent, Belgium \\ ${ }^{3}$ School of Psychology, University of New South Wales, Sydney, Australia \\ J.Theeuwes@vu.nl
}

It is known that selection is influenced by previous experience of rewards. Stimuli whose selection was previously rewarded continue to capture the eyes in a subsequent test session when these rewards are no longer available (Theeuwes \& Belopolsky, 2012). In this study we provide evidence for oculomotor capture by stimuli that merely signal the magnitude reward. The selection of these stimuli was not necessary but instead detrimental for actual payout. Participants to make a saccade to a diamond among 5 uniquely colored circles. The color of one of these circles signaled the magnitude of reward available for that trial. Even though the circle signaling the reward was never part of the task set nor physically salient, it captured the eyes. We conclude that task-irrelevant and non-salient stimuli that signal the availability of reward gain priority in oculomotor selection even if selecting them is not necessary but rather detrimental for reward payout. These findings expand the growing evidence which suggests that attentional selection cannot fully be explained in terms of the traditional separation in top-down and bottom-up processes (Awh, Belopolsky \& Theeuwes, 2012).

\section{The impact of reward on low-level eye movement control Eugene McSorley, Rachel McCloy \\ University of Reading, United Kingdom e.mcsorley@reading.ac.uk}

Eye movement control involved in target selection reflects the evidence supporting a choice but confidence has little impact. Here we examine whether a reward associated with target choice impacts on saccade control. Performance increased and saccade latency decreased with increasing evidence, whereas, unlike previous findings, saccade trajectory deviations were equally away from the non-selected target across all motion coherence levels suggesting that the extent of inhibition did not vary with evidence supporting choice and that the presence of a reward structure overrides effects of evidence. To further examine the effect of reward a second experiment was carried out with two conditions in which the amount of reward associated with an opt-out selection was either low or high. We found similar performance indicators in both reward conditions (number correct increases and latencies decrease as evidence increases) but high reward trials resulted in little trajectory deviation across evidence level. Low reward trials showed curvature away from non-selected target equally across level of supporting evidence. We conclude that the presence of a high-level reward impacts on low-level eye movement control in a manner in which confidence does not and in a way that overrides the impact of the evidence used to support choice. 
Talks

\section{Saccadic Vector Averaging For Auditory-Visual Stimuli \\ Muriel Dysli, Mathias Abegg \\ University hospital Bern, Switzerland \\ muriel.dysli@insel.ch}

\section{Purpose}

When two visual stimuli are displayed in close temporal and spatial relationship, elicited saccades land at an intermediate position between the stimuli. This had been referred to as 'global effect'. Saccadic amplitudes to auditory and combined auditory-visual stimuli are less investigated, even though auditory and visually driven neural activity converges within the saccadic network. We tested whether the global effect is present for visual, auditory, and combined auditory-visual stimuli.

Method

Saccadic amplitudes for auditory, visual, and combined auditory-visual stimuli were assessed with an infrared eyetracking system.

Results

We found that two simultaneously presented visual stimuli elicited saccades with a landing point in between the stimuli. A similar effect was found for only auditory stimuli and for combined visual-auditory stimuli. The landing positions in the latter however strongly depended on the saliency of the visual distractors.

Conclusion

We found a spatial averaging effect between auditory and visually driven saccades indicating that the global effect is not limited to visual stimuli alone. This effect can only be found with low salient visual stimuli, which suggests a predominance of visually driven saccades. These findings support the notion of common processing-pathways of auditory and visual saccades. 


\title{
Scanpaths, saliency, and fixation memory
}

\author{
Room: HS 31
}

\section{Modeling scanpath statistics obtained from mobile eye-tracking Ralf Engbert \\ Universität Potsdam, Germany \\ ralf.engbert@uni-potsdam.de}

With the newest generation of mobile eye-tracking devices, high-precision eye movement data are becoming more and more available for natural tasks. Scanpath prediction for these tasks is more complicated than for static displays due to the continuously changing visual input. Since a number of saliency models have been developed for gaze prediction on time-dependent input, the missing interface for quantitative scanpath prediction is between saliency computations and dynamic models of saccade generation. Recently, we proposed an activation-based mathematical model (Engbert et al., 2015, J Vis) that uses activation dynamics between an attention map and a fixation map for inhibitory tagging to generate realistic spatial statistics for static visual scenes. Here we extend this model to scanpath prediction for mobile eye-tracking. Motivated by the theory of spatial point processes, we evaluate the model using first-order statistics (fixation durations, 2D density of fixations), second-order statistics (spatial correlations), and mark correlations that indicate statistical associations between fixation duration and fixation location. Using large-scale numerical simulations, we show that the model performs much better than random sampling of gaze positions from saliency maps. We conclude that similar principles are underlying gaze control in static and time-dependent scenes.

\section{Investigating scanpaths: The effect of memory}

\author{
Hans A. Trukenbrod \\ University of Potsdam, Germany \\ Hans. Trukenbrod@uni-potsdam.de
}

We need to move our eyes to inspect areas of interest with high visual acuity. During scene perception, fixation locations can be predicted by top-down (e.g., task instruction) and bottom-up factors (e.g., luminance contrast) that are strongly related to image content. In addition, a number of image-independent biases have been reported (e.g., central fixation bias, saccadic momentum). Recently, we demonstrated that the pair correlation function (PCF) reveals aggregration of fixations during single trials (Engbert et al., 2015, J Vis). Resulting fixation clusters cannot be explained by the overall inhomogeneity of fixation locations observed on an image. Here we show that the PCF is sensitive to changes in scanpaths induced by different viewing strategies. We presented images twice to manipulate the top-down effect of memory on scene viewing. Our results replicate the observation of fixation clusters during single trials at short length scales $\left(<3^{\circ}\right)$. The second presentation of an image augmented the effect. We interpret the PCF as a quantitative index for the size of the attentional window, which decreases during the second inspection of images. In general, the limited attentional window reinforces inspection of locations close to the current fixation location. 
Talks

\title{
A scanpath algorithm for dynamic regions of interest and complex task environments
}

\author{
Ryan M. Hope, Wayne D. Gray \\ Rensselaer Polytechnic Institute, United States of America \\ hoper2@rpi.edu
}

In the analysis of eye movements, a lofty goal of many researchers is to deduce from fixation locations what a person is paying attention to at any given moment. Traditionally, the object or region of interest (ROI) that is closest to each fixation is assumed to be the focus of attention. When this process is repeated over a sequence of fixations the resulting pattern is often referred to as a scanpath. Algorithms for calculating scanpaths typically require that ROIs be simple geometric shapes (like circles or rectangles). This restriction has not been much of an issue historically as most tasks used in eye movement research involve static displays of relatively simple stimuli. However, eye movement research in complex tasks (like video games) is becoming ever more popular and traditional scanpath algorithms have no way to handle dynamic ROIs that can move, overlap with one another, change size, change shape or even disappear completely. Here we will discuss many of the challenges associated with calculating scanpaths in dynamic environments as well as introduce a novel scanpath algorithm specifically designed to address the issues discussed.

\section{Comparing saliency maps and human focus maps when free-viewing landscape photographs}

\author{
Lien Dupont, Kristien Ooms, Veerle Van Eetvelde \\ Department of Geography, Ghent University, Belgium \\ lien.dupont@ugent.be
}

In this study, we analyse to which degree the distribution of human fixations on landscape photographs matches with computationally generated predictions based on saliency maps. In addition, we investigate if differences in similarity occur based on the degree of urbanisation present in the landscape photograph. Therefore, an eye-tracking experiment was conducted in which 42 subjects were asked to free-view 74 landscape photographs. The resulting fixation patterns were visualised in greyscale focus maps. Subsequently, these images as well as the predictive saliency maps were transformed into ASCII-matrices containing the actual value (0-255 or white-black) of each pixel. Finally, a pairwise comparison was performed between each pixel of the focus map and its corresponding pixel on the saliency map using the Spearman's rank correlation coefficient. The results indicate a fairly strong positive correlation between the focus maps and the saliency maps. The correlation seems to be higher for weakly urbanised landscapes (few isolated buildings) compared to completely rural scenes (no buildings) and highly urbanised landscapes (dominated by buildings). Thus, especially for the former type of landscape, the viewing pattern can be relatively well predicted. This prediction reliability of the saliency maps could be helpful and time-saving in visual impact assessment procedures. 


\section{Let me tell you where you looked! How much do people know about their own fixations? \\ Melissa Le-Hoa Võ ${ }^{1}$, Jeremy M. Wolfe ${ }^{2}$ \\ ${ }^{1}$ Goethe University Frankfurt, Germany \\ ${ }^{2}$ Brigham and Women's Hospital, Harvard Medical School, Boston, United States of America mlvo@psych.uni-frankfurt.de}

One might end an unsuccessful search for the cat out of the conviction that, "I have looked everywhere!" But how much do we really know about where we looked? We tested participants on an easy change detection task. They looked at pairs of indoor scenes for 3 seconds each, attempting to detect a change between the first and the second scene. On $25 \%$ of trials, participants were asked to indicate, "where you think you just fixated". They responded by making mouse clicks on 12 locations in the unchanged Scene\#1. After 135 trials, observers saw 10 new scenes and were asked to put 12 clicks where "you think someone else would have looked". While observers' located their own fixations more successfully than a random model, their performance was no better than when they were guessing someone else's fixations. This was true even though we queried fixation locations immediately after scene viewing and even though participants performed the task on many scenes. At least for these indoor scenes, our memory for our own fixation locations appears to add nothing beyond what commonsense tells us about where someone else would have looked. 


\title{
Eye-tracking: Issues of measurement
}

Room: HS 32

\author{
iMap 4: An Open Source Toolbox for the Statistical Fixation \\ Mapping of Eye Movement data with Linear Mixed Modeling \\ Junpeng Lao ${ }^{1}$, Sébastien Miellet ${ }^{1,2}$, Cyril Pernet ${ }^{3}$, Nayla Sokhn ${ }^{1}$, Roberto Caldara ${ }^{1}$ \\ ${ }^{1}$ Department of Psychology, University of Fribourg, Fribourg, Switzerland \\ 2 Department of Psychology, Bournemouth University, Bournemouth, United Kingdom \\ ${ }^{3}$ Centre for Clinical Brain Sciences, Neuroimaging Sciences, University of Edinburgh, United Kingdom \\ junpeng.lao@unifr.ch
}

A major challenge in modern eye movement research is to statistically map where observers are looking at. Compared to signals of contemporary neuroscience measures, such as M/EEG and fMRI, eye movement data are sparser with much larger variations across trials and participants. As a result, the implementation of a conventional linear modeling approach on two-dimensional fixation distributions often returns unstable estimations and underpowered results.

Here, we tackled this issue by applying a pixel-wise Linear Mixed Models on the smoothed fixation data with each subject as a random effect. All the possible linear contrasts for the fixed effects (main effects, interactions, etc.) could be performed after the model fitting. Importantly, we introduced a novel spatial cluster test based on bootstrapping to assess the statistical significance of the linear contrasts. This approach is validated by using both experimental and Monte Carlo simulation data.

We implemented this approach in an open source MATLAB toolbox - iMap 4 - with a userfriendly interface providing straightforward, easy to interpret statistical graphical outputs. iMap 4 matches the standards of the robust statistical neuroimaging methods. iMap 4 should thus provide an easy access to robust data-driven analyses for the statistical spatial mapping of eye movement data.

\section{Accurate Pupil Localization Using Contour Sampling for Excimer Laser Eye Surgery Horng-Horng Lin ${ }^{1}$, Kuo-Chih Chiu ${ }^{2}$ \\ 1 Southern Taiwan University of Science and Technology, Taiwan, Republic of China \\ 2 Excelsius Medical Co., Ltd., Taiwan, Republic of China hhlin.tw@gmail.com}

To enhance the precision of corneal curvature correction in eye surgery using excimer laser, we present a new surgical system equipped with an original design of eye stabilizer and an efficient pupil localization module using a near-infrared camera. A novel pupil localization algorithm based on sampling of image contours is implemented in the module to guide the real-time positioning of eye stabilizer on a target eye prior to the laser operation. Experimental results of video-based pupil localization using both real and glass eyes show that the proposed algorithm can track pupil movements precisely, even under several challenging conditions of image blurs, specular reflections, simulated surgical smokes and different lighting conditions. As a result, the proposed algorithm achieves, in average, 1 pixel localization error of pupil centers in the challenging test videos ( 2485 frames in total) with 388 manually-labeled benchmark frames, and outperforms the popular Hough transform-based method for pupil circle detection. Such quantitative assessments of the accuracies of videobased pupil localization for medical applications are rarely seen in previous work, and the presented results may provide a new baseline for future comparisons. 


\title{
The effect of pupil size on the dynamics of the eye tracker signal during saccades
}

\author{
Marcus Nyström ${ }^{1}$, Ignace T. C. Hooge ${ }^{2}$, Richard Andersson ${ }^{1}$ \\ ${ }^{1}$ Lund University, Sweden \\ 2 Utrecht University, Netherlands \\ marcus.nystrom@humlab.lu.se
}

Several papers have recently reported that changes in pupil size introduce drift in the eyetracker signal during fixation. One source of this drift originates from the fact that the shape of the pupil changes along with the diameter, and therefore its center of mass will be displaced even in the absence of an actual eye rotation.

We report a pupil-size dependent change in the dynamics of the eye-tracker signal also during saccades. For instance, data recorded with a large pupil have higher saccade peak velocities and larger post-saccadic oscillations, even though the underlying eye rotation is similar. An extreme example of how pupil size can affect the eye-tracker signal is recorded from an aphakic eye, where the crystalline lens is absent.

We conclude that the pupil size should be considered not only when recording and analyzing data acquired during fixation, but should also be taken into account when investigating saccade dynamics.

\section{Pupil-CR technique is not suitable for studying detailed dynamics of eye movements}

\author{
Ignace T. C. Hooge ${ }^{1}$, Kenneth Holmqvist ${ }^{2}$, Marcus Nyström ${ }^{2}$ \\ ${ }^{1}$ Experimental Psychology, Utrecht University, Netherlands \\ 2 Humanities Laboratory, Lund University, Sweden \\ i.hooge@uu.nl
}

Purpose: Many eye trackers use the corneal reflection (CR) signal to compensate for small movements between the camera and the subject's head. We wonder how the CR and pupil signals contibute to post saccadic oscillations (PSO) observed in the eye tracker signal of high quality video eye trackers. To investigate this question we separately analyzed the pupil and CR signal at the end of a saccade.

Methods: Four subjects conducted saccades between visual targets separated by $5^{\circ}, 10^{\circ}$, $22.5^{\circ}$ and $35^{\circ}$. Saccades were measured binocularly @500Hz.

Results: We observed lower PSO magnitude following lower deceleration at the end of the saccade. This is true for the CR, the pupil and the combined pupil-CR signal. However, the shape of the PSO differs between the CR and the pupil signal. In addition, the first peak of pupil-PSO occurs earlier than the first peak of CR-PSO. The delay between CR and pupil PSO causes complicated PSO shapes in the eye tracker (=pupil-CR) signal.

Conclusion: The pupil-CR technique is not suitable for studying detailed dynamics of eye movements because the pupil and CR signal have different dynamic properties (temporal and spatial). 
Talks

\title{
New Onset Gaze Dependent Disparity in Healthy Humans Changes Saccades, Vergence Eye Movements and Phoria
}

\author{
Mathias Abegg \\ University of Bern, Switzerland \\ mathias.abegg@insel.ch
}

A new onset cranial nerve palsy may cause a change of ocular motility and result in incomitant strabismus, i.e. a viewing direction dependent ocular misalignement. In this study we have investigated the physiological oculomotor response of healthy individuals in conditions of incomitance.

We used a haploscope and prisms to present binocular stimuli with gaze dependent image disparity. We measured saccades, vergence movements and phoria elicited by eye movements toward a gaze position with increased image disparity.

New onset stimulus disparity induces an immediate saccadic disconjugacy, while the following vergence responses show a gradual increase of vergence velocity. Moreover we found a gaze dependent modulation of phoria which was not limited to saccades but was also found in smooth pursuit eye movements.

Repetitive eye movements into a visual field with increased stimulus disparity led to a rapid modification of the oculomotor response involving saccadic amplitude, vergence velocity and the level of phoria. Together this modified oculomotor response enables a prompter restoration of binocular vision in case of a new onset incomitance.

\section{Eye tracking with dogs Soon Young Park \\ Messerli Research Institute, Austria prevetsoon@gmail.com}

For researchers studying cognitive abilities of domestic dogs, eye tracking is an attractive tool to objectively approximate mental focus of the species during perception of scenes in question. There have been several eye tracking studies investigating socio-cognitive abilities of domestic dogs. However, the eye-movement events of the species, which could be species-specific, have not been explored yet. Consequently, the algorithms of event detection used in the studies are far from being in consensus. Clear classification of the eyemovement events based on common event detection algorithms is necessary to enable further discussion and comparisons of the studies among researchers. Because the subjects are household dogs recruited through owners, the eye tracking procedures used in such studies need to avoid invasive techniques. This challenges researchers to come up with strategic procedures guaranteeing stable recordings with reliable calibration results, while still being animal friendly. In this study, tailored dog training to overcome the challenges have been developed to record eye movements of dogs during scene perception. Collected data have been analyzed to identify eye movement events. The experience of dog eye tracking and the results of the event detection analysis will be shared in this talk. 


\title{
Friday, August $21^{\text {st }}, 8.50$ am $-10.30 / 10.50$ am
}

\section{Eye tracking in visual aesthetics: Perception of art}

\author{
Room: HS 7
}

\section{Using a concealed, calibration free eye-tracker for studying art perception in the museum}

\author{
Mario Thalwitzer, Hanna Brinkmann, Raphael Rosenberg \\ University of Vienna, Austria \\ mario.thalwitzer@univie.ac.at
}

Eye-tracking has proved to be a prolific method for studying art perception. However the validity of data in studies concerned with aesthetic appreciation is often questioned due to the context of the laboratory setting. It is claimed that findings of laboratory studies cannot be transferred to real life situations such as beholding paintings in a museum because of the differences in the environment, the modalities of presentation and the aura of the original art work. Hence, a data comparison of an in situ study with laboratory data is a major gap in research of art perception. However, till lately methodological boundaries prevented eye-tracking experiments in museums or resulted in transforming the museum in a laboratory. We conducted a study at the Kunsthistorisches Museum Vienna and used a newly developed calibration-free eye tracker prototype from the Fraunhofer research center Ilmenau. It is based on an advanced approximation of the visual axis (Klefenz et al. 2010). Visitors became aware of being test persons only after they had been recorded. We would like to present the eye tracker system, our study design, the data obtained in the museum and a comparison with data recorded in our laboratory.

\section{Arbitrarily shaped areas of interest based on gaze density gradient \\ Wolfgang Fuhl, Thomas Kübler, Katrin Sippel, Wolfgang Rosenstiel, Enkelejda Kasneci \\ Eberhard Karls Universität Tübingen, Germany wolfgang.fuhl@uni-tuebingen.de}

Studying how people look at paintings usually involves the automated identification of areas that highly attract the user's gaze. Such areas of interest (AOIs) may represent either salient or semantically relevant entities of the painting. While current gaze clustering approaches can successfully identify areas of high gaze density (e.g., via mean-shift as proposed by Santella, A. and DeCarlo, D. (2004)), determining the extension of such areas is non-trivial. Circular and elliptic approximations, which are commonly used for this task, do not fully cover the shape defined by the gaze density at a specific location. We propose two methods for shape identification of AOIs. The first is based on a local maxima intensity dependent threshold, whereas the second on the calculation of gradient direction and runs unparameterized. Both methods use a density map as input which can be computed from gaze points, fixations, or fixation clusters. An implementation of these algorithms will integrated in the next version of Eyetrace (Sippel et al. 2015), a tool for analysis and visualization of eye-tracking data. Eyetrace offers further processing capabilities in order to create and visualize transition matrices based on these clusters. 
Talks

\title{
Absorption, theatricality and modes of spectatorship in the artworks of Edouard Manet
}

\author{
Nick Donnelly ${ }^{1}$, Beth Harland ${ }^{2}$, Simon P. Liversedge ${ }^{1}$ \\ 1 University of Southampton, United Kingdom \\ 2 Lancaster University, United Kingdom \\ n.donnelly@soton.ac.uk
}

Many see something unprecedented in the paintings of Edouard Manet, in particular a radical mode of pictorial address that creates a tension between the absorptive and the theatrical (Fried, 1993). A critical question is whether this distinction has a psychological reality manifest in visuo-cognitive processes. In two recent studies we have explored the visuocognitive correlates of absorption and theatricality in Manet's paintings. In Harland, et al., (2014) we focused on visual inspection of 'A Bar at the Folies Bergere' (Manet, 1882). In contrast to experts, we showed novices to be largely unaware of the geometric and compositional elements contributing to the ambiguity of combining absorption and theatricality. In a second study (Donnelly et al., submitted), we focused on misaligned gaze in sitters in Manet's portraits: gaze being the most obvious cue to either absorption or theatricality. We found novices, art experts and ophthalmic experts to be similarly sensitive to gaze ambiguity. We conclude that novices are sensitive to the absorptive/theatrical distinction but only when supported by readily interpretable socio-cognitive cues such as gaze and gaze ambiguity. In a new study we report on the consequences of the presence of gaze ambiguity for the visuo-cognitive processing of Manet's portraits.

\section{Temporal unfolding of eye movements when looking for beauty and complexity}

\author{
Michael Forster, Marcos Nadal, David Brieber, Helmut Leder \\ University of Vienna, Austria \\ michael.forster@univie.ac.at
}

People often assess their surroundings in terms of beauty, liking or preference. Yet, little is known about what makes this aesthetic way of looking special. Using eye tracking, we aimed to ascertain whether people deploy specific exploratory strategies when they approach visual stimuli with an aesthetic attitude, compared to when they do so with a pragmatic orientation. We wished to ascertain how time constrains these viewing strategies. Participants viewed 60 images and artworks for either 3 s (short condition) or 10s (long condition). In the first block, participants were asked to rate the images' beauty (aesthetic orientation) or their complexity (pragmatic orientation). In the second block, participants were asked to perform the other task, in a counterbalanced fashion. Linear mixed effects models of eye movements in the long condition revealed that in the aesthetic orientation, fixation duration increased, and saccade amplitude decreased over viewing time. When comparing the first $3 \mathrm{~s}$ of the long condition with the short condition, the models showed that, independently of orientation, participants made longer fixations and shorter saccades in the long than in the short condition. Results are discussed in relation to focal and ambient processing modes, and to the time constraints imposed by usual laboratory methodology. 


\title{
Investigating deficit theories of dyslexia with naturalistic reading paradigms using eye-movement recordings
}

Room: HS 30

\section{On forward inferences of fast and slow readers. An eye movement study}

\author{
Stefan Hawelka ${ }^{1}$, Sarah Schuster ${ }^{1}$, Benjamin Gagl ${ }^{2}$, Florian Hutzler ${ }^{1}$ \\ 1 University of Salzburg, Austria \\ 2 Goethe University Frankfurt \\ stefan.hawelka@sbg.ac.at
}

Unimpaired readers process words incredibly fast and hence it was assumed that top-down processing, such as predicting upcoming words, would be too slow to play an appreciable role in reading. This runs counter the major postulate of the predictive coding framework that our brain continually predicts probable upcoming sensory events. This means, it may generate predictions about the probable upcoming word during reading (dubbed forward inferences). Trying to asses these contradictory assumptions, we evaluated the effect of the predictability of words in sentences on eye movement control during silent reading. Participants were a group of fluent (i.e., fast) and a group of speed-impaired (i.e., slow) readers. The findings indicate that fast readers generate forward inferences, whereas speedimpaired readers do so to a reduced extent - indicating a significant role of predictive coding for fluent reading.

\section{Eye movements and parafoveal processing in children with dyslexia}

\author{
Julie A. Kirkby, Rhiannon S. Barrington, Lucy C. Worf, Abby E. Laishley \\ Bournemouth University, United Kingdom \\ jkirkby@bournemouth.ac.uk
}

A key characteristic of skilled reading is the ability to pre-process words before directly fixating them. Well documented preview benefit effects indicate that both letter identity and letter order facilitate early stages of visual word recognition for orthographically related previews (Johnson, Perea, \& Rayner, 2007). We understand virtually nothing, however, about parafoveal pre-processing for children with dyslexia. A boundary paradigm was used as single line sentences were silently read and included parafoveal previews of target words that were either (1) identical to the target (e.g. school) (2) a transposed-letter neighbour (e.g. cshool) or (3) a substituted-letter non-word (e.g. uohool). We monitored the eye movements of three participant groups - children with developmental dyslexia, typically developing children matched for chronological age and typically developing children matched for reading age. The results showed disruption during reading from non-identical previews, but less disruption from transposed-letter previews compared to substituted-letter previews. This pattern of results held for all reading ability groups. Therefore, the results show that children with dyslexia gain both letter position and letter identity information from the parafovea during sentence reading. These findings have important implications for theories of developmental dyslexia. 
Talks

\section{A similar correction mechanism in slow and fluent readers after suboptimal landing positions. \\ Benjamin Gagl ${ }^{1,2}$, Stefan Hawelka ${ }^{2}$, Florian Hutzler ${ }^{2}$ \\ ${ }^{1}$ Department of Psychology, Goethe University Frankfurt am Main, Germany \\ ${ }^{2}$ Centre for Cognitive Neuroscience, University of Salzburg, Austria \\ gag $\mid$ @psych.uni-frankfurt.de}

The present eye movement study investigated the optimal viewing position and invertedoptimal viewing position effects in slow readers. The basis of these effects is a phenomenon called corrective re-fixations, which describes a short saccade from a suboptimal landing position (word beginning or end) to the center of the word. The present study found corrective re-fixations in slow readers, which was evident from the inverted-optimal viewing position effects in first fixation durations, the optimal viewing position effect in number of fixations and the in optimal viewing position effect in re-fixation probability. The main result is that slow readers, despite being characterized by a fragmented eye movement pattern during reading, nevertheless share an intact mechanism for performing corrective re-fixations. This correction mechanism is not linked to linguistic processing, but to visual and oculomotor processes, which suggests the integrity of oculomotor and visual processes in slow readers.

\section{Effects of Letter Spacing on Eye Movements during Silent Reading}

Sebastian Peter Korinth, Kerstin Gerstenberger, Christian Jens Fiebach

University of Frankfurt, Germany

korinth@psych.uni-frankfurt.de

Assuming a dyslexia-specific visual crowding deficit, it was previously reported that oral reading performance improved for dyslexic children but not for regular readers when between-letter spacing was enlarged. The present study examined (a) whether wider letter spacing improves reading performance also for non-impaired adults during silent reading, and (b) the association between letter spacing effects and crowding sensitivity. We recorded eye-movements while 24 German students silently read texts presented either with normal or enlarged (2.5pts on font size 12) letter spacing. Two additional tests estimated foveal and parafoveal crowding sensitivity. Word- and participant-specific effects of extra-letterspacing were estimated using linear-mixed-effect models. Wider spacing reduced fixation durations for all participants, with slower readers showing stronger effects. Gaze durations in the wide-space condition were shorter for slow but longer for faster readers. However, wider letter spacing also increased fixation probabilities and fixation count with stronger effects for faster readers. Neither foveal nor parafoveal crowding sensitivity correlated with the observed letter-spacing effects. In conclusion, wide letter spacing can reduce word processing time in normally-developed readers during silent reading. This effect is thus not dyslexia-specific, but rather word- and reader-specific. Our data provide no support for the visual crowding hypothesis of spacing effects. 


\title{
Brain activation of slow and fluent readers during natural reading: $A$ fixation-related fMRI study
}

\author{
Fabio Richlan, Sarah Schuster, Stefan Hawelka, Florian Hutzler \\ Centre for Cognitive Neuroscience, University of Salzburg, Austria \\ fabio.richlan@sbg.ac.at
}

We used co-registration of eye movements and functional magnetic resonance imaging (fMRI) to investigate the brain activation of slow and fluent readers during natural, selfpaced, silent sentence reading. Sentence reading compared with fixation baseline resulted in activation of the typical reading network including largely left hemisphere occipital, parietal, temporal, and frontal language regions. In order to identify the neural correlates of slow and fluent reading, we used reading fluency (i.e., words per minute) as a continuous regressor in the statistical analysis. More fluent reading was associated with higher activation in bilateral calcarine, lingual, and superior parietal regions and left posterior middle temporal gyrus. Conversely, slower reading was associated with higher activation in medial superior frontal cortex, left thalamus, and right callosal white matter. To conclude, more fluent readers rely more on regions related to visual-attentional and lexico-semantic processes, whereas slower readers recruit regions of the cingulo-opercular control network required for stable maintenance of goal-directed behavior. The present findings shed light on the brain regions associated with slow and fluent reading during an ecologically valid reading situation. Further analyses will focus on the influence of reading fluency on the neural correlates of word length, frequency, and predictability effects during natural reading. 
Talks

\title{
Eye movements: Clinical
}

Room: HS 31

\section{Attentional Capture by Emotional Scenes across Episodes in Bipolar Disorder: Evidence from a Free-Viewing Task}

\author{
Ana García-Blanco ${ }^{1,2}$, Ladislao Salmerón ${ }^{1}$, Manuel Perea ${ }^{1}$ \\ 1 Universitat de València, Spain \\ 2 Health Research Institute La Fe, Valencia, Spain \\ Ana.Garcia-Blanco@uv.es
}

We examined whether the initial orienting, subsequent engagement, and overall allocation of attention are determined exogenously (i.e., by the affective valence of the stimulus) or endogenously (i.e., by the participant's mood) in the manic, depressive and euthymic episodes of bipolar disorder (BD). Participants were asked to compare the affective valence of two pictures (happy/threatening/neutral [emotional] vs. neutral [control]) while their eye movements were recorded in a free-viewing task. Results revealed that the initial orienting was exogenously captured by emotional images relative to control images. Importantly, engagement and overall allocation were endogenously captured by threatening images relative to neutral images in BD patients, regardless of their episode-this effect did not occur in a group of healthy controls. The threat-related bias in BD, which occurs even at the early stages of information processing (i.e. attentional engagement), may reflect a vulnerability marker.

\section{Hypervigilance for faces but typical gaze following in social anxiety Nicola Jean Gregory, Helen Bolderston, Jastine Antolin

A hypervigilance-avoidance theory has been proposed to explain early attentional biases towards faces followed by their subsequent avoidance, in social anxiety (SA) (Mogg et al., 1997). This theory has arisen from data obtained from tasks presenting static images which lack in ecological validity. Moreover, whilst the gaze of others is considered a threatening cue in SA and is typically avoided, the gaze following mechanism has not been examined in SA. We analysed eye movements, including gaze following in 39 participants, who were either high or low in SA (HSA and LSA respectively) as they watched an emotionally-neutral dynamic social scene. Contrary to the hypervigilance-avoidance hypothesis, whilst HSA participants directed significantly more early fixations towards the face than the LSA group, both groups fixated the face equally after this time. There were no group differences in gaze following. Results suggest that when viewing a neutral social scene, HSA individuals are initially biased towards the face but when threat is not perceived a typical viewing strategy is adopted. Furthermore, although direct gaze may be avoided in SA, the gaze following mechanism may be so automated that it is impervious to higher level socio-cognitive factors such as fear of social evaluation. 


\title{
The Relationship Between Implicit Expectations About Character Behaviour and Eating Disorder Tendencies in Males and Females: Evidence From Eye Movements During Reading
}

\author{
Christina Ralph-Nearman, Ruth Filik \\ University of Nottingham, United Kingdom \\ Lpxcr4@nottingham.ac.uk
}

Eating disorders have a higher mortality rate than all other mental illnesses combined. Current theories debate whether it is body-, food-, or perfectionism-related attentional biases which underlie the development and maintenance of eating disorder behaviour. We used eye-tracking during reading to investigate whether participants' implicit expectations regarding how characters will behave in body-related, food-related, or perfectionism-related scenarios will be related to their tendency towards disordered eating behaviour. In three experiments, participants had their eye movements monitored while they read body-, food-, or perfectionism-related scenarios, which ended with a target sentence containing a critical emotion-based word that either 'matched' or 'mismatched' with one's expectations concerning how the character might react. In Experiment 1, 90 females read about third-person female characters, in Experiment 2, 66 females read a first-person version, and in Experiment 3, 42 males read a third-person male version of the materials, before completing the Eating Disorder Examination Questionnaire (EDE-Q 6.0). Participants' scores on the EDEQ 6.0 were predicted by the size of the mismatch effect for perfectionism-related materials for third-person females, and by food-related materials for first-person females and thirdperson males. Results support theories which propose that perfectionism and food-related biases may be key aspects of eating disorder etiology.

\section{Sensorimotor outward adaptation in schizophrenia}

\author{
Rebekka Lencer, Annegret Meermeier, Karen Silling, Svenja Gremmler, Markus Lappe \\ University of Muenster, Germany \\ rebekka.lencer@ukmuenster.de
}

Background: Schizophrenia is characterized by cognitive deficits associated with disturbances in sensory information processing and motor control. We investigated outward saccadic adaptation and adaptation-induced mislocalization in patients with schizophrenia.

Methods: 14 patients (mean age 37.1 years) and 13 healthy controls (35.1 years) were assessed on a no-gap, no-overlap adaptation task (200 trials): $10^{\circ}$ steps plus $4^{\circ}$ outward target shift immediately after saccade start. In 40 localization trials, run before and after saccade adaptation trials, subjects had to localize a stimulus that was flashed $50 \mathrm{~ms}$ after saccade target onset. Eye movements were recorded at $1000 \mathrm{~Hz}$.

Results: Pre-adaptation saccade metrics did not differ between groups. During adaptation, saccadic amplitudes increased significantly in both groups (controls: $\mathrm{p}<0.001$; patients: $\mathrm{p}$ $<0.05)$. Overall adaptation strength, i.e. mean percent amplitude change of the last 20 adaptation trials compared to pre-adaptation trials, did not differ between groups. Adaptation velocity obtained from exponential fits of the individual data, however, was lower in patients than in controls $(\mathrm{p}<0.05)$. Adaptation-induced mislocalization was similar in both groups.

Conclusion: Although adaptation was slower in patients than controls, the use of error signals for internal recalibration of sensorimotor systems seems to be basically unimpaired in schizophrenia. 
Talks

\title{
Eye Movements indicate no immediate detection of Social Oddities in Complex Scenes in Autism Spectrum Disorder
}

\author{
Valerie Benson ${ }^{1}$, Monica S. Castelhano ${ }^{2}$, Philippa Lucy Howard ${ }^{1}$, Nida Latif ${ }^{2}$, Keith Rayner ${ }^{3}$ \\ 1 University of Southampton, United Kingdom \\ 2 Queen's University, Kingston, Canada \\ 3 University of California, San Diego, United States of America \\ vb1@soton.ac.uk
}

Autistic Spectrum Disorder (ASD) and Typically Developed (TD) adult participants viewed single scenes as their eyes were monitored. The task was to make a value judgement as to whether each scene was in some way 'odd'. All scenes contained people, with one category of 'oddness' operationally defined as perceptual, and another category defined as social. There were no group differences in accuracy, but the ASD group were slower overall to respond manually. Eye movements revealed that the ASD group made more fixations overall and more refixations into the target regions of the pictures, repeatedly inspecting these areas prior to responding. Additionally, as indexed by the first fixation duration into the target region, and in contrast to the TD group, the ASD group failed to 'pick up' immediately on what was 'odd' for the social violations. Immediate detection of perceptual violations was present in both groups. The findings suggest that it is not just the presence of people per se that results in processing deficits in ASD in scene perception. Rather, it is the nature of the violation that impairs initial detection of oddities in scenes containing people in ASD.

\section{Repetitive Vergence Training in Binocularly Normal Controls \\ Tara Lynn Alvarez, Henry Talasan \\ New Jersey Institute of Technology, United States of America tara.I.alvarez@njit.edu}

Currently, it is not well known whether significant changes would be observed in vergence eye movements before compared to after repetitive vergence eye movement training in those with normal binocular vision. Ten subjects participated in a vergence training experiment designed to minimize the influence of the phoria on the disparity vergence system. The following parameters were quantified: vergence response latency, time to peak velocity, settling time, peak velocity, and accuracy. Accuracy was defined as the difference between the stimulus target and response amplitude measured within a phase plot. For all subjects studied, the following were observed when comparing the results after vergence training to the baseline measurements: 1) near point of convergence and near dissociated phoria did not significantly change $(\mathrm{p}>0.1) ; 2)$ latency, settling timing, and time to peak velocity significantly decreased $(\mathrm{p}<0.001)$; and 3 ) accuracy significantly improved meaning the responses were more likely to be closer to the visual stimulus $(\mathrm{p}=0.02)$. Results support that vergence peak velocity is dependent on the subject's phoria level. The accuracy and temporal properties of vergence eye movement responses can be improved after vergence eye movement training. Results have clinical implications in introducing methods that can be used to asses patients with binocular dysfunction. 


\title{
Eye movements: Applied questions
}

Room: HS 32

\author{
Gaze interaction with textual user interfaces \\ John Paulin Hansen ${ }^{1}$, Haakon Lund ${ }^{2}$, Janus Ask $\varnothing$ Madsen $^{1}$, Morten Jonassen ${ }^{1}$ \\ ${ }^{1}$ ITU, Denmark \\ 2 IVA, Denmark \\ paulin@itu.dk
}

This presentation suggests using rapid serial visual presentation (RSVP) of single words for prompting command options that may be executed by gaze-strokes. In a study with 27 participants the RSVP commands would engage a near-by display; adjust the speed of word presentation; and provide a "back" option for text navigation. People readily understood how to execute RSVP command prompts and a majority of them preferred gaze input to a pen pointer.

We present the concept of a smartwatch that can track eye movements and mediate command options whenever in proximity of intelligent devices that it connects with, i.e. a GazeWatch. For instance, standing next to a monitor, it may suggest to turn it on, if you look up at the monitor now. Command suggestions are provided in the RSVP-format, but they only stay active for a limited time, in which the gaze should be moved to apply them.

\section{Eye Typing for speed: Comparing three different eye typing keyboard layouts}

\author{
Markus Joos ${ }^{1}$, Daniel Liebscher ${ }^{1}$, Wolfgang Rüger ${ }^{1}$, Hans Böhme ${ }^{2}$ \\ 1 Interactive Minds Research \\ 2 University of Applied Sciences Dresden \\ joos@interactive-minds.com
}

In the present study we compared three different eye typing keyboard layouts for performance (Soukoreff , 2010) and subjectively rated differences. All three layouts were identically designed with respect to input parameters, feedback methods, etc. The first (and also base layout for all) was a traditional QWERTZ layout, the second an enhanced base version with an additional horizontal row on top with three word prediction keys and the third a base layout with a novel overlay approach in which the single, most likely prediction was presented on top of the key button users were gazing at. 12 participants ( 9 male, 3 female) took part in the study. An LC Technolgies Eyegaze Edge system was used for gaze input running at $60 \mathrm{~Hz}$. Results revealed that the novel on key prediction layout significantly outperformed both, the traditional QWERTZ keyboard as well as the QWERTZ keyboard with separate prediction buttons. Similarly the input error rate was reduced for the new overlay approach.The same picture also emerged for subjective user ratings of the three different layouts with respect to layout preferences and stress levels. 
Talks

\title{
Gaze-contingent techniques increase infant attention during ERP studies
}

\author{
Estefania Dominguez Martinez ${ }^{1,2}$, Vincent Reid ${ }^{1}$, Eugenio Parise ${ }^{1}$, Tommy Strandvall ${ }^{2}$ \\ ${ }^{1}$ Lancaster University, United Kingdom \\ 2 Tobii Technology AB \\ estefania.dominguez@tobii.com
}

Gaze-contingent paradigms have been proven to be useful during infant attentional training (Wass, Porayska-Pomsta, \& Johnson, 2011) and infant behavioral training (Wang et al., 2012). In this study, we assessed whether the use of gaze contingency during an event related potential (ERP) study improved the attention - and therefore the morphological quality of the ERPs - of 12-month-old infants. Two conditions were compared; in the gaze-contingent condition infants had to successfully fixate on a fixation image prior to the stimuli image to trigger the next trial. In the non-gaze contingent condition a standard ERP methodology was used to trigger the trial. The results showed that the infants assigned to the gaze-contingent condition attended to the stimuli for more trials. They also looked at the critical visual component of the stimulus for more trials than the infants assigned to the non-gaze-contingent condition. This was also revealed in the ERP component that is related to attention in infants, the Nc, which was significantly more negative in amplitude for infants assigned to the gaze-contingent condition. This result indicates that the infants allocated more attention to the stimuli -. Gaze-contingent paradigms clearly facilitate infant visual ERP research.

\section{A closer look at the differences in eye movement between successful and unsuccessful solvers while solving geometry problems with handwriting devices}

\author{
John J. H. Lin, Sunny S. J. Lin \\ National Chiao Tung University, Taiwan, Republic of China \\ john.jrhunglin@gmail.com
}

We have connected a handwriting-device to eye-tracker to study eye movement differences between successful and unsuccessful solvers of geometry problems (Lin \& Lin, 2013, 2014). The previously used problems, each presented in a screen showing a pair of similar-triangles with multiple information, were sliced and given screen by screen with the participants' push-button request in this study. The problem was given from the diagram-empty, the angle symbols on the diagram as well as the length quantities. Incrementally providing information makes it possible to observe the processes with high time resolution. We also integrated solvers' task performance, cognitive load, and a questionnaire as a probe to investigate the source of difficulties. Sixty-two high school students participated in this study. The results indicated fixation-based measures were more sensitive than saccade-based ones in distinguishing successful and unsuccessful solvers. Unsuccessful solvers spent more time fixating on diagrams to extract information. Solvers perceived considerable difficulties in the integrating phase of problem-solving while reading diagram and simultaneously rotating it. 


\title{
The Gaze Controlled Game as a Cognitive Training for Children with Math Difficulties
}

\author{
Jacek Matulewski ${ }^{1,2}$, Bibianna Balaj ${ }^{2}$, Matgorzata Gut ${ }^{2}$, tukasz Goraczewski ${ }^{2}$, Joanna \\ Dreszer $^{2}$, Jakub Majewski ${ }^{3}$, Ewelina Bendlin ${ }^{3}$, Piotr Cholewa ${ }^{3}$, Alicja Majka ${ }^{3}$, Maciej \\ Kowalski $^{4}$ \\ ${ }^{1}$ Institute of Physics, Faculty of Physics, Astronomy and Informatics, Nicolaus Copernicus University, \\ Grudziadzka 5, 87-100 Torun, Poland \\ 2 Interdisciplinary Centre for Modern Technologies, Nicolaus Copernicus University, Poland \\ 3 informatics students, Faculty of Physics, Astronomy and Informatics, Nicolaus Copernicus University, \\ Grudziadzka 5, 87-100 Torun, Poland \\ ${ }^{4}$ high school student, IV Liceum Ogólnokształcace im. Tadeusza Kościuszki \\ jacek@phys.uni.torun.pl
}

Dyscalculia is a disorder of the basic arithmetic skills. The screening test of the dyscalculia risk for children at the preschool age, although it cannot be basis for the final diagnosis, is very useful. It makes the early intervention possible and lengthen the time of possible training. In this perspective the computer trainings are particularly interesting. Unfortunately the "math training", even the computer one, cause the child's strong aversion.

Our proposal is to transform the computer training into an real action game i.e. introduce the gamification, modern technologies of graphics and new game controllers, including the eye-tracker. We expected that such game will increase the motivation and perseverance during the training making it more effective.

We prepared the training game, which develops the mental numerical axis, as well as the computer screening test of dyscalculia hazard. Both program use eyetrackers. Using our test and the standardized test of dyscalculia risk we identified two sets of 15 pupils, having mathematical difficulties and the control group. In the second stage both groups carried out the game training. We observe the improvement of the specific math skills in both groups. Additionally, we compare the eye-movement of children in both groups. 


\section{Posters}




\title{
Monday, August $17^{\text {th }}, 3.00 \mathrm{pm}-5.00 \mathrm{pm}$
}

\section{Cognitive influences}

\author{
Assessment of Cognitive Functions using Eye Tracking \\ Sabine Heuer, Melissa Pinke \\ University of Wisconsin Milwaukee, United States of America \\ heuer@uwm.edu
}

\begin{abstract}
Aphasia is the impairment of language comprehension and production due to brain injury. Nonlinguistic deficits in cognitive flexibility contribute to impaired turn taking, topic maintenance, and communication repair in people with aphasia. Nonlinguistic switching tasks index cognitive flexibility. In the single task the matching criterion remains the same across trials - cognitive switching demands are low. In the mixed task the search criterion switches unpredictably -cognitive demands are high due the need to monitor multiple competing tasks. Eye-tracking has excellent potential to assess cognitive flexibility in aphasia.

The purpose was to develop a novel eye-tracking switching task and to determine the sensitivity to increases in cognitive demands between single and mixed task conditions.

Eye movements of 20 language-normal participants were recorded as they looked at a computer screen and completed the experimental single and mixed switching task. Latency of First Fixation (LFF), First Pass Gaze Duration (FPGD), and First Fixation Duration (FFD) were computed across all trials. The difference between single and mixed conditions was calculated (switching cost). Paired-samples t-tests revealed significant switching cost for LFF and FPGD.

The eye-movement method indexed increased cognitive demands associated with maintaining multiple task sets in the mixed-compared to the single-task condition.
\end{abstract}

\section{The mechanism of the Einstellung (set) effect: An investigation with eye tracking

\author{
Mario Graf, Anna Conci, Merim Bilalić \\ Alps Adria University of Klagenfurt, Austria \\ mario.graf@aau.at
}

The Einstellung (set) effect is a well known phenomenon where an idea that comes immediately to mind prevents other alternatives from being considered. In the water-jar experiment by Luchins (1942), participants were given a series of problems that could be solved by a fixed method that they quickly learned. However, many could not solve an apparently similar problem for which the fixed method did not work. The Einstellung effect has been many times replicated, but the cause behind it is still unknown. Here we illuminate its mechanism by recording eye movements during the solving of the water-jug problems. We demonstrate that the participants experience the Einstellung effect because they fixate the elements that were helpful in the previous problems but are not relevant in the new problem. The participants who eventually find the right solution, slowly disengage their attention, as measured by eye fixations, from the irrelevant elements and start devoting it to the relevant elements. Our eye movement study demonstrates that the first idea that comes to mind directs our attentions towards the aspects that are relevant to itself and away from those that are not. The Einstellung situations illustrate a drawback of this otherwise efficient mechanism. 


\title{
Diverging Thoughts, Diverging Eyes?
}

\author{
Sonja Walcher, Christof Körner, Mathias Benedek \\ University of Graz, Austria \\ sowalcher@gmail.com
}

This study investigated which eye parameters are indicative of perceptual decoupling during states of goal-directed internally-focused attention. Earlier reports had suggested that eye parameters are sensitive to spontaneous periods of mind-wandering. Our study examined eye behavior during tasks which require that attention was deliberately focused on internal processes. States of internally-directed and externally-directed attention were induced in a sample of 49 participants using a divergent thinking task (i.e., creative idea generation) and a working memory task that required constant monitoring of external events (i.e., $\mathrm{N}$-back task), respectively. We analyzed different eye parameters, including eye vergence which was measured by the horizontal fixation difference between eyes and which is considered an indicator of the distance of visual focus. We found that perceptual decoupling during internally-directed attention was accompanied by higher stimulus-independent variability in both pupil diameter and eye vergence. However, lower stimulus-independent variability in eye vergence was associated with better performance during creative idea generation. This finding suggests that performance of internal attention tasks is rather supported by an effective maintenance of visual disengagement. Our results further point to individual differences in the ability to achieve stable states of stimuli-independent eye vergence.

\section{Eye movements as predictors of users' cognitive load with parallel coordinate plots}

\author{
Stefanie Wetzel, Sven Bertel \\ Bauhaus-Universität Weimar, Germany \\ stefanie.wetzel@uni-weimar.de
}

We report on results of a study in which users' eye movements were captured during a visual reasoning task in order to assess cognitive load. We designed four different variants of interactive parallel coordinate plots in order to induce different load levels. Parallel coordinate plots are a common method of visualising multivariate multidimensional data; zooming and brushing operations permit detailed data exploration. In our study, NASATLX scores served as a cognitive load baseline measure. Pupil size, blink rate, and transitions between constituent diagram components were registered and combined as useful predictors into a linear regression model of the baseline score. Further eye movement metrics, such as fixation rates and saccade amplitudes, and selected individual measures of working memory and perceptual speed, and of expertise with parallel coordinate displays, did not successfully predict cognitive load during the tasks. In this contribution, we reflect on method and methodology of analysis, as well as on the study design, and derive recommendations for further analyses of cognitive load via users' eye movements with interactive visualisations. 
Posters

\author{
How I speak is what I see - About differences in gaze behaviour \\ between Korean and German speakers \\ Florian Goller $^{1}$, Donghoon Lee ${ }^{2}$, Ulrich Ansorge ${ }^{1}$, Soonja Choi ${ }^{1,3}$ \\ ${ }^{1}$ University of Vienna, Austria \\ 2 Pusan National University \\ 3 San Diego State University \\ florian.goller@univie.ac.at
}

Languages differ in how they categorize spatial relations: While German, as English, differentiates between containment (in) and support (auf/on) with distinct spatial words (a) den Kuli IN die Kappe ('put pen in cap'); (b) die Kappe AUF den Kuli stecken ('put cap on pen'), Korean uses a single spatial word (KKITA) collapsing across (a) and (b) into one semantic category particularly when the spatial enclosure is tight-fit. Korean uses a different word (NEHTA) for loose-fits (e.g., apple in bowl). But how do these differences guide the attention of the speaker?

In a cross-cultural study, we compared native German speakers with native Korean speakers. Participants rated the similarity of two successive video clips of several scenes where two objects were joined or nested (both in a tight or loose manner). Throughout the experiment, participants' eye movements were measured.

The data showed that Korean speakers distinguished much more between tight and loose relations than German speakers. Additionally, Korean speakers also fixated more on the region where the two objects touched than German speakers. The data of this pilot study is discussed in the light of linguistic and psychological theories of attention.

\title{
Seeing difficulty in the eyes - metacognitive fluency and eye movements

\author{
Nina Chrobot ${ }^{1,2}$, Norbert Schwarz ${ }^{2}$, Tommy Strandvall ${ }^{3}$, Andrzej Falkowski ${ }^{1}$ \\ 1 University of Social Sciences and Humanities, Poland \\ 2 University of Southern California \\ 3 Tobii AB \\ ninachrobot@gmail.com
}

At each step of information processing people receive experiential feedback in form of metacognitive experiences of ease (fluency) and difficulty (disfluency). Consumers use these experiences as information. As a result, metacognitive experiences elicited by information processing may be misread as bearing on the product one reads about (Alter \& Oppenheimer, 2009; Schwarz, 2015). So far fluency has been mostly measured by self-reports. We propose eye tracking as an objective measure of fluency. In a study, we manipulated fonts (easy vs. difficult to read) to alter the levels of cognitive fluency. Participants $(n=143)$ viewed a product web page and estimated the product's price and their willingness to pay (WTP). We recorded participants' eye movements and found that subjects in the 'difficultto-read' group had longer fixation durations, suggesting difficulty in decoding information. Disfluency was also associated with more complex gaze plots. We observed greater switches between areas of interests as measured by transition matrices in the 'difficult-to-read' group. Additionally, our results indicate a link between cognitive fluency and people's judgments, as a disfluent experience had a negative impact on price estimation and WTP. Our study supports the use of eye tracking as proposed objective measure of metacognitive experiences of fluency. 


\title{
From thinking to speaking: (Eye)tracking the time course of sentence formulation
}

\author{
Erica dos Santos Rodrigues, Renê Forster, Jéssica Barcellos \\ PUC-Rio, Brazil \\ ericasr@puc-rio.br
}

It is a point of debate whether grammatical encoding is incremental in relation to message encoding (Gleitman et al., 2007) or whether the former starts only after the planning of a rudimentary message (Griffin \& Bock, 2000). We explored this topic by means of two eyetracker scene description experiments. In Experiment 1 scenes were described while in view. In Experiment 2, an attention-capture resource was added to draw attention to a particular character (agent or patient). Results of both experiments revealed a preference for active structures. No relationship between fixations in the initial $300 \mathrm{~ms}$. and syntactic structure (active/passive) was observed. In the second experiment, however, there was an increase in the number of passives $(p<.02)$ in the patient condition. The absence of correlation between initial fixations and syntactic structure in both experiments suggests that grammatical encoding starts after initial scene apprehension. It seems that the attention-capture has a posterior effect, favoring a particular interpretation of the scene. The favored interpretation of the scene may be occasionally reflected in the linguistic structure, but with no strict relation between initial fixations and chosen subject. Instead, sentence formulation seemed to be guided by cost related issues, since active structures were preferred.

\section{Experimental Methods in Cognitive Linguistics: Cognitive Simulations as Instances of Mental Imagery During Fictive Motion Processing, an Eye-Tracking Study \\ Alena Holubcova ${ }^{1,2}$ \\ ${ }^{1}$ Masaryk University, Czech Republic \\ 2 Humanities Laboratory, Lund University alenaholubcova@centrum.cz}

Available evidence suggests that fictive motion (sentences like "The road is going through the desert") elicits cognitive simulation of motion in our minds. As a consequence, it takes people longer to decide about a fictive motion statement after perceiving a story dealing with long travel distance, difficult terrain or slow travel time; than about a story with short travel distance, easy terrain and fast travel time.

In the current experiment, native speakers of English listen to pre-recorded stories with short and long distance scenarios, while their spontaneous eye movements are being recorded (blank screen paradigm). Each participant then decides about the congruity of a fictive motion statement with the story, and response times of decisions and eye movements during decisions are recorded.

The spatial distribution of all recorded eye movements is compared to the respective scores of the participants on the Object-Spatial Imagery and Verbal Questionnaire. Eye movements during decisions are compared within-participants and across participants.

Moreover, the statements are constructed to reflect instances of summary/sequential scanning (Langacker), and static/dynamic construal (Talmy); and thus disentangle the effect of each on fictive motion processing. The results have the potential to inform the theory of dynamic construal in cognitive linguistics. 
Posters

\title{
The "visual number world": a new approach to study mental arithmetic
}

\author{
Matthias Hartmann, Martin H. Fischer \\ University of Potsdam, Germany \\ hartmann.psychology@gmail.com
}

In language research, the "visual world" is a well-established paradigm to study the time course of language comprehension. In this paradigm, different objects are presented on the screen while listening to spoken sentences involving these objects. We adopted this paradigm to study mental arithmetic: instead of objects, numbers were presented at different locations on the screen, and participants listened to arithmetic problems (e.g. $4+7$ ). We found the highest probability of looking at a particular number around $600 \mathrm{~ms}$ after the onset of the corresponding spoken number, showing that participants look at the numbers they currently process. We also found a systematic reactivation of the first operand after the onset of the second operand. These patterns were subtly influenced by the visual arrangement of the numbers (ascending or descending order from left to right). Particularly, participants were faster to anticipate the solution for a subtraction problem when numbers were arranged in a descending order. This effect was more pronounced for participants showing a stronger spatial-numerical association as assessed by a classical SNARC task. This research demonstrates how eye movements can be used to better understand how a person solves an arithmetic problem.

\section{The role of intention in gaze following \\ Ross G. Macdonald, Maria Staudte \\ Saarland University, Germany rossgmacdo@gmail.com}

Shared intention can affect visual behaviour; in tasks using a cursor to represent gaze, participants align their gaze more when they believe the cursor represents the gaze of a collaborator. Unlike artificial cursors, gaze cues are compulsively followed. Therefore it is unclear whether shared intention would have any effect on our response to these cues. We investigated this issue with a desk-mounted eye-tracking paradigm. Participants viewed videos of a woman gazing at two of eight objects on a tabletop, while a male voice referred to two objects. We manipulated whether or not the verbal and gaze cues were congruent with each other. Crucially, prior to the onset of the experiment, we informed the participants that the woman was either listening to the same sentences and carrying out the same memory task (shared intention), or listening to different sentences and carrying out a different task (distinct intention). Data collection is on-going. We hypothesise that more gaze following will occur in the shared intention condition and that this will manifest in earlier saccades to and longer fixations on gazed-at objects. Regardless of the outcome, our results will contribute to a greater understanding of the role of a gazer's intentions in gaze following. 


\title{
Give me a cue: Effects of visual complexity and description ambiguity on attention
}

\author{
Tim van Marlen ${ }^{1,2}$, Margot van Wermeskerken ${ }^{1,2}$, Tamara van Gog ${ }^{1,2}$ \\ 1 Department of Pedagogical and Educational Sciences, Utrecht University, The Netherlands \\ 2 Institute of Psychology, Erasmus University Rotterdam, The Netherlands \\ vanmarlen@fsw.eur.nl
}

Eye movement modelling examples (EMME) provide students with an expert's demonstration and verbal description of how to complete a task, with the expert's eye movements overlaid on the task. This is expected to guide students' attention and improve their learning outcomes. Support for this hypothesis is mixed, which might result from differences among studies in the visual complexity or the ambiguity of verbal descriptions of the tasks being modeled. Therefore, we explored to what extent visual complexity and description ambiguity influence performance on perceptual identification tasks, in order to establish conditions under which visual cueing (e.g., EMME) might be most beneficial. In Experiment 1 (online) and Experiment 2 (lab) participants viewed images showing 12 cartoon-like characters. They had to correctly identify a character based on a verbal description. Visual complexity (low/high) and description ambiguity (low/high) were manipulated withinsubjects. Both experiments showed lower performance and higher response times with high ambiguity descriptions as compared to low ambiguity descriptions, but no effects of visual complexity. An eye-tracking follow-up study is currently being conducted to test the assumption that description ambiguity influences the time course of visual attention allocation, which could help explain under which conditions EMME will be most effective.

\section{Effect of Exogenous Factors on Eye Movement Based Automatic User Identification}

\author{
Yannik T. H. Schelske ${ }^{1}$, Tandra Ghose ${ }^{2}$ \\ 1 Department of Computer Science, University of Kaiserslautern, Germany \\ 2 Department of Psychology, University of Kaiserslautern, Germany \\ y_schels@cs.uni-kl.de
}

We investigated factors that influence automatic eye-movement (EM) based useridentification. EM arise from endogenous (intrinsic/top-down) and exogenous (extrinsic/bottom-up) factors. User-identification relies on individual differences in endogenous-factors that get suppressed by stimulus/exogenous-factors, thereby reducing user-discriminability. For improving identification performance it is essential to identify endogenous-factors that are robust to suppression by exogenous-factors.

We used perceptual-grouping for manipulating the strength of exogenous-factors. EM of 8 participants were recorded for 10 s each, for 120 trials while inspecting Gabor-fields. The task was to indicate by key-press when red-dot appears anywhere on screen (randomly after $10-13$ s of stimulus-onset). Stimuli contained either no-groups, groups induced by increased luminance or by aligning orientation of a third of Gabor-elements. Detectability of groups was matched. User-identification accuracy of a support vector machine (25 EM -features) was worst (56\%) for no-groups, $63 \%$ for luminance and best $(72 \%)$ for orientation. Scanpath and fixation features, especially scanpath-length, scan-strategy, fixation-duration and fixation-count were most discriminative. Individual saccade-based features were overall least discriminative.

Thus, user-identification is enhanced by some exogenous factors while suppressed by others. In order to improve performance of user-identification systems one needs to identify the disruptive exogenous factors and train the system on scanpath-/fixations-features rather than saccade-features. 
Posters

\title{
Applying the eye tracking methodology in healthcare communication research
}

\author{
Yuefang Zhou, Gerry Humphris \\ University of St Andrews, United Kingdom \\ yz10@st-andrews.ac.uk
}

Background: The eye tracking methodology has been previously applied in health research, however, little is known about how it can be adopted in studying healthcare communication.

Aim: To explore whether eye tracking methodology can be applied in healthcare communication research to study how doctors address patients' emotional expressions in clinical consultations. Research questions: (i) Do doctors look at the patients' when responding to their emotional concerns? (ii) Do patients feel more satisfied when doctors look at them while providing empathic verbal responses to their emotional concerns, compared to doctors looking elsewhere?

Methods: Three consultations between three medical demonstrators (wearing SMI glasses) and one simulated patients were video recorded; and coded (on The Observer XT) for doctors' verbal responses to patients' emotional concerns, and doctors' non-verbal eye gaze directions when the verbal responses occurred.

Results: $80 \%$ of the doctors' empathic verbal responses were delivered while looking at patients; $76 \%$ of ignoring statements were observed with fluctuating eye movements. No differences were found with patient satisfaction.

Discussion: Limited sample size prevented from significant findings; however, these preliminary data suggest that the eye tracking methodology can be a useful method to study empathy in doctor-patient communication. Gender and culture factors should be considered.

\section{Patterns of L1-L3 and L2-L3 cognate facilitation in multilingual speakers during sentence reading Agnieszka Lijewska \\ Adam Mickiewicz University in Poznan, Poland alijewska@wa.amu.edu.pl}

Cognate facilitation effect is consistently observed in bilingual experiments with isolated words and in sentence reading (esp. in semantically non-constraining context). This is interpreted as evidence for language non-selective lexical access (e.g. Van Assche et al. 2011; Duyck et al. 2007). In contrast, patterns of cognate facilitation observed with multilinguals are far less clear. Especially interesting is the facilitation produced by L2-L3 cognates which seems to be task-dependent (cf. Lemhoefer, Dijkstra \& Michel 2004; Lijewska \& Chmiel 2014; Szubko-Sitarek 2014). The present study is the first one to look into cognate facilitation effect in multilinguals during sentence reading (design adapted from Libben \& Titone 2009). Here, Polish speakers of L2 German and L3 English read English sentences while their eye movements were monitored. L2-L3 cognates, L1-L3 ones and matched controls were embedded in semantically non-constraining sentences. Preliminary analyses indicate that early eye-movement measures (e.g. first fixation duration, gaze duration and skipping), as well as late measures (e.g. go-past time, total reading time) yielded cognate facilitation for both types of cognates, but the facilitation for L2-L3 cognates was weaker than for L1-L3 ones. The results will be discussed in the broader context of models of language processing in bilinguals and multilinguals. 


\section{Some features of perception of media creolized texts}

\section{Elina Bulatova, Olga Lomtatidze}

Ural federal university, Russian Federation

elina2824@yandex.ru

Creolized media text is polycode text, which consists of signs of different semiotic systems: verbal, visual, auditory, transmitted through the media.

In order to identify features of creolized media texts perception (including the definition of the most significant for the sender and the recipient's message elements - text key concepts) step by step analysis of the magazine publications was carried out. We used following methods during our research:

1) stylistic and cognitive text analysis techniques : the constructing the text semantic structure, identifying the leading text concepts of publication;

2) experimental techniques: direct questioning, psychodiagnostic laboratory analysis of the perception of the text using the system for the registration of eye movements Eye-tracer RED 500.

The research involved 19 - 20 years old students: expectant journalists (14 people) and psychologists (16 people).

Dominant text concept, main nuclear concepts and secondary nuclear concept have been allocated based on the analysis of the text semantic structure. The experimental methods also allowed to discover the high attention of recipients to these media text elements.

In addition after statistical treatment, significant differences were found during the perception of the individual areas creolized text (significance of differences were determined using the Mann - Whitney test). 


\title{
Looking at text and pictures
}

\section{Does integration of text and picture information really aid reading comprehension? Evidence from eye-movement patterns of six graders}

\author{
Sheng Yao Tai, Yu Min Ku \\ Graduate Institute of Learning and Instruction, National Central University, Jhongli, Taiwan \\ t.ericking@gmail.com
}

Can illustration aid learning of text material? From reviewing published studies on the effects of text illustration, the answer is clear that illustrations can facilitate readers' text comprehension. Then when do text illustrations improve students' comprehension and whether illustrations have different impacts on students with high and low reading abilities are issues that need further investigation. This study aimed at addressing the aforementioned issues by using an eye-tracker to monitor 30 sixth graders' on-line processing of text and illustration while reading an illustrated science text. The reading material was an expository science text developed with the assistance from several experienced teachers to ensure that the material is age and grade appropriate.

The data of eye movement analysis show that although poor readers spent more time in reading texts and illustrations, their reading performance is poorer compared to their counterparts. Particularly interesting are the eye movement indices (second-pass-duration, times of saccade between texts and illustrations) that indicate good readers spend much more time in the integration processing than poor readers. Our results suggest that students' level of reading abilities greatly influence how they integrate text and pictorial information efficiently and appropriately.

\section{Integration of linguistic and visual information in line graph analysis: an experimental study with undergraduate students}

\author{
Luane Fragoso, Erica do Santos Rodrigues, Renê Forster \\ PUC-Rio, Brazil \\ luanefragoso@hotmail.com
}

This study aims at investigating the integration of linguistic and visual information during line graphs analysis. We assume that (i) the final output of visual processing is an abstract conceptual representation similar to the one resultant of linguistic processing (Pylyshyn, 1973,1981 ) and (ii) both types of representations can be described in terms of argumentpredicate relation (Acartürk, 2009; Acartürk \& Habel, 2012). An eyetracking experiment was conducted in which 29 Engineering undergraduate Brazilian students had to judge the compatibility of sentences and graphs. The independent variables were the congruency between the semantic content of the sentential verb (e.g. decreases) and the visual orientation of the line used in the graph (an ascendant or descendent one) and the accuracy of the information provided by the graph in terms of what is expressed in the sentence. The scanpath analysis showed that graph observation started after the reading of the entire sentence; there was a main effect of accuracy, more and longer fixations on the sentence area in the incorrect conditions and it was observed more eye-movement alternation between the two areas in the condition in which both the line and the $X$ axis presented information in a non-expected way. 


\title{
Aversive gaze during multimedia learning: emotionally charged stimuli and the personalisation effect
}

\author{
Sven Bertel, Stefanie Wetzel, Maria Reichelt, Steffi Zander \\ Bauhaus-Universität Weimar, Germany \\ sven.bertel@uni-weimar.de
}

The personalisation effect in multimedia learning states that using a personalised language style is more beneficial for learning than using a formal language style. We conducted an eye tracking study to examine whether the personalisation effect exists for learning negatively emotionally charged content. We hypothesised that in this case, opposite to the general personalisation effect, content in personalised language results in aversive gaze behaviour compared to formal language. Content consisted of text on the nature and causes of brain haemorrhages and of pictures of brain and haemorrhages. Text was presented either in personalised or formal language (e.g., "... it bleeds into your brain" vs. "... it bleeds into the brain"). Pictures were not changed between conditions. Participants' eye movements were recorded during learning. For personalised language, and according to our hypothesis, we found gaze behaviour indicating avoidance of deeper mental engagement, with reduced transitions between text and pictures. This is indicative of less mental integration of information from both representational modalities. Moreover, overall less time was spent in fixations on pictures, along with lower proportions of overall fixations, again suggesting aversive gaze. We will present conclusions for the design of instructional material for emotionally charged content.

\section{A preliminary study of reading narrative text with illustrations in EFL readers in Taiwan}

\author{
Grace M. Y. Hsieh, Sunny S. J. Lin \\ National Chiao Tung Universtiy, Taiwan, Republic of China \\ grace.myhsieh@gmail.com
}

This study aims to explore whether illustrations benefit EFL readers to read English narrative text. The narrative text and illustrations were both partitioned into three portions: exposition, climax, and resolution. Thirty-six junior high readers were randomly divided into two groups; experimental group reading narrative text on the left with the illustrations (drawing with black line) on the right while control group reading the same material on the left but without any illustration. After reading, participants were asked to answer comprehension questions. The results indicated eye movement data had no significant difference between two groups. The experimental group did not outperform the control group when reading narrative text with illustrations; run counts and fixation duration also showed no difference. However, offline data showed that the experimental group derived the significant result when answering some questions related to illustrations in posttest $(p<.05)$. It suggests that with the help of illustrations, EFL participants have better understanding of comprehension questions referring to the text with illustration but there is no significant difference on the questions referring to the text. We suggest that EFL readers are less skillful and they were not aware of using illustrations to help comprehension. 
Posters

\section{Effects of textual and audio information on paintings to object perception, attention and gaze-pattern during the observation of paintings. \\ Janos Kaldos \\ Pazmany Peter Catholic University, Hungary \\ janos.kaldos@noesis.hu}

The presentation is based on a quantitative approach to study the structure of visual attention and gaze patterns during the observation of work of arts. The main question in this research is how the context - especially the textual or audio information about the paintings - effects the structure of attention, and the perception of the paintings.

To examine this question we used quantitative eye-tracking method combined with classical survey about both figurative and abstract paintings with different textual or audio descriptions, or without any information. To measure the effects of the information we selected less known paintings, and we conducted the survey among people without any special interest or education toward art criticism.

The results of the research can be interpreted in a wider theoretical framework of the topdown and bottom-up processes of visual behaviour as the textual or audio information drive the attention from the visually „evident" objects toward the less „visible" parts of the paintings

On the other hand we can measure how the information change the overall awareness to the painting as they condition the observers to find "new" interpretable objects what are not mentioned during the audio or textual stimuli.

\section{How narrative text with illustrations affect reading performance on good and poor EFL junior high readers in Taiwan \\ Grace M. Y. Hsieh, Sunny S. J. Lin \\ National Chiao Tung Universtiy, Taiwan, Republic of China grace.myhsieh@gmail.com}

This study intends to explore how high and low ability EFL junior high students differ in reading performance on narrative text. Twenty-one participants read three trials of narrative text with illustrations. Illustrations drawn based on the narrative text. Each text and illustration was equally left-right parallel arranged in every trial. After reading, participants were asked to answer comprehension questions as posttest and their score was divided into high $(n=12)$ and low $(n=9)$ ability groups respectively. The preliminary findings were the high-ability group had longer forward saccade while low-ability group shorter. For each trial, high-ability participants had more fixations on the illustrations where richer information displayed. Low-ability participants seldom shifted their attention from text to illustrations. As for the run counts and fixation duration, no significant difference was found. Furthermore, fixation map indicated that most participants focused their attention more on the content words rather than functional words in the narrative text. Besides, offline data on the posttest with questions related to text had marginal significant result $(\mathrm{p}=.054)$ pointing out that high ability EFL readers slightly performed better than the low ability ones in text-related questions while they did not make any difference when answering the illustration-related questions. 


\title{
Reading
}

\section{Looking at a far away mountain in Kendo: eye, head, and body coordination under natural condition

\author{
Takaaki Kato \\ tiger@sfc.keio.ac.jp
} \\ Keio University, Japan}

In Kendo (Japanese fencing), "Enzan no Metsuke" is one of the most important "Waza (technique)" and is looking at the opponent with a gaze toward the mountains in the distance, taking in not only his or her face, but whole body. The purpose of this study was to analyze visual search activities of expert Kendo performers through sparring practices; threedimensional body movements of their attacking and defending behavior synchronously. The results indicated experts set their "visual pivot" on the opponent's eyes quietly most of the times while facing the opponent, even when the opponent tried to attack with the sword. It seemed to take the information not from the opponent's eyes but from their whole body by utilized properties of ambient vision system and they react instantly by using "Enzan no Metsuke". It was also found from the results of kinematic motion analysis that they controlled lower body to step toward and away from the opponent and attack or defense without moving their eyes and head influenced by the movements of the opponent. It is concluded that the concept of "Enzan no Metsuke" is related to coordination of ambient vision and motor control system.

\section{Do letter migration effects occur between two parafoveal words during reading?}

\author{
Michael G. Cutter, Denis Drieghe, Simon P. Liversedge \\ The University of Southampton, United Kingdom \\ M.G.Cutter@soton.ac.uk
}

There is a great deal of debate regarding whether words are processed in serial or parallel during reading. We investigated this by examining whether letter migration effects occur between two parafoveal words during natural reading. Letter migration effects refer to a phenomenon observed in isolated word recognition whereby having participants name one of two words (e.g. line and love) presented concurrently for a limited duration leads to participants sometimes reporting an illusory word (e.g. lone) due to crosstalk between the two presented words. During reading, we used the boundary paradigm (Rayner, 1975) to present an identity preview of words $n+1$ and $n+2$ (e.g. sip tea), a preview in which two letters had migrated between these words (e.g.tip sea), or a preview in which the same two letters had been substituted (e.g. zip pea). We hypothesised that if crosstalk occurred between words $n+1$ and $n+2$ then we may observe a preview benefit for the letter migration preview relative to the substitution preview. However, while we observed identity preview benefits there were no differences between the two incorrect preview conditions. These data provide little evidence for word $n+1$ and $n+2$ being processed in parallel. 
Posters

\title{
False positives in standard analyses of eye movements in reading

\author{
Bernhard Angele ${ }^{1}$, Titus von der Malsburg ${ }^{2}$ \\ ${ }^{1}$ Bournemouth University, United Kingdom \\ 2 UC San Diego, United States of America \\ bangele@bournemouth.co.uk
}

Standard analyses of eye movements in reading test a set of canonical dependent measures calculated for multiple regions. Although the resulting multiple comparisons increase the rate of false positive results, it is accepted standard practice not to correct for that. We investigated false positives rates through computer simulations and tested how much statistical power has to be sacrificed to control them. In 100.000 iterations, we generated six realistic data sets of eye movements for a hypothetical experiment with typical parameters. The true effect sizes of the manipulation ranged from $0 \mathrm{~ms}$ to $40 \mathrm{~ms}$. Four standard measures were analyzed using linear mixed models: first fixation duration, gaze duration, go-past time, and total viewing time. In the data sets with no true effect, the rate of false positives was $12.1 \%$, i.e. much higher than the conventionally accepted $5 \%$. A Bonferroni correction reduced false positives to $3.2 \%$ and was therefore slightly more conservative than required. The reduction in power due to the Bonferroni correction was moderate, e.g., from $90 \%$ to $75 \%$ for effect sizes of $5 \mathrm{~ms}$. Thus, contrary to conventional wisdom, the Bonferroni correction seems to be an appropriate tool for controlling false positives in reading experiments.

\section{Effects of irrelevant speech on Chinese sentence reading: An eye-tracking study}

\author{
Guoli Yan, Zhu Meng, Nina Liu, Liyuan He \\ Tianjin Normal University, People's Republic of China \\ psyyg $\mid @ 163 . c o m$
}

Many studies have shown that irrelevant speech could impair short-term memory tasks, which is known as irrelevant speech effect. However, our knowledge of more complex cognitive tasks (e.g. reading) is rather limited and even conflicting.

In present study, an eye tracker was used to investigate the influence of irrelevant speech on lexical processing during the reading of Chinese sentences. Participants either read sentences while being exposed to meaningful speech or meaningless speech (with similar acoustic properties and phonetic structure to meaningful speech) or silence. A target word of high or low lexical frequency was embedded in each sentence.

The results were as follows: (1)Meaningful speech impaired normal reading, While no difference was found between the meaningless speech and silence condition. (2)Significant word frequency effect was found. But there was neither significant main effect of background speech on local measures of eye movement nor interaction between background sound and word frequency.

These findings suggested that the semanticity of irrelevant speech contributes to disruption of sentence reading comprehension beyond the acoustic properties of the speech. Furthermore, the irrelevant speech does not impair lexical processing, and the disruption occurs on the high-level (post-lexical) of sentence reading. 


\title{
The Effect of Fatigue on Eye Movements and Metaphor Compresion in Reading
}

\author{
Ilze Laicane ${ }^{1}$, Jurgis Skilters ${ }^{2}$, Inga Jurcinska ${ }^{1}$, Gunta Krumina ${ }^{1}$ \\ ${ }^{1}$ Department of Optometry and Vision Science, University of Latvia, Latvia \\ ${ }^{2}$ Department of Communication Science, Center for Cognitive Sciences and Semantics, University of \\ Latvia, Latvia \\ ilze.laicane@lu.Iv
}

A controversial question in research on processing metaphorical language is the analysis of processing time. Different models have been proposed without reaching a consensus regarding core processing properties and their temporal dynamics (Brisard et al., 2001; Gibbs, 1992; Glucksberg, 2013; Bowdle \& Gentner, 2005).

The methodological framework of the current study is based on eye movement analysis (cp. Just \& Carpenter, 1980). Our main research perspective is to explore the possible impact of the metaphorical content on the characteristic eye movements in reading. Additionally, we analyze impact and interference of fatigue on the metaphor comprehension.

Three different texts, containing (a) simple, (b) complex or (c) no metaphors were used as experimental stimuli. Eye movements were recorded using IViewX Hi-Speed system. Our results indicate that (1) average processing time is shorter in fatigued participants and (2) contrary to non-fatigued participants the shortest fixation times are observed when reading text without metaphors. Comprehension was not affected by complexity of the text or the level of fatigue.

Acknowledgement: This study is supported by ESF project No.2013/0021/1DP/1.1.1.2.0/13/APIA/VIAA/001.

\section{The relation between reading skills and eye movements in German-speaking adolescents: A function of reading mode? Magdalena Krieber ${ }^{1}$, Katrin D. Bartl-Pokorny ${ }^{1}$, Florian B. Pokorny ${ }^{1}$, Christa Einspieler ${ }^{1}$, Peter B. Marschik ${ }^{1,2}$ \\ ${ }^{1}$ Medical University of Graz, Austria \\ 2 Karolinska Institutet, Sweden \\ magdalena.krieber@medunigraz.at}

We aimed to investigate how reading skills correlate with eye movement patterns as a function of reading mode (reading silently vs. reading aloud) in German-speaking individuals. Eye movements of 22 adolescents ( 14 females; mean age $=13 ; 6$ years;months) were tracked while reading an age-appropriate text with 150 words silently and aloud. Reading skills were determined by means of a standardized test assessing speed and comprehension during silent reading (Lesegeschwindigkeits- und -verständnistest für Klassen 6-12).

Better reading skills were associated with an increased efficiency of eye guidance during silent reading, but not necessarily when reading aloud. During silent reading, reading speed correlated with various spatial and temporal eye movement parameters; reading comprehension was primarily associated with spatial parameters. When reading aloud, the association between reading skills and eye movements was attenuated: reading speed was only related to saccadic amplitudes; reading comprehension correlated with the number and amplitudes of saccades. Interestingly, gaze duration and the number of regressions did not correlate between reading modes.

Our results suggest that silent reading skills correlate with a broad range of eye movement parameters in German-speaking adolescents when reading silently. When reading aloud, reading skills are only associated with a restricted number of eye movement parameters. 
Posters

\title{
Eye guidance in Russian: difference in processing nous vs. verbs
}

\author{
Svetlana Alexeeva
}

Laboratory for Cognitive Studies, St. Petersburg State University, Russian Federation

mail@s-alexeeva.ru

Most of our knowledge about eye movements during reading is based on studies investigating Latin-alphabet languages like English. However, Russian differs from English in a number of features which might impact on the gaze patterns when reading Russian.

The present study reports the basic eye-movement measures of natural reading in Russian based on the data recorded from 24 persons, each reading 42 sentences. The Cyrillic script, the highly inflexional morphology, and the transparent orthography in Russian results in similar patterns of fixation durations as reported for English. However, the skipping of function words and refixation rate seem to be increased for Russian readers.

We conducted supplementary analysis using linear-mixed modeling on the subset of 3557 words being either nouns or verbs. Both nouns and verbs are content words, but verbs are the nucleus of the sentence that bind all other elements in the syntactic structure. This fact might lead to reading cost for verbs during sentence reading. Our results confirm this hypothesis: readers spent less time viewing nouns than verbs even when other word-specific variables are controlled. We discuss the cognitive cost of verbs in terms of ongoing syntactic integration processes.

Funded by RGNF, N14-04-00586

\section{Children's Cognitive Processes and Learning Strategies for Reading Illustrated Biology Texts: Eye Movement Measurements Yu-Cin Jian \\ National Central University, Taiwan jianyucin@gmail.com}

Previous research suggests that multiple representations can improve science reading comprehension. This facilitation effect is premised on the observation that readers can efficiently integrate information in text and diagram formats; however, this effect in young readers is still contested. Using eye-tracking technology and sequential analysis, this study investigated children's reading strategies and comprehension of illustrated biology texts by comparing their performance to that of adults. Fourth-graders with high reading abilities and university students read a biology article from an elementary school science textbook containing two illustrations, one representational and one decorative. After the reading task, participants answered questions on recognition, textual, and illustration items. Unsurprisingly, the university students outperformed the children on all tests; however, more interestingly, eye-movement patterns differed across the two groups. The adult readers demonstrated bidirectional reading pathways for both text and illustrations, whereas children's eye fixations only went back and forth within paragraphs in the text and between the illustrations. The children also made fewer references to both text and illustration and spent less time viewing illustrations. This suggests that regardless of their high-reading ability, fourth-grade children's visual literacy is not mature enough to perceive connections between corresponding features of different representations crucial to reading comprehension. 


\title{
The role of morpho-semantic information in processing Russian verbal aspect: Evidence from pupil dilation, fixation durations and reaction times
}

\author{
Aki-Juhani Kyröläinen ${ }^{1}$, Vincent Porretta ${ }^{2}$, Juhani Järvikivi ${ }^{2}$ \\ 1 University of Turku, Finland \\ 2 University of Alberta, Canada \\ akkyro@utu.fi
}

Despite growing interest in grammatical aspect, little is known about how morpho-semantic aspectual information affects the processing of verbs. We investigated 80 Russian verbs with grammaticalized (podumat' 'think') versus lexicalized (nadumat' 'make up one's mind') perfectivizing prefixes using lexical decision with eye-tracking. We used generalized additive mixed-effects modeling to compare pupillary responses, fixation durations and reaction time data from 26 participants, aged 18-39 $(\mathrm{M}=28.8)$, to inspect the extent to which processing is impacted by distributional information of the morphological structure, aspectual type and age.

The results provide converging and complementary evidence indicating that the processing of Russian aspect is driven by an item's goodness-of-fit to the morphological construction and the relationship between root and verb frequencies. Furthermore, aspectual type and prefix family size influenced total fixation duration and modulated pupil dilation after the average reaction time. Additionally, in contrast to first fixation duration, early pupil dilation captures the frequency relationship between the root and the verb. While age did not affect reaction times or fixation durations, it influenced pupil dilation throughout the time course. This, along with capturing early effects not seen using fixation-related or behavioral measures, suggests that pupillometry uniquely contributes to understanding morphological processing.

\section{Spontaneous re-reading within sentences: The role of previously read words and the importance of word $n-1$}

\author{
Laura M. T. Lantz, Sarah J. White, Kevin B. Paterson \\ University of Leicester, United Kingdom \\ Imtl1@le.ac.uk
}

Research on eye movement control during reading has largely focused on the forward movement of the eyes. But readers frequently make regressions to re-inspect text, although little is known about eye movement control during re-reading following spontaneous regressions. Two experiments are presented that examine the processes underlying spontaneous re-reading by manipulating the availability of previously read text. All previously read words were masked, or all words beyond the previous word (word n-1) were masked. In Experiment 1, sentences were simple. In Experiment 2, sentences contained a temporary structural ambiguity, or were unambiguous. Importantly, there was more re-reading when previously read text was not masked, particularly for the ambiguous sentences in Experiment 2. The presence of meaningful text therefore influences re-reading eye movements, especially when sentences are ambiguous and so comprehension is harder. In line with very recent findings (Schotter, Tran, \& Rayner, 2014) comprehension accuracy was lower than normal when all previously read words were masked. But comprehension accuracy was similar to normal when word n-1 remained available during first-pass reading, indicating that the availability of word n-1, rather than words beyond $n-1$, is of particular importance for sentence comprehension. Implications for models of eye movement control will be discussed. 
Posters

\title{
The role of semantic transparency on the processing of English compound words: insights from individual differences
}

\author{
Daniel Schmidtke ${ }^{1}$, Julie A. Van Dyke ${ }^{2}$, Victor Kuperman ${ }^{1}$ \\ 1 McMaster University, Canada \\ 2 Haskins Laboratories \\ schmiddf@mcmaster.ca
}

Compound words vary in how transparently they represent meanings of morphemes (e.g., hogwash versus carwash). Previous eye-tracking studies on compound word recognition have borne mixed results regarding transparency (Juhasz, 2007; Frisson et al., 2008; Pollatsek et al., 2005). However, little is known about the influence of individual differences on the processing of semantic transparency during reading.

In the current study we investigated the effect of semantic transparency on 138 participants who were tested on a battery of 31 different verbal and general cognitive skills. We recorded eye-movements to 505 English noun-noun compounds. Meaning similarity between morphemes and whole words was quantified using the Latent Semantic Analysis technique (Landauer \& Dumais, 1997).

Results indicate that transparency of compounds systematically inflated first-pass reading times for participants with low scores on a range of individual differences tests. Participants performing highly on the same tests were facilitated by transparency. We hypothesise that semantic priming of compound recognition stemming from morphemes boosts processing speed for all participants. However, this advantage to transparent compounds is offset for less proficient readers who have difficulties discriminating between the multiple semantically related meanings that are activated when reading transparent compounds. Implications for theories of morphological processing are discussed. 


\title{
Saccades
}

\section{Expectation and saccade latency: Prior probability and number of alternatives}

\author{
Andreas Jarvstad ${ }^{1}$, Casimir J.H. Ludwig ${ }^{1}$, Rafal Bogacz ${ }^{2}$, lain D. Gilchrist ${ }^{1}$ \\ 1 University of Bristol, United Kingdom \\ 2 University of Oxford, United Kingdom \\ a.jarvstad@bristol.ac.uk
}

We are highly sensitive to the statistics of the world; a visitor from England stepping onto a busy Vienna street will quickly learn to look right first. Saccade latency is also affected: more probable events lead to shorter latencies. However, previous reports suggest that Hick's law - increased latency with increased number of decision alternatives (which decreases probability) - does not seem to apply to saccades. In our task, abstract symbols marked possible target locations. The shape of each symbol signalled the target probability at that location. Targets occurred at one location with $\mathrm{p}=.85$ and at the other location(s) with $\mathrm{p}=.15$ (for two alternatives) or $\mathrm{p}=.05$ (for four alternatives). The symbols were filled with luminance noise, with the target having a higher contrast than non-targets. As expected, for both two and four alternatives more probable locations resulted in shorter-latency saccades. Importantly, participants generated shorter-latency saccades to the more probable location for two than for four alternatives (group-level $\mathrm{p}=.025$ ), showing a Hick's-like slowing for identical probabilities. This shows that the number of decision alternatives and prior probability exert independent effects on saccade latency. We relate these and previous discrepant findings to adjustments in the baseline activity in evidence accumulation models.

\section{Saccade curvature as a function of movement preparation time in simultaneous and sequential dual-task paradigms

\author{
Tobias Moehler, Katja Fiehler \\ Tobias.Moehler@psychol.uni-giessen.de
} \\ Justus-Liebig-University Giessen, Germany}

Saccade curvature is a sensitive measure of spatial attention with saccades curving away from covertly attended locations. The current study investigated whether curvature away remains unaffected by movement preparation time when a perceptual task is performed concurrently, and declines with increasing movement preparation time when the perceptual task is performed before the saccade task. Participants underwent a dual-task including a visual discrimination task at a cued location and a saccade task to the same (congruent) or a different (incongruent) location. We varied saccade preparation time (time between saccade cue and Go-signal) and the occurrence of the discrimination task (immediately after the Go-signal $=$ simultaneous vs. before saccade preparation $=$ sequential). Results show that saccades curved away in incongruent relative to congruent trials. This effect remained stable in the simultaneous condition while it decreased with increasing movement preparation time in the sequential condition. Our finding indicates that a competing saccade plan is inhibited after the Go-signal during simultaneous task performance while inhibition is applied earlier and decays gradually with movement preparation time in the sequential condition. This can be explained by different time courses of attentional deployment at the discrimination target location for simultaneous and sequential dual-tasks.

Acknowledgement: DFG IRTG 1901 
Posters

\title{
Adaptive shortening and lengthening of saccade amplitude in 10-41 months-old children
}

\author{
Christelle Lemoine-Lardennois ${ }^{1,3}$, Nadia Alahyane ${ }^{1,3}$, Coline Tailhefer ${ }^{1,3}$, Thérèse \\ Collins $^{1,2,4}$, Jacqueline Fagard ${ }^{1,2,4}$, Karine Doré-Mazars ${ }^{1,3}$ \\ 1 Université Paris Descartes, France \\ 2 CNRS \\ ${ }^{3}$ Laboratoire Vision Action Cognition \\ ${ }^{4}$ Laboratoire Psychologie de la Perception \\ christelle.lemoine@parisdescartes.fr
}

\begin{abstract}
Saccadic adaptation is fundamental to maintaining the accuracy of saccadic eye movements throughout life. Saccadic adaptation can be experimentally induced in adults by displacing the target during the saccade. Adaptive lengthening and adaptive shortening of saccade amplitude are believed to rely on separate neural mechanisms. When these adaptation mechanisms emerge during development is unknown. It has been shown however that decreasing amplitude adaptation is adult-like in children aged above 8 years-old. In younger children, saccades tend to be hypometric compared to adults as if adaptation mechanisms were not fully functional. Here, we tested this hypothesis by examining saccadic adaptation in typically-developing toddlers between 10 and 41 months-old. An animated target was presented at different successive locations $10^{\circ}$ apart on a screen. While the child was looking toward the target, the target stepped in the direction opposite to the saccade over 120 trials to induce an adaptive decrease in saccade amplitude (Experiment 1) or in the same direction as the ongoing saccade over 140 trials to induce an adaptive lengthening of saccade amplitude (Experiment 2). Overall, our results show that adaptation mechanisms are in place early during development, but efficiency may differ across children.
\end{abstract}

\section{Exploring Attentional Mechanisms in Express Saccade Makers Felicity Denise Angela Wolohan ${ }^{1}$, Paul C. Knox ${ }^{2}$ \\ 1 Edge Hill University, United Kingdom \\ 2 University of Liverpool, United Kingdom wolohanf@edgehill.ac.uk}

Express saccade makers (ESMs) produce high proportions ( $>30 \%$ ) of low latency (80-130ms) express saccades (ES) in prosaccade overlap tasks. ESMs are much more frequent in Chinese compared to other populations (Amatya et al. 2012; Knox \& Wolohan, 2013), allowing us to explore them in detail. ESM performance is stable over time (Knox \& Wolohan, in press) and their antisaccade performance is poor compared to non-ESMs (Knox et al. 2012), although this is not due to a deficit in oculomotor inhibitory control (Wolohan \& Knox, 2014). We examined the performance of 18 ESMs and 46 nonESMs, classified by their performance on 200 prosaccade overlap trials, in a classic manual-response cueing paradigm. Participants completed 300 trials in which an eccentric target appearance (left/right randomised) was preceded by a spatially nonpredictive peripheral cue (valid, invalid or neutral; cue SOA $100-150 \mathrm{~ms}$ ). While expected cueing effects were observed (valid $<$ invalid and valid $<$ neutral: $\mathrm{p}^{\prime} \mathrm{s}<0.05$; neutral $<$ invalid: $\mathrm{p}<0.05$ ), there was no group difference (ESM vs nonESM; $\mathrm{p}=0.12$ ). This suggests that the oculomotor behaviour of ESMs cannot be explained by differences in attentional orienting and/or disengagement. 


\section{Saccadic preparation vs. covert attention: event related potentials to stimulation at a cued location \\ Rinat Hilo ${ }^{1}$, Shlomit Yuval-Greenberg ${ }^{1,2}$ \\ ${ }^{1}$ School of Psychological Sciences, Tel Aviv University, Israel \\ 2 Sagol School of Neuroscience, Tel Aviv University, Israel \\ missmuffett2010@gmail.com}

The premotor theory of attention posits that spatial attention derives from a weaker activation of the same cortical mechanism that underlies oculomotor preparation. Therefore, according to this theory, covert attention and saccade preparation are only quantitatively but not qualitatively different.

We tested this hypothesis using simultaneous EEG and eye-tracking in subjects while they performed a variation of the Posner procedure. Each trial started with a cue pointing either left or right and ended with a target, which was either valid $(70 \%)$ or invalid $(30 \%)$ with the cue. In $80 \%$ of the trials a checkerboard probe was presented bilaterally at varying latencies between the cue and the probe. In separate blocks, subjects were instructed to respond to target appearance by either: performing a saccade toward it (saccade-preparation) or by pressing a button indicating its location (covert attention). In both cases subjects were instructed to ignore the probe. We analyzed ERP relative to probe onset.

Findings with 6 subjects revealed enhanced P1 component to saccade preparation vs. covert attention. This suggested a quantitative difference between the two tasks. However, the scalp topography of this effect revealed a non-linear enhancement. That is, in contrast to the tested hypothesis, the two processes differed qualitatively.

\section{Saccadic Eye Movement Abnormalities in Children with Epilepsy} Judith Fiona Lunn ${ }^{1}$, Tim Donovan ${ }^{2}$, Damien Litchfield ${ }^{3}$, Trevor Crawford ${ }^{1}$, Robert Davies ${ }^{1}$

${ }^{1}$ Lancaster University, United Kingdom

2 Cumbria University, United Kingdom

${ }^{3}$ Edge Hill University, United Kingdom j.lunn1@lancaster.ac.uk

Childhood-onset epilepsies are a heterogeneous group of neurological disorders that are associated with an increased risk of cognitive deficits and behavioural problems. This includes children with 'epilepsy only' (i.e. average global intelligence and normal neurological examinations). Impaired executive function and attention problems are reported frequently and difficulties can persist beyond remission. The relationship between epilepsy-related factors (medications, age at onset, duration), neurocognitive development and behavioural outcomes remains unclear. Saccade tasks have been used to assess the functional integration of sensorimotor and cognitive processes across different neurodevelopment disorders. This study assessed prosaccade and antisaccade task performance in 27 children with 'epilepsy only' (11 unmedicated) and 48 healthy control children aged 8-18 years. Measures included latency, gain, peak velocity, and antisaccade error rate. Results showed medicated patients had significantly increased antisaccade peak velocity, gain and error rate compared to controls. Age-related changes in latency observed in controls were absent in patients, whereas epilepsy-developmental factors better accounted for latency and peak velocity in the patient group. Furthermore, in patients, increased latency, velocity and error rate significantly predicted greater attention problems. Childhood epilepsy is associated with atypical development of oculomotor skills that are important potential markers for poor behavioural outcomes. 
Posters

\title{
On saccade programming as a function of stimulus complexity: Estimating the population-averaging window for simple shapes, textured discs and natural objects
}

\author{
Lotje van der Linden ${ }^{1}$, Gregory Zelinsky ${ }^{2}$, Françoise Vitu ${ }^{1}$ \\ ${ }^{1}$ Aix-Marseille University, CNRS, LPC UMR 7290, Marseille, France \\ 2 Department of Psychology, Stony Brook University, New York, United States \\ I.vanderlinden@cogsci.nl
}

In visual displays containing two stimuli, saccades reveal a global effect (GE): Even though participants aim for one of the stimuli, their eyes land in between the two. The GE presumably results from population averaging in the superior colliculus (SC). Neurons in the retinotopic sensory/motor maps have overlapping receptive/movement fields. Therefore, overlap in the recruited populations causes activity to peak at the intermediate location. The GE diminishes when the distance between the two recruited populations exceeds a threshold. Due to nonhomogeneous afferent mapping to the SC, this averaging window is invariant across the SC. Yet, the corresponding distance in visual space increases with eccentricity (Casteau and Vitu, ECEM, 2009). To investigate whether this mechanism generalizes to saccades towards more natural stimuli, we estimated the averaging window for target-distractor (TD) pairs of increasing complexity: simple shapes, objects, and, as an intermediate level, discs having texture matching the objects. We varied distance and eccentricity of the TD arrangement along various axes. We found that for shapes and discs, the critical distance for a GE increased with eccentricity in visual space, but was constant in collicular space. For objects, the pattern was similar, although variance in landing positions was larger.

\section{The spatio-temporal modulation of saccades in a double-step paradigm}

\section{Soazig Casteau, Robin Walker}

Department of Psychology, Royal Holloway University of London, Egham, Surrey, United Kingdom soazig.casteau@rhul.ac.uk

Studies of saccades made in the double-step paradigm have shown that saccade landing position is modulated by the time between target-step and saccade initiation. At short delays, first saccade land accurately on the second location, for long delays first saccades are directed towards the first location; whereas at intermediate delays saccades land at an intermediate position (i.e. saccade averaging). This spatial averaging effect has been interpreted as the integration, during a "time-window", of the spatial representations of the two target locations (Becker \& Jürgens, 1979).

Here the "time-window" hypothesis was investigated in relation to the assumption that saccade averaging reflects the distributed spatial coding in the purported collicular saliency map. We hypothesized that for intermediate delays, landing position would be modulated depending on target eccentricity and step-size.

Target direction, spatial separation between target-steps and inter-stimulus interval (ISI) were manipulated. As expected, for intermediate ISI's saccade averaging was observed, but only for short spatial separations; for large separations saccades landed accurately on the target. These effects on landing position indicate that the computation of saccade amplitude depends not only on the "time-window" but also on the spatial separation, suggesting that the spatial integration fields coding for target positions are modulated over-time. 


\author{
Saccade plan cancellation and overlap during free viewing \\ Esther Xiu Wen $\mathrm{Wu}^{1,2}$, Fook-Kee Chua ${ }^{2}$, Shih-Cheng Yen ${ }^{1,3}$ \\ ${ }^{1}$ Singapore Institute for Neurotechnology (SINAPSE), National University of Singapore \\ 2 Department of Psychology \\ , National University of Singapore \\ ${ }^{3}$ Department of Electrical and Computer Engineering, National University of Singapore \\ estherwu@nus.edu.sg
}

Previous studies have shown that (a) saccade plans could be cancelled before they were completed, and (b) saccade plans could overlap, such that a saccade plan to an object could start even while the plan to another object was in progress. In studies using the double-step task for example, saccade targets were restricted to a few, experimenter-defined locations. In this study, we examined whether saccade plan cancellation and overlap mechanisms could be extended to the free-viewing of real objects in images. For each trial, we constructed sets of two images, each containing five objects at unique locations. Image 1 was presented for several fixations, before Image 2 was presented, presumably while saccade planning to an object in Image 1 was ongoing. The crucial finding was that the first saccade following the transition was sometimes executed towards objects in Image 2, and not an object in Image 1 , suggesting that the earlier saccade plan to an Image 1 object had been cancelled. The temporal data also suggested that plan of the first post-transition saccade started before an earlier saccade plan to an Image 1 object was executed. Our results support saccade plan cancellation and saccade plan overlap during free viewing.

\title{
Retinotopic coding of value for saccadic eye movements \\ Alexander C. Schütz, Julia Zimmermann \\ Justus-Liebig Universität Gießen, Germany \\ Alexander.C.Schuetz@psychol.uni-giessen.de
}

Expected reward attracts saccades and reduces latencies. Here we investigated if these effects are encoded in a retinotopic or spatiotopic frame of reference.

Participants were instructed to saccade to targets appearing in the periphery. In baseline trials, initial fixation was at the center of the screen and targets could appear $8^{\circ}$ to the left or to the right or at both sides simultaneously. One side yielded a high reward and the other side a low reward, leading to faster responses and more choices for the highly rewarded side. In the following test trials, retinotopic and spatiotopic reference frames were disentangled since initial fixation was at $16^{\circ}$ to the left or to the right. Targets could appear at $8^{\circ}$ distance to the left or to the right of initial fixation. Participants were instructed that these targets yielded rewards too, but the exact values were undisclosed until the end of the experiment. In a questionnaire about the distribution of rewards, participants' estimations matched a retinotopic reference frame most often. Consistently, saccade latencies and saccade choices were more compatible with a retinotopic than a spatiotopic reference frame. This suggests a primacy of retinotopic coding of value for eye movements. 
Posters

\title{
The role of cortical and subcortical regions in modulating exogenous and endogenous control of gaze.
}

\author{
Michele Furlan, Andrew T. Smith, Robin Walker \\ Royal Holloway University of London, United Kingdom \\ Michele.Furlan@rhul.ac.uk
}

Here we applied a combination of univariate (GLM) and multivariate (MVPA) analysis to measure the hemodynamic activity associated with target selection (a) in cortical regions associated with the high-level control of eye movements and in the Superior Colliculus (SC). A 3T fMRI study examined the Blood Oxygen Level Dependent (BOLD) response in cortical and sub-cortical brain regions while participants prepared and executed saccades toward a target (prosaccade) or away from it (antisaccade). Antisaccades produced a larger hemodynamic response than prosaccades in the Dorsolateral Prefrontal Cortex (DLPFC), the Frontal Eye Field (FEF) and the Intraparietal Sulcus (IPS) cortical regions but not in the SC. MVPA analysis was able to distinguish between two different patterns of activity for prosaccade and antisaccade in the cortical areas, but not in the SC. A further finding was that the saccade-related BOLD response did not show the expected contralateral mapping in terms of saccade direction, in either the cortical regions or SC. This study has confirmed the high-level activity in cortical regions but not in the SC and provides evidences of a consistent lack of contralateral bias in the BOLD signal.

\section{Bottom-up or top-down? An MEG approach to disentangle oculomotor selection strategies}

\author{
Davide Paoletti ${ }^{1}$, Wieske van Zoest ${ }^{1}$, Christoph Braun ${ }^{2}$ \\ 1 University of Trento, Italy \\ 2 University of Tubingen, Germany \\ davide.live@hotmail.it
}

When in time a response is made seems critically important in defining whether visual selection is driven by physical stimulus-salience or goal-driven control. In study 1 we investigated whether observers are able to control the timing of selection and regulate the trade-off between stimulus- and goal-driven influences. Participants were instructed to make a saccade to an orientation target while a cue instructed them to do so either 'fast' or 'accurate'. Relative salience of the target and an irrelevant distractor was manipulated. Performance in the fast-cue condition appeared to be driven by stimulus salience, while in the accuratecue condition saccades were guided by the search template. A main effect of cue suggests that preparation can bias mechanisms of selection prior to saccadic execution. In study 2 we investigate the neural mechanisms underlying saccadic preparation and control. Using a similar task, MEG and eye movements were measured concurrently. Preliminary results of 6 participants revealed stronger activity in the lateral intraparietal area when target was selected as opposed to distractor. Moreover, correct saccades were predicted by lower alpha activity in the pre-stimulus period compared to erroneous responses. Further work will help to increase our understanding in the neural mechanisms underlying oculomotor control. 


\title{
Presaccadic potentials at the experimental scheme with distracters (stimulation of dominant and undominant eye)
}

\author{
Victoria Moiseeva $^{1,2}$, Maria Slavutskaya ${ }^{1}$, Valeriy V. Shulgovskiy ${ }^{1}$, Natalia Fonsova ${ }^{1,2}$ \\ ${ }^{1}$ Lomonosov Moscow State University and Higher School of Economics, Russian Federation \\ 2 Higher School of Economics, Center for Cognition and Desition Making \\ moiseeva.victoria@gmail.com
}

The goal of our research was to analyze spatial-temporal parameters of saccades and presaccadic EEG-potentials at the simultaneous presentation of the target and distracting stimuli to the dominant and undominant eye. 14 healthy right-handed volunteers participated in the study. Target and distracting peripheral visual stimuli were presented simultaneous monocularly on the monitor in various spatial combinations. The main asymmetry in saccadic LP was revealed at the presentation of target and distracting stimuli in the left visual hemyfield at the distance 5 degrees between them. The results show that early potentials N1 and P1 were higher in amplitude and dominated in medial and controlateral parietal-occipital areas. The amplitude of the potential N2 at the stimulation of the right eye increased in the case when target stimulus was at the same location than at the previous realization. It's possible that $\mathrm{N} 2$ component is connected with memory attention. N2 amplitude was higher when the distance between target and distracting stimuli was 15 degrees in comparison with the distance 5 degrees. It's corresponded with LP data. The findings show an active role of attention and decision-making in saccade programming. Part of the study was executed at the support of RFBR (the projects No 14-04-01634).

\section{The Price of Saccades}

\author{
Felix Schüler ${ }^{1,2}$, Vanessa Nunnendorf ${ }^{1}$, Constantin Rothkopf ${ }^{3}$, Peter König ${ }^{1}$ \\ 1 Universität Osnabrück, Osnabrück, Germany \\ 2 Technische Universität Berlin, Berlin, Germany \\ 3 Technische Universität Darmstadt, Darmstadt, Germany \\ fschueler@posteo.de
}

The limited capacity of human visual-working memory leads to a strategy in which subjects use many saccades and little working-memory in classical block copying tasks. Here we examine the trade-off between the number of eye-movements and visual working-memory by parametrically manipulating the cost of saccades through time delays in a gaze-contingent paradigm. To this end, we modify a block-copying experiment with simultaneous tracking of eye movements by introducing a temporal penalty for saccades towards the template region. We find that the cost of saccades has a significant effect on subjects' strategy. The number of saccades to the template-region decreased under the penalty, while subjects' use of memory increased, reflected by higher numbers of fixations and saccades to the template while in the template-region. We also find that fixation durations are modulated by the subsequent fixation-target. In all conditions, durations of fixations preceding a saccade into the template-region were significantly longer than previous fixations within that same region. The present results demonstrate that visual information seeking and memory usage are not fixed but highly susceptible to the costs incurred. However, the functional dependence can not be explained by the indirect control theory of saccades, but requires new theoretical approaches. 
Posters

\title{
Influence of stimulus exposure time in the evaluation of saccades measured with a new visual analyzer
}

\author{
Juan Carlos Ondategui Parra, Rosa Borras García, Elena Lopez García, Arnau Fado, Mikel \\ Aldaba, Jaume Pujol Ramo \\ Davalor Research Center (DRC) - Politechnical University of Catalonia, Spain \\ ondategui@oo.upc.edu
}

Purpose: To determine the influence of exposure time in saccades movements implemented in a prototype of a new fully autonomous and automated vision analyzer (Eye and Vision Analyzer, EVA, DAVALOR, Spain) that records eye movements while the patient watches a true-3D short video game.

Methods: Measurements were performed in a group of 18 young (range: 20 to 30) healthy subjects. Monocular visual acuity for near distance equals or better than $0.0 \log$ MAR was required. Clinical methods consisted of register the binocular saccades movements with an eye tracker while the stimulus were showed on a display for each eye. We measured the accuracy (mean of differences between position stimulus and eye) and stability (standard deviation) of saccades amplitude for 10 degree and exposure times of $0.5,1$ and 2 seconds. We analyze the results for right eye, left eye, dominant eye (DE) and no-dominant eye (NDE).

Results: See values on table attached

Conclusions: We did not observe differences in accuracy and stability at different exposure times stimulus studied. The DE has better accuracy and stability than the NDE when the exposure time is $1 \mathrm{~s}$ and $2 \mathrm{~s}$. In binocular conditions the dominant eye has always better accuracy and stability.

\section{Motor preparation and attentional benefits: potential targets and resolving competition

\author{
${ }^{1}$ University of Geneva, Switzerland \\ ${ }^{2}$ Laboratoire Psychologie de la Perception, Université Paris Descartes, France
} \\ Michael Puntiroli ${ }^{1}$, Dirk Kerzel ${ }^{1}$, Sabine Born ${ }^{1,2}$ michael.puntiroli@unige.ch}

Movements, prepared through complex modulation in multiple brain centers, require activation of inhibitory components believed to assure execution is not triggered prematurely (Cohen, Sherman, Zinger, Perlmutter \& Prut, 2010). It has been suggested that the preparation of an eye movement towards one of two potential targets relies greatly on such inhibitory components (Lawrence, John, Abrams \& Snyder, 2008), due to heightened competition. Our goal was to verify the attentional implications associated with preparing eye movements towards two locations, and the subsequent rejection of one of these. We employed a dual-task paradigm, primarily requiring the rapid execution of saccades to one of two potential targets, while discriminating an asymmetric cross flashed at one of four locations (two potential target locations and two controls). Our results show attentional enhancement at the potential target locations compared to the controls. In the time immediately following target appearance a dip in discrimination performance was observed at the saccade target location, while performance at the rejected location continued to increase, only to decrease at a later stage. Preparing eye movements appears to carry perceptual benefits and the process of competition resolution seems to have direct effects on the attentional benefits found at these locations over time. 


\section{A perceptual asymmetry may be determined by eye dominance Jérôme Tagu ${ }^{1}$, Karine Doré-Mazars ${ }^{1}$, Christelle Lemoine-Lardennois ${ }^{1}$, Dorine Vergilino-Perez ${ }^{1,2}$ \\ ${ }^{1}$ Laboratoire Vision Action Cognition, EA n ${ }^{\circ} 7326$, Institut de Psychologie, IUPDP, INC, Université Paris Descartes, Sorbonne Paris Cité \\ 2 Institut Universitaire de France jerome.tagu@free.fr}

The dominant eye is the one used to sight in a camera. Neuroimaging studies have shown that dominant eye is preferentially linked to the ipsilateral primary visual cortex (Erdogan et al. 2002; Shima et al., 2010), that deals with the controlateral visual field due to optical pathways' crossing. Recently, Vergilino-Perez et al. (2012) showed with binocular recordings of eye movements that participants could exhibit weak or strong eye dominance. The present study investigates if the link between dominant eye and ipsilateral primary visual cortex induces a better perceptual processing in the visual field controlateral to the dominant eye compared to the ipsilateral one. To verify this assumption, participants were submitted to global and remote distractor effect situations (Walker et al., 1997). A greater effect of the distractor presented controlaterally to the dominant eye is expected on saccadic amplitude (global effect) and latency (remote distractor effect) only for participants with strong eye dominance. No differences on saccadic parameters are expected between the two visual fields in the case of weak eye dominance. Our preliminary results on 114 participants provide important information on the role of eye dominance in perception and action processes. 
Posters

\title{
Smooth pursuit
}

\author{
Adaptation of smooth pursuit in response to an intra-saccadic change \\ in target velocity \\ Giulietta S. Monasso ${ }^{1}$, Maarten A. Frens ${ }^{1,2}$, Jos N. van der Geest ${ }^{1}$ \\ ${ }^{1}$ Neuroscience, Erasmus MC Rotterdam, The Netherlands \\ 2 Erasmus University College, Rotterdam, The Netherlands \\ giuliettasophiemonasso@gmail.com
}

We investigated how human smooth pursuit eye movements adapt to an unexpected change in target velocity during the catch-up saccade. In each trial of all seven experimental conditions, participants looked at a central fixation target that started to move to the left or right with a fixed velocity. In order to pursue the target accurately, observers had to make catch-up saccade, which was followed by post-saccadic pursuit. We measured the eye velocity for $100 \mathrm{~ms}$ after the catch-up saccade.

In pre-adaptation trials, pursuit velocities nicely matched the pre-saccadic target velocity of 10 degrees/s. Subsequently, in 50 adaptation trials, we changed the target velocity at the moment of the catch-up saccade consistently to $0,25,50,75,125,150$ or 175 percent of the initial target velocity, depending on the condition.

We observed gradual decreases or increases of pursuit velocities during the adaptation trials. In all conditions, the difference in pursuit velocities between 10 post-adaptation and 10 pre-adaptation trials was significant. In these test trials, the target was extinguished at the moment of the catch-up saccade to avoid visual feedback. On average, in 29 subjects, the change in pursuit velocity was about 32 percent of the change in target velocity.

\section{Random errors in adaptation of smooth pursuit and saccades}

\author{
Berna M. Isik, Marcel de Jeu, Maarten A. Frens, Jos N. van der Geest \\ Neuroscience, Erasmus MC Rotterdam, Netherlands \\ bernamerveisik@gmail.com
}

Adaptation of smooth pursuit and saccades relies on the translation of an eye movement error into subsequent changes in eye velocity or amplitude. In classical saccade adaptation paradigms, the size of the target displacement is consistent over subsequent trials, resulting in a gradual change in saccadic amplitudes. As for smooth pursuit, changes in eye velocity can be induced by consistently changing target velocities after the catch-up saccade in a ramp paradigm. These two paradigms provide information about overall adaptation of eye movement behaviour, but do not provide insight on trial-by-trial adaptation. In a modified saccade adaptation paradigm, we randomly changed the intra-saccadic target displacement in consecutive trials. Similarly, we randomly changed target velocity following the catch-up saccade to a moving target in a modified smooth pursuit adaptation paradigm. This means that in all adaptation trails, a mismatch between eye movement and target behaviour was induced. We quantified the contributions of these eye movement errors to saccade amplitude or to smooth pursuit velocity in the present trial using state-space models. Presenting random errors does not induce overall adaptation of post-saccadic smooth pursuit velocities and saccade amplitudes. However, it seems to affect eye movement behaviour on a trial-by-trial basis. 


\title{
Linear motion processing in the brain during smooth pursuit eye movement: A spatiotemporal localization
}

\author{
Tomoko Yonemura ${ }^{1}$, Hisashi Toyoshima ${ }^{2}$, Reika Endo ${ }^{3}$, Takahiro Yamanoi ${ }^{3}$ \\ 1 Meikai University \\ 2 Japan Technical Software \\ 3 Hokkai-Gakuen University \\ yonemurat@gmail.com
}

\begin{abstract}
A linear moving white full circle on a CRT was presented to subjects. Moving patterns were downward, upward, to the right and left. A random movement of the disk was presented to subjects in comparison to other movements. These five movements were presented at random to the subjects. Subjects were requested to trace the stimulus. Meantime, electroencephalograms (EEG) were recorded. The EEG was summed in each movement and the equivalent current dipole localization (ECDL) was done to estimate the source in the brain. As results, the dipoles were localized to the MT at latency from $80 \mathrm{~ms}$ to $120 \mathrm{~ms}$, and after on the intraparietal sulcus from $140 \mathrm{~ms}$ to $180 \mathrm{~ms}$, on the precentral gyrus from $210 \mathrm{~ms}$ to $250 \mathrm{~ms}$ and on the frontal eye field from 210 to $260 \mathrm{~ms}$. The temporal tendency is the same for all subjects. The direction of estimated dipole corresponded with the opposite movements. And the dipole on the superior colliculus was estimated, this organ is supposed to correspond with the eye movement. Also a dorsal pathway and a ventral pathway were found.
\end{abstract}

\section{The influence of stimulus-predictability on directional pursuit accuracy in man and monkey}

\author{
Andre Kaminiarz, Frank Bremmer \\ Philipps-Universität Marburg, Germany \\ andre.kaminiarz@physik.uni-marburg.de
}

When humans pursue a moving visual target the direction of their eye movement deviates systematically from the target direction. Eye movement directions are shifted towards the diagonals for target directions close to cardinal directions (horizontal and vertical) [Krukowski \& Stone, Neuron, 2005]. Here, we aimed to determine whether (i) this effect can also be observed in nonhuman primates and whether (ii) it is affected by path predictability (circular pursuit).

In different experimental setups, human observers and monkeys tracked a target that moved either on a linear or circular pathway (smooth pursuit). Eye movements were sampled at $500 \mathrm{~Hz}$ using infrared eye trackers and the deviation of pursuit direction from target direction was calculated.

In humans and non-human primates (NHPs) we observed a shift of eye movement direction towards the horizontal axis for both, linear and circular pursuit. Error magnitude was similar under both conditions.

We conclude that deviations of pursuit direction from target direction are a robust phenomenon which can be observed in both humans and NHPs. Different from our hypothesis, it is not affected by cognitive factors such as path predictability.

Supported by DFG-CRC/TRR-135/A1. 
Posters

\title{
Stimulus speed influence in evaluating pursuit movements measured with a new visual analyzer

\author{
Jaume Pujol Ramo, Mikel Aldaba, Arnau Fado, Elena Lopez García, Rosa Borras García, \\ Juan Carlos Ondategui Parra \\ Davalor Research Center (DRC) - Politechnical University of Catalonia, Spain \\ pujol@oo.upc.edu
}

Purpose: To determine the stimulus speed in the assessment of pursuit movements implemented in a prototype of a new fully autonomous and automated vision analyzer (Eye and Vision Analyzer, EVA, DAVALOR, Spain) that records eye movements while the patient watches a true-3D short video game.

Methods: Measurements were performed in a group of 18 young (range: 20 to 30) healthy subjects. Monocular visual acuity for near distance equals or better than $0.0 \log$ MAR was required. Clinical methods consisted on registering the pursuit movements with an eye tracker while the stimulus were showed on a display for each eye. We measured the stimulus speed accuracy in terms of root mean square (RMS) in clockwise and counterclockwise with stimulus speed of 15, 30 and 45 degrees/second. We analyze the results for dominant eye (DE) and no-dominant eye (NDE).

Results: See values on tables 1, 2 and 3 attached

Conclusions: The slightest error is given for $30^{\circ} / \mathrm{s}$, followed by $15^{\circ} / \mathrm{s}$ and finally $45^{\circ} / \mathrm{s}$. Clockwise and counterclockwise results seem to be similar. The dominant eye makes better follow than non-dominant, both binocular and monocular fixation. The binocular tracking is only slightly better than monocular.

\section{Smooth pursuit detection using multiple Hidden Markov Models and Mahalanobis distances}

\author{
Simon Schenk, Stefan Hörmann, Gerhard Rigoll, Michael Dorr \\ Technische Universität München, Germany \\ simon.schenk@tum.de
}

We detect smooth pursuit (SP) gestures using Machine Learning methods.

Each possible state (e.g. SP in different directions, fixation, and an 'uncertain' state) is represented by a separate Hidden Markov Model (HMM), and the HMM outputs are classified using the Mahalanobis distance.

We recorded eye movements from 5 participants (4m/1f) using an EyeLink 1000 in remote configuration at $500 \mathrm{~Hz}$. The stimuli always showed both static and moving objects simultaneously; in a blocked design, participants had to either fixate the static or follow the moving target. Overall, 300 fixation episodes of 10 s each and 600 smooth pursuit episodes ranging from 2.5 to $5.7 \mathrm{deg} / \mathrm{s}$ were collected and hand-labeled.

We pre-processed the data by removing saccades and blinks, and the remaining segments were smoothened with a low-pass filter. We then used one half of the data set to train each HMM with their respective user state; the distance classifier was trained on the HMM outputs for another quarter of the data, and the remaining quarter served as test set.

Our proposed method achieves excellent offline results with a precision of 0.89 and recall of 0.96. We are currently extending our method for online classification of eye movements. 


\section{Comparison of similarity metrics for smooth pursuit eye movements Filip Děchtěrenko ${ }^{1}$, Matěj Kocián ${ }^{1}$, Jiří Lukavský ${ }^{2}$ \\ ${ }^{1}$ Faculty of Mathematics and Physics, Charles University in Prague, Czech Republic 2 Institute of Psychology, The Czech Academy of Sciences, Czech Republic filip.dechterenko@gmail.com}

In eye tracking experiments with dynamical stimuli, comparing scan patterns - as eye movements in the scene over time - is of special importance. Although several methods for comparing similarity of scan patterns exist, it is unclear how those metrics are related. Additionally, it is unclear how these methods are affected when a systematic noise is added to the data. Three metrics were used for the comparison: Normalized Scanpath Saliency, Levenshtein distance and discrete Fréchet distance.

For the simulation, we created artificial scan patterns modeled as Gaussian random walk mimicking the real eye tracking data from our previous experiments. We tested each metric, whether it can successfully match the original scan pattern to a modified scan pattern among a set of other random scan patterns. The modified versions included: vertical or horizontal translation, rotation, scaling, and flipping around $\mathrm{x}$ - or $\mathrm{y}$ - axis similarly as in Dewhurst et al. (2012).

Results show that Fréchet distance outperformed other two conventional metrics for comparing scan patterns and for some modification it achieved perfect performance. Normalized scanpath saliency showed best discrimination capabilities when scan pattern were flipped around axes.

\section{Evaluation of Smooth Pursuit in Individuals with Central Field Loss Natela Shanidze, Elena Potapchuk, Stephen Heinen, Preeti Verghese \\ Smith-Kettlewell Eye Research Institute, United States of America natela@ski.org}

Smooth pursuit eye movements have traditionally been closely tied to the fovea. Little is known, however, about pursuit in individuals with central field loss (CFL). CFL is often associated with the loss of the fovea, eccentric viewing, and loss of binocular vision, as the areas of residual vision may not correspond in the two eyes. All of these features can impede smooth pursuit. To determine whether individuals can pursue and quantify how well, we present eye movement data for 6 individuals with differing degrees of CFL. Subjects pursued a 1-degree circular target in a step ramp paradigm (Rashbass 1961), moving in 6 possible trajectories at 5, 10 and $15 \mathrm{deg} / \mathrm{s}$. Experiments were performed with binocular and monocular viewing, and eye movements of both eyes were recorded (Eyelink). To quantify pursuit, velocity gains and error, defined as deviation of eye position from the target trajectory, were used. Unlike in the control participant, gains in patients were abnormal (0.13-4.62) and varied with target trajectory. Patients exhibited more error between target and eye position than a healthy control. Binocular viewing reduced error only for patients without a large binocular scotoma overlap, but did not affect gain overall. 
Posters

\title{
Changes in visual sensitivity during smooth pursuit and saccadic eye movements
}

\author{
Doris I. Braun, Alexander C. Schütz, Karl R. Gegenfurtner \\ Justus-Liebig University Giessen, Germany \\ doris.braun@psychol.uni-giessen.de
}

Eye movements and attention adjust visual sensitivity continuously. In particular, during saccades, visual sensitivity is reduced by an active process suppressing low-frequency luminance stimuli, but not isoluminant color stimuli (Burr, Morrone \& Ross, 1994). During smooth pursuit, sensitivity is decreased for the detection of low-frequency luminance stimuli, but increased by $16 \%$ for color stimuli (Schütz, Braun, Kerzel \& Gegenfurtner, 2008). We investigated whether these sensitivity adjustments interact during saccades to moving targets.

As baseline, contrast thresholds were determined for the detection of red isoluminant lines flashed for $8 \mathrm{~ms}$ on a gray screen 2 degs above or below a central fixation point. Then detection performance for two contrast levels of the horizontal line was measured during pursuit with step-ramps and during saccades with steps to stationary and moving targets at different times relative to eye movement target onset. Contrary to our expectations observers showed a marked saccadic suppression not only for low spatial frequency luminance stimuli, but also for the detection of isoluminant and high spatial frequency stimuli. This was the case for both, saccades to static and moving targets.

Whatever sensitivity improvement there is during pursuit, it is suspended during the initial saccade to a moving target. 


\title{
Vergence
}

\section{A case-study on relations between dynamic and static vergence states: vergence velocity, heterophoria, objective and subjective fixation disparity \\ Wolfgang Jaschinski \\ Leibniz Research Centre for Working Environment and Human Factors, Germany jaschinski@ifado.de}

The velocity of vergence eye movements can be different in the convergent and divergent direction. This dynamic vergence asymmetry is known be related to static vergence measures as fixation disparity and heterophoria. Previous reports are extended by the present intraindividual approach: one individual performed a series of 14 sessions over 3 weeks with frequent repeated vergence step responses, similar as in vergence training. Disparity step stimuli subtended 1,2 and $4 \mathrm{deg}$ at a $70 \mathrm{~cm}$ viewing distance. The asymmetry between convergent and divergent vergence velocity was correlated with three static vergence measures resulting in $\mathrm{r}=0.47$ for the heterophoria (measured with a Maddox rod procedure), $\mathrm{r}=0.59$ for the subjective fixation disparity (measured with nonius lines) and $r=0.54$ for objective fixation disparity (measured with a video eye tracker). Further, objective fixation disparity was correlated with the subjective fixation disparity $(\mathrm{r}=0.47)$ and with the heterophoria $(\mathrm{r}=0.83)$. Correlations larger than 0.46 are significant $(\mathrm{p}<0.05, \mathrm{n}=14)$. Thus, the variance across repeated intra-individual observations reveals correlations between dynamic and static vergence measures. This suggests a functional relation within individuals, e.g. the state of a faster convergent (divergent) velocity appears to be associated with the state of a more eso (exo) fixation disparity or heterophoria, respectively.

\section{Vergence responses to vertical disparity during reading and lexical identification}

\author{
Mirela Nikolova ${ }^{1}$, Stephanie Jainta ${ }^{2}$, Hazel I. Blythe ${ }^{1}$, Simon P. Liversedge ${ }^{1}$ \\ 1 University of Southampton, United Kingdom \\ 2 Leibniz Research Centre for Working Environment and Human Factors (IfADo) \\ M.Nikolova@soton.ac.uk
}

Binocular disparity during reading is present in a significant proportion of fixations, but does not typically cause diplopia. The effects of both naturally occurring and stereoscopically manipulated horizontal disparities during reading have been previously explored in detail, whereas little is known about vertical fixation disparities. We investigated whether horizontal and vertical disparities, and the associated vergence responses, are functionally distinct in reading. We measured the magnitude of vertical fixation disparity and vergence adjustments during natural sentence reading (Experiment 1 ) and when disparity was stereoscopically induced in a lexical decision task (Experiment 2). The results from Experiment 1 indicated that the magnitude of vertical disparities during natural reading was considerably reduced in comparison to horizontal disparities. Additionally, when comparing binocular and monocular viewing conditions, clear differences were observed in horizontal, but not in vertical vergence responses. In Experiment 2, when varying levels of vertical disparity were stereoscopically imposed on single words, no significant vergence adjustments were made. Furthermore, we found no evidence that the vertical disparity manipulation impacted on lexical processing. These findings indicate that there is a clear dissociation between horizontal and vertical vergence responses during reading, which is likely related to their distinct functional roles. 
Posters

\title{
Monocular and binocular calibrations in evaluating fixation disparity with video-based eye-tracker
}

\author{
Aiga Svede $^{1}$, Elina Treija ${ }^{1}$, Wolfgang Jaschinski ${ }^{2}$, Gunta Krumina ${ }^{1}$ \\ ${ }^{1}$ University of Latvia, Department of Optometry and Vision Science, Riga, Latvia \\ 2 The Leibniz Research Centre for Working Environment and Human Factors (IfADo), Dortmund, \\ Germany \\ aiga.svede@lu.Iv
}

When measuring fixation disparity, the question arises as to whether monocular or binocular calibration is more precise and physiologically more appropriate? The aim of this study was to test the accuracy of monocular and binocular calibration and to evaluate the effect of the calibration procedure on the measurement of fixation disparity if video-based eye tracker is used. The results showed that the accuracy of calibration is not systematically different for pre- versus post-calibration and is not affected by the spatial characteristics of calibration targets. However, monocular calibration showed less accurate calibration results compared to binocular calibration. Excluding inaccurate calibration results, we observed that fixation disparity measurements have larger dispersion after monocular calibration. Four out of ten participants had significantly different individual fixation disparities with the two types of calibration procedures; typically, the monocularly-calibrated fixation disparity was larger than the binocularly-calibrated fixation disparity. The monocularlycalibrated fixation disparity was stronger affected by the heterophoria as compared to the binocularly-calibrated fixation disparity. We conclude that monocular calibration produces a physiologically more plausible fixation disparity.

This study is supported by ESF project

No.2013/0021/1DP/1.1.1.2.0/13/APIA/VIAA/001

\section{The influence of optometric and other parameters on binocular eye movements}

\author{
Remo Poffa, Roland E. Joos \\ FHNW Institute of Optometry, Switzerland \\ remo.poffa@fhnw.ch
}

Purpose: To investigate the influence of optometric and other parameters on binocular eye movement coordination, i.e. fixation disparity (FD) in reading tasks.

Methods: 20 participants (mean age $25.4 \mathrm{y}+/-4.29 \mathrm{y}$ from $21 \mathrm{y}$ to $39 \mathrm{y}, 6$ male, 14 female) were submitted to a classical optometric procedure; relevant optometric data were meas-ured. These participants underwent reading tests and eye movements were registered by using a RED500 eye tracker. Reading tests were performed under various conditions of reading distance $(50 \mathrm{~cm}$ and $100 \mathrm{~cm}$ ) and background illumination (dark and bright)..

Results: Significant optometric and non-optometric factors were found. Working distance, background illumination and their interaction are statistically significant (working distance: $\mathrm{F}=22.2$, $\mathrm{p}<.0001$; background illumination: $\mathrm{F}=10.0, \mathrm{p}=0.0017$; interaction working distance and background illumination: $\mathrm{F}=22.6, \mathrm{p}<.0001$ ). Optometric parameters were also found to be statistically significant. Best quality of statistical models was obtained by using "vergence flexibility" for modelling FD. (vergence flexibility: $F=7.54, p=0.014$ )

Conclusion: Binocular coordination is a complex function of various non-optometric and optometric parameters. If studies of binocular coordination using eye tracking methods are performed non-optometric and optometric parameters as well are important and should be accounted for. Neglecting these parameters may lead to insufficient statistical models or may mask other important parameters. 


\section{Temporal visual function in infantile nystagmus \\ Matt James Dunn, Jonathan Thor Erichsen \\ Cardiff University, United Kingdom \\ dunnmj1@cardiff.ac.uk}

Infantile nystagmus (IN) is a lifelong condition causing constant horizontal oscillations of the eyes. Despite this, individuals with IN perceive the world as stable; they do not normally experience oscillopsia. Our recent work has demonstrated that changes in the intensity of eye oscillations do not result in changes in visual acuity. Yet, people with nystagmus frequently report 'improved' vision when their eye movements are dampened (i.e. through changes in psychological state, head posture or sedation). We have developed a new test of visual function, using time-restricted visual acuity measurement to determine whether 'time to see' is an important factor in the effect of eye movements on the visual experience of those with IN. Through simultaneous gaze tracking and visual acuity measurement, we are using the test to determine the portion of the nystagmus waveform (i.e. position vs time) that corresponds to best visual acuity. By comparing the results obtained from nystagmats and normally-sighted controls, we hope to establish whether time is a limiting factor in the perception of visual information in IN. If so, then this would be consistent with patients' reports of improvement after treatments to slow their eye movements. 


\title{
Tuesday, August $18^{\text {th }}, 3.00 \mathrm{pm}-5.00 \mathrm{pm}$
}

\section{Curvature of eye movement trajectories}

\author{
A comparison of early and late measures of saccade trajectory \\ deviation \\ Luke Tudge $^{1,2}$, Torsten Schubert ${ }^{1,2}$ \\ ${ }^{1}$ Humboldt-Universität zu Berlin, Germany \\ 2 Berlin School of Mind and Brain, Germany \\ luke.tudge@hu-berlin.de
}

Saccade trajectories are sometimes curved, reflecting a process of trajectory correction that occurs during the saccade. Measures of saccade trajectory deviation that focus on the earliest part of a saccade may therefore reveal effects on pre-saccade planning that have been 'corrected away' by the time later measures are made. By extracting multiple measures of saccade deviation from previously published saccade data, as well as data from our own experiments, we were able to identify experimental manipulations that produce early saccade effects that are no longer detectable in measures of saccade landing point. We showed that distractors closer to the mid-sagittal plane distort early saccade trajectory significantly more than those further away, but that this effect is completely counteracted by correction processes. By contrast, the effect of distractor salience persists until saccade end. These findings suggest that saccade correction handles spatial effects more efficiently than feature-based effects. We offer some recommendations regarding choice of saccade trajectory measure for distinguishing between effects on pre-saccade planning and on intra-saccade correction, and make a systematic comparison of the variance and sensitivity of different measures.

\section{Spatio-temporal stimuli interactions in the visuo-occulomotor pathway: are they spatiotopic or retinotopic? \\ Geoffrey Megardon', Petroc Sumner ${ }^{1}$, Casimir J.H. Ludwig ${ }^{2}$ \\ ${ }^{1}$ Cardiff University, School of Psychology, United Kingdom \\ 2 University of Bristol, School of Experimental Psychology, United Kingdom geoffrey.megardon@gmail.com}

The primary site for stimulus related spatial interactions in oculomotor selection is normally assumed to be the superior colliculus (SC). For example, trajectory deviation away from distractor stimuli was assumed to originate from top-down distractor inhibition biasing activity in the decision field of SC intermediate layers. Recent neurophysiological work does not support this theory, and modellers have sought alternative explanations at a lower level: SC lateral interaction or even brainstem execution machinery.

In order to elucidate the level of such spatial interactions, it is important to decipher whether they are retinotopic or spatiotopic phenonmena. The SC and brainstem are retinotopic while cortical interactions could be spatiotopic.

A recent study suggests that distractor-evoked deviations are modulated by fixation activity in the SC. Here, we tested whether an initial fixation stimulus for one saccade creates deviation away in a second saccade in a spatiotopic manner. Our results favour such a spatiotopic effect and thus suggest that SC lateral connections may not be sufficient to explain saccadic deviation. 


\title{
Eye movements and perception
}

\section{Illusory motion perception and eye movements: a study of the cycloid illusion}

\author{
Hiroshi Ueda, Kohske Takahashi, Katsumi Watanabe, Yasushi Yamaguchi \\ The University of Tokyo, Japan \\ uedahi64@graco.c.u-tokyo.ac.jp
}

The cycloid illusion is a perception of a cycloidal trajectory of a point on the rim of a rolling wheel along a flat surface as a looped trajectory. Previous studies imply that the cycloid illusion originates from the failure of processing two motion components, rotation and translation, simultaneously, and this particularly occurs at the instant center of the wheel's rotation. The present study examined the relation between the illusory motion perception and eye movements by manipulating a cycloid motion stimulus. Participants observed the cycloid stimulus with or without the wheel's center and/or rim, and reported the motion trajectory. We also measured the pursuit eye movements during the trials. The addition of wheel's center causes the vector decomposition of the rotation and translation motion, whereas the addition of the rim creates an instant center on a rolling wheel. The results showed that both manipulations induced the illusory perception of the motion trajectory. Furthermore, adding an instant center of rotation also caused substantial difficulty in the trajectory perception and distorted the smooth pursuit eye movements. These results imply that the cycloid illusion might arise from at least two different processes and one of which also influences the generation of smooth pursuit.

\section{Predicting perceived visual complexity using objective image properties and eye-movement measurements}

\author{
Andreas Gartus, Jadwiga Takacs, Helmut Leder \\ University of Vienna, Austria \\ andreas.gartus@univie.ac.at
}

Visual complexity is an important concept regarding perceptual and cognitive processes. It is thought to influence detection rate, reaction time, aesthetic evaluation, etc. in many visual tasks. The goal of this work is to predict the perceived visual complexity of stimuli by combining objective computational measures (e.g. image compression rate, edge detection, etc.) with individual eye-movement statistics (e.g. fixation duration, saccade length, etc.). 63 participants were presented abstract paintings and abstract black-and-white patterns in two blocks for a $5 \mathrm{~s}$ free viewing period while recording eye-movements. After viewing each image, they were asked to rate their visual complexity on a 7-point scale. Subsequently, we calculated several objective parameters of image complexity and eye-movement statistics for each trial. All linear mixed-effects models with random intercepts for participants and stimuli using up to 2 of these parameters were calculated and ranked using AICc (Akaike information criterion corrected for small sample size). For both groups of stimuli, the best models were those that combined an objective computational measure of complexity with an eye-movement statistic. We conclude that while objective measures like image compression offer a convenient and often sufficient approximation of visual complexity, eyemovement measures can help to further improve predictions. 
Posters

\author{
Evidence for trans-saccadic fusion. \\ Celine Paeye, Thérèse Collins, Patrick Cavanagh \\ LPP - Université Paris Descartes, France \\ celine.paeye@gmail.com
}

In 1983, two studies reported the failure of trans-saccadic fusion - the integration of preand post-saccadic percepts across saccades (Bridgeman \& Mayer, 1983; O'Regan \& LevySchoen, 1983). Here we re-investigate this issue and address two problematic aspects of their stimuli. First, we tested trans-saccadic fusion with simpler objects where spatial alignment was not critical, and second, we reduced the contrast of the post-saccadic stimulus to minimize the possibility that it might mask the pre-saccadic stimulus. Participants $(n=5)$ made saccades towards a black vertical line. During the eye movement, this target was replaced with three horizontal lines that were centered on the (extinguished) pre-saccadic line. Participants indicated whether they perceived a combination of the horizontal and vertical lines or not. With the contrast of the post-saccadic lines adjusted with an adaptive staircase, participants reported seeing a superposition of pre- and post-saccadic lines on $57( \pm 5) \%$ of the trials. These findings show that, under the right conditions, a representation of the presaccadic target is available at its expected post-saccadic location and can integrate or fuse with another stimulus that is present after the eye movement, overturning the results of 1983.

\title{
Where triangles become circular: The impact of transsaccadic predictions on shape perception depends on retinal eccentricity
}

\author{
Katharina Weiß, Arvid Herwig \\ Bielefeld University, Germany \\ katharina.weiss@uni-bielefeld.de
}

Retinal position and spatial resolution of a particular object in the visual field change constantly due to saccadic eye movements. Recently, we showed (Herwig, Weiß \& Schneider, 2015, Ann N Y Acad Sci) that such saccade induced changes can be compensated for by transsaccadic predictions. Participants learned to associate differently shaped objects across saccades, - a peripheral circular object and a foveal triangular object or vice versa. Subsequently, they saccaded to a briefly presented peripheral object and judged its shape. Perceived shape was biased in the direction of previously learned associations: A triangular peripheral object that was changed to a circular object in the fovea was perceived as more circular than a transsaccadically unchanged object. Vice versa a circular peripheral object that was changed to a triangular object in the fovea was perceived as more triangular. Since spatial resolution decreases with increasing retinal eccentricity transsaccadic resolution changes are more dramatic for saccades to more eccentric locations. Thus, a transsaccadic prediction-mechanism compensating for these differences should have stronger impact at farther eccentricities. Therefore, we varied the retinal eccentricity of to be judged saccade-target shapes. As expected the impact of transsaccadic predictions on shape perception was higher at larger retinal eccentricities. 


\title{
Analyzing Gender Attribution Using Eye Tracking

\author{
Frederike Wenzlaff, Arne Dekker
} \\ University Medical Center Hamburg-Eppendorf, Germany \\ f.wenzlaff@uke.de
}

In a partial replication of the foundational work by Kessler and McKenna (1978) we investigated the relationship between gender attribution and genital attribution. To better understand the underlying decision process, we used remote eye tracking and digital reproductions of the original plastic overlays.

The stimuli, showing whole body line-drawings, ranged from exclusively male to exclusively female. The gender cues varied with regard to hair, chest, waist-to-hip-ratio, genital and body hair. For each stimulus the participants were asked to decide whether they saw a man or a woman.

In line with the results of Kessler and McKenna we found that the genital contributed immensely to the gender attribution. More participants attributed male gender when the penis was present than female gender with the vulva shown. Eye tracking revealed that most dwell time was dedicated to the head, chest and genital areas. Generally, the head was looked at significantly longer than the genital. For the congruent stimuli (all cues from the same gender) the subjective confidence ratings were higher and the reaction times shorter than for incongruent stimuli. The relative dwell time also varied across conditions, suggesting a higher relative importance of the genital for incongruent stimuli.

\section{Do eye movements reflect preference structure in more realistic scenarios?}

\author{
Kerstin Kusch ${ }^{1}$, Cölln Martin Christoph ${ }^{2}$, Helmert Jens R. ${ }^{1}$, Pannasch Sebastian ${ }^{1}$ \\ 1 Technische Universität Dresden, Germany \\ 2 Independent Researcher, Germany \\ kerstin.kusch@tu-dresden.de
}

Eye movements are used in decision research as process measures (see Orquin \& Mueller Loose, 2013 for a review). However, the resulting attentional distribution is rarely compared with estimations of structural modeling methods (see Meißner \& Decker, 2010 for an exception). Choice-based conjoint analysis (Louviere \& Hensher, 1982) represents an established method to indirectly estimate preference structure in multi-attribute decisions. We investigated, whether eye movements are concurrently valid for the measurement of preference structure in more realistic scenarios.

In the present experiment, we compared both methods (choice-based conjoint analysis and eye movement analysis) in terms of the estimated preference structure and predictive accuracy. Participants had to choose between authentic virtual 3-D yogurt cups that differed in price, flavor, fat content as well as the ease of gathering the fat content information. Eye movements were simultaneously measured. We discuss our results in the light of concurrent and predictive validity of eye movements in the measurement of preference structure especially in more realistic and complex scenarios. 
Posters

\title{
Quantification of Signal Carriers for Emotion Recognition from Body Movement and Facial Affects
}

\author{
Daniel Roth ${ }^{1,3}$, Carola Bloch ${ }^{1}$, Anne-Kathrin Wilbers ${ }^{2}$, Kai Kaspar ${ }^{2}$, Marc Erich Latoschik ${ }^{3}$, \\ Gary Bente ${ }^{1}$ \\ ${ }^{1}$ Media and Communication Psychology, Department of Psychology, University of Cologne, Cologne \\ ${ }^{2}$ Social and Media Psychology, Department of Psychology, University of Cologne, Cologne \\ ${ }^{3}$ Human-Computer Interaction, Institute for Computer Science, University of Würzburg, Würzburg \\ daniel.roth@uni-koeln.de
}

Nonverbal expressions of emotions in the human face and body play an important role in social interactions. Regarding affective human-computer interfaces and the use of nonverbal cues in computer-mediated communication, knowledge about the contribution of different nonverbal channels to emotion recognition is essential. The current study analyzed the relative impact of emotional expressions in faces and bodies of avatars on visual attention and emotion recognition. Avatar animations of expressive body movements were based on motion capture data from actors portraying either anger or happiness. A pre study was conducted to select expressions which were either perceived as anger, happiness or neutral. We systematically combined facial expressions and expressive movements by using blend shapes for facial animation resulting in congruent and incongruent face-body combinations. 68 participants watched the resulting videos and rated the perceived emotion. During stimulus presentation gaze behavior was recorded with a SMI RED500 eye tracker. Using dynamic regions of interest we calculated dwell times and number of fixations in the face, head and body region. Results indicate that humans prioritize the face and head region as an information source emotion recognition. This priority is visible in the visual attention patterns as well as in the explained judgment variance.

\section{Object substitution masking across saccadic eye movements Karl Verfaillie, Bart Michiels, Maarten Demeyer, Filip Germeys Laboratory of Experimental Psychology, Belgium Karl.Verfaillie@ppw.kuleuven.be}

Object substitution masking (OSM) has been used extensively to study the role of feedforward and feed-back signals in visual perception. While an extensive literature exists on OSM within fixations, as a phenomenon it has not yet been investigated across saccades. In the present study we investigate whether OSM also occurs transsaccadically. Additionally, we relate re-entrant processing theories of OSM to the vVisual aAnalog component of transsaccadic memory. In the first eExperiment 1, we employ a transsaccadic change detection paradigm with the addition of postsaccadicin combination with an OSM masks procedure. The results show a clear transsaccadic OSM effect. TAdditionally, two follow-up experiments suggest that transsaccadic OSM may appear to have different properties than within-fixation OSM. SMore specifically, transsaccadic OSM seems to be insensitive to the lack of common onset between the target and the OSM-mask, and to previewing the mask before target onset. In contrast, in a within-fixation context these manipulations have been reported to reduce OSM in a within-fixation context. We propose that the added issue of establishing visual stability in a transsaccadic paradigm might explain the difference in properties between within-fixation and across-fixation both types of OSM in a within-fixation and a transsaccadic context. 


\title{
Eye-tracking methods
}

\section{An evaluation of velocity-based saccade detection in mobile eye tracking}

\author{
Ali Abedian-Amiri, Hans A. Trukenbrod, Ralf Engbert \\ University of Potsdam, Germany \\ abediana@uni-potsdam.de
}

There is an increasing interest in studying eye movements in dynamic natural tasks. Due to recent advances in mobile eye-tracking technology, sampling rates of up to $60 \mathrm{~Hz}$ and high spatial resolution are now available. However, fixation-based event detection is currently the state-of-the-art procedure of data preprocessing for sampling rates below $200 \mathrm{~Hz}$. Here we show that velocity-based saccade detection algorithms can be used for eye-tracking data of intermediate temporal resolution $(60 \mathrm{~Hz})$. Eight participants performed a variety of natural tasks wearing mobile eye-tracking glasses. Saccades and fixations were identified with a (micro)saccade detection algorithm (Engbert \& Kliegl, 2003; Engbert \& Mergenthaler, 2006). Based on this algorithm, we identified saccade amplitude and fixation duration distributions that are qualitatively comparable with those obtained from high-resolution eye tracking ( $500 \mathrm{~Hz}$ and above). The use of velocity-based saccade detection algorithms seems to be a promising approach even for devices with intermediate temporal resolution. We conclude that saccade detection in intermediate temporal resolution data is conceptually similar to microsaccade detection in high-resolution laboratory eye-tracking devices.

\section{Defining Areas of Interest for the Dynamic Web Pages \\ Robert Moro, Jakub Daraz, Mária Bieliková \\ Slovak University of Technology in Bratislava, Slovak Republic robert.moro@stuba.sk}

Although most of the Web pages nowadays has a dynamically changing content, the existing gaze analysis tools support mainly static content by allowing definition of areas of interests (AOI) using polygons. This presents serious limitation if web elements change position, size or when they are overlaid by other elements.

We developed a browser extension that enables a specification of AOIs as web page elements by simply clicking on the elements. It is possible to fine-tune the selection by modifying the underlying XPath string (e.g., by selecting all the elements with the same value of a class attribute). The gaze coordinates are mapped to a specific element the user looks at. The element is uniquely identified by an XPath string which is matched with one (or none) of the predefined AOIs.

Our main contribution lies in the shift from the physical representation of the gaze areas to the logical elements (e.g. advertisements, menu, navigation, widget) which can be identified (and the gaze data aggregated) throughout the whole Web portal or system. We account also for the possible inaccuracy of the eye trackers by allowing additional space around the defined areas.Current implementation supports Tobii X2-30, X2-60, EyeX and EyeTribe. 
Posters

\title{
An upgraded program for statistical analysis of eye movement data: The transition patterns
}

\author{
John J. H. Lin, Grace M. Y. Hsieh, Sunny S. J. Lin \\ National Chiao Tung University, Taiwan, Republic of China \\ john.jrhunglin@gmail.com
}

Lin and Lin (2013) developed an integrative program for eye movement data analysis. The previous version targeted at fixation-based data. During a learning scenario, the ability to integrate information among a set of areas is an indicator to evaluate learning performance. Therefore, this upgraded program added the saccade-based function, the transition pattern, which quantifies the attention shifts among specific AOIs. Two indicators were added to the program, including numbers of transition and transition length. Before analyzing a dataset, researchers decide a set of specific AOIs and the transition pattern is unidirectional or bidirectional. Then, the program will merge the online and offline data, and reconstruct them into a single dataset. After completing the analysis, the output will show the descriptive statistics and bar charts of each group. Results of the analysis of variance for non-parametric dependent sample are provided as well. Researchers can add new modules, such as Linear mixed model, based on their needs. The results are exported as *.docx and *pdf. We have applied the program in a learning experiment to investigate whether two groups with different learning performance (High v.s. Low) were different in transition patterns. Results indicated no significant difference between two groups.

\section{Easy as Py: Eye Tracking with OpenSesame and PyGaze}

\author{
Sebastiaan Mathôt ${ }^{1}$, Lotje van der Linden ${ }^{1}$, Eduard Ort $^{2}$, Daniel Schreij ${ }^{2}$, Stefan \\ Van der Stigchel ${ }^{3}$, Edwin S. Dalmaijer ${ }^{4}$ \\ ${ }^{1}$ Aix-Marseille University, CNRS, LPC UMR 7290, Marseille, France \\ 2 Dept. of Cognitive Psychology, VU University, Amsterdam, Netherlands \\ 3 Dept. of Experimental Psychology, Helmholtz Institute, Utrecht University, Utrecht, Netherlands \\ ${ }^{4}$ Dept. of Experimental Psychology, University of Oxford, Oxford, United Kingdom \\ s.mathot@cogsci.nl
}

Developing eye-tracking experiments can be difficult and time consuming. Here we present two software packages, OpenSesame and PyGaze, that make development easier and faster. OpenSesame is an experiment builder with an intuitive graphical user interface, and support for Python scripting. PyGaze is a Python library that provides a high-level interface for communicating with eye trackers (EyeLink, EyeTribe, SMI, and Tobii). PyGaze integrates with OpenSesame through a set of graphical plug-ins. Together, OpenSesame and PyGaze allow unexperienced users to quickly implement basic eye-tracking experiments, including gaze-contingent paradigms. Advanced users can include Python scripting to create complex experiments. OpenSesame and PyGaze are freely available under an open-source license, are under active development, and are supported by a large community. During the poster presentation, an eye tracker will be available to demonstrate the software. 


\title{
The method of lie detection during face recognition using eye tracking
}

\author{
Artem Kovalev, Elizaveta Luniakova, Galina Y. Menshikova \\ Lomonosov Moscow State University, Russian Federation \\ artem.kovalev.msu@mail.ru
}

Recently new algorithms for lie detection have been developed, including those based on the eye-tracking technology. It was shown that the count and duration of fixations, blinks and saccades could be used as indicators of information deception. In our study we investigated the dwell time and the number of revisits on the target for lie detection. Eye movement parameters were recorded in the face recognition task. Three types of photos were used: the familiar face, which the subject should recognize (type I); the familiar face, the subject should lie about (type II); unfamiliar faces (type III). In each trail 9 photos grouped in a $3 \times 3$ matrix were presented among which were either 8 unfamiliar and 1 familiar faces or all faces were unfamiliar. The results showed that the dwell time and the number of revisits for unfamiliar faces were significantly lower than for familiar faces. The same indicators for familiar faces of type II were significantly lower than for type I. The obtained data allow considering the dwell time and the number of revisits as indicators, which may improve deception detection methods in combination with other eye movement characteristics.

The work was supported by RFBR grant No. 13-07-00834

\section{Comparing Eye-tracking Data Using Machine Learning}

Michal Barla, Miroslav Šimek, Mária Bieliková

Institute of Informatics and Software Engineering, Slovak University of Technology in Bratislava, Slovak Republic

michal.barla@stuba.sk

Current approaches to eye tracking studies rely upon manually defined areas of interest or manually designed features combined with machine learning algorithms. However, these do not scale very well when working with several eye trackers in parallel. In our case, we have twenty X2-60 Tobii eye trackers employed within ordinary courses, which leads to vast amounts of data. We propose a generic way how to learn an efficient abstraction of a user session, which is especially suitable for comparing, clustering and categorizing user sessions.

Our approach is based on unsupervised machine learning, specifically Restricted Boltzmann Machine (RBM), which is presented with fragments of user sessions in form of heat maps capturing spatial (pixel coordinates) and time (pixel intensity) information. RBM is able to find its own features to make efficient session abstraction in the context of other user sessions.

We developed a set of applications that preprocess the eye-tracking data, train the RBM, acquire data abstractions from trained RBM and process them into most common patterns over time for each individual user session. Subsequently we are able to cluster sessions into groups and find those, where users did not behave as expected. These outliers are chosen for closer, manual inspection. 
Posters

\title{
A Model for Analyzing the Role of Camera Parameters in the Clinical Assessment of Eye Movements
}

\author{
Mehrdad Sangi ${ }^{1}$, Benjamin Thompson ${ }^{2,3}$, Cindy Xiaopeng Guo ${ }^{2}$, Jason Turuwhenua ${ }^{1,2}$ \\ 1 Auckland Bioengineering Institute, University of Auckland, New Zealand \\ 2 Department of Optometry and Vision Science, University of Auckland, New Zealand \\ ${ }^{3}$ School of Optometry and Vision Science, University of Waterloo, Canada \\ msan223@aucklanduni.ac.nz
}

There is clinical interest in low cost consumer-grade devices such as webcams and smartphone cameras for the measurement of visual function. We investigated whether a webcam (30 frame per second) was sufficient to measure the velocity characteristics of optokinetic nystagmus (OKN), an involuntary sawtooth movement of the eye that may allow for rapid and objective clinical measurement of vision. OKN consists of both smooth pursuit (slow) and saccadic (fast) eye movements, each of which can provide important information relating to visual functions such as visual acuity. We developed a geometric model of the eye and camera, which took as input the displacement signal obtained from a commercial grade eye tracking system. Our experimental results confirmed that consumer-grade cameras could measure the smooth pursuit phase of OKN (30 fps), but at least $210 \mathrm{fps}$ was needed for reliable measurement of the saccadic phase. Current improvements in smartphone camera frame rates may allow for the use of these devices to quantify the temporal properties of the saccadic phase of OKN in clinical practice. Our model can be used to analyze the role of camera intrinsic and extrinsic parameters as an assistive tool for designing eye tracking experiments and calibration procedures.

\section{Effect of wearing glasses on eye tracking measurements Filip Děchtěrenko ${ }^{1}$, Jiří Lukavský ${ }^{2}$, Kenneth Holmquist ${ }^{3}$ \\ ${ }^{1}$ Faculty of Mathematics and Physics, Charles University in Prague, Czech Republic 2 Institute of Psychology, The Czech Academy of Sciences, Czech Republic \\ 3 Humanities Laboratory, Lund University, Sweden filip.dechterenko@gmail.com}

In almost every eye tracking study participants with myopia are measured sometimes with or without the glasses. It is unclear whether the loss of sharp vision leads to differences in eye movements. In our pilot study we measured eye movements in Multiple object tracking experiment, while the participants $(\mathrm{N}=8)$ were asked to put their glasses on and off between blocks. Each experimental trial was presented twice ( $2 \times 2$ design: first/second presentation with glasses on/off) and we compared to what the scan patterns were similar. Similarity of scan pattern was computed by correlating spatiotemporal map for each pair of scan patterns.

The results of within-subject ANOVA showed that putting glasses on or off does affect the similarity of scan patterns $(\mathrm{p}<.05$, eta-squared $=.29)$. People wearing glasses in both presentations are more likely to repeat their eye movements compared to the situation when both repetitions were tracked without glasses $(\mathrm{p}<.05)$. We plan to add more participants into the study and include the number of diopters into the analysis. 


\title{
Continuous 3D Recovery of Human Gaze using Multisensory Pose Tracking
}

\author{
Lucas Paletta, Michael Schwarz, Stefan Ladstätter, Martin Pszeida \\ JOANNEUM RESEARCH Forschungsgesellschaft $\mathrm{mbH}$, Austria \\ lucas.paletta@joanneum.at
}

Recent studies on automated gaze recovery in 3D with eye tracking glasses applied purely image based pose estimates (Santner et al., 2013, Paletta et al., 2013) in a previously acquired model of the environment, without the use of any artificial landmarks in the field of view. The applied methodology enables high precision when users move in slow motion. However, under real world conditions, users frequently move their head rapidly and thus generate imagery with image blur which complicates automated analysis. This work presents multisensory recovery of 3D gaze using a motion sensor attached to eye tracking glasses in order to complement image based pose estimation using a particle filtering approach (Ristic et al., 2004). We apply a quality measure in terms of image sharpness estimation for the appropriate control of filter weight reassignments under varying operating conditions. An experimental indoor study reports an increase of $8.7 \%$ in the high precision coverage of 3D measurements, with an accuracy of $3.92 \mathrm{~cm}$ for $94.4 \%$ of the captured data. Furthermore, we achieve a truly continuous operation of gaze recovery in contrast to the purely image based approach that temporarily suffers from outliers. 
Posters

\title{
Microsaccades
}

\section{Contrast-Dependent Neural Responses to Microsaccades in Primary Visual Cortex (V1)}

\author{
Jie Cui ${ }^{2}$, Stephen L. Macknik ${ }^{1,2}$, Susana Martinez-Conde ${ }^{1,2}$ \\ ${ }^{1}$ State University of New York (SUNY) Downstate Medical Center, United States of America \\ ${ }^{2}$ Barrow Neurological Institute \\ jie@neuralcorrelate.com
}

Even during attempted fixation, we produce eye movements. These include microsaccades, or small-magnitude saccades that occur once or twice a second. Microsaccades evoke strong modulation of neural responses in area V1, but the nature of this neural activity is not well understood. Here we show the modulation of microsaccade-triggered single-neuron activity in V1 as a function of stimulus contrast. Three monkeys were trained to fixate a small red cross, while their eye movements were recorded using the scleral search coil technique. Visual stimuli consisted of oriented Gabor patches, presented at different contrast levels. The Gabor stimuli were centered over the cells' classic receptive fields (RFs), with orientations matching the preferred orientation of each neuron. As control conditions, we also recorded the neural activity when the monkeys were presented with uniform stimulation ("Uniform" condition), or when the monkey chamber was completely dark (except for the small LED fixation target; "Dark" condition). We found a prominent tri-phasic modulation of neural responses (i.e. an initial enhancement followed by decreased responses and a later enhancement). Our preliminary results indicate that the initial enhancement is retinal in origin, and that the later enhancement may be due to visual stimulation outside the classic RF.

\section{The effect of music reception on microsaccade rate and pupil dilatation}

\author{
Elke B. Lange ${ }^{1}$, Petra Sinn ${ }^{2}$ \\ ${ }^{1}$ Max-Planck-Institute for Empirical Aesthetics, Germany \\ 2 University of Potsdam, Germany \\ elke.lange@aesthetics.mpg.de
}

Fixational eye movements can be differentiated into microsaccades, drift and tremor. There is growing evidence that microsaccade rate index cognitive load as well as recruitment of attentional resources. In addition, pupil size has been reported to relate to mind-wandering episodes. We chose both measurements to exploratory tackle the mind set of being absorbed when listening to music. Data were collected in a sound proof laboratory with high-end audio equipment and an EyeLink 1000 (SR Research). Twenty-four participants listened to 60 segments of music from a broad selection of different musical styles, e.g. Electronic Music, Pop, Funk, Soul, Rock, Metal, Jazz, and Classic. As a control we recorded eye movements and pupil size in silence. Music was rated for felt arousal, valence, absorption, preference, and familiarity. These ratings served as a "subjective" level of description. In addition, the acoustic features of the music excerpts were extracted by the MIR toolbox. Such features represent an "objective" level of music description, e.g., brightness, roughness, energy, puls clarity, tempo, pitch height. Data were statically analyzed using continuous predictors in a mixed modeling approach. First analyses indicate a complex pattern of effects of the subjective ratings as well as the acoustic descriptors on microsaccade rate. 


\title{
Reading
}

\author{
Eye Movement Control in Turkish: Word-Length and \\ Morphological-Complexity Effects on Fixation Landing Positions \\ Cengiz Acartürk, Figen Beken, Bilal Kirkici \\ Middle East Technical University, Turkey \\ acarturk@metu.edu.tr
}

The goal of the present study is to identify the effect of word length and morphological complexity on eye movement parameters in Turkish reading. Turkish is an agglutinative language and is known for its rich morphological structure. We collected eye movement data from 24 native Turkish speakers, who read 120 nine-word sentences selected from a two-million word written corpus for Turkish (viz. METU Corpus). The words covered a wide variety in terms of the number of letters $(\mathrm{M}=5.6, \mathrm{SD}=2.48)$, the number of suffixes (from 0 to three), as well as frequency. The analyses of the effect of word length and the effect of morphological complexity on first Fixation Landing Positions (FLPs) were conducted using linear mixed models (LMMs). The model included the variance components of participants and items. The results showed that both word length $(b=1.07, \mathrm{SE}=.08, \mathrm{t}=13.38)$ and number of suffixes $(b=.1, S E=.03, t=3.39)$ have significant effects on Fixation Landing Positions, exhibiting a FLP tendency to the right of the word center. Moreover, a significant interaction was observed between these variables $(b=-.15, \mathrm{SE}=.04, \mathrm{t}=-3.85)$. These findings will be discussed from the perspective of the analysis of parameters that influence eye movement control in reading.

\section{Referential ambiguity resolution: an eye-tracking study with reference to Russian \\ Veronika Prokopenya, Aleksandra Talanina, Daria Chernova \\ St.Petersburg State University, Russian Federation veronika.info@gmail.com}

We investigated the processing of Russian referentially ambiguous and unambiguous texts, consisting of two sentences. The first sentence contained two referents, and the second sentence contained a personal pronoun, that could refer either to both referents - in ambiguous context (when referents were of the same grammatical gender), or to only one referent - in unambiguous context (when referents differed by gender). If there are few good antecedent candidates, pronouns tend to refer to the entities that play prominent role in the discourse. This prominence is provided by various factors related to grammatical structure, information structure, and focus of attention. The analysis of the oculomotor behavior let us reveal the particular role of each factor. The participants, Russian native speakers, had to read sentences and then answer the questions about pronoun comprehension. We found an increase in reading time of ambiguous sentences compared to unambiguous ones. Furthermore, participants produced more regressions out of the sentence with pronoun to the previous sentence with potential referents in ambiguous condition. The analysis of the regressions areas and of the correlation with participants' answers let us argue that the pronominal reference assignment is rather driven by information-structural than by grammar-structural factors. 
Posters

\title{
Contextual predictability and phonological preview benefit in reading Chinese sentences
}

\author{
Jie-Li Tsai ${ }^{1}$, Chia-Ying Lee ${ }^{2}$ \\ 1 National Chengchi University, Taiwan, Republic of China \\ 2 Academia Sinica, Taiwan, Republic of China \\ jltsai@nccu.edu.tw
}

The present study aimed to examine whether the phonological preview benefit can be modulated by the contextual predictability. Studies have reported the word predictability effect on word skipping rate and suggest that contextual influence can occur early in parafoveal processing. However, it is unclear whether the early contextual influence facilitates word processing at the form and phonological levels. One experiment manipulated contextual predictability (predictable vs. unpredictable) and preview type (identical, orthographic, phonological, or unrelated to target), using the boundary paradigm in a sentence reading task. The results replicated the previous study showing the phonological preview benefit in reading Chinese. The contextual predictability effect was observed in early eye movement measures and it interacted with the preview effects. Moreover, the phonological preview benefit was enhanced when the word was predictable from the context. The nature of topdown contextual influence on early lexical processing will be discussed.

\author{
Eye movements of two extremely fast readers \\ Ralph Radach ${ }^{1}$, Christian Vorstius ${ }^{1}$, Thomas Lüttke ${ }^{1}$, Anne Friede ${ }^{1}$, Ronan Reilly ${ }^{2}$ \\ ${ }^{1}$ University of Wuppertal, Germany \\ ${ }^{2}$ National University of Ireland, Maynooth \\ radach@uni-wuppertal.de
}

We describe eye movements of two individuals reporting mastery of three different speed reading strategies, fast normal reading with internal articulation (FNR), line-by-line optical reading without articulation (LOR) and areal optical reading (AOR) including information acquisition from multiple lines. Both subjects read extended passages of text for comprehension and completed a visual span task (Legge, 2006) to determine their performance in extrafoveal letter processing.

Results indicated that for one participant reading speed increased from 376 words per minute (wpm) in FNR to $508 \mathrm{wpm}$ in LOR and $526 \mathrm{wpm}$ in AOR. For the second participant reading speed changed from $412 \mathrm{wpm}$ to $570 \mathrm{wpm}$ in LOR and $936 \mathrm{wpm}$ in the AOR mode. Comprehension scores remained within the range shown by participants in a group of normal readers. Eye movement patterns appeared quite regular, with long forward saccades and almost no refixations and re-reading of prior words. Distributions of word viewing time measures were markedly shifted towards shorter durations. Both participants showed exceptional performance in the visual span task, especially with orthographically regular letter triplets. Together with superior mental speed found in additional assessments, this extremely efficient letter decoding may help explaining the extraordinary performance of the two speed readers 


\section{The Morphological Structure of Compound Words influences Parafoveal Processing in Chinese Reading}

Lei Cui ${ }^{1}$, Denis Drieghe ${ }^{2}$, Guoli $\mathrm{Yan}^{3}$, Xuejun $\mathrm{Bai}^{3}$, Hui Chi ${ }^{3}$, Simon P. Liversedge ${ }^{2}$

${ }^{1}$ Shandong Normal University, People's Republic of China

2 University of Southampton, UK

3 Tianjin Normal University, China cuilei_cn@163.com

In an eye movement boundary paradigm experiment (Rayner, 1975) we compared parafoveal preview benefit during the reading of Chinese sentences. The target word was a 2-character compound that had either a noun-noun or an adjective-noun structure each sharing an identical noun as the second character. The boundary was located between the two characters of the compound word. Prior to the eyes crossing the boundary the preview of the second character was presented either normally or was replaced by a pseudocharacter. Previously, Juhasz, Inhoff and Rayner (2005) observed that inserting a space into a normally unspaced compound in English significantly disrupted processing and that this disruption was larger for adjective-noun compounds than for noun-noun compounds. This finding supports the notion that, at least in English, for adjective-noun compounds, the noun is more important for lexical identification than the adjective, while for noun-noun compounds, both constituents are similar in importance for lexical identification. Our results showed that the pseudo-character preview was more disruptive for the adjective-noun compounds than the noun-noun compounds in Chinese indicating that parafoveal processing can be influenced by the morphological structure of the currently fixated character.

\section{Eye movement in comprehending infographics with different color arrangement \\ Tsuei-Ju Hsieh ${ }^{1}$, Yen-Chih Chen ${ }^{2}$ \\ ${ }^{1}$ Chinese Culture University, Taiwan, Republic of China \\ 2 Realtek Semiconductor Corp. tracy.tjhsieh@gmail.com}

In infographics design practice, color is an essential feature that increases clearness and attractiveness. To investigate the color effect in viewers' infographics comprehending behavior, we conducted a between-subject experiment. Eighteen infographics were adopted and four conditions were manipulated, black-and-white, red-marked, congruent-colorful, and incongruent-colorful. Thirty participants were recruited in each condition and their eye movement were tracked by Eyelink 1000 at $500 \mathrm{~Hz}$. In a trial the participant had twelve seconds to look at a graphic and then answered four multiple-choice questions with respect to the graphic contents. The resulting eye movement data are first mapped to a set of saliency maps based on some selected thresholds. We conducted receiving operating characteristic (ROC) analyses based on the generated saliency maps, and identified the similarities of gazing pattern between four conditions for each infographic. Also, the correction rate and eye movement metrics were compared across conditions. The red-marked condition resulted in higher correct rate, while incongruent-colorful resulted in lower correct rate, less fixation and saccade numbers, and more blinks. Generally, color arrangement affects the eye movement pattern in comprehending infographics, and has marginal significant effect on the correct rate. 
Posters

\section{Lexico-syntactic interactions in second language sentence processing: What can eye-movements reveal?}

Ana Paula Soares ${ }^{1}$, Helena Oliveira ${ }^{1}$, Marisa B. Ferreira ${ }^{2}$, Montserrat Comesaña ${ }^{1}$, António Filipe Macedo ${ }^{2}$

${ }^{1}$ Human Cognition Lab, CIPsi, School of Psychology, University of Minho, Portugal

${ }^{2}$ Vision Rehabilitation Lab, Centre of Physics and Optometry, University of Minho, Portugal asoares@psi.uminho.pt

In Psycholinguistics there is extensive evidence showing that bilinguals activate lexical representations from both languages in a non-selective way, even in sentence contexts. However, what is still unclear is the extent to which the lexical representations interact with syntactic representations during second language (L2) sentence comprehension (especially in languages where the parsers operate in opposite ways such as Portuguese and English) and how this interaction is modulated by L2 proficiency. This work explored the lexicosyntactic interactions in the processing of temporarily ambiguous sentences involving a complex noun phrase followed by a relative clause [NP-V-NP1-of-NP2-RC] in L2 by nativespeakers of Portuguese (L1) who are intermediate and advanced learners of English (and their respective controls), using both an off-line (sentence completion) and an on-line (silent reading) task while monitoring eye-movements. In each of these tasks the cross-language similarity (i.e. the cognate status) of the nouns embedded in the complex noun phrase was manipulated in four experimental conditions: cognate-cognate (both NP1 and NP2 contain a cognate word); cognate-noncognate (NP1 contains a cognate and NP2 a noncognate); noncognate-cognate (NP1 contains a noncognate and NP2 a cognate); and noncognatenoncognate (both NP1 and NP2 contain noncognates). Results on RC preference and eyemovements measures are compared and discussed. 


\title{
Reading short stories: Repetition Effects and Semantic Distance Effects on Eye Movements
}

\author{
Juan E. Kamienkowski ${ }^{1,2,3}$, Bruno Bianchi ${ }^{1,3}$, Maria J. Carbajal ${ }^{1}$, Facundo Carrillo ${ }^{1,2}$, \\ Mariano Sigman ${ }^{1,4}$, Diego Fernandez Slezak ${ }^{1,2}$, Diego E. Shalom ${ }^{1,3}$ \\ ${ }^{1}$ Laboratory of Integrative Neuroscience Buenos Aires, Argentine Republic \\ ${ }^{2}$ Laboratory of Applied Artificial Intelligence, Computer Science Dept, School of Exact and Natural \\ Science, University of Buenos Aires, Argentine Republic \\ 3 Physics Dept, School of Exact and Natural Science, University of Buenos Aires, Argentine Republic \\ ${ }^{4}$ Laboratory of Neuroscience, University Torcuato Di Tella, Buenos Aires, Argentine Republic \\ juank@df.uba.ar
}

When we read, we continuously predict the follow words to integrate information and direct future eye movements. Thus, that Predictability has become one the most important variables when explaining human behavior and information processing during reading. Some of the building blocks of Predictability could be the local frequency of the word -i.e. the number of repetitions within the text- or semantic relations with the context.

We recorded eye movements from participants reading long texts ( 3000 words), and estimated cloze-task predictability in a massive web-based experiment. We analyzed fixation durations and predictability in terms of the above mentioned building blocks: We found significant effects of repetition of the word but not it's lemma. Semantic relations were estimated using computational measures as Latent Semantic Analysis or word2vec. We showed that those computational-distance between the word of interest and an adequate number of previous words captures some semantic aspects of the predictability.

In summary, we showed that is possible to unbundle many components of cloze-task predictability, paving the way to understand the actual cues used to make the prediction, and to estimate it from text for future experiments and applications without the need of a clozetask experiment.

\section{Reading eye movement behaviour in a large cohort of 9-year old children}

\author{
Suzanne C. Louwen ${ }^{1,2}$, Rick van der Vliet ${ }^{1}$, Hieab H. Adams ${ }^{2}$, Henning W. Tiemeier ${ }^{2}$, \\ Jos N. van der Geest ${ }^{1}$ \\ 1 Neuroscience, Erasmus MC, The Netherlands \\ ${ }^{2}$ Child Psychiatry / Epidemiology, Erasmus MC, The Netherlands \\ suzannelouwen@hotmail.com
}

Eye movement behaviour during reading consists of a distinctive pattern of fixations and saccades. Ample research suggests reading abilities are related to the quality of eye movement patterns, reflected by the duration of fixations and the direction and lengths of saccades. This relationship has been observed in children with dyslexia compared to healthy controls. Yet, little is known about the relationship between the development of reading skills and eye movements behaviour in the general population. We currently investigate eye movement behaviour during reading in a large subsample $(\mathrm{N}>600)$ of 9-year old children with different reading abilities participating in the Generation R cohort, a longitudinal study examining the development of about 10,000 children in Rotterdam, The Netherlands. First, we examine fixation behaviour when children perform a 1-minute word-reading task and correlate fixation times with reading performance. Next, we look at the pattern of fixations and saccades in a natural reading task. Individual determinants of reading behavior like school performances, gender, age and ethnic background will be taken into account. Our results will contribute to the evidence relating reading ability and eye movement patterns. 
Posters

\title{
The effect of horizontal movement of text on lexical and sentence-level processing during reading
}

\author{
Hannah Harvey ${ }^{1}$, Hayward J. Godwin ${ }^{2}$, Gemma Fitzsimmons ${ }^{2}$, Simon P. Liversedge ${ }^{2}$, Robin \\ Walker $^{1}$ \\ ${ }^{1}$ Royal Holloway University of London, United Kingdom \\ 2 University of Southampton \\ hannah.harvey.2010@live.rhul.ac.uk
}

Horizontally scrolling dynamic text (e.g. news tickers) presents a theoretically interesting paradigm due to the conflict between tracking the text moving right-to-left and the normal left-to-right saccades made during reading. Here we report findings from three experiments investigating how this impacts oculomotor behaviour and linguistic processing during reading. Experiment 1 investigated manipulation of two word-level characteristics, length and frequency, demonstrating a similar facilitation effect (shorter fixation durations and increased word skipping for shorter and more frequent words) with dynamic text as is found with static text. Experiment 2 examined sentence-level linguistic processing using a predictability manipulation (high versus low predictability of target words based on preceding context). We found no early facilitation of word identification for highly predictable target words, suggesting that on-line computation of sentence meaning was impaired for the dynamic text format. Experiment 3 further examined reading comprehension in a sustained reading task and showed that dynamic text reduced comprehension. Together, these findings indicate that whilst lexical processing of individual words may not be impaired, integration of information across words and sentences may be disrupted by dynamic text. Such disruption is likely due to the reduced opportunity for regressive saccades (for reinspection of text) and higher cognitive load.

\section{When Words Are Changing Places: Eyetracking Reveals Control Strategies}

\author{
Chris Lange-Küttner, Markowska Monika \\ London Metropolitan University, United Kingdom \\ c.langekuettner@londonmet.ac.uk
}

We assessed free word recall in mono- and bilinguals with three word lists each once repeated (Exp. 1, N=45, Exp.2 N=40). In Exp. 1 bilinguals showed a comparably higher improvement rate of their recall score in repeated blocks when target word and rhymed distracter had changed places. We hypothesized that this occurred because of more flexible lateralized eye movements and analysed in Exp. 2 spatial flexibility with an eye tracker. The area of interest (AoI) was the left versus right field of the screen. The repetition advantage for bilinguals could be replicated for women only, but not for men who all improved word recall in repeated blocks. Accordingly, a significant block by field by language by gender effect was found for revisiting places in women only. In women, in Block 1 the left side was revisited more often, in Block 2 the right side was revisited more often, while in Block 3 monolingual women showed more flexibility and revisited again more often the left side, while bilingual women revisited again more on the right side. Men showed no lateralized revisiting. Thus, eyetracking analysis revealed a strategy of women trying to control the place swapping, while men made no such effort. 


\title{
Effects of font size and spacing on Chinese reading the newspaper material in urban low-age senior citizens
}

\author{
Jie $\mathbf{W u}^{1}$, Haiyu Cheng ${ }^{2}$, Mengxuan $\mathbf{W u}^{3}$ \\ 1 Academy of Psychology and Behavior, Tianjin Normal University \\ 2 Academy of Psychology and Behavior, Tianjin Normal University \\ 3 College of Foreign Language, Nankai University \\ babaluosha@163.com
}

With the aging process intensified in China, $\mathrm{m}$ attentions are paid to the demand for elderly people. Reading is one of the most important cognitive activities for the elders and lots of studies focus on it. However, few studies have focused on the elders' daily reading, especially the front size and line-spacing is still a question to be explored.

In present study, an eye tracker was used to investigate the influence of character size and line-spacing on the Chinese passage reading for elder adults. 32 participants (age: 60-69 years old) were asked to read8simple passages with character size ( 20 pounds \& 24 pounds) and line-spacings (single-spaced \& 1.5-spaced). Then, they had to answer two multiplechoice questions. Participants' eye movements were recorded with an iView X RED manufactured by Senso-Motoric Instruments (SMI).

The results were as follows: (1) When larger-size characters were presented, the fixation count increased, average fixation duration decreased, saccade length shorted and the reading speed accelerated significantly. (2) When under the wider line spacing condition, elders read faster than reading the passages with narrow line. These findings suggested that larger characters and wider line-spacing can improve elders' subjective reading experience greatly.

\section{Using eye-tracking to explore formulaic sequences: Some methodological challenges}

\author{
Gareth T. Carrol, Kathy Conklin \\ University of Nottingham, United Kingdom \\ gareth.carrol@nottingham.ac.uk
}

In this poster we explore the methodological challenges that exist when we apply eyetracking to the processing of multiword units. Such "formulaic" sequences (e.g. idioms, such as "kick the bucket") necessarily span several words, so traditional eye-tracking measures, as they relate to single word processing, become less straightforward to apply. At the same time, formulaic sequences are considered to exist as "whole units", at least on some level, however treating them as single items eliminates much of the fine-grained detail that eye-tracking can provide.

Drawing on several of our own studies, we demonstrate the issues that have become apparent in this area. We discuss studies of idiom processing using native and non-native speakers, and demonstrate how a combination of phrase and word-level analysis, and early and late eye-tracking measures can best elucidate the locus and nature of the formulaic advantage.

We also demonstrate how different measures can be used to explore the different facets of formulaic language: knowledge and recognition of a fixed form vs. knowledge of a holistic, phrase level meaning, and the ability to integrate this into a wider sentence context. We conclude by proposing a "hybrid" method of analysis as the best way to proceed. 
Posters

\title{
Social influences
}

\section{Eye movements in sequential line-ups for suspects with distinctive features}

\author{
Adrian von Mühlenen ${ }^{1}$, Marie Luisa Schaper ${ }^{2}$, Daniel Steinmann ${ }^{2}$, Kimberly Wade $^{1}$ \\ 1 University of Warwick, United Kingdom \\ 2 Heinrich-Heine-Universität Düsseldorf \\ a.vonmuhlenen@warwick.ac.uk
}

In the creation of fair line-ups for suspects with distinctive features (such as tattoos, birth marks, rashes) the police either replicate the distinctive feature on the foils' faces or conceal the feature on the suspect's face to prevent these suspects from standing out. Research on simultaneous line-ups suggests that replication results in better performance than does removal. However, there is no empirical evidence for a similar pattern in sequential lineups, although many countries prefer these over a simultaneous presentation. The current study tested replication versus removal of distinctive features (compared to a "do-nothing" control condition) in sequential line-ups. Eye movements were recorded during study and test phase. Results showed better identification in the replication and control condition than in the removal condition. In target-absent trials, foil identification rate was also reduced in the removal condition. However, participants showed a high conditional probability of choosing the distinctive foil in the control condition (given an identification was made). Eye movement analysis further suggest a link between identification performance and fixation rate/duration of faces with and without distinctive feature. Replication and do-nothing seem to be eligible options when a suspect has a distinctive feature, however, do-nothing might be unfair to innocent suspects.

\section{Appealing or threatening images alter gaze behavior with attractive faces}

\author{
Jürgen Goller, Aleksandra Mitrovic, Helmut Leder \\ University of Vienna, Austria \\ juergen.goller@univie.ac.at
}

The human sense for facial attractiveness is often related to functional and behavioral aspects regarding approach and avoidance. However, only little is known about the situational underpinnings of these mechanisms, especially in terms of social influence. In three eye-tracking experiments, we tested whether attentional bias towards attractive faces is sensitive to situations involving social approach or social threat. We presented either appealing (social approach) or threatening (social threat) images, each followed by real world scenes depicting a more and a less attractive face. Results of the first experiment $(\mathrm{N}=44)$ show an interaction between attractiveness, condition, and subject's sex. For female participants, attractive faces were looked at longer in the social approach condition compared to the threat condition. For male participants, the opposite pattern was found. Results indicate that the situation evokes different functional aspects of facial attractiveness for male opposed to female respondents, potentially involving evolutionary adaptations in sexual selection and fight or flight. 


\title{
Visual influences
}

\section{Investigating Screen Center Bias and Orbital Reserve as Causes for Central Fixation Bias}

\author{
Lasse Borgholt, Peter Simonsen, Sigrid Klerke \\ University of Copenhagen, Denmark \\ lasseborgholt@adm.ku.dk
}

It has been established that human observers have a strong bias towards fixating on the center of image stimuli - a phenomenon known as central fixation bias (e.g. Tatler, 2007). Previous research suggests that several factors contribute to central fixation bias (Tseng et al., 2009). We examine two oft-conflated causes, namely screen center bias and orbital reserve. Whereas screen center bias refers to a tendency to fixate towards the center of the screen, orbital reserve denotes a bias towards the center of the eye's orbit (i.e. looking straight ahead). We presented 12 participants with 2x50 natural scene images displayed either at the center or the periphery of the screen while fixating the head position either directly facing the screen or slightly rotated. This allows us to evaluate the individual contributions of screen center bias and orbital reserve to central fixation bias. Our results show that screen center bias occurs on the initial fixation, with a significantly skewed distribution (57 \% of first fixations fell in the image quadrant closest to the screen center). However, as found with text-based stimuli (Vitu et al., 2004) the proposed bias towards the orbital center did not have any discernable effect on viewing behaviour.

\section{Comparison of models of visual attention in degraded images Michael Schneider ${ }^{1}$, Kun Guo ${ }^{2}$, Florian Röhrbein ${ }^{1}$ \\ 1 Technische Universität München \\ 2 University of Lincoln \\ m.schneider@tum.de}

For this contribution we investigated the impact of different noise types and intensities on human gaze behavior and on performance of state-of-the-art models of visual attention. The noise was chosen to be comparable to naturalistic visual impairments. Our results show that noise consistently influences duration and frequency of fixations on images. Noisier images receive less fixations, fixations last longer and are distributed closer around the center of the image, whereas the average number of fixations per second is only slightly reduced. These effects are mediated mainly by noise intensity. Difficulties at the level of visual and cognitive processing may have contributed to the effect by inhibiting saccade initiation. Comparing four high performing models of visual attention, we found that model performance was dependent on the metric used, and whether center bias was considered. The shuffled ROC score metric punishes models that include a form of center bias, while other metrics show better performance for models incorporating center bias. The Judd model showed the best performance using standard metrics, while the Adaptive Whitening Saliency model is best if we correct for center bias effects. Nevertheless, for our dataset, model performance was still considerably worse than human performance measured as inter-observer variance. 
Posters

\author{
Midas look: Fixation maps on virtual 3-D objects \\ Martin Christoph Cölln ${ }^{1}$, Kerstin Kusch ${ }^{2}$ \\ 1 Independent Researcher, Germany \\ 2 Technische Universität Dresden, Germany \\ m.coelln@gmail.com
}

Fixation maps (Wooding, 2002) provide a straight-forward and holistic representation of a viewers distribution of attention within a trial and scene. However, the creation of these maps requires stimuli to be static and two-dimensional. In dynamic visual environments positions of moving objects are volatile and thus gaze-to-object-mapping becomes nontrivial.

We introduce a novel method along with a ready-to-use free software implementation designed to overcome these restrictions. The presented tool allows to create and record experiments containing virtual 3-D scenes with animation, movement and user interaction while recording eye movements. Post-hoc, the registered data (3-D objects, interaction, and gaze positions) may be combined in a way, that accumulates fixation maps directly on the surface of 3-D objects. Effectively, viewers "paint" the virtual objects using their eyes.

The result of the calculation is a set of fixation maps that represent the viewers "attentional landscape" related to the 3-D scene content. The maps may be exported and further processed. The method is presented in context of a choice-based eye-tracking experiment. Subjects were asked to choose between different cups of yogurt, which were presented as 3-D objects and could be navigated, zoomed and rotated in a very natural way.

\title{
The impact of image size on eye movement parameters
}

\author{
Ricardo Ramos Gameiro ${ }^{1}$, Kai Kaspar ${ }^{1,2}$, Sontje Nordholt ${ }^{1}$, Peter König ${ }^{1,3}$ \\ 1 Institute of Cognitive Science, University of Osnabrück, Germany \\ 2 Social and Media Psychology, Department of Psychology, University of Cologne, Germany \\ ${ }^{3}$ Department of Neurophysiology and Pathophysiology, University Medical Center Hamburg Eppendorf, \\ Germany \\ rramosga@uni-osnabrueck.de
}

Under natural conditions humans enjoy a large field of view, yet for technical reasons eyetracking studies are mostly performed with much restricted visual displays. Here we investigated the effect of different image sizes on key eye movement parameters. Specifically, we asked whether viewing behavior scales with images size or is constant in absolute measures. Participants freely observed images of different category (webpages, urban scenes, nature scenes) varying in image size $(7,10,15,21,30 \mathrm{inch})$ while their eye movements were tracked. Results revealed a central spatial bias of fixations that increased linearly with stimulus size. Additionally, up to the largest stimuli used mean saccadic amplitudes also showed a linear increase. These results are compatible with a linear scaling of scanning behavior. Surprisingly, however, analyses showed that larger images led to a higher amount of fixations combined with a decrease of the mean fixation duration. Hereby, both parameters followed a logarithmic rather than linear trend.

Consequently, the size in which visual stimuli are presented significantly affects those parameters that are commonly investigated in many studies on overt attention. 


\title{
The role of surprisal in predicting gaze during a contour change detection task
}

\author{
Filipe Cristino, E. Charles Leek \\ School of Psychology, Bangor University, United Kingdom \\ f.cristino@bangor.ac.uk
}

The concept of 'surprisal' has been used to quantify shape information content (Shannon, 1948). Feldman \& Singh (2005) have shown that surprisal is greatest at regions of high negative (concave) curvature. We aimed to examine whether surprisal predicts gaze patterns and behavioural performance in a contour change detection task. Formally, surprisal comprises two components: magnitude of curvature (turning angle) and polarity (differential weighting of negative and positive curvature). Contour shapes were created using 16 random length wheel spokes, uniformly distributed and joined using cubic splines. We de-correlated regions of high surprisal with negative (concave) and positive (convex) curvature change by manipulating the magnitude of curvature. This gave rise to regions of high surprisal on both concave and convex parts. We found that RTs are better predicted by curvature polarity (concave/convex) than by surprisal. Eye movements during the learning phase are also better predicted by curvature polarity than by surprisal (magnitude of curvature change). This provides new evidence that shape perception is differentially sensitive to curvature polarity and the magnitude of curvature change. We hypothesise that curvature polarity is computed as a categorical feature, and that the perceptual system is relatively insensitive to metric variation in curvature magnitude.

\section{Prediction errors are modulated by inferred information from the blind spot}

\author{
Benedikt Valerian Ehinger ${ }^{1}$, Peter König ${ }^{1,2}$, Ossandón José ${ }^{1}$ \\ 1 Osnabrück University, Germany \\ 2 Department of Neurophysiology and Pathophysiology, University Medical Center Hamburg-Eppendorf, \\ Hamburg, Germany \\ behinger@uos.de
}

The probabilistic view of brain function postulates that the brain operates by testing and refining predictions about the world. Here, we tested the existence of sensory predictive signals and focused on vision, where eye-movements produce stimuli shifts that could, in principle, be predicted. We compared the electroencephalographic activity of responses after an eye movement to a peripheral visual stimulus, presented monocularly either inside or outside the blind spot. In some trials we exchanged the stimulus during the saccade. We analyzed the data using univariate general linear models, corrected for multiple comparison using threshold-free cluster based methods. We observe early $(<250 \mathrm{~ms})$ lateralized EEG responses and late $(>250 \mathrm{~ms})$ responses to saccade-contingent stimulus changes that suggest the occurrence of error signals to low and high level predictions within the visual modality. Remarkably, the late, change-related response was diminished when the change occurred in relation to a filled-in percept inside the subjects' blind spots. These results indicate that the predictions occur across multiple levels of visual processing and are based on generative models that can differentiate between bottom-up input originating from the outside world or inferred from surrounding information. 
Posters

\author{
Computational Eye Movements \\ Joo-Hwee Lim \\ Institute for Infocomm Research, Singapore \\ joohwee@i2r.a-star.edu.sg
}

Computer-based scene understanding systems process image sequence frame by frame, and pixel by pixel within each frame, aiming to aggregate pixels into coherent regions (e.g. segmentation) for meaningful interpretation (e.g. object recognition). Is this exhaustive approach a good way for solving ill-posed visual perception and cognition problems? Human visual systems are driven by visual attention whereby eye movements facilitate the selection of relevant areas in scene image to focus (by fovea) and process, while keeping a broad picture with summary statistics in peripheral visual field. Why can't we develop a saccadebased visual information processing approach which is both more natural and efficient?

Although bottom-up saliency-based attention helps to anchor visual fixation and has been an active area of research for many years, more often than not, task-based top-down visual attention plays more important role in directing our visual computational resources to accomplish our activities. We have developed a new computational visual attention model and collected many hours of eye tracking data of subjects performing daily tasks (e.g. walking, reading, social interaction etc). We show that how top-down contextual guidance dominates over bottom-up cues in analyzing scenes, echoing meaningful saccades and fixations in the data.

\title{
How depth influences eye movements when viewing stereoscopic images of natural scenes \\ Christophe Maggia ${ }^{1}$, Anne Guérin-Dugué ${ }^{2}$, Nathalie Guyader ${ }^{2}$ \\ ${ }^{1} \mathrm{GIN}$, France \\ 2 GIPSA-lab, France \\ magg_chris@hotmail.fr
}

Contrary to the huge development of 3D devices, few eye-tracking studies have examined visual factors that guide explorations of 3D stimuli, either with artificial (Wang et al., 2012) or specific natural scene stimuli (Jansen et al., 2009). Authors agree with the existence of a depth bias making more and earlier eye fixations for areas closer to observer. However, how depth, monocular and binocular cues, modifies visual attention is still an open question. In this research, we built an image database containing scenes with perspective (geometrical or non-geometrical perspective) and scenes without any perspective. Scenes were displayed either in $2 \mathrm{D}$ or $3 \mathrm{D}$ viewing condition. The effect of the viewing condition, $2 \mathrm{D}$ vs. 3D, for these three scene categories, was analyzed, on various eye movement parameters across the exploration. For the $3 \mathrm{D}$ condition, fixations are shorter than for the $2 \mathrm{D}$ condition. The variability between the fixation positions of observers is larger for $3 \mathrm{D}$ than $2 \mathrm{D}$ condition. A depth bias is also observed in $3 \mathrm{D}$, as expected, but more interestingly, the bias also exists in $2 \mathrm{D}$ condition, highlighting the importance of the monocular depth cues. Finally, the vanishing point is the most attractive point, being gaze at first even before the closest object. 


\title{
The Useful Field of View in real world scene viewing: Tunnel vision versus general interference
}

\author{
Lester C. Loschky ${ }^{1}$, Ryan C. Ringer ${ }^{1}$, Zachary W. Throneburg ${ }^{2}$, Arthur F. Kramer ${ }^{3}$, \\ Aaron P. Johnson ${ }^{4}$ \\ ${ }^{1}$ Dept. of Psychological Sciences, Kansas State University, United States of America \\ 2 Dept. of Computing \& Information Sciences, Kansas State University, United States of America \\ ${ }^{3}$ Beckman Institute, University of Illinois at Urbana-Champaign United States of America \\ ${ }^{4}$ Department of Psychology, Concordia University, Canada \\ loschky@ksu.edu
}

In theories of visual attention, an important construct is attentional breadth-the spread of attention across the visual field-also called the "useful field of view (UFOV)," or the "perceptual span." An associated construct is "tunnel vision," whereby under an attentional load, the UFOV decreases as retinal eccentricity increases. However, visual performance also decreases due to eccentricity-dependent, acuity limitations, which most UFOV measures do not compensate for. This may explain why different studies of the effects of attentional load on the UFOV have found evidence sometimes for tunnel vision and other times for "general interference"-a uniform decrement across eccentricities. We dynamically measured the UFOV using a Gabor patch orientation discrimination task presented during natural scene viewing. We presented Gabors gaze-contingently on $14 \%$ of fixations, masked them to limit processing time, and size-scaled them at each eccentricity to factor out eccentricity-dependent acuity limitations. In two experiments using a dual-task paradigm, we varied attentional load using an auditory N-back task (Exp 1), and a gaze-contingent, foveal, rotated-L-versus-T discrimination task (Exp 2), which produced evidence of general interference, and tunnel vision, respectively. Thus, a simultaneous foveal load is necessary to produce tunnel vision in natural scene viewing. [Supported by the Office of Naval Research (\#10846128).]

\section{Pilots' Saccadic Eye Movements and Attention Shift during Flight Operations}

\author{
Wen-Chin $\mathrm{Li}^{1}$, Chung-San $\mathrm{Yu}^{2}$, Matthew Greaves ${ }^{1}$, Graham Braithwaite ${ }^{1}$ \\ ${ }^{1}$ Safety and Accident Investigation Centre, Cranfield University, Bedfordshire, UK \\ ${ }^{2}$ Department of Industrial Engineering and Engineering Management, National Tsing Hua University, \\ Hsinchu, R.O.C. \\ wenchin.li@cranfield.ac.uk
}

Saccadic eye movements are closely linked to attention distribution. A total of thirty-seven qualified mission-ready F-16 pilots participated in this research. The participants' flying experiences varied between 372 and 3,200 hours $(M=1280, S D=769)$. The eye movement data in this study are collected by three phases of visual behaviours during tactical operations: searching for the target with eye contact (Searching), pursuing the target for aiming (Aiming), and lock-on the target for pick-off (Lock-on). The results showed there were significant differences at pilots' saccade duration among three operational phases, $\mathrm{F}(2,108)=29.06$, $\mathrm{p}<.001, \eta_{p}^{2}=.447$. Further comparisons by post-hoc Bonferroni adjusted tests showed that saccade duration at the searching phase was significantly longer than aiming and lock-on, however, there was no significant difference at pilots' saccade duration between aiming and lock-on phases. Also, there were significant differences at saccade velocity among three operational phases, $\mathrm{F}(2,108)=7.87, \mathrm{p}<.005, \eta_{p}^{2}=.179$. Current research indicates that saccadic eye movement related to attention across different operational phases to allow pilots prioritized localization, identification and pursuing target. The findings could be further developed for advanced simulator training syllabi to improve pilots' situational awareness for tactical operations. 
Posters

\section{Normalized eye movement metrics across motor simulation states: a difference of perspective? \\ Sheree Ann McCormick, Nicholas Costen, Paul Holmes \\ Manchester Metropolitan University, United Kingdom \\ s.mccormick@mmu.ac.uk}

Introduction:

Eye movement metric congruency across motor simulation states is appealing for proponents of shared representation models; data supporting this contention are, however, conflicting. This study used a novel method for normalizing and analyzing gaze metrics to compare eye movements during action observation (AO) and motor imagery (MI) from allocentric and egocentric perspectives.

Method:

Spatial and temporal fixation data were collected as participants observed and imagined upper limb movements from two visual perspectives. The data in the four conditions were normalized for scale and orientation and segmented into three fixation point centers.

Results:

There were significant differences in the distribution of the means of the fixation point centers between $\mathrm{AO}$ and $\mathrm{MI}$ in the allocentric but not the egocentric perspective. Differences were also observed in the covariance of fixation-points within fixation centers between $\mathrm{AO}$ and $\mathrm{MI}$ between the two perspectives. There were also significant interactions for fixation duration and number of fixations in the two perspectives.

Discussion:

Eye movements across $\mathrm{AO}$ and $\mathrm{MI}$ conditions are more consistent from an egocentric perspective but information processing demand, irrespective of perspective, is reduced in MI. Differences may be due to the greater control of goal outcome in the $\mathrm{AO}$, egocentric condition. 


\title{
Visual search
}

\section{Pupil dilation indicates mind-wandering in visual search depending on task difficulty}

\author{
Christoph Huber-Huber, Ulrich Ansorge \\ University of Vienna, Austria \\ christoph.huber-huber@univie.ac.at
}

Mind-wandering is a neglected factor in visual search where theories focus on the role of display-directed attention alone (Bundesen, Habekost, Kyllingsbaek, 2005). However, work on mind-wandering and task-unrelated thoughts (Schooler \& Smallwood, 2006) suggests that attention is not always directed to the visual field. By inserting occasional thoughtprobes in a visual search experiment, we determined the relation between mind-wandering and performance. Additionally, by means of eye-tracking we investigated whether pupil size could serve as a marker of mind-wandering. Our results indicate that mind-wandering is associated with longer response times and more errors. In large but not in small set size conditions, pupillary responses evoked by the stimulus display were more pronounced when participants did not report mind-wandering. Given that pupil dilation indicates cognitive effort (Kahneman, 1973), these results point towards a link between search difficulty and mind-wandering suggesting that easy search tasks can be performed even when mindwandering, whereas the higher difficulty of the large set size condition is only reflected in task-evoked pupillary responses when attention is directed to the search display.

\section{Searching two hemifield-scenes simultaneously with the contralateral left and right hemisphere}

\author{
Richard Shillcock, Jiayi Cheng, Hadi Mehrpouya \\ The University of Edinburgh, United Kingdom \\ rcs@inf.ed.ac.uk
}

We describe an experiment employing a new visual search task: two different scenes are searched simultaneously as separate hemifields by the relevant hemispheres, under monocular presentation. The initially mid-screen fixation point defines the location of the vertical division between the two hemifields, revealing only half of each scene. Each scene can be explored by an eye-movement, only at the expense of the other scene. The task was to locate/fixate a person in one of two street scenes, a task the human visual system prioritizes (New, Cosmoses, \& Tooby, 2007), or to conclude that neither current scene contains a person. Participants robustly preferred the first saccade to be to the right of the initial, central fixation point. We interpret this behaviour as the strategic use of the right hemisphere's attentional bias towards panoramic processing (cf. Nuthmann \& Matthias, 2014). Participants flexibly created a pseudo-fovea to better inspect a particular target. We describe the effects on eye-movements when a previously unseen target is revealed by an eye-movement. We discuss the potential of this new experimental paradigm in terms of exploring hemispheric differences in search and attention. 
Posters

\title{
Find the fork in the bedroom! Contextual cueing for semantic intruders
}

\author{
Sabine Öhlschläger, Melissa Le-Hoa Võ \\ Goethe University Frankfurt, Germany \\ oehlschlaeger@psych.uni-frankfurt.de
}

Contextual cueing has shown to be stronger in meaningful than random contexts. Is this true when the search target itself is defined only by semantics? Participants repeatedly searched through displays consisting of object thumbnails to find the one object that did not match the scene category implied by the distractors (semantic intruder). The scene category display either did or did not predict the intruder position. Moreover, we manipulated the meaningfulness of displays: For half of the participants the distractors were ordered in a syntactic way (pot above stove), whereas for the other half, the objects were identical but randomly displayed. No advantage for predictive over non-predictive scene categories was observed. Responses tended to be faster, but more error-prone in the syntactic than the random condition. Presenting a semantic scene category word-prime immediately before search display onset not only reduced overall error rates and reaction times, but also time to first target fixation, implying that not only the decision process, but also the guidance to the semantic intruder was made more efficient. Altogether, these results suggest that searching for a semantic intruder can be aided more by the availability of scene category information than learning the contingencies of the intruder location.

\section{Target detection in dynamically changing visual displays: Eye movements suggest two modes of search}

\author{
Alex Muhl-Richardson ${ }^{1}$, Hayward J. Godwin ${ }^{1}$, Matthew Garner ${ }^{1,2}$, Julie A. Hadwin ${ }^{1}$, \\ Simon P. Liversedge ${ }^{1}$, Nick Donnelly ${ }^{1}$ \\ 1 School of Psychology, University of Southampton, UK \\ 2 Clinical and Experimental Sciences, Faculty of Medicine, University of Southampton, UK \\ alex.muhl-richardson@soton.ac.uk
}

Many real-world tasks involve monitoring complex displays of dynamically changing visual indicators, e.g. warning lights turning red. Target detection in these tasks may be driven by responses to specific target onsets or monitoring iterative changes to potential targets over time (search mode). Typically, search tasks use static displays, but in this study, participants completed a novel dynamic search task. Participants searched arrays of squares that changed between 16 colours at varying rates and responded via mouse-clicks on specific colour targets. Target prevalence (proportion of target-present trials) was manipulated within-participants at low (6\%) and high (66\%) levels.

Eye movement data revealed that $46 \%$ of targets were detected via responses to specific target onsets, but that others were detected predictively through monitoring of likely targets. There was also an effect of target prevalence upon response times, such that detection was slower at low prevalence, which was driven by extended verification times. In summary, the prevalence effects found here bear similarities to standard search, but act via slowed target verification, and there is evidence for two separable modes of search in dynamically changing displays. Additional analyses will examine patterns of eye movements associated with target monitoring in relation to prevalence and search mode. 


\title{
Worth a look: The effects of irrelevant rewarding stimuli on eye movements
}

\author{
Sage E. P. Boettcher, Melissa Le-Hoa Võ \\ Goethe University Frankfurt, Germany \\ sage.boettcher@gmail.com
}

It is known that value can dictate our behavior. This effect has been shown in pop-out search tasks where previously high-value targets attracted more saccades than their lowvalue counterparts (Theeuwes \& Belopolsky 2012). However, it is not yet established if rewarding stimuli also hold attention longer. In the current study, observers searched through visual displays containing randomly dispersed thumbnails of objects over two blocks.

During the training block, observers received varying amounts of reward (low vs. high) for two target types. There was a significant interaction of dwell time on the target over block half and target value. That is, observers needed less time to decide a high value stimulus was a target relative to a low value target as the block progressed.

In the test block, observers searched through displays for other targets. Items from the training block's target categories now appeared as critical distractors in the test block. Crucially, when the critical distractor was a high-value object response times increased relative to the low-value distractors. Additionally, dwell times on high-value distractors were longer relative to the low-value distractors, indicating an inability to disengage from previously rewarding stimuli thus holding attention longer.

\section{What eye movements can reveal about the coupling of attention and context memory in visual search}

\author{
Efsun Annac ${ }^{1}$, Mathias Pointner ${ }^{1}$, Hermann J. Müller ${ }^{1,2}$, Thomas Geyer ${ }^{1}$ \\ ${ }^{1}$ Ludwig Maximilians University Munich, Germany \\ 2 Birkbeck College, University of London, England \\ Efsun.Annac@psy.Imu.de
}

In everyday scenes, searched-for targets do not appear in isolation, but are embedded within configurations of non-target or distractor items. If the position of the target relative to the distractors is invariant, such spatial contingencies are implicitly learned and come to guide visual scanning ("contextual cueing" effect). Here we investigated the coupling between attention and implicit context memory using eyetracking. Observers performed a visual search task with half of the trials containing repeated displays. The search was followed by a recognition task. Observers had to judge the likely quadrant of the target in a former search display (with the target in the recognition display being replaced by an additional distractor stimulus). In Experiment 1, observers were allowed to move their eyes in each trial. It was found that the target quadrant was fixated longer in correct relative to incorrect trials (hit vs. false alarm responses, respectively). In Experiment 2, observers were cued to fixate and judge individual display quadrants. Recognition accuracy was higher when the two quadrants were congruent. We conclude that (1) eye movements are a reliable measure for implicit context memory in visual search; and (2) eye movements serve a functional role for retrieval from implicit context memory. 
Posters

\title{
Central and peripheral scene degradation modulate the detectability of peripheral targets
}

\author{
Anke Cajar, Paul Schneeweiß, Jochen Laubrock, Ralf Engbert \\ University of Potsdam, Germany \\ cajar@uni-potsdam.de
}

Degrading scenes in the central or the peripheral visual field by gaze-contingent filtering yields a characteristic pattern of mean saccade amplitudes: Central degradation provokes longer saccades and peripheral degradation provokes shorter saccades than an unfiltered control. Assuming that saccade amplitudes are related to the area of attentional selection, these effects suggest more focused attention in the central region with peripheral filtering and an attentional bias toward the periphery with central filtering. To test this hypothesis, we investigated in an eye-tracking experiment if the detectability of a peripheral target changes during scene viewing when low or high spatial frequencies are filtered in the central or the peripheral visual field. Viewers were required to detect green circles occurring in the periphery during critical fixations. Results confirmed the characteristic pattern of mean saccade amplitudes. Compared with an unfiltered control, target detectability decreased with peripheral filtering. We conclude that attention shrinks with peripheral filtering, thus producing tunnel vision with lower visual sensitivity to peripheral information. With central filtering, target detectability was unaffected. Therefore, increasing foveal processing difficulty does not impair the detectability of peripheral objects. Our results suggest that attentional detectability and saccadic selection are operating via overlapping, but not identical pathways.

\section{The relationship of long-term and short-term perceptual hypotheses - Evidence from contextual cueing of interrupted visual search}

\author{
Bernhard Schlagbauer ${ }^{1,2}$, Maurice Mink ${ }^{1}$, Hermann J. Müller ${ }^{1,3}$, Thomas Geyer ${ }^{1}$ \\ 1 Department Psychologie, LMU München, Germany \\ 2 Graduate School of Systemic Neurosciences, LMU München, Germany \\ 3 School of Psychology, Birkbeck College, University of London, UK \\ bernhard.schlagbauer@psy.Imu.de
}

In visual search, repeating the arrangement of search items leads to performance benefits, an effect termed contextual cueing. Another example of memory-based search is rapid resumption of interrupted search, which refers to the ability of observers to quickly resume visual search in a repeatedly presented display (for e.g., 100ms) interrupted by blank screens (for e.g., $900 \mathrm{~ms}$ ). Both rapid resumption and contextual cueing can be considered as instances of a predictive coding mechanism, forecasting the likely location of the target, which raises the question whether they are driven by a mutual process.

The present study investigated whether contextual cueing in an interrupted search task influences the rapid resumption effect. We could show that predictions formed in long-term memory did not interact with predictions in short-term memory (i.e. rapid resumption), while contextual cueing still facilitated overall performance. Specifically, the analysis of eye movements revealed that fast responses to the target were not affected by repeated context, but the distance to the target during the first brief presentation of the displays was reduced. We propose that this is achieved by means of reducing the amount of items that have to be considered as likely targets, resulting in a head-start of the search process. 


\title{
Visual search vs. visual foraging in studying visual attention
}

\author{
Ómar I. Jóhannesson ${ }^{1}$, Ian M. Thornton ${ }^{2}$, Irene J. Smith ${ }^{1}$, Andrey Chetverikov ${ }^{3,4}$, Árni \\ Kristjánsson ${ }^{1,5}$ \\ ${ }^{1}$ University of Iceland, Reykjavík, Iceland \\ ${ }^{2}$ University of Malta, Msida, Malta \\ ${ }^{3}$ Saint Petersburg State University, Saint Petersburg, Russia \\ ${ }^{4}$ Russian Academy of National Economy and Public Administration, Moscow, Russia \\ ${ }^{5}$ University College London, London, UK \\ oij1@hi.is
}

Visual search with and without eye movement tracking, has shed important light upon the function of visual attention. We introduce a new foraging paradigm that may be more realistic for our day-to-day tasks. Animals prefer to forage on the same type of prey in long runs when it is hard to discriminate between prey types and in short runs when the discrimination is easy (Dukas \& Ellner, 1993). Humans show similar two-step pattern when foraging with their fingers (Kristjánsson, Jóhannesson, \& Thornton, 2014). Before a target can be selected it has to be found, which depends on visual attention and eye movements. This may suggest a close relationship between finger and gaze foraging. We present results from a novel foraging task where observers "pick" the targets by fixating them. The run length for the majority of the participants during eye tracking was similar when the target was defined by one feature and by a conjunction of two features. The same observers showed the twostep, animal like pattern when foraging by finger taps. This suggests that it may be easier to switch between attentional templates when only the eyes rather than fingers are required for selection.

\section{On the (un)importance of foveal vision during visual search in real-world scenes}

\author{
Adam C. Clayden, Robert B. Fisher, Antje Nuthmann \\ University of Edinburgh, United Kingdom \\ s1475487@sms.ed.ac.uk
}

The importance of foveal vision to a visual-cognitive task can be assessed by denying foveal vision using the gaze-contingent Moving Mask technique. Foveal vision was found to be necessary when searching for a target letter in alphanumeric displays (Bertera \& Rayner, 2000). In contrast, foveal vision was not necessary to correctly locate and identify mediumsized target objects in natural scenes (Nuthmann, 2014). The present study combines design features from both paradigms such that observers searched for the letter " $\mathrm{T}$ " embedded in greyscale pictures of real-world scenes. To control for visual salience, the letter was algorithmically placed for each scene in a location for which there was a medium change in local contrast when inserting the letter. Four letter sizes, ranging from $0.25^{\circ}$ to $1.5^{\circ}$ in width, were crossed with the presence vs. absence of foveal vision. Search times increased as target size decreased. When searching the scene with artificially impaired foveal vision, search performance was largely unimpaired, in correspondence with Nuthmann (2014). Only when searching for the smallest letter, target verification time but not scanning time was elevated. Foveal vision was more important in a second experiment, which asked observers to decide whether the target was a $\mathrm{T}$ or $\mathrm{L}$. 
Posters

\title{
Competition for perceptual salience in eye guidance during realistic scene search
}

\author{
Sara Spotorno ${ }^{1}$, Benjamin W. Tatler ${ }^{2}$ \\ 1 University of Aberdeen, United Kingdom \\ 2 University of Dundee, United Kingdom \\ sara.spotorno@abdn.ac.uk
}

Low-level eye guidance during search remains unclear. Using realistic scenes containing a target and a distractor, we examined the impact of the perceptual salience of these two objects, measured with state-of-art computational algorithms. We also analysed salience interaction with high-level guidance supplied by the type of target template (verbal vs. pictorial) and expectations about object placements (consistent vs. inconsistent). A pictorial template enhanced all search phases, independently of object salience. High target salience improved accuracy of search initiation significantly only for targets in inconsistent placements. The presence of a highly salient object reduced first saccade latency, and the fastest initiation was found when the target was more salient than the distractor. These results suggest that the visual system computes salience of competing objects early during scene viewing, and utilises it flexibly as a powerful source of object selection. Scene scanning was fast and affected by target salience, mainly in inconsistent spatial conditions, but not by distractor salience. When the distractor occupied a target expected location, and the target was inconsistently placed, high distractor salience shortened target foveal inspection, suggesting that early distractor selection according to its low-level features may reduce its later interference on verification processes.

\section{Let's inhibit anyway! Inhibition of saccadic return for search-relevant and search-irrelevant items Margit Höfler ${ }^{1}$, lain D. Gilchrist ${ }^{2}$, Christof Körner $^{1}$ \\ 1 University of Graz, Austria, Department of Psychology \\ 2 University of Bristol, UK, School of Experimental Psychology ma.hoefler@uni-graz.at}

When participants search the same display consecutively for two different targets, inhibition of return (IOR) operates in both searches. Here we tested whether IOR in such repeated search is limited to those items in the first search that are not relevant for the second search. In the task, participants searched a display twice which consisted of letters of two colours. In the first search, the target colour could be either pink or blue. In the second search, the target was always of one colour. Thus, only half of the items in the first search were relevant for the second search because they could become the future target. In order to measure IOR in the first search, a probe was presented at an item that was either relevant or not for the second search. The probed location was either previously inspected (old probe) or not (new probe). Participants were instructed to saccade to the probe and then to continue searching. Saccadic latencies to old probes were longer than to new probes, thus demonstrating IOR. However, this difference occurred regardless of item relevance. This suggests that IOR is operating during the first search irrespective of the future relevance of inspected items. 


\title{
Reward modulates oculomotor competition between distractors and target in top-down visual search
}

\author{
Cao $\mathrm{Ge}^{1}$, Ruimeng Wang ${ }^{2}$, Xin Zhao ${ }^{2}$, Yi Zhang ${ }^{2}$, Yang Wang ${ }^{2}$, Haibo Yang ${ }^{2}$ \\ 1 Zhengzhou University, People's Republic of China \\ 2 Tianjin Normal University, People's Republic of China \\ chaoge101@zzu.edu.cn
}

It has long been known that attention selects stimuli that are task relevant or perceptually salient. Recent findings indicate that rewards exert a powerful influence on the deployment of visual selective attention. This study examine whether the capture of attention by previously reward-associated stimuli is modulated by the processing of current but unrelated rewards. Thirty-six teenagers learned to associate two color stimuli with different amounts of reward during a training phase. In a subsequent test phase, these previously rewarded color stimuli were occasionally presented as to-be-ignored distractors while participants performed visual search for each of two differentially rewarded color-defined targets. Eye movements were record with SMI RED500 eye tracker.The results reveal that attentional capture by formerly rewarded distractors was the largest when reward were the highest in the test phase, even though such rewards were unrelated to the color distractors. Reward associated objects affected saccades in visual search and high reward objects attracted the eyes more strongly than low and no reward objects. The results imply that reward affects oculomotor competition in favor of stimuli previously associated high reward when multiple reward associated objects compete for selection.

\section{Dominant eye vs. Cyclopean eye in gaze interface tracking Michael Wagner, Tomer Elbaum, Assaf Botzer \\ Ariel University, Ariel Israel, Israel wag.michael@gmail.com}

Ocular dominance is defined as superiority of one eye over the other in perceptual motor tasks, such as object's locations. Unlike "ocular dominance theory", the "cyclopean eye theory" claims no role of ocular dominance in perceiving object's locations. We performed two smooth-pursuit target-tracking experiments, investigating the role of ocular dominance, measuring tracking accuracy:

In Experiment-1 participants performed a free visual target tracking task (open-loop).

In Experiment.-2 participants performed a gaze-interface target-tracking task (Feedbackcursor coupled to the dominant or the cyclopean eye). Tracking task was composed of straight or curved target-motion- lines (4 velocity levels).

"Ocular dominance theory" proposes the sighting-dominant eye as a reference point for visual direction. Accordingly, we expected in Experiment-1 higher dominant eye-to-target accuracy. In-contrast, "cyclopean eye-theory" proposes mid-binocular location as reference point for visual direction. Accordingly, higher cyclopean eye-to-target accuracy was expected.

In experiment- 2 the two theories' predictions were expected to match the eye-cursor coupling method.

Our results show higher tracking accuracy of the "Cyclopean eye" in experiment 1, and higher performance efficiency with binocular cursor coupling in experiment 2, indicating an advantage of the "Cyclopean eye theory". In turn, results contradict the proposed analogy of eye and hand dominance. Implication for gaze interface is presented. 
Posters

\section{Applying eye tracking technologies in foreign language vocabulary learning studies \\ Anna Izmalkova, Irina Blinnikova \\ Moscow State Linguistic University, Russian Federation mayoran@mail.ru}

Eye movement data was collected and analyzed from 31 subjects (in total 755 valid trials), while they were learning word pairs (native language (Russian) word - pseudoword (modeling a foreign word)). Quantitative recall data was counted, mnemonic techniques were distinguished and recall mistakes were analyzed. Three most frequently used mnemonic techniques were revealed: "graphical technique", "phonological technique" and "semantic technique". The techniques correspond with the levels of processing as proposed by F.Craik, R.Lockhart (1972) and B. Velichkovsky (1999).

Recall score was connected with the strategy used $F(755)=11,2(p<0,01)$, escalating with the increasing "depth" of processing. The strategies were reflected in eye movements: "graphical" strategy was associated with more fixations; "phonological" strategy was characterized by more switches between AOIs (native and quasi-foreign word) and less fixations on the AOIs; "semantic strategy" was distinguished by fewer switches between AOIs and longer fixation duration.

In addition, if first fixation was on the Russian word, less switches were made between AOIs, than if subjects looked at the quasi-foreign word first. In the first case the new word form could be included into the logogen (in terms of J.Morton) faster as the logogen was activated earlier. 


\section{Thursday, August $20^{\text {th }}, 3.00 \mathrm{pm}-5.00 \mathrm{pm}$}

\section{Attention capture/Oculomotor capture}

\section{Feature priming rather than visual working memory affects oculomotor selection in a bottom-up manner}

Mieke Donk, Jeroen Silvis, Jozua Murris, Artem Belopolsky

Vrije Universiteit Amsterdam, Netherlands, The

w.donk@vu.nl

Several studies demonstrated that objects held in working memory can influence early oculomotor selection. The goal of the present study was to investigate whether these effects are mediated by the active maintenance of features in visual working memory or by feature priming. Two experiments were performed in which participants were asked to saccade to a target line segment of a certain orientation that was presented together with a to-beignored distractor line segment of a different orientation. Target and distractor were given a task-irrelevant color that varied per trial. In a secondary task, a color had to be memorized, and that color could either match the color of the target, match the color of the distractor, or did not match the color of any of the objects in the oculomotor task. The memory task was completed either after the oculomotor task (Experiment 1), or before it (Experiment 2 ). The results showed that memory content biased oculomotor selection, especially for the short-latency saccades. Crucially, the time course of this effect was similar in both experiments suggesting that bottom-up feature priming rather than the active maintenance in visual working memory is the driving force behind early biases in oculomotor selection.

\section{Subliminal oculomotor capture: The role of contrast polarity Hanna Weichselbaum ${ }^{1}$, Isabella Fuchs ${ }^{2}$, Ulrich Ansorge ${ }^{2}$ \\ ${ }^{1}$ Cognitive Science Research Platform, University of Vienna \\ 2 Faculty of Psychology, University of Vienna hanna.weichselbaum@univie.ac.at}

Subliminal stimuli can capture attention according to the observer's goals. For instance, when looking for black targets, only subliminal black but not white distractors capture attention. In contrast, the bottom-up explanation claims that attention to subliminal stimuli is driven by the salience of the stimulus. When looking for black targets, all salient stimuli (e.g., black and white distractors) should capture attention. We tested these two assumptions by measuring subliminal oculomotor capture (Weichselbaum, Fuchs, \& Ansorge, Vision Research 100, 2014). Subliminal singleton-onset distractors were presented either in the same or the opposite vertical hemifield as the target. The distractor had either the same or not the same contrast polarity as the target. We measured saccade latency, saccade trajectory deviation, and saccade endpoint deviation. Oculomotor capture was shown by shorter saccade latency with distractors in same- as compared to opposite-hemifield targets. In line with a bottom-up explanation of subliminal oculomotor capture, this effect was independent of the similarity between the distractor's contrast polarity and that of the searched for target. 
Posters

\section{Perceptual saliency biases fixations on a surprise trial Gernot Horstmann \\ Bielefeld University, Germany \\ gernot.horstmann@uni-bielefeld.de}

Promient psychological and neuro-computational models predict that salience captures attention. This proposition, however, has recieved mixed results in experimental research and there are strong theoretical arguments against saliency capture. Most arguments do not apply for a surprise presentation. Previous studies have indeed shown that a surprise saliency attracts attention. In these studies, however, the salient feature was also always the only novel feature in the display. The present study presents a salient feature in a surprise trial where all stimuli have novel features. Results show that fixations are biased towards the novel feature. The effect is strong, but much later than expected on the basis of saliency models.

\section{Is oculomotor capture due to attentional capture or to saccade priming? \\ Tobias Schöberl, Ulrich Ansorge \\ University of Vienna, Austria \\ tobias.schoeberl@univie.ac.at}

Abrupt onset cues attract people's gaze. This oculomotor capture, is often attributed to automatic attraction of spatial attention, meaning that a valid cue at target position facilitates target representations and an invalid cue away from the target delays it. Alternatively, oculomotor capture could reflect an automatic activation of a saccade towards the onset cue, meaning that there is less conflict between cue- and target-elicited saccade activation in valid than invalid conditions.

Here, we addressed this question: Participants made speeded saccades towards a left or right black target (pro-saccade task) or away from it (anti-saccade task). Irrelevant white abrupt onset cues were unpredictably and equally likely valid or invalid. If these cues attracted attention, we expected a validity effect (faster target-directed saccades in valid than invalid conditions), both in pro- and anti-saccade tasks because a target would benefit from valid cues, regardless of the responses. In contrast, if abrupt onset cues directly influenced saccade programming, a validity effect was expected in the pro-saccade task, but no or a reversed validity effect was expected in the anti-saccade task, because in the anti-saccade task, valid cues would activate incorrect and invalid cues correct responses. Results supported the latter of these possibilities. 


\title{
Saccadic Eye Movements Reveal Stronger Attention Capture by Fearful than Neutral Faces
}

\author{
Shah Khalid ${ }^{1}$, Ulrich Ansorge ${ }^{2}$ \\ ${ }^{1}$ Institute of Cognitive Science, University of Osnabrück, Germany \\ ${ }^{2}$ Faculty of Psychology, University of Vienna, Austria \\ shah.khalid@uni-osnabrueck.de
}

Immediate threat might capture attention automatically, a phenomenon called threat advantage. Using saccadic eye movements we tested capture by fearful faces in an exogenous cuing experiment. The different face images were carefully equated for their spectral power and their contrast. Per each trial, a pair of faces, one neutral and one fearful was presented as a task-irrelevant cue. It was shown prior to the target with a cue-target SOA of $20 \mathrm{~ms}$ or $250 \mathrm{~ms}$ for a duration of either $20 \mathrm{~ms}$ or of the full SOA. The fearful face was shown either at the target's position (valid threat condition) or away from the target (invalid threat condition) along with the neutral face on the opposite side. We expected a threat advantage, with better saccadic performance in the validly than invalidly threat-cued conditions. Our results confirmed a threat advantage. Performance was better in valid than invalid conditions. In addition, control conditions with inverted (i.e., upside-down) faces as cues confirmed that the threat advantage was face-specific and that it was only found with upright but not with inverted faces. Our results confirmed the rapid capture of attention by fearful faces. Results are discussed in light of existing theories.

\section{A Comparison of Bottom-up and Top-down Salience between Human Vision and a Computer Model of Visual Attention}

\author{
Simone Frintrop, Lina Jackel, Ulrich Ettinger \\ Rheinische Friedrich-Wilhelms-Universität Bonn, Germany \\ frintrop@iai.uni-bonn.de
}

We have compared human fixations with the data from a computational model of visual attention, to investigate the influence of bottom-up and top-down cues on human eye movements. Unlike other studies which usually use "bottom-up" saliency maps (e.g. Henderson et al, 2009), our computer model is able to generate "top-down" saliency maps that consider the properties of a target. This enables a direct comparison of visual search performance in which not only human subjects but also the computer model is able to exploit target knowledge.

Our results show that items that are considered salient by the computational system are fixated more frequently and are detected faster by humans in a visual search task. Here, not only the top-down salience but also the bottom-up salience of items facilitated the search for human observers. Interestingly, in a free-viewing task, the bottom-up salience of items had no statistically significant effect on the fixations. This might indicate that bottom-up salience is more important for guiding visual search than for guiding eye movements under free-viewing conditions, an aspect we will further investigate in the future. 
Posters

\section{Variational saliency maps for dynamic image sequences}

Aniello Raffaele Patrone ${ }^{1}$, Christian Valuch ${ }^{3}$, Ulrich Ansorge ${ }^{3}$, Otmar Scherzer ${ }^{1,2}$

${ }^{1}$ Computational Science Center, University of Vienna, Austria

2 Johan Radon Institute for Computational and Applied Math RICAM

3 Department of Basic Psychological Research and Research Methods, University of Vienna, Austria aniello.patrone@univie.ac.at

Saliency maps are an important tool for modeling and predicting human eye movements but their applications to dynamic image sequences, such as videos, are still limited. In this work we propose a variational approach for determining saliency maps in dynamic image sequences. The main idea is to merge information from static saliency maps computed on every single frame of a video (Itti \& Koch, 2001) with the optical flow (Horn \& Schunk, 1981; Weickert \& Schnörr, 2011) representing motion between successive video frames. Including motion information into saliency maps is not a novelty but our variational approach presents a new solution to the problem, which is also conceptually compatible with successive stages of visual processing in the human brain. We present the basic concept and compare our modeling results to classical methods of saliency and optical flow computation. In addition, we present preliminary eye tracking results from an experiment in which 24 participants viewed 80 real-world dynamic scenes. Our data suggest that our algorithm allows feasible and computationally cheap modeling of human attention in videos. 


\title{
Clinical applications
}

\section{Eye Movements Reveal links between Attentional Biases to Threat and Friendship Quality in 9-11-year-olds with Internalising and Externalising Personality Traits.}

\author{
Katerina Pavlou, Julie A. Hadwin, Valerie Benson \\ University of Southampton, United Kingdom \\ kp1c11@soton.ac.uk
}

We tested the idea that the association between temperamental risk and poor peer relationships could result from anxious and aggressive individuals' difficulty to inhibit threat. We measured the ability of a group of 9-11 year-old English schoolchildren to direct their eyes towards and identify a non-face target in the presence of central, parafoveal and peripheral face distractors of different emotions. Self-reports of anxiety, neuroticism, attentional control and friendship quality were also obtained. All participants showed a normal remote distractor effect. Additionally, results also showed that neuroticism was related to increased saccade latencies to the target in the presence of angry (but not neutral or happy) face distractors for all eccentricities, indicating a broadening of attention for threat in that group. In contrast, psychoticism was found to be associated with a higher proportion of eye movements directed towards the distractor faces (errors) for all types of face distractors, suggesting automatic capture by all face distractors. Poor self-reported friendship quality was also linked to increased directional errors, but this was restricted towards angry distractors. The eye movement data suggest that neuroticism is linked to impaired inhibition of threat and that selective attention to threat is important in understanding peer relationship difficulties.

\section{Presymptomatic changes in saccadic eye movement in hemiparkinsonian MPTP-treated monkeys}

\author{
Leonid Victorovich Tereshchenko, Victor N. Anisimov, Valeriy V. Shulgovskiy, Alexander V. \\ Latanov \\ Moscow State University, Russian Federation \\ Iter@mail.ru
}

The temporal dynamics of saccadic parameters was studied in two monkeys which executed the visio motor task at the development of MPTP-model of Parkinson's disease (PD) caused by administration of low doses of MPTP. Eye movements were recorded by searchcoil method $(500 \mathrm{~Hz}$, spatial accuracy 0.1 degree). State of the MPTP-syndrome was rated against behavioral scale which includes ranking of ten symptoms of Parkinson's model. We studied the horizontal, vertical and oblique visually-guided and corrective saccades provoked by stimuli onset in various points within 2-dimensional visual field in the Gap-StepOverlap paradigm. Latencies of saccades with low amplitude (towards the targets of 6.5 deg. eccentricity) increased by $20-44 \% \%$ as compared to the normal rates at the presymptomatic state of syndrome development. Latencies of saccades with intermediate amplitude (targets of 13 deg.) increased to a lesser extent - by 5-17\% in this case. The presaccadic intervals of corrective saccades increased by $11-15 \% \%$. Those changes are statistically confirmed at presymptomatic state of the MPTP-syndrome. Significant changes in the execution rate of corrective and multisaccadic trials, peak saccadic velocity were developed later with manifestation of MPTP-syndrome. The obtained data can be used in development of methods for early PD diagnosis. 
Posters

\title{
To exploit or explore? Using pupillometry to track norepinephrine activity in adults with ADHD symptoms
}

\author{
Rebecca Nicole Elisa, Benjamin A. Parris \\ Bournemouth University, United Kingdom \\ relisa@bournemouth.ac.uk
}

Recent work has confirmed that changes in pupil diameter directly relate to changes in locus-coeruleus norepinephrine (LC-NE) system activity (Gilzenrat, Nieuwenhuis, Jepma, \& Cohen, 2010; Rajkowski, Kubiak, \& Aston-Jones, 1993). The two modes of LC-NE firing (tonic and phasic) are represented by different pupillary responses and promote either task engagement and exploitation (LC phasic mode), or task disengagement in favour of exploration (LC tonic mode). Attention deficit hyperactivity disorder (ADHD), in particular the symptom of inattention, is thought to be related to dysfunctional norepinephrine modulation. The present research investigates differences in LC-NE activity between participants with ADHD symptoms, and healthy controls. Pupil diameter is tracked during completion of the diminishing utility task (Gilzenrat et al. 2010). This task gives participants the opportunity to disengage from a block of trials in order to explore a new block when the expected value of continuing with the current block is diminished. It is predicted that participants with ADHD symptoms will show a tendency towards larger baseline pupil diameters and smaller stimulus evoked dilations, both of which represent LC tonic mode. This favour towards exploration should be reflected behaviourally by disengagement from trial blocks. Preliminary analysis on initial data indicates support for hypotheses.

\section{Emotion recognition and attentional deployment in adolescent, psychiatric patients with affective dysregulation}

\author{
Anne Kathrin Schwenzfeier ${ }^{1}$, Benjamin Pniewski ${ }^{1}$, Marlies Pinnow ${ }^{2}$, Anna Ball ${ }^{1}$, Martin \\ Holtmann $^{1}$, Tanja Legenbauer ${ }^{1}$ \\ ${ }^{1}$ LWL-university hospital for child and adolescent psychiatry Hamm, Germany \\ 2 Ruhr-University Bochum, Germany \\ anne_kathrin.schwenzfeier@wkp-lwl.org
}

Affective dysregulation (AD) is characterized by a deficit in regulating one's emotions. Approximately $1 \%$ of the children and adolescents of the general population and up to $7 \%$ of psychiatric samples suffer from symptoms associated with AD. It is reasoned that a lack of proper emotion recognition and attentional deployment cause AD. This study investigated to what extent adolescent, psychiatric patients with AD differ in terms of their ability to understand emotions and their attentional deployment from a clinical and a healthy control group (Groups:AffectiveDysregulation, ClinicalControlGroup, HealthyControlGroup).

Patients and healthy controls were confronted with a standardized facial picture set representing the following emotions in different intensities: Happy, anger, disgust, fear, sadness and surprise. While classifying the pictures, the hit rate, reaction times and attentional deployment measured by an eye tracker were assessed.

116 participants took part in the present study (AD:n=46, CCG:n=40, HCG:n=30). Patients with $\mathrm{AD}$ demonstrated greater difficulty to label emotions correctly and needed more time time to classify the emotions of diverse intensities. The present results highlight a general deficit in emotion recognition among patients with $\mathrm{AD}$. The eye tracking data will elucidate to what extent patients with AD can be characterized by a differential attentional deployment. 


\author{
Inefficient facial scan paths in autism? \\ Masaharu Kato ${ }^{1}$, Kosuke Asada ${ }^{2}$, Shinichiro Kumagaya ${ }^{2}$, Satsuki Ayaya ${ }^{2}$ \\ ${ }^{1}$ NTT Communication Science Laboratories, Japan \\ 2 The University of Tokyo \\ piekomk@gmail.com
}

Autism spectrum disorder (ASD) is a developmental disorder and individuals with ASD (ASDs) often struggle to process facial information, but why this occurs is not well understood.

We hypothesized that poor facial information processing by ASDs was explained by inefficient collection of information from faces. Then, the scan paths of ASDs should be dissimilar, because the increase in similarity between individuals' scan paths indicates the development of efficient eye movements, which leads to efficient collection of facial information (Kato and Konishi, 2013).

Facial images were presented in both upright and inverted orientations, and scan paths were analyzed for typically developed individuals (TDs; $n=16)$ and ASDs $(n=16)$. For upright faces, the similarity in scan paths among TDs was higher than among ASDs, and also higher than the similarity between ASDs and TDs. This group difference disappeared for inverted faces. Our results are in line with the known fact that upright faces are more salient and recognizable than inverted faces for TDs, and further support our hypothesis. Interestingly, similarity in intra-personal scan paths was marginally higher for ASDs than for TDs, suggesting that ASDs tend to look at faces in an inflexible way, irrespective of the specific face. 
Posters

\section{Fixational saccades in Multiple Sclerosis disorders}

João P. M. Costa ${ }^{5}$, Marisa B. Ferreira ${ }^{5,3}$, Marta Parreira ${ }^{3,5}$, Olga Joana Miguel ${ }^{5,6}$, Inês Sousa $^{6}$, José Figueiredo ${ }^{3,4}$, João José Cerqueira ${ }^{1,2,5}$, Paulo A. Pereira ${ }^{3,5,6}$, António Filipe Macedo $^{5}$

1 Neurosciences Domain

Life and Health Sciences Research Institute, School of Health Sciences and ICVS/3B's Associate Laboratory, University of Minho, Braga, Portugal

2 Clinical Academic Centre (CCA), Hospital of Braga, Braga, Portugal.

3 TEM -Association all with the Multiple Sclerosis, Braga, Portugal.

${ }^{4}$ Department of Neurology, Private Hospital of Braga, Braga, Portugal.

${ }^{5}$ Vision Rehabilitation Lab.

Centre/Department of Physics and Optometry, University of Minho, Braga, Portugal

${ }^{6}$ Department of Mathematics and Applications, University of Minho, Braga, Portugal joaopmoraiscosta@gmail.com

Research question

With this study we wanted to investigate the frequency and characteristics of fixational saccades in patients with Multiple Sclerosis (MS).

Methods

We report here data from 15 MS patients (relapsing-remitting course, EDSS $\leq 3$ ) and 15 controls. Eye movements were monitored using a binocular infrared eyetracker running at $250 \mathrm{~Hz}$ (RED250, SMI Gmb Germany). The task was to fixate during 10s a randomly presented dot in 3 positions: centre, 15deg-right or 15deg-left off the center of the screen. Saccades were detected when: eye speed was $15 \mathrm{deg} / \mathrm{s}$ and/or acceleration was 3500deg/s2 with a minimum duration of $12 \mathrm{~ms}$.

Results and their interpretation

Fixational saccades showed different frequency, amplitude, and peak-velocity amongst positions tested. Frequency, amplitude and peak-velocity were increased and duration was reduced in the MS-group when compared with control. Collapsing data from the 3 positions the mean and standard deviation (SD) values for these variables were: $1.42 \mathrm{sac} / \mathrm{s}(\mathrm{SD}=1.3)$, $1.18 \mathrm{deg}(\mathrm{SD}=0.92), 150.81 \mathrm{deg} / \mathrm{s}(\mathrm{SD}=101.5), 24.05 \mathrm{~ms}(\mathrm{SD}=8.68)$ in the MS-group. For the control-group values were: $0.84 \mathrm{sac} / \mathrm{s}(\mathrm{SD}=0.85), 1.16 \mathrm{deg}(\mathrm{SD}=1.25), 121.60 \mathrm{deg} / \mathrm{s}(\mathrm{SD}=98.95)$ and $27.8 \mathrm{~ms}(\mathrm{SD}=18.51)$. These results show that the control of fixational saccades is altered at early stages of multiple sclerosis. 


\section{Investigating fatigue in Multiple Sclerosis using saccades}

Marisa B. Ferreira ${ }^{1,2}$, Marta Parreira ${ }^{2}$, Olga Joana Miguel ${ }^{3}$, Inês Sousa ${ }^{3}$, José Figueiredo ${ }^{4}$, Paulo A. Pereira ${ }^{2,3}$, João José Cerqueira ${ }^{5,6}$, António Filipe Macedo ${ }^{1}$

1 Vision Rehabilitation Lab. Centre/Department of Physics and Optometry, University of Minho, Braga, Portugal.

${ }^{2}$ All with the Multiple Sclerosis, Braga, Portugal.

${ }^{3}$ Department of Mathematics and Applications, University of Minho, Braga, Portugal

${ }^{4}$ Department of Neurology, Private Hospital of Braga, Braga, Portugal.

${ }^{5}$ Clinical Academic Centre (CCA), Hospital of Braga, Braga, Portugal.

${ }^{6}$ Neurosciences Domain

Life and Health Sciences Research Institute, School of Health Sciences and ICVS/3B's Associate

Laboratory, University of Minho, Braga, Portugal. marisaborgesferreira@gmail.com

Research question:

In this study we investigated if patients with Multiple Sclerosis(MS) reporting fatigue have altered saccade parameters.

Methods:

Forty patients diagnosed with MS with relapsing-remitting course,EDSS $\leq 3$, participated in this study. Exclusion criteria were: relapses, cognitive impairment, TBI, stroke, depression or difficulties to calibrate the eyetracker. Eye movements were monitored using a binocular eyetracker running at $250 \mathrm{~Hz}$ (RED250, SMI-Gmb Germany). Saccades were performed to $10 \mathrm{deg}$ to the left or to the right of fixation target using an endogenous paradigm. Depression was evaluated with the Beck Depression Inventory, fatigue with the Fatigue Severity Scale and the impact of the fatigue with Modified Fatigue Impact Scale. Variables analyzed were saccade: amplitude, peak-velocity, latency and accuracy - final eye position(FEP) and absolute error(ABSerror).

Results and interpretation:

In total $55 \%$ of the participants scored a level of fatigue outside the normal range and $33 \%$ reported a significant impact of fatigue in their daily living. Fatigue magnitude was correlated negatively with peak-velocity, $r=-0.4(\mathrm{p}=.009)$, amplitude, $\mathrm{r}=-0.5(\mathrm{p}=.005)$ and FEP, $\mathrm{r}=-0.5(\mathrm{p}=.005)$ and positively correlated with latency, $\mathrm{r}=0.4(\mathrm{p}=.022)$ and ABSerror, $\mathrm{r}=0.4(\mathrm{p}=.008)$. Impact of fatigue was correlated negatively with the amplitude, $\mathrm{r}=-$ $0.3(\mathrm{p}=.037)$ and FEP, $\mathrm{r}=-0.3(\mathrm{p}=.037)$. Our results indicate that saccade pathways might be affected in patients with MS reporting fatigue. 
Posters

\title{
Eye tracking and attentional bias for emotion stimuli with symptoms of earthquake related PTSD
}

\author{
Haibo Yang, Xin Zhao, Ruimeng Wang, Yang Wang, Yi Zhang \\ Tianjin Normal University, People's Republic of China \\ yanghaibo@mail.tjnu.edu.cn
}

\begin{abstract}
Attentional bias is an occurrence wherein a person focuses more of his attention toward a specific stimulus or a sensory cue. Often, this leads to a poor sense of judgment or an incomplete recollection of a certain event or memory. Although several studies have documented an attentional bias toward threat in posttraumatic stress disorder (PTSD), the nature of attentional bias has not been clearly delineated. The present study utilized eye tracking technology to delineate the time course and components of attentional bias for emotion pictures in earthquake related PTSD. Earthquake survivor teenagers with PTSD symptoms $(\mathrm{n}=21)$, earthquake survivor teenagers without PTSD $(n=20)$, and non-trauma teenagers control group $(n=20)$ viewed trauma related pictures paired with neutral pictures for 3 s presentations. Eye movements were record with SMI RED500 eye tracker. The results showed that teenagers with PTSD maintained attention longer on the emotion pictures. This attentional bias was robustly distinguished teenagers with PTSD from both teenagers without PTSD and non-trauma teenagers controls. Its indicated that dwelling on emotion cues may reflect current PTSD symptoms, and it could reflect a cognitive vulnerability factor for PTSD.
\end{abstract}

\section{Eye movement based evaluation of a text-level reading intervention for aphasic patients}

\author{
Irene Ablinger ${ }^{1,3}$, Ralph Radach ${ }^{2}$, Klaus Willmes ${ }^{3}$, Valentine Silberling ${ }^{4}$ \\ 1 SRH Fachhochchule für Gesundheit Gera, Germany \\ 2 University of Wuppertal, Germany \\ ${ }^{3}$ RTWH Aachen University Hospital, Aachen, Germany \\ ${ }^{4}$ Asklepios Klinik Harburg, Hamburg, Germany \\ irene.ablinger-borowski@srh-gesundheitshochschule.de
}

Text-level reading aloud interventions for aphasic patients with acquired dyslexia have so far been focused exclusively on the improvement of global fluency and the reduction of reading errors. We report the first study using eye tracking methodology to guide individual training and to evaluate performance on the word level before and after therapy. Six patients with mild to moderate aphasia, showing slow reading with uncertainties, selfcorrections and phonological errors, received an intensive intervention (five days per week) over a period of four weeks.

Materials included a series of crime stories (from 400 to over 800 words), followed by multiple-choice comprehension questions. These passages were read repeatedly until a certain level of comprehension-based fluency was attained. Results of pre-post comparisons indicate large increases in reading fluency and accuracy for the entire sample. Critically, there was a substantial transfer effect to untrained passages, which showed somewhat smaller, but still significant, performance improvements. Looking at the individual level, oculomotor behavior changed markedly in all patients, with local fixation patterns becoming more regular with less regressions and less time spends re-reading the same words. Implications for utilizing eye tracking routinely in the planning and on-line evaluation of text-level clinical reading training will be discussed. 


\title{
Relationship between psychopathic personality traits, visual attention and emotion recognition of facial expressions: An eye-tracking study
}

\author{
Maria João de Sousa Seixas ${ }^{1}$, Pedro Rocha Almeida ${ }^{1,2}$, Pedro Vieira Fernandes ${ }^{3}$, \\ Tiago Oliveira Paiva ${ }^{2}$, Lucas Borges da Silva ${ }^{3}$ \\ ${ }^{1}$ School of Criminology, Faculty of Law, University of Porto, Portugal \\ ${ }^{2}$ Laboratory of Neuropsychophysiology, Faculty of Psychology and Educational Sciences, University of \\ Porto, Portugal \\ ${ }^{3}$ Department of Physics and Astronomy, Faculty of Science, University of Porto, Portugal \\ mj.sousa.seixas@gmail.com
}

Psychopathy is a relevant issue in criminology due to its association with serious and violent criminal behavior. Although the evidence of impairments in processing facial expressions in individuals with psychopathic tendencies is not robust, these are considered to be a key feature of psychopathy. Leading us to an apparent paradox: how psychopaths can be described as proficient emotional manipulators if they are not able to identify emotions? This study examined the relationship between psychopathic personality traits and facial emotion recognition. Of the methods used in psychopathy research, eye-tracking remains understudied. This study applied eye-tracking methodology to analyze how affective facial information is processed by individuals when performing a verbal emotion recognition task. Fifty-seven participants were assessed for psychopathic traits and exposed to video clips of emotional facial expressions. Preliminary results indicated that psychopathy is not characterized by behavioral impairments in emotion decoding. Contrary, it was evidenced an enhanced ability to recognize sadness facial expressions in individuals who scored high on boldness, psychopathic interpersonal characteristics such as manipulative, glibness, egocentric and deceitful, influenced by an efficiente selective visual attention to facial affective cues. An enhanced ability to detect sadness would give them a predatory advantage in exploiting, manipulate and deceive others.

\section{The Gap-effect in people with dementia: A longitudinal eye-tracking study}

\author{
Trevor Crawford, Alex Devereaux \\ Lancaster University, United Kingdom \\ t.crawford@lancaster.ac.uk
}

Eye tracking provides a convenient and promising biological marker of cognitive impairment in patients with neurodegenerative disease. Here we report a longitudinal study of saccadic eye movements in a sample of patients with dementia and elderly control participants who were assessed at start of the study and followed up 12 months later. Eye movements were measured in the standard gap and overlap paradigms, to examine the longitudinal trends in the ability to disengage attention from a target. Overall patients with dementia had slower reaction times than the control group. However, after 12 months, both groups showed faster and comparable reductions in reaction times to the gap, compared to the overlap stimulus. Interestingly, there was a general improvement for both groups with more accurately directed saccades and speeding of reaction times after 12 months. These findings point to the value of longer-term studies and follow-up assessment to ascertain the effects of dementia on oculomotor control. 
Posters

\title{
Eye movements and memory
}

\section{Perceptual memory can bias subsequent saccades after natural but not induced systematic endpoint errors

\author{
Paul Zerr ${ }^{1}$, Katharine N. Thakkar ${ }^{2}$, Stefan Van der Stigchel ${ }^{1}$ \\ ${ }^{1}$ Helmholtz Institute, Department of Experimental Psychology, Utrecht University \\ ${ }^{2}$ Brain Center Rudolf Magnus, Department of Psychiatry, University Medical Center Utrecht \\ zerr.paul@gmail.com
}

Previous work has shown that endpoint errors in saccades are largely compensated for in the programming of subsequent saccades. This error compensation is informed by motor vector copies but typically remains incomplete. To investigate compensation for natural fluctuations and induced systematic changes in saccade gain we combined a global saccade adaptation paradigm and a double step saccade task with great stimulus variability. We hypothesized that the deviation between perceptually predicted and actually executed saccades would bias the targeting angle of subsequent saccades. The results show that angular error in second saccades increased linearly with increasing deviation of actual from predicted first saccades but only for natural and not for systematically induced gain error. In line with our hypothesis we observed a directional effect of over- and undershoots in first saccades on angular error in second saccades. No change in this effect or increase in second saccade endpoint errors was observed after saccades had been shortened systematically, suggesting that endpoint errors due to induced adaptation are accounted for in the calculation of following saccades. We show that the visual system is aware of systematic changes to saccade metrics but can be biased by perceptually predicted saccades after natural fluctuation in gain.

\section{Visual working memory resources are dynamically redistributed to saccade targets before saccade onset}

\author{
Edwin S. Dalmaijer ${ }^{1}$, Masud Husain ${ }^{1,2}$ \\ ${ }^{1}$ Department of Experimental Psychology, University of Oxford, United Kingdom \\ 2 Nuffield Department of Clinical Neuroscience, University of Oxford, United Kingdom \\ edwin.dalmaijer@psy.ox.ac.uk
}

In order to interact with the environment, we need to remember where different objects are located, as well as features belonging to those objects. However, visual working memory (VWM) capacity is highly limited. Here we ask how VWM resources are dynamically reallocated during active vision. We presented participants with arrays of Gabor patches of different orientation at varying locations, allowing them to fixate each only once. During the final saccade, the array was replaced by a blank screen, and followed by a Gabor patch with either the same location or orientation as one of those in the original array. Participants were asked to report the corresponding stimulus orientation or location using analogue, continuous response scales. Recall precision for stimuli that had already been fixated was significantly worse than that of stimuli that were about to be fixated - but had not yet been fixated. This effect is unlikely explained by fixation recency or duration, as these did not predict recall precision. Our results support the view that limited VWM resources are dynamically redistributed over relevant parts of the scene, crucially to achieve the highest fidelity for elements that we are about to foveate and potentially interact with. 


\title{
Finding memory load in the eyes
}

\author{
Radha Nila Meghanathan, Cees van Leeuwen, Andrey R. Nikolaev \\ KU Leuven, Belgium \\ radhanila.meghanathan@ppw.kuleuven.be
}

In free eye movement behavior, fixation duration is known to increase with memory load while sensitivity of pupil size to memory load is unexplored. We assessed the extent to which pupil size reflects memory load by presenting two subsequent displays while recording pupil size and eye movements. In the first display, participants searched for 3, 4 or 5 targets among distractors, both in various orientations. In the following display, participants reported whether one of the targets had changed orientation, which was the case in half of the trials. We found that fixation duration reflected the number of fixated targets which was below and above the limit of working memory capacity. In contrast, pupil size did so only when the number exceeded memory capacity. This result indicates that fixation duration reflects both memory load within the working memory capacity and processing load when memory capacity is exceeded, while, pupil size reflects only processing load. Moreover, fixation duration increased with successive fixations for targets but not for distractors, whereas pupil size showed no such distinction. Therefore, fixation duration is a more versatile measure of memory load than pupil size in unconstrained eye movements.

\section{Sleep dependency of episodic memory consolidation - hints from children's eyes.}

\author{
Jing-Yi Wang ${ }^{1,2}$, Frederik D. Weber ${ }^{1}$, Jan Born ${ }^{1}$ \\ ${ }^{1}$ Institute of Medical Psychology and Behavioral Neurobiology, University of Tübingen \\ ${ }^{2}$ Graduate School of Neural \& Behavioural Sciences - International Max Planck Research School \\ jingyi.wang@medizin.uni-tuebingen.de
}

A recent study in human adults indicated sleep's beneficial role for "what-where-when" episodic-like memory utilizing eye tracking. Using the same paradigm we investigate, if the advantage of deeper and longer sleep in school children (8-12 yrs.) further impacts episodic memory consolidation. Children experienced two short episodes one hour apart, each comprising four distinct female faces at different locations in a $3 \times 3$ grid on a PC screen. After encoding, children either slept overnight, or stayed awake during the day. Following a retention of $10.5 \mathrm{~h}$, episodic memory was tested implicitly by tracking eyes and explicitly by oral report. The preliminary data indicated, in line with adults, explicit "what-where-when" memory performance was better in children that slept, while both groups had above chance performance. On the contrary, using the previous implicit measures of spatial and temporal components in the eye exploration pattern did not reflect children's explicit performance. Sensitivity of those measures was addressed by closer matching children's eye behavior to those of adults (i.e. exploration duration and look counts), none of which reflected the features of episodic-like memory. We suggest here that looking behavior in children does not reflect "what-where-when" episodic-like memory the same way as in adults. 
Posters

\title{
Verbal and spatial encoding show different eye movement strategies in a working memory task
}

\author{
Sebastian F. Henschke ${ }^{1}$, Elke B. Lange ${ }^{1}$, Laura Rerko ${ }^{2}$ \\ 1 Max-Planck-Institute for Empirical Aesthetics, Germany \\ 2 University of Zurich \\ sebastian.henschke@aesthetics.mpg.de
}

When processing visual information saccades are generated to focus the gaze plus attention on a stimulus. Since foveally focused, these stimuli are not only perceived better but also processed faster. It is conclusive to assume eye movements to benefit memory encoding in a visually presented memory task. However, it has been demonstrated that visuo-spatial encoding does not recruit the gaze as much as verbal encoding does as indicated by decreased fixation probability, saccadic amplitude and reduced saccadic reaction times. Given these diverging eye movement patterns, what will happen when verbal as well as spatial features have to be encoded at the same time?

We serially presented pairs of letters, bigrams, at different positions on a ring and asked participants to either recall the letters comprising each bigram (verbal task), the locations on the ring at which these were presented (spatial task), or to recall both (binding) in the order of presentation. Thirty participants completed a session comprising 90 trials of 30 trials per task and their eye movements were recorded. Recall accuracy analysis was combined with parameters of the recorded eye movements. Preliminary results confirm former findings and show a complex interaction in the binding task.

\section{Learning set size influences pupil dilation during retrieval} Péter Pajkossy ${ }^{1,2}$, Attila Keresztes ${ }^{3}$, Mihály Racsmány ${ }^{1,2}$

1 Hungarian Academy of Sciences, Research Group on Frontostriatal Disorders, Budapest, Hungary

2 Department of Cognitive Science, Budapest University of Technology and Economics, Budapest, Hungary

3 Max Planck Institute for Human Development, Berlin, Germany ppajkossy@cogsci.bme.hu

It is assumed that pupil dilation indexes both mental effort (Kahneman, 1973) and processes underlying successful episodic memory retrieval (Goldinger\&Papesh, 2012). In the present study we used pupillometry to investigate whether the size of an episodic memory set influences the magnitude of effort during retrieval. Participants took part in several blocks of paired-associate learning with subsequent testing. In each block, participants first learned weakly related word-pairs (e.g. cotton-kayak), then subsequently, they were presented with the left-side word of each word-pair, and were required to retrieve its pair (e.g. cotton-?). As experimental manipulation, we varied the size of the learning set by manipulating the number of word-pairs in each block. There were eight blocks with two word-pairs, four blocks with four word-pairs, and two blocks with eight word-pairs. It was found that pupil dilation during correct retrieval of target words was significantly larger if the given block contained eight word-pairs, in comparison with the other two conditions with either four or two word-pairs in a block. Our results suggest that the size of an episodic learning set influences the retrieval effort of a given target item during retrieval.

(This work was supported by the KTIA NAP Grant (ID: 13-2-2014-0020)). 


\title{
Working memory matters, near-hand postures do not: Why are the effects of working-memory load and interference-control demands on pupil dilation not influenced by the near-hand effect?
}

\author{
Christian Scharinger, Peter Gerjets \\ Knowledge Media Research Center Tuebingen, Germany \\ c.scharinger@iwm-kmrc.de
}

Recent research has shown that near postures support executive functions like set shifting or interference control (Davoli, Du, Montana, Garverick, \& Abrams, 2010; Weidler \& Abrams, 2013). For example, in a flanker task paradigm, placing the hands near the stimuli for responding reduced the flanker interference effect (near-hand effect) whereas the opposite hold true for placing the hands far the stimuli.

In the current study we were interested whether this near-hand effect would also occur for load on another executive function, namely working memory updating (WMU). Using a combined n-back-flanker task, we simultaneously manipulated load on WMU (0-back versus 2-back), demands on inhibitory control (congruent versus incongruent flanker stimuli), and posture (near-hand versus far-hand). Reaction times and pupil dilation served as dependent measure. We expected the near-hand effect to be visible as less pronounced pupil dilation for demands on inhibitory control as well as load on WMU in the near-hand as compared to the far-hand condition. Our results revealed a) increased pupil dilation for load on WMU and demands on inhibitory control, b) an interaction between the two, and c) no effect of posture. We will discuss these results and possible explanations.

\section{Tracking the eyes for sleep's benefit in episodic memory consolidation. Frederik D. Weber, Jan Born \\ Institute of Medical Psychology and Behavioral Neurobiology, University of Tübingen frederik.weber@uni-tuebingen.de}

Episodic events are at the core of our memory and entail information of "what" was involved, "where" and "when" that is bound in common context. Despite abundant evidence for sleep's beneficial role in memory consolidation little is known specifically for episodic memories. A recent study found that sleep benefits "what-where-when" episodic-like memory of two induced episodes that were one hour apart, each presented four distinct female faces in a $3 \times 3$ grid of locations on a PC screen. Recall 10 hours later after either normal sleep or wakefulness comprised presentation of two faces from each episode (temporal), and one each changed locations (spatial). Memory was indicated by scoring spontaneous eye exploration behavior on all faces, whereas an interaction of spatial and temporal features indicated stronger episodic memory after sleep that was correlated with an additional explicit assessment. However, it remains unknown how (qualitatively) and how much (quantitatively) such memory representations change during sleep-mediated consolidation processes. Here we amend the previous study with better qualitative assessment and timecourse analysis showing the evolution of looking behavior from pre-sleep performance. Ongoing work will be present on how sleep influences episodic memory features and how this can be indicated by eye tracking and sleep architecture. 
Posters

\title{
Parallel Modulation of Pupil Diameter and EEG Occipital Alpha Power by Attentional Engagement
}

\author{
Zeeshan Syed, Karina J. Linnell
}

Goldsmiths, University of London, United Kingdom z.syed@gold.ac.uk

Pupil diameter (PD) has been linked to attentional engagement. Recent work has highlighted the relationship between PD and EEG occipital alpha (Hong et al. 2014). Here, we sought to further investigate the relationship between PD and EEG occipital alpha by comparing their response to varying levels of attentional engagement.

PD and EEG data were simultaneously collected from participants who took part in two tasks that differed primarily in the amount of engagement required (Smallwood et al. 2011). In both tasks, participants were presented with a sequence of numbers. The Working Memory (WM) task encouraged task engagement by requiring participants to remember all numbers and, when prompted, report on the previous number in the sequence. In contrast, the Choice-Reaction-Time (CRT) task encouraged disengagement given that the target for report was the number currently on-screen.

During the WM task, incorrect trials were preceded by elevated baseline levels of both PD and alpha power while, in the CRT task, baseline levels of PD and alpha in correct and incorrect trials were indistinguishable. These results show that occipital alpha and PD are similarly associated with attentional engagement due, potentially, to the shared relationship both PD and EEG occipital alpha have with cortical excitability. 


\title{
Intermodal influences
}

\author{
Tactile stimulation biases in free viewing behavior \\ José Pablo Ossandón ${ }^{1}$, Tobias Heed ${ }^{2}$, Peter König ${ }^{1}$ \\ ${ }^{1}$ University of Osnabrück, Germany \\ 2 University of Hamburg, Germany \\ jossando@uos.de
}

We evaluated the effect of irrelevant tactile stimulation on humans' free-viewing behavior during the exploration of complex static scenes. Specifically, we address the questions of (1) whether task-irrelevant tactile stimulation presented to subjects' hands can guide visual selection during free viewing; (2) whether tactile stimulation can modulate visual exploratory biases independent of image content and task goals; and (3) in which reference frame these effects occur.

Forty-seven right-handed subjects participated in a free viewing task while their eye movements were recorded, and tactile stimulation to uncrossed or crossed hands was provided at random moments of visual exploration.

Tactile stimulation resulted in a long-lasting modulation of visual orienting responses. Subjects showed a well-known leftward bias during the early exploration of images, which was modulated by tactile stimulation presented at image onset. Both with uncrossed and crossed hands, tactile stimulation biased visual orienting globally toward the space ipsilateral to the stimulated hand, but did not elicit reactive saccades to the actual location of the stimulated hand. In conclusion, our results indicate that tactile-to-visual cross-modal cues produce spatially global and long-lasting orienting responses, which appear to be coded exclusively in external coordinates.

\section{Modulation of the vestibulo-ocular reflex by transcranial direct current stimulation}

Kiki Arkesteijn

Erasmus MC, Netherlands, The

arkesteijn.kiki@gmail.com

The vestibulo-ocular reflex (VOR) is an eye movement that is used to stabilize images on the retina during head movement. The VOR pathway has been studied extensively, and adaptation of this reflex is widely used as a cerebellar learning paradigm. Here we investigate the ability of direct current stimulation (DCS) to modify the rate of this adaptation. DCS is a non-invasive brain stimulation technique that changes excitability of neurons by applying weak current to the skull, or in animals directly to the brain's surface. It is believed that DCS changes behavioural outcome measures by facilitating or inhibiting plasticity. We investigate the precise forms of synaptic plasticity that mediate these effects by studying the effects of stimulation in mice lacking the cellular mechanisms for either LTD or LTP at the parallel fibre- Purkinje cell synapse. We also investigate the effect of different intensities of stimulation in wild type mice. Preliminary results shows that DCS is capable of altering the rate of VOR adaptation in wild types in a polarity and intensity dependent manner. Additionally the effects of stimulation are modified in mice in which specific forms of synaptic plasticity have been genetically ablated. 
Posters

\section{Remote eyetracker recovery and performance during head rotation}

Roy S. Hessels ${ }^{1,2}$, Tim H. W. Cornelissen ${ }^{1,2}$, Chantal Kemner ${ }^{1,2,3}$, Ignace T. C. Hooge ${ }^{1}$

${ }^{1}$ Department of Experimental Psychology, Helmholtz Institute, Utrecht University, The Netherlands

2 Department of Developmental Psychology, Utrecht University, The Netherlands

${ }^{3}$ Brain Center Rudolf Magnus, University Medical Centre, Utrecht, The Netherlands royhessels@gmail.com

What are the decision criteria for choosing an eyetracker? Often the choice is based on specifications by the manufacturer of the validity (accuracy) and reliability (precision) of measurements that can be achieved using a particular eyetracker. These specifications are mostly achieved under optimal conditions-for example, by using an artificial eye or trained participants fixed in a chinrest. Research, however, does not always take place in optimal conditions: For instance, when investigating eye movements in infants, school children, and certain patient groups. We modeled movements often seen in infant research in two behaviors: (1) looking away from and back to the screen, to investigate eyetracker recovery, and (2) head orientations, to investigate eyetracker performance with nonoptimal orientations of the eyes. We investigated how eight eyetracking setups by three manufacturers (SMI, Tobii, and LC Technologies) coped with these modeled behaviors in adults, and reported on their rates of data loss and systematic offsets during shifted head orientations. We conclude that (prospective) eye-movement researchers who cannot restrict movement or nonoptimal head orientations in their participants might benefit from testing their eyetracker in nonoptimal conditions. Additionally, researchers should be aware of the data loss and inaccuracies that might result from nonoptimal head orientations.

\section{Seeing what you hear: two exploratory studies using eye movement registrations to reveal the time course of speech understanding}

\section{Monique Lamers}

VU University Amsterdam, Netherlands, The m.lamers@vu.nl

To examine how eye movement registration may help to reveal the time course of cognitive processes involved in speech understanding two exploratory studies were set up. In the first study, eye movements were registered while the hearer was listening to words and looking at a set of four pictures including a picture representing the auditory stimulus. Such a multimodal set-up makes speech audiometry suitable for preliterate individuals, such as young children. Although there is room for improvement of the paradigm, first results indicate that eye movement registration forms a valuable asset to traditional speech audiometry as it provides detailed information about the exact phonemes that were understood in the word. A second paradigm was set up to investigate whether language users with a cochlear implant use the same cues as normal hearers do to discriminate two phonemes that differ in voice-onset-time (VOT) using a / ba/-/pa/ continuum. Initial analyses indicate that some $\mathrm{CI}$ hearers, but not all, use different criteria within the continuum than normal-hearers; others seem to use a different cue than the VOT. Further analyses, including the analysis of pupil dilatation will reveal possible differences in the real-time cognitive effort between CI and normal hearing listeners. 


\section{Can We Entrain the Saccade Timer to Musical Tempo? Jonathan P. Batten ${ }^{1}$, Gustav Kuhn ${ }^{2}$, Tim J. Smith ${ }^{1}$ \\ ${ }^{1}$ Birkbeck, University of London, United Kingdom \\ 2 Goldsmiths, University of London, United Kingdom j.batten@bbk.ac.uk}

When listening to music people often move their bodies rhythmically in time with the accents of the beat. This embodied response; in addition to physiological measures of arousal, is modulated by changes in musical tempo. Eye movements are thought to be subject to an underlying timing mechanism, modulated by processing demands. This study investigated the influence of variation in musical tempo on eye movement timing.

Participants were tasked with a gaze contingent visual search task, in which they were required to move their eyes clockwise around 12 equidistant points resembling a clock face. Their task was to quickly and accurately identifying the target letter ' $\mathrm{L}$ ' from the distractor ' $\mathrm{T}$ '. Whilst completing this task, participants listened to instrumental music at 80 and 120 beats per minute, or silence. A further beat tapping task to the music provided a measure of beat sensitivity.

Beat tapping ability showed large variation across individuals, however this ability and the musical manipulation did not manifest in differences in eye movement timing. This may suggest a robust idiosyncratic timing mechanism not influenced by music, and variation in musical tempo. Future research will employ auditory tempi that are closer aligned to the natural movement timing.

\section{Up-down asymmetry in visually induced self-motion perception (vection) \\ Yasuhiro Seya, Hiroyuki Shinoda, Yoshiya Nakaura \\ Ritsumeikan University, Japan \\ yseya@fc.ritsumei.ac.jp}

Research has reported a close link between visually induced self-motion perception (vection) and optokinetic nystagmus (OKN) by showing stronger vection in stimulus conditions that are more effective in eliciting OKN than in those that are not. In the present study, we investigated whether vection would reflect up-down asymmetry in vertical OKN, i.e., larger OKN responses for upward motion than for downward motion. In Experiment 1, participants viewed a vertically moving random-dot pattern, with or without a fixation stimulus. During the presentation of the moving dots, the participants reported vection by using a joystick, and after each trial, they rated the vection magnitude. In Experiment 2, the time course of the vection magnitude was examined in the presence of the fixation stimulus. Experiment 1 showed stronger vection for the upward motion than for the downward motion, and for higher stimulus velocities, irrespective of the fixation stimulus. In Experiment 2, the up-down asymmetry in vection manifested progressively during the later part of the stimulus presentation period and its onset became more rapid with increasing stimulus velocity. These results not only support a close link between vection and OKN, but they also suggest an overlap in the mechanisms that generate vection and $\mathrm{OKN}$. 
Posters

\title{
Learning and adaptation
}

\section{The Effects of Synchronized Cues and Learners' Expertise on Multimedia Learning: An Eye Movement Study}

\author{
Fuxing Wang, Heping Xie \\ Central China Normal University, People's Republic of China \\ fxwang@mail.ccnu.edu.cn
}

\begin{abstract}
Cueing and expertise affect learners' bottom-up and top-down processing respectively in multimedia learning. Previous studies pay little attention on the temporal synchronization between the presentation of cues and audio interpretation.

Three types of cueing, advanced, synchronized, and delayed, were manipulated. For the advanced and delayed cues, highlighted parts were presented $2.6 \mathrm{~s}$ before or after the corresponding audio interpretation. High and low prior knowledge learners were recruited as participants and randomly assigned to three cueing conditions. SMI RED 500 Eye tracker was used to record eye movement data.

The results showed that learners of synchronized cues condition had higher scores in all of the three tests (retention, transfer, and matching tests) and lower perceived difficulty of the material than unsynchronized groups. The initial 3 seconds eye movement data showed that fixation time and first fixation duration of synchronized cues were longer, and the glance count and fixation count were much more than delayed and advanced cueing groups. However, there was no interaction between synchronized cues and learners' expertise. We only found that high-prior knowledge learners performed better and perceived lower difficulty. Synchronized cueing can effectively guide learners' attention, help learners construct the representation between audio words and animations, and improve learning performance.
\end{abstract}

\section{Framing Gameplay Experience with Eye Movement Based Adaptation Katharina Spiel, Sven Bertel \\ Bauhaus-Universität Weimar, Germany \\ katharina.spiel@tuwien.ac.at}

Gameplay Experience is influenced by many different aspects that a game can have. The core ludic mechanisms, graphical elements (or lack thereof), platforms, potentially narrative storylines, genres and other aspects have been analysed in depth individually and combined. This work looks at games from a different angle: How do assumptions of technological novelty and its framing contribute to games? For this purpose we created (N)EMtris, a Tetris version adapting its difficulty in terms of speed and block choosing algorithm to the eye movements of the individual player. The eye movements are abstracted from how a player looks at a game (such as the percentage of relevant areas or the speed of change of focus). In a user study players were divided into two groups: Those, which were told that the game uses this kind of adaptive technology and those who were not told. Both groups played games with and without eye movement based adaptation. Results of the study indicate that performance is more affected by eye movement based adaptation, that framing and adaptation have an influence on gameplay experience (at least in some settings), and that framing shapes expertise as measured by eye movements in conventionally adapted games. 


\title{
Saccadic adaptation is associated with starting eye position
}

\author{
Svenja Gremmler, Markus Lappe \\ University of Muenster, Germany \\ svenja.gremmler@uni-muenster.de
}

Saccadic adaptation is the ongoing motor learning process that keeps saccades ending accurately on their targets. Latest research showed that amplitude modification that was induced in a saccade at one location transfers incompletely to saccades at other locations. Thus an eye position signal needs to be utilized in the adaptation mechanism. In our current study we investigated if the specificity regards to the initial or the final eye position of the saccade and hence which signal is taken into account by the adaptation mechanism. We adapted a saccade of $15 \mathrm{deg}$ amplitude that was started at a constant position and measured the transfer of adaptation to four different test saccades of $10 \mathrm{deg}$ and $20 \mathrm{deg}$ amplitude, matching either the starting position or target position of the adapted saccade. We found significant less transfer of adaptation to test saccades with the same target position than to test saccades with same starting position like the adapted saccade for both, the $10 \mathrm{deg}$ and 20 deg test saccades. This indicates that saccadic adaptation is specific to the saccadic starting position during adaptation and that the eye position signal from the beginning of the saccade is stored and utilized in the adaptation mechanism.

\section{Gait assessment - development of skilled gaze behavior in physiotherapy students}

\author{
Carina Prammer, Anna-Maria Gorgas \\ St. Poelten University of Applied Sciences, Austria \\ pt121027@fhstp.ac.at
}

Gait assessment is an important part in physiotherapy work. Due to the gait's complexity and the limited time available, a certain level of experience is required to accurately determine deviations from normal gait. Acquiring this skill poses a great challenge to physiotherapy students (PTSt). The objective of the present study was to analyze and compare the gaze behavior of PTSt with different educational levels to determine experience-related changes in applied gaze strategies.

33 PTSt (11 students of each year of training) and 11 students of different studies (control group, CG) participated in this study. Eye-tracking data were recorded while participants watched a $30 \mathrm{sec}$ video sequence showing a patient with gait aberrations (using a stationary eye-tracking system, SMI).

The outcomes revealed no statistically significant data. However, averaged gaze fixation durations were longer in more experienced participants (2nd year of education: $541,79 \mathrm{~ms} \pm 194,4$; 3rd year: $494,56 \mathrm{~ms} \pm 124,7)$ compared to those of novices and CG (novices: 435,36ms $\pm 112,86$; CG: $426,45 \mathrm{~ms} \pm 175,34$ ). Novices showed the highest number of saccades. These findings conform to the outcomes of previous studies on experts' gaze behavior in related fields and may indicate a transition due to experience. Further research is required to obtain reference values for the gaze behavior of physiotherapy experts. 
Posters

\author{
Learning human gazing behavior with a simple neural network \\ Mohamed Ashraf ${ }^{1}$, Florian Röhrbein ${ }^{2}$ \\ 1 German University Cairo \\ 2 Technische Universität München \\ mohamed.ashraf.213@gmail.com
}

The goal of our project was to train an artifical neural network model for the prediction of salient regions in static and in dynamic natural scenes. For this, subjects were shown greyscale static images as well as fixed camera dynamic scenes and their eye movements and fixations were recorded to create saliency heatmaps. Spatial and temporal features such as contrast, brightness, gradient information and distance from the center were collected from the areas around salient and non-salient points. The extracted features were fed into a feed-forward neural network with two hidden layers for training. Around 18000 labeled locations were used for training and another 6000 were used for testing. The goal was to minimize the cross entropy error function which is often used for classification problems. After training the neural network was able to detect the most salient regions in static and dynamic scene. This output had an accuracy of around $85 \%$ on average with slighter better performance for dynamic natural stimuli. The result shows that a simple neural network can be succesfully trained to learn and predict basic human gazing behavior.

\title{
Do saccades adapt to the distance between targets in visual search? Christof Körner $^{1}$, Margit Höfler ${ }^{1}$, lain D. Gilchrist ${ }^{2}$ \\ ${ }^{1}$ Universität Graz, Austria \\ 2 University of Bristol, UK \\ christof.koerner@uni-graz.at
}

In the present experiment we were interested in whether the oculomotor system can adapt to the distance between two targets in a multiple-target visual search. Thirty-five participants searched displays with 30 items each for two identical targets. We manipulated the distance between the targets blockwise in three conditions: The distance could be small, large or random. We investigated whether participants would become aware of this distance contingency and whether the oculomotor system would adapt to it. While the time to find the first target did not differ between conditions, participants fixated the second target faster in the small distance compared to the large and random distance condition. In a postexperimental interview, almost half of the participants reported that they noticed the small distance contingency. However, only four participants reported that they noticed the long distance contingency. In a preliminary analysis we showed that the saccade amplitude that followed the first target was somewhat longer for the long distance condition compared to the other conditions. This result may indicate that the oculomotor system adapts flexibly to the respective distance between targets, even though participants do not become fully aware of such contingencies. 


\title{
Monitoring the Learning Process when Learning Radiology by Case Comparisons
}

\author{
Ellen Kok ${ }^{1}$, Anique de Bruin ${ }^{1}$, Jimmie Leppink ${ }^{1}$, Simon Robben $^{2}$, Jeroen van Merriënboer ${ }^{1}$ \\ 1 Maastricht University, School of Health Professions Education, Department of Educational \\ Development and Research \\ 2 Department of Radiology, Maastricht University Medical Centre \\ e.kok@maastrichtuniversity.nl
}

Much research in education focuses on outcomes (e.g. grades). Eye tracking, however, allows for the investigating of learning processes. We investigated how participants use case comparisons for learning the interpretation of radiographs, a complex visual task. Three types of comparison were tested against a sequential control condition: comparison of images from different patients with the same disease (same-disease comparison), comparison of images showing different diseases (different-disease comparison) and comparison of disease-images with normal images (disease/normal comparison).

We randomly assigned 84 medical students to one of 4 conditions and had them review 6 cases for each of 8 different diseases on radiographs, while their eye movements were being measured using an SMI RED 250. Consequently, participants took 2 tests that measured diagnostic performance and ability to locate diseases respectively.

Participants in the same-disease and different-disease conditions were most efficient, reaching the same performance but in less time. We found comparison in $91 \%$ of all trials in the comparison conditions, but there was no relationship between the number of comparisons and performance. Not many comparisons were made between abnormalities and normal tissue or between two abnormalities, which is peculiar given that students were required to study the appearance of pathology.

\section{Measuring learning strategies: An eye-tracking approach Tristan Haselhuhn, Sebastian Schmid, Klaus-Peter Wild University of Regensburg, Germany tristan.haselhuhn@ur.de}

The application of learning strategies has repeatedly been shown to promote learning success. In an experiment, we explored the potential of eye tracking methods for the analysis of learning strategies during the reading process. We distinguished between surface- (memorizing of facts) and deep- (understanding and conclusion-oriented) level processing. Learning strategies were triggered by learning process oriented instructions. Participants were 67 student freshmen enrolled in Educational Science, who read two naturalistic newspaper articles on social issues. The double-blind experiment was based on a 3 (deep vs. surface vs. control) x 2 (text order) design. Gaze movements and self-reports of the participants were used as indicators of learning strategies. A $250 \mathrm{~Hz}$ remote eye tracker was used and free head movement were allowed to create an ecologically valid learning situation. Fixations and regressions during the reading process were interpreted based on a content analyses of the newspaper articles. Experimental groups were compared in order to find out whether self-reports and gaze movements can be used as indicators for learning strategies. Finally, we discuss the potential these measurements have to reveal different learning strategies. 
Posters

\title{
The Perceptual Differences between Experienced Chinese Chess Players and Novices: Evidence from Eye Movement
}

\author{
Fuxing Wang, Xiujuan Hou, Zhaohui Duan, Huashan Liu \\ Central China Normal University, People's Republic of China \\ fxwang@mail.ccnu.edu.cn
}

Previous chess studies have found that the experienced chess players are better than novice on memorizing chess board, and show superior perceptual encoding advantage. But whether Chinese chess would have the similar expertise superior advantage? That is the main purpose of this study.

Ten Chinese chess players who were having more than 10 years experience as experienced players. Fifteen college students who could understand the basic rules of Chinese chess were recruited as novices. Real Chinese chess playing-board pictures with 14 pieces on board were materials. Three experiments were carried out to explore the chess board memory, parafoveal processing, and perceptual encoding of chess expertise.

Results found that experienced Chinese chess players could recall much more pieces, had wider saccade span and greater pupil size than novices. Experienced chess players fixated more between pieces rather than on the pieces, could take advantage of parafoveal processing, perceived changed pieces faster and their correct rate were higher than novices.

Consistent with the chess studies, Chinese chess did have expertise superior advantage, memorize much more pieces of chess board, allocate more attention on between pieces based on their chess chunks and templates, can use parafovea to encode information, and superior perceptual encoding advantage.

\section{Eye Movement Based Classification of Expertise in Photo Editing}

\author{
Tandra Ghose $^{1}$, Yannik T. H. Schelske ${ }^{2}$, Takeshi Suzuki ${ }^{3}$ \\ ${ }^{1}$ Department of Psychology, University of Kaiserslautern, Germany \\ 2 Department of Computer Science, University of Kaiserslautern, Germany \\ ${ }^{3}$ Ricoh Institute of Technology, Japan \\ tandraghose@gmail.com
}

Can expert knowledge be modeled by machine-learning algorithms based on eyemovement data (EM) in the domain of photo editing? To investigate this question we recorded EM from 4 experts and 4 novices during two photo editing tasks: set the 1 - contrast or 2-color of a given image to the most aesthetically pleasing one. The stimuli were images acquired from expert photographers that were degraded either in 1-contrast or 2-color along the blue-yellow axis.

Clustering of adjusted-contrast and adjusted-color showed two distinct groups corresponding to the experts and novices. For the experts the adjusted-value was closer to that of the original-image.

A support-vector machine was trained to classify EM-based features (luminance at fixation, luminance-variance in small $(3 \times 3 \mathrm{px})$ or large $(51 \times 51 \mathrm{px})$ region around fixation, color at fixation, color-variance in small/large region) into experts or novices. Classificationaccuracy was significantly higher for the contrast $(60 \%)$ than in color (52\%) adjustment task. Luminance-features were significantly more discriminative during contrast than during color-adjustment, and vice-versa for color-features. Luminance-features were more discriminative (60\% accuracy) than color-features (54\%).

Based on EM-based classification of observer expertise we conclude that EM encode task-relevant information (increased discriminability of color-/luminance-features in color/contrast-based tasks respectively). 


\title{
Cerebellar transcranial Direct Current Stimulation does not affect inward saccadic adaptation
}

\author{
Suzy Margaretha ${ }^{1}$, Eric Avila ${ }^{1}$, Jos N. van der Geest ${ }^{1}$, Maarten A. Frens ${ }^{1,2}$ \\ ${ }^{1}$ Neuroscience, Erasmus MC Rotterdam, The Netherlands \\ 2 Erasmus University College, Rotterdam, The Netherlands \\ suzymargaretha@hotmail.com
}

The cerebellum is crucially involved in eye-movement control and adaptation. Transcranial direct current stimulation (tDCS) is a non-invasive method to modulate neuronal excitability. A previous study from our lab showed that anodal cerebellar tDCS improved performance on an inward saccade adaptation task.

We aimed to replicate this finding and determine whether cathodal tDCS also modulates inward saccade adaptation performance. Subjects $(n=24)$ completed three inward saccade adaptation sessions (10 degrees target amplitude, 30\% intra-saccadic step). Throughout the baseline and adaptation phase (duration: 15 minutes) subjects received either anodal, cathodal or sham tDCS to the right cerebellum (stimulation intensity: $1.5 \mathrm{~mA}$ ).

We found significant saccadic adaptation (i.e., saccade amplitude gradually decreased over 150 adaptation trials). However, we found no differences in performance between anodal, cathodal or sham stimulation. TDCS did also not affect saccade kinematics (peak velocities, durations and saccade latencies). This result is not consistent with the previous finding from our lab that anodal cerebellar tDCS improves performance.

This difference with our labs previous finding might indicate that cerebellar tDCS does not consistently affect saccade adaptation. However, another possibility is that learning effects masked the tDCS effect, since (despite randomization) the amount that subjects learned decreased as a result of session order. 


\title{
Reading
}

\section{Coloring as a syllabification cue in beginning reading: An intervention study}

\author{
Tuomo Häikiö, Jukka Hyönä, Raymond Bertram \\ University of Turku, Finland \\ tuilha@utu.fi
}

Syllabification by hyphens (e.g., hy-phen-a-tion) is a standard procedure in early Finnish reading instruction. However, the effects of hyphenation on beginning Finnish readers' reading and eye movements have not been studied earlier. In Experiment 1, proficient firstgraders read sentences that were concatenated, hyphenated at syllable boundaries or cued with alternate coloring (every second syllable red, every second black). Hyphenation prolonged gaze durations, go-past times and sentence reading times whereas for alternate coloring there was no such change compared to uncued text. These results imply that hyphenation slows down either parallel activation of the syllables or the activation of the wholeword representation. Alternate coloring had no such effect but by virtue of being a novel presentation mode to the beginning readers, it is possible that they cannot utilize it properly as a syllable boundary cue. To examine whether a longer exposure to such material changes the eye movement patterns, in Experiment 2, a group of first-grade children took part in a five-week intervention during which their regular hyphenated $A B C$ book texts were replaced with otherwise identical texts cued with alternate coloring. A control group received the same material with regular hyphenated texts. The results of Experiment 2 will be discussed.

\section{Development of the perceptual span: Nonlinear mixed-effects modeling}

\author{
Johannes M. Meixner, Jochen Laubrock, Anja Sperlich \\ Universität Potsdam, Germany \\ johannes.meixner@uni-potsdam.de
}

The moving window technique is the state-of-the-art method to estimate the perceptual span during normal reading. Reading times and other eye-movement measures are assessed with gaze-contingent windows of varying sizes. The perceptual span is then usually taken to be the largest window size at which normal reading is not impaired, i.e. measures are compared against a full-line condition using post-hoc comparisons (e.g. Rayner, 1986). A more robust approach is needed when using the perceptual span as trait variable in longitudinal studies. As a methodological improvement to the usual approach we implemented nonlinear mixed-effects models, modeling the data as a nonlinear growth curve with three parameters per participant as a random effect: intercept, growth rate, and asymptote. These parameters can be used to trace development of the perceptual span and estimate its stability. We applied the model to a sample of 129 German elementary school children assessed at two time points, beginning in grades 2 and 3 . Results suggest that the perceptual span develops after basic reading skills have been mastered, and that is a fairly stable indicator of reading performance thereafter. In sum, non-linear mixed models offer a promising approach to describe developmental differences in the perceptual span. 


\section{When negative information can't be ignored: Age-related differences in emotion word processing in younger and older adults \\ Eva Fröhlich, Jana Lüdtke \\ FU Berlin, Germany \\ eva.froehlich@fu-berlin.de}

Previous behavioral and electrophysiological studies on single word recognition reported enhanced processing for emotional words compared to neutral ones, a finding that has been replicated for words within sentences. Although measuring eye movements during reading is an established technique to explore perceptual and cognitive aspects of lexical processing only one study examined the influence of a word's emotional qualities on fixation times. Scott et al. (2012) confirmed the processing advantage for emotional compared to neutral words by measuring shorter eye fixation times. Here we wanted to replicate those findings by presenting short texts containing negative, neutral or positive adjectives and explore possible age-related differences. Typically older adults show a positivity bias, they preferentially allocate attention to positive stimuli. We hypothesized that this bias influences eye movements of older adults when reading emotional words in sentences. We therefore compared mean number of fixations, fixation durations and backward regressions of 25 younger and 25 older adults $(\mathrm{M}=26.0 ; 69.5$ years) for whole texts and emotional/neutral adjectives. Older but not younger adults showed more and longer fixations and rereading for short texts containing negative adjectives. Results are discussed with respect to classical age-related changes in reading and in emotion regulation.

\section{Eye movements during the reading of coherent and incoherent sentences: Differential effects for early and late measures}

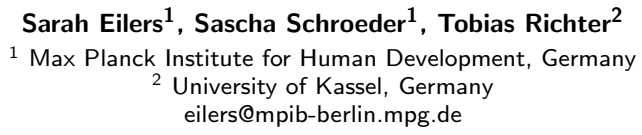

1 Max Planck Institute for Human Development, Germany

2 University of Kassel, Germany eilers@mpib-berlin.mpg.de

Eye movements can be used to study the time course of reading processes. Reading comprehension relies on the integration of sentences into a given discourse context. Linguistic markers such as connectives guide readers towards a coherent interpretation of the text. In German, connectives differ in their syntactic behavior: They introduce either a coordinated or subordinated sentence structure. Crucially, German subordinated clauses follow the canonical word order while coordinated clauses do not. We investigated the online processing of coherent and incoherent discourse depending on the type of connector used. German adults read 40 minimal, three-sentence discourses. The second sentence included a connector varied by selection restriction (coordinated, subordinated) and semantic fit to the discourse context (coherent, incoherent).

Our results showed main effects of the incoherence and subordination conditions in total reading times, indicating explicit rereading. Further, coherence effects for subordinated, but not for coordinated clauses were obtained for early measures (first fixation duration and first-pass time). We interpret this difference in terms of canonicity: A subjunctive introduces the same semantic restrictions as a coordinative, but selects the more frequent word order. Our results suggest that the canonical word order facilitates the integration of a linked proposition into the preceding discourse context. 
Posters

\title{
Eye movement behaviour during the reading of spaced and unspaced Chinese text: Effects of adult age
}

Victoria A. McGowan ${ }^{1}$, Jingxin Wang ${ }^{2}$, Lin $\mathrm{Li}^{2}$, Sha $\mathrm{Li}^{2}$, Fang $\mathrm{Xie}^{2}$, Min Chang ${ }^{2}$, Sarah J. White $^{1}$, Kevin B. Paterson ${ }^{1}$

${ }^{1}$ University of Leicester, United Kingdom

2 Tianjin Normal University, China

vm88@le.ac.uk

Written Chinese consists of a sequence of logographic characters which have no spaces to demarcate word boundaries. It is well established that inserting spaces between words in Chinese produces little effect on the reading of young adults (aged 18-30). However, research has shown that older readers (aged 65+) of alphabetic languages particularly benefit from the availability of interword spaces, therefore the present study tests whether this is also the case for older Chinese readers. The eye movement behaviour of young and older Chinese adults was examined while reading texts that were either unspaced (normal), had spaces inserted between words, or had spaces inserted randomly to create nonwords. Sentence-level analyses revealed effects of these spacing manipulations on a broad range of eye movement measures, resulting in an overall cost in sentence reading times for nonwordspacing, but not word-spacing, for both young and older adults. However, more detailed analyses of two-character target words showed age differences in the effects of inserting spaces between words. Importantly, the results indicate that there may be subtle differences in how interword spaces modulate eye movement behaviour for young and older adult readers of Chinese.

\section{Eye movement parameters in reading syntactically ambiguous} sentences in Russian language

\author{
Victor N. Anisimov ${ }^{1}$, Anna S. Kiseleva ${ }^{1}$, Olga V. Fedorova ${ }^{2}$, Alexander V. Latanov ${ }^{1}$ \\ ${ }^{1}$ Dept. of Neurobiology, M.V. Lomonosov State University, Moscow, Russian Federation \\ 2 Dept. of Theoretical and Practical Linguistics, M.V. Lomonosov State University, Moscow, Russian \\ Federation \\ viktoanisimov@yandex.ru
}

Eye movement parameters in reading syntactically ambiguous sentences with relative clause were studied. In experiments the subjects read locally and globally ambiguous sentences (with one and two interpretations respectively) and sentences without ambiguity (control). In Russian language as well as in other languages the parsing of both globally and locally ambiguous sentences slows down the reading compared with parsing of control sentences. This occurs because of increasing of fixation duration and frequency of regressive saccades executed for rereading the ambiguous phrase. Moreover the subjects read globally ambiguous sentences slower than locally ambiguous sentences. The domination of early/late closure principle in certain language determines fewer efforts for disambiguation of syntactically ambiguous sentence as provided by such domination. This determination closely relates with eye movement parameters during reading ambiguous sentences in languages with domination of late (e.g. English) and early (e.g. Spanish) closure. In Russian language reading time of locally ambiguous sentences with early closure was shorter than reading time of sentences with late closure because of more fixations in latter case. Thus the domination of early closure in Russian language determines eye movement parameters in reading syntactically ambiguous sentences. Supported by Russian Foundation of Basic Research (project No. 15-04-05745). 


\title{
Character positional frequency effects on Chinese word segmentation are independent from neighbourhood size
}

\author{
Feifei Liang ${ }^{1}$, Yongsheng Wang ${ }^{1}$, Hazel I. Blythe ${ }^{2}$, Chuanli Zang ${ }^{1}, X_{\text {Xejun Bai }}{ }^{1}$, Simon P. \\ Liversedge $^{2}$ \\ 1 Tianjin Normal University, P.R.China \\ ${ }^{2}$ University of Southampton, UK \\ feifeiliang_329@126.com
}

Chinese text is normally printed as a continuous string of characters without spaces between words; thus, a key theoretical question is how Chinese readers segment and identify words during reading. Previously, we reported that the positional frequencies of word's constituent characters are used as a segmentation cue when learning new words that are encountered during silent sentence reading (Liang et al., 2013). In that study, however, it was not possible to disentangle the contributions of character positional frequencies and neighbourhood sizes. In the present experiment, participants' eye movements were recorded as they read sentences containing pseudowords that were manipulated for the positional frequencies of their two constituent characters, whilst neighbourhood size was controlled. Target pseudowords were either balanced (neither character had a strong bias towards occurring at the beginnings or ends of words), congruent (both characters were in their most commonly occurring within-word position) or incongruent (both characters were in an unusual within-word position). We also manipulated whether these sentences were presented in a word-spaced, or a traditional unspaced format. Critically, the results showed clear effects of character positional frequencies, demonstrating that Chinese readers are sensitive to this information as a cue to the likely locations of word boundaries.

\section{Effects of word frequency and visual complexity on eye movements of young and older Chinese readers}

\author{
Chuanli Zang ${ }^{1}$, Manman Zhang ${ }^{1}$, Xuejun Bai ${ }^{1}$, Guoli Yan ${ }^{1}$, Kevin B. Paterson ${ }^{2}$, Simon P. \\ Liversedge $^{3}$ \\ 1 Tianjin Normal University, China \\ ${ }^{2}$ University of Leicester, UK \\ ${ }^{3}$ University of Southampton, UK \\ dedipsy@yahoo.com
}

Research using alphabetic languages shows that, compared to young adults, older adults employ a risky reading strategy in which they are more likely to guess word identities and skip words to compensate for their slower processing of text. However, little is known about how aging affects reading behavior for naturally unspaced, logographic languages like Chinese. In the present study, participants read sentences containing a single Chinese character word that had a high or low frequency of usage and was constructed from either visually simple or complex. Frequency and complexity produced similar patterns of influence for both age-groups on skipping rates and fixation times for target words. However compared to the young adults, the older adults made more and longer fixations and more forward and backward eye movements overall. They also fixated the target words for longer, especially when these were visually complex. Crucially, the older adults skipped words less and made shorter progressive saccades. Therefore, in contrast with findings for alphabetic languages, older Chinese readers appear to use a careful reading strategy according to which they move their eyes cautiously along lines of text and skip words infrequently, in order to compensate for increased difficulty processing word boundaries in Chinese. 
Posters

\author{
Exploring the Lack of a Disfluency Effect during Reading \\ Alexander Strukelj ${ }^{1}$, Katharina Scheiter ${ }^{2}$, Marcus Nyström ${ }^{1}$, Manuel Oliva ${ }^{1}$, Kenneth \\ Holmqvist $^{1}$ \\ ${ }^{1}$ Lund University, Sweden \\ ${ }^{2}$ Knowledge Media Research Center, Tuebingen \\ alexander.strukelj@englund.lu.se
}

An eye-tracking study with 60 native Swedish speakers (18-30 years) was conducted to investigate the positive effects on learning outcomes by visually degrading learning materials, as predicted by the disfluency effect [e.g., Cognition, 118(1), 111-115 (2011) doi:10.1016/j.cognition.2010.09.012]. Slightly low-pass filtered text was used as disfluency manipulation and compared with unfiltered text. The text was presented on four separate text presentation screens (TPSs), and comprehension was tested using free recall and a multiple-choice comprehension test given 30 minutes later. Working memory capacity (WMC) was also assessed. The disfluency manipulation had no effect on learning outcomes and was not moderated by participants' WMC. However, total reading times on each word were shorter in TPS 1 and 2 in the disfluent condition compared with the control, but longer in TPS 3 and 4. This significant interaction was also found for number of revisits. Thus, subtle visual degradation initially decrease reading times, but increase reading times over time, likely due to adaptation. Average fixation durations were not significantly affected, so it is likely that the processing level was constant, with differences in processing duration only. As no learning benefits were found, these results suggest that mainly visual processing is affected by disfluency manipulations.

\title{
Eye-tracking evidence that get-passives imply Patient control
}

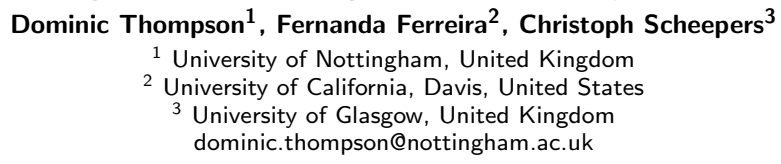

English provides two alternatives to form the passive-voice: BE (was paid) and GET (got paid). Literature provides many suggestion of what functionally separates the two forms, though there is little agreement. One commonly cited idea is that get-passives imply control or intention of the Patient (person that 'undergoes' the action).

We tested this experimentally via a reading eye-tracking study. Participants read short stories. In each story the critical sentence described an event using be-passive or get-passive. The stories implied varying levels of control: either the Patient of the event succeeded in making it happen (successful-control), failed to prevent it from happening (failed-control), or had no specific intentions (neutral-control).

Standard reading measures were taken. A significant interaction was observed in Regression-Path-Duration (RPD) and Number-of-Fixations (NoF). When be-passive was used, the 'failed-control' condition was easiest to process (shorter RPD, smaller NoF); when get-passive was used, the 'failed-control' condition was hardest to process (longer RPD, greater NoF), while 'successful-control' was easiest.

This indicates get-passives are preferred when the Patient is in control of an event, and succeeds in controlling the outcome. Be-passives are not used in the absence of control as such, but rather in situations where attempting to control an outcome fails. 


\title{
The Contribution of Executive Function Components Varies Across the Timeline of Reading
}

\author{
Regina Henry ${ }^{1}$, Victor Kuperman ${ }^{1,2}$, Julie A. Van Dyke ${ }^{2}$ \\ ${ }^{1}$ McMaster University, Canada \\ 2 Haskins Laboratories, USA \\ henryr@mcmaster.ca
}

Executive functions are domain-general cognitive processes that enable and control goaloriented behaviour (Banich, 2009; Miyake \& Friedman, 2012). Deficits in three executive function components have been proposed to contribute to lower reading proficiency: inhibition (Pimperton \& Nation, 2010), updating (Palladino et al., 2001; Rapp \& Kendeou, 2007) and shifting (van der Sluis et al., 2004). However, our study suggests that these contributions vary in magnitude and temporal locus. Ninety-one proficient readers completed a representative battery of executive function tests and their scores were correlated with individual eye-movement data recorded during reading texts for comprehension. Updating ability affected the whole time-course of reading. Participants with lower updating performance had longer first pass, second pass and total reading times, a higher refixation rate and lower skipping rate, indicating continuous difficulty in integrating the new material with prior mental representation of the text. Participants with lower shifting performance, appeared to have difficulty at later stages of reading (higher refixation rate and longer second pass reading times), supporting the hypothesis of sequential shifts of attention (Reichle et al., 2006). Inhibition tasks did not show a stable effect on eye-movements, which is inconsistent with a hypothesized role of foveal inhibition (Engbert et al., 2005).

\section{Syllables as Encoding Units in a Word Copying Task \\ Abby E. Laishley ${ }^{1}$, Simon P. Liversedge ${ }^{2}$, Julie A. Kirkby ${ }^{1}$ \\ ${ }^{1}$ Bournemouth University, United Kingdom \\ 2 University of Southampton, United Kingdom alaishley@bournemouth.ac.uk}

Copying words may seem a simple task, but cognitively, there is a complex eye-mind-hand interaction in which information must be encoded and maintained in memory before written production can occur. When children copy words, they do not consistently encode words as complete linguistic units (Laishley, Liversedge, \& Kirkby, 2014). We explored whether word encoding-for-copying involves activation at the letter or syllable level. We recorded eye-movements of 7-8 year-old children as they made handwritten copies of single words presented on a wall-mounted screen. We manipulated whether words were comprised of one, two or three syllables. If syllable structure modulated word encoding, then a linear increase in encoding time with syllable structure might result. Children took longer to encode three syllable words than two and one syllable words, but needed a similar amount of time to encode two and one syllable words. This step function, rather than a linear increase, may suggest memory capacity limitations during encoding. We also examined gaze lifts during production to assess whether these were more likely to occur at syllable boundaries than elsewhere within words. Provisionally, we found no systematic evidence for such effects, suggesting a role for syllable structure during encoding, but not production during copying. 
Posters

\title{
Social influences
}

\section{Worse prediction of overt attention by physical saliency in the presence of social features}

\author{
Albert End ${ }^{1}$, Matthias Gamer ${ }^{1,2}$ \\ ${ }^{1}$ University Medical Center Hamburg-Eppendorf, Hamburg, Germany \\ 2 Julius Maximilians University of Würzburg, Germany \\ a.end@uke.de
}

According to saliency-based attention models, overt attention during free viewing of visual scenes is particularly allocated to physically salient image regions. We assumed that social features in complex naturalistic scenes would be processed preferentially irrespective of their physical saliency. Therefore, we expected saliency-based attention models to lose power in predicting gazing behavior when social information is present in the visual field. To test this hypothesis, participants freely viewed color photographs of complex naturalistic social (e.g., including faces, bodies) and non-social (e.g., including animals, objects) scenes while their eye movements were recorded. For data analysis, we calculated a saliency map for each photograph to reveal image locations that stand out from the background in terms of their physical features (i.e., color, intensity, orientation) and compared these saliency maps to participants' actual gazing behavior. We found saliency maps and fixation patterns to diverge significantly stronger for social scenes (Kullback-Leibler divergence, DKL). Consistently, the saliency-based prediction of fixation patterns was significantly worse for these stimuli (e.g., area under the ROC curve, AUC). Thus, these data demonstrate that the presence of social features weakens the influence of physical saliency on overt attention and, thereby, strongly challenge the generalizability of saliency-based attention models.

\section{GET-Social: Group Eye Tracking Environment for Social Gaze Analysis}

\author{
Ozan Deniz, Mehmetcan Fal, Ufuk Bozkurt, Cengiz Acartürk \\ Cognitive Science, Middle East Technical University, Turkey \\ ozan.deniz@metu.edu.tr
}

From the perspective of social cognition research, low-cost eye trackers provide the appropriate technology for novel methods for designing experiments on group gaze data collection, an experimental environment that has not been available so far in an affordable way. The affordability of the eye tracker sensors has also been leading to the development of accompanying software tools that support their use in various user interaction environments. We have been developing an open software tool, GET-Social, for group gaze data collection, which has a single server - multiple client architecture. The server collects synchronous gaze data from multiple client eye trackers and then displays others' gaze by animating the gaze location on client displays through a client application. The animation overlays the display of the operating system, thus allowing an application-independent use.

Besides animating gaze locations online on client displays, the server logs raw eye movement data offline and converts the data to eye movement events. The fixation locations are then used for calculating a score that aims at quantifying multiple-gaze cross-recurrence, based on Multivariate Kernel Density Estimation. We believe that GET-Social has the potential to serve both for research and application-specific developments for group eye tracking. 


\title{
When I say hello: Covert attention to real people in everyday environments
}

\author{
Kaitlin E. W. Laidlaw, Austin Rothwell, Alan Kingstone \\ University of British Columbia, Canada \\ klaidlaw@psych.ubc.ca
}

\begin{abstract}
Studies of social attention using images or videos of other people as stimuli have repeatedly demonstrated a strong bias to attend to other people, but there are many ways in which attending to a person in real life might not mirror that which is measured using computer tasks. For example, real-life strangers are looked at much less than a comparable video (Laidlaw et al., 2011). We propose that in real life, people often inhibit overt looks and instead rely on more covert methods to attend to others. To test this, we had a confederate produce two similar actions as pedestrians walked past: saying 'hey' after raising their hand and (a) waving or (b) putting a phone to their ear. Critically, these actions occurred only when pedestrians were not looking at the confederate. Results indicated that immediately after the confederate's action, pedestrians looked at the confederate more when he waved than when he spoke into the phone. These findings suggest that in real life, overt displays of social attention are sensitive to social norms, and that covert attention is frequently directed to other people in everyday life.
\end{abstract}

\section{A Dual Eyetracker Design for Measuring Shared Attention in Dynamic Oculomotor Selection}

\author{
Daniel R. Saunders, David P. Melcher, Wieske van Zoest \\ Center for Mind / Brain Sciences (CIMeC) of the University of Trento, Italy \\ daniel.saunders@unitn.it
}

Past research has suggested that the presence of a co-actor engaged in a task in the same space leads to automatic representation of their task, with a resulting influence on attention priorities. To further test this idea, we developed a novel dual eyetracker design that allowed us to record eye movement patterns simultaneously for two participants doing a visual search task, viewing a single monitor. We instructed each participant $(\mathrm{N}=26)$ to make quick, accurate saccades from a central fixation point to their target, which was a line tilted to the left for one participant and to the right for the other and located a fixed distance from the center, within an array of upright lines. In some blocks one participant searched alone, whereas in other blocks the participants searched together. Mean saccadic latency was significantly longer in the together condition than in the alone condition, which is consistent with increased interference from the co-actor's target. We saw evidence of top-down control and bottom-up capture, since shorter-latency saccades more often were directed incorrectly to the other singleton. Further work with the dual tracker methodology will help to reveal the influence of shared attention on oculomotor control. 
Posters

\title{
Effect of others' sheer presence on visuo-oculomotor tasks?
}

\author{
Denis Pelisson, Johan Ferrand-Verdejo, Eric Koun, Martine Meunier \\ CRNL (INSERM - CNRS - Univ Lyon), France \\ denis.pelisson@inserm.fr
}

The effect that the sheer presence of a conspecific has on our performance is a fundamental form of social influence, known as social facilitation. This phenomenon has been known for over a century, and concerns a wide range of behaviours from food consumption to cognition. Yet, its underlying mechanism (motivation, attention, executive control) remains debated. Here, we investigated whether visual exploration and eye movements tasks are affected by social facilitation. Subjects performed the following tasks: an oculomotor task (randomly inter-mixed pro-saccades and anti-saccades), visual search tasks (pop-out and serial) and a serial go/nogo target detection task. Two groups of 10 subjects perform these tasks either with a member of the laboratory present in front of them (presence of passive conspecific : Social Condition) or without any other person in the testing room (Alone Condition). Eye movements continuously recorded in all tasks and performance in the search tasks (reaction time) and detection task (number of hits and false alarms) will be compared between the Social and Alone groups. The serial go/nogo target detection task was first piloted in the Alone Condition to ensure an optimal performance level of $75 \%$. Data currently being collected will be presented at the meeting.

\section{Effects of gaze and facial expressions of emotions on eye movements and person judgments in social settings.}

\author{
Raphaela E. Kaisler, Manuela M. Marin, Helmut Leder \\ University of Vienna, Austria \\ raphaela.elisabeth.kaisler@univie.ac.at
}

Eye gaze plays an important role in social interactions. Previous studies showed that gazed objects increased liking ratings of the object. We asked whether this effect extends to two people shown in a natural scene. We investigated whether gaze direction and emotion of the "looking" person shown next to a person with direct gaze and neutral valence (target), would lead to higher person judgements and looking behaviour towards the target person. We manipulated emotion (happy, angry) and gaze direction (direct gaze, looking at, averted gaze) of the "looking" person and collected eye movements during a free-viewing task, followed by person judgments in a within subjects design. Faces did not differ in attractiveness. Participants were 25 male and 22 female psychology students. Our results indicated emotion effects for attractiveness and trustworthiness ratings, with increased ratings for the target face shown next to a happy face. Furthermore, we found a gaze direction effect for dwell time, referring to longer looks at the target face shown next to a direct gaze face.

Our findings suggest that eye movements and person judgements may be dissociated, and that the "being looked at" effect is not valid for natural scenes involving two people with emotional expressions. 


\title{
Friday, August $21^{\text {st }}, 1.30 \mathrm{pm}-3.30 \mathrm{pm}$
}

\section{Applications}

\section{The Effect of Expertise on Viewing of Dynamically Presented Medical Images}

\author{
Johanna K. Kaakinen ${ }^{1}$, Raymond Bertram ${ }^{1}$, Laura Helle ${ }^{1}$, Frank Bensch ${ }^{2}$, Nina Lundbom ${ }^{2}$ \\ 1 University of Turku, Finland \\ 2 University of Helsinki, Finland \\ johkaa@utu.fi
}

Eye movement characteristics of medical experts during viewing of dynamically presented medical images were examined. Early residents specializing in CT radiology ( $n=15)$, advanced residents $(n=14)$, and experienced senior radiologists $(n=12)$ viewed 24 volumes of multi-slice stack-view CT images of the abdomen. The slices were presented as a slide show with low (3 frames/second) or high speed (5 frames/second), while participants had to detect any abnormalities. The results showed that early residents were significantly poorer in detecting abnormalities than advanced residents or expert radiologists. All groups made longer fixations and shorter saccades when there was an abnormality present than when there was no abnormality. The effect of abnormalities on saccade length was of greater magnitude for expert radiologists than for early residents. Expert radiologists and advanced residents also showed greater effects of presentation speed on fixation durations than early residents. A scanpath analysis of the eye fixation patterns suggests that there was more dissimilarity among early and advanced residents than expert radiologists. The results suggest that expertise is manifested in eye movement patterns as 1) flexibility in adjusting eye movement behavior to the task demands and 2) less variability in the scanpaths across dynamic images.

\section{Scan pattern similarity in repetitive user interface examination.}

\author{
Sandra Dorothee Starke ${ }^{1}$, Natan Sorin Morar ${ }^{1}$, Chris Baber ${ }^{1}$, Neil James Cooke ${ }^{1}$, Xiuli \\ Chen $^{2}$, Andrew Howes ${ }^{2}$ \\ ${ }^{1}$ Electronic, Electrical and Systems Engineering, University of Birmingham, United Kingdom \\ ${ }^{2}$ Computer Science, University of Birmingham, United Kingdom \\ s.d.starke@bham.ac.uk
}

Sequential visual information sampling helps understand human information accumulation strategies in context of optimal decision making. This study investigated whether participants develop systematic sequential scanning behaviour when repeatedly looking at multiple information sources in a scenario resembling a semi-automated control room. Ten participants engaged in a road traffic management task. They were asked to make a series of binary decisions as accurately and as quickly as possible. In order to make the decisions, four information sources were provided via sub-windows in a user interface. These sources contained conflicting information for $75 \%$ of the overall 32 trials. Eye movements were recorded at $30 \mathrm{~Hz}$ using Tobii Glasses v.1 and mapped to the four regions of interest (ROIs). Similarity in scan patterns across trials and/or participants was calculated in Matlab using string-edit/bioinformatics methods (Levenshtein distance, sequence alignment, kmer detection) and network analysis. Results for the different analysis methods showed the general trend for scan patterns to not become more similar with elapsed trial. Scan pattern similarity also remained low when accounting for the four experimental conditions. Study results hence suggest that participants did not converge on a specific visual scanning strategy; this finding may change in the presence of feedback. 
Posters

\title{
The use of oculomotor parameters to detect hypobaric hypoxia
}

Leandro L. Di Stasi 1,2,3, Raúl Cabestrero ${ }^{4}$, Michael B. McCamy ${ }^{1}$, Francisco Ríos ${ }^{5}$, Andrés Catena $^{6}$, Pilar Quirós ${ }^{4}$, Jose A. López ${ }^{6}$, Carolina Saez ${ }^{6}$, Stephen L. Macknik ${ }^{7,1}$, Susana Martinez-Conde ${ }^{1}$

${ }^{1}$ Department of Neurobiology

Barrow Neurological Institute, Phoenix, USA

${ }^{2}$ Cognitive Ergonomics Group

Mind, Brain, and Behavior Research Center (CIMCYC), University of Granada, Granada, Spain

3 Joint Center University of Granada - Spanish Army Training and Doctrine Command, Granada, Spain

${ }^{4}$ Universidad Nacional de Educación a Distancia (UNED), Madrid, Spain

${ }^{5}$ Spanish Defence Aero-medical Center (C.I.M.A.), Madrid, Spain

${ }^{6}$ Learning, Emotion, and Decision Group

Mind, Brain, and Behavior Research Center (CIMCYC), University of Granada, Granada, Spain

${ }^{7}$ Department of Neurosurgery, Barrow Neurological Institute, Phoenix, AZ, USA distasi@ugr.es

Hypobaric hypoxia is one of the causes of air crashes. The detection of the early stages of hypoxia is therefore crucial to prevent potential aircraft accidents. Thus, an objective and early indicator of skills deterioration from hypoxia is needed to complement the subjective detection techniques currently used by pilots. Though oculomotor parameters have been proposed as one possible indicator of hypoxia, results remain inconsistent. Here, we studied the effects of short-term hypobaric hypoxia on the saccadic and intersaccadic drift velocities of Spanish Air Force aircrew. A between-groups design (short-term hypoxia group vs. control group) was used to measure changes in eye movement velocity with time-on-duty, in correlation with subjective fatigue. The results indicated a decrease in saccadic velocity for both the hypoxia group and the control group. Additionally, intersaccadic drift velocity increased in the hypoxia group only, suggesting that acute hypoxia reduces eye stability, regardless of fatigue. These results indicate that intersaccadic drift velocity could be a good candidate to be used as an objective indicator of hypoxia.

\section{Adaptive Web-Based Textbook Utilizing Gaze Data}

\author{
Mária Bieliková, Robert Moro, Jakub Simko, Jozef Tvarozek \\ Slovak University of Technology in Bratislava, Slovak Republic \\ maria.bielikova@stuba.sk
}

Web-based educational systems have become increasingly complex as students can work on assignments and at the same time interact, or read associated learning materials. Therefore, it has become hard to analyse students' interaction based on the clicks and the mouse tracking alone.

In order to get a full picture of how the students interact we have developed an infrastructure for collecting user feedback, both explicit and implicit including gaze data, which we integrated with our learning environment ALEF (used for learning programming). We use it in our UX Class equipped with 20 PCs with X2-60 Tobii eye trackers. The eye tracking data are collected in parallel from all the stations, then aggregated and analysed.

Developed infrastructure allows us to provide the system response based on the gaze events, e.g., if a student ignores provided recommendations, we can inquire why. The system itself can adapt based on the observed patterns, thus leading to more efficient learning. Moreover, by analysis of the reading patterns, we can automatically identify problematic parts of the content and recommend them for improvement. We currently test our infrastructure for the last use case providing tools for analysis of the educational content usage based on gaze data. 


\title{
An eyewear to see "yourself" by detecting movement of eyes and body
}

\author{
Shin'ichiro Kanoh ${ }^{1}$, Susumu Ichi-nohe ${ }^{2}$, Shunsuke Shioya ${ }^{2}$, Kazutaka Inoue ${ }^{2}$, Ryuta \\ Kawashima ${ }^{3}$ \\ 1 Shibaura Institute of Technology, Japan \\ 2 JIN Co., Ltd., Japan \\ 3 Tohoku University, Japan \\ kanoh@shibaura-it.ac.jp
}

We have been developing the JINS MEME, an eyewear with a capability to detect eye and body movements. The horizontal and vertical EOG (electrooculogram) signals are measured and amplified with three metal dry electrodes placed near nasion and both sides of rhinion, of which positions correspond to the bridge and nose pads of eyewear, respectively. The user's mental states like drowsiness, sleepiness, fatigue, or interest to objects can be identified by the movements and blinking of the eyes extracted from the measured EOG. And the six-axis motion sensor (three-axis accelerometer and three-axis gyroscope) mounted in the eyewear measures the body motion. As the sensor located near the head is on the body axis, this eyewear is suitable to measure user's movement or shift of center of gravity during physical exercise with a high precision. The measured signals are used to extract various events of eye and body movement by the mounted MPU chip, or can be transmitted to the external devices via Bluetooth communication. This device can enable you to look into "yourself", as well as outer scenes. In this presentation, the outline of the eyewear is introduced and some possible applications are shown.

\section{Eye Tracking in Advertising Effectiveness Research for Cultural Marketing \\ Claudia Kummer \\ Fachhochschule Burgenland $\mathrm{GmbH}$, Austria \\ claudia.kummer@fh-burgenland.at}

The aim of this paper is to illustrate the contribution of eye tracking to advertising effectiveness research in the context of cultural marketing. Two contrasting advertisement designs (A) and (B) promoting the operetta event "A Night in Venice" were tested in cooperation with an Austrian open air festival organiser. 49 students were divided randomly into two groups for the purpose of eye movement measurement. Each group was exposed to one of the stimuli, embedded in a series of various other advertisements. Subsequently, the students were interviewed in order to assess recall and recognition. Gaze paths, viewing intensity over time and top-of-mind-recall were analysed and combined with a consumer acceptance questionnaire which was completed by 242 other respondents. Analysing visual product cognition and recall, the study's results suggested only a slight advantage of subject (A), which shows a close-up view of a woman's face behind a Venetian carnival mask, over subject (B). However, when presented in direct contrast with subject (B), subject (A) achieved distinctly better entry time and dwell time averages. Practical implications are discussed a) on graphical design in advertisements and b) on how to enhance consumer insight by combining eye tracking, interview and questionnaire results. 
Posters

\title{
Fatigue in the military: Fatigue detection tests based on saccadic eye movements
}

\author{
Carolina Diaz-Piedra ${ }^{1,2,3}$, Hector Rieiro ${ }^{2,4}$, Michael B. McCamy ${ }^{5}$, Juan Suárez ${ }^{6}$, Andrés \\ Catena $^{1,2}$, Leandro L. Di Stasi ${ }^{1,2,3}$ \\ 1 Joint Center Spanish Army Training and Doctrine Command-University of Granada, Granada, Spain \\ 2 Mind, Brain, and Behavior Research Center, University of Granada, Spain \\ ${ }^{3}$ College of Nursing and Health Innovation, Arizona State University, Phoenix, AZ, USA \\ ${ }^{4}$ Department of Signal Theory and Communications, University of Vigo, Vigo, Spain \\ 5 Barrow Neurological Institute, Phoenix, Arizona, US \\ ${ }^{6}$ Spanish Air Force Helicopter School, 78th Air Base Wing, Armilla, Spain \\ dipie@ugr.es
}

Inadequate sleep and extended hours of duty are common among military personnel, leading to fatigue being recognized as a major contributing factor to operational errors. Therefore, the development of objective and sensitive fatigue detectors is critical to prevent accidents and catastrophes. In the last decades, tests based on saccadic metrics have become popular among human factors experts, with saccadic velocity as one of the most reliable indices for fatigue assessment. However, its sensitivity in the military is not yet clear, since most research connecting warfighter fatigue and saccadic velocity has been conducted in laboratory settings. Field studies remain scarce, especially in extreme conditions such as real flights.

Here, we investigated the effects of real, long flights $(\approx 120 \mathrm{~min})$ on the saccadic velocity of five newly commissioned military helicopter pilots. During the experiment, conducted during their regular training, pilots flew Sikorsky S-76C school helicopters, under instrumental flight rules in visual meteorological conditions.

Our evidence confirms the sensitivity of the fatigue test based on saccadic velocity in real flight conditions. Saccadic velocity decreased by around $5 \%$ with time-on-flight, coherent with subjective fatigue scales. This result might offer military medical departments a valid biomarker of warfighter physiological state for screening and training programs. 


\title{
The Effect of Personalized Advertisement on Visual Attention and Memory Performance
}

\author{
Kai Kaspar ${ }^{1}$, Marco Rüth ${ }^{2,3}$, Kai-Christoph Hamborg ${ }^{4}$, Moritz Köster ${ }^{4,5}$ \\ ${ }^{1}$ Social and Media Psychology, Department of Psychology, University of Cologne, Germany \\ ${ }^{2}$ Institute of Cognitive Science, University of Osnabrück, Germany \\ ${ }^{3}$ Graduate School of Neural and Behavioural Sciences, Eberhard Karls University, Tübingen, Germany \\ ${ }^{4}$ Institute of Psychology, University of Osnabrück, Germany \\ ${ }^{5}$ Center for Cognitive Science, Technical University of Kaiserslautern, Germany \\ kkaspar@uni-koeln.de
}

Personalized web advertisements have come in the focus of interest not only in research but also in the public debate. However, our knowledge about effects of personalization on attention and memory are surprisingly limited. In an eye-tracking study, participants performed an information search task on web pages of an online news portal with either personalized or non-personalized versions of banner ads. We found that ads were generally rarely fixated, indicating strong banner blindness. Nonetheless, personalization increased attention towards ads significantly. Moreover, personalization substantially enhanced recognition for ad content. In contrast, the overall exploration of web pages quantified by a progressive entropy measure and recognition performance of task-relevant information were not affected. An in-depth analysis of eye movements on banner ads revealed a relatively stable spatiotemporal course of gaze behavior across regions of interest. Visual exploration of ads typically proceeded from ad pictures to the logo and finally to the banner ad slogan. We may conclude that a small effect of ad personalization on attention is paralleled by substantial effect on memory performance.

Köster, M., Rüth, M., Hamborg, K. C., \& Kaspar, K. (2014). Effects of Personalized Banner Ads on Visual Attention and Recognition Memory. Applied Cognitive Psychology. DOI:10.1002/acp.3080

\section{Aggregation of Driver Distraction for Large Scale Real-World Studies}

\author{
Lucas Paletta, Roland Perko, Michael Schwarz \\ JOANNEUM RESEARCH Forschungsgesellschaft mbH, Austria \\ lucas. paletta@joanneum.at
}

Eye tracking research about driver distraction, applied driving tasks in real world traffic environments (Harbluk et al., 2002, Young \& Mahfoud, 2007), has so far been demanding massive manual intervention, for the annotation of extensive duration of head camera videos (Chattington et al., 2009; Zhang \& Peterson, 2011). We present a novel methodology that enables the automated localisation of the driver's gaze within a visual ROI (region-of-interest) and its environment, from various approaches of drivers who are equipped with eye tracking glasses. Gaze allocations in the driver's environment are tracked within the video with computationally efficient optical flow methodology (Zach et al., 2007) and finally projected onto selected key frames for aggregated eye movement analysis, being visualized in terms of gaze distributions and heat maps. This novel technology for analysis and visualization enables for the first time a fully automated estimation of the distraction from roadside ROIs for large scale experiments. The results of a sample study with 12 drivers including automated analysis of more than 100.000 video frames depict driver distraction before and after billboard construction, including automated generation of statistics on fixations, dwell time and number of looks within the ROIs. 
Posters

\title{
A gaze-contingent paradigm as a basis for interactive training of the phonetic contrasts discrimination: a pilot study
}

\author{
Joanna Dreszer ${ }^{1,2}$, Bibianna Balaj ${ }^{1,2}$, Jacek Matulewski ${ }^{2,4}$, Monika Lewandowska ${ }^{2,3}$, tukasz \\ Goraczewski ${ }^{2}$, Włodzisław Duch ${ }^{2,5}$ \\ 1 Institute of Philosophy, Nicolaus Copernicus University, Torun, Poland \\ 2 Centre for Modern Interdisciplinary Technologies, Nicolaus Copernicus University, Torun, Poland \\ 3 World Hearing Center, Institute of Physiology and Pathology of Hearing, Warsaw/Kajetany, Poland \\ 4 Institute of Physics, Nicolaus Copernicus University, Torun, Poland \\ ${ }^{5}$ Department of Informatics, Nicolaus Copernicus University, Torun, Poland \\ joanna.dreszer@gmail.com
}

A discovery that one's own actions induce interesting responses of the environment may encourage exploratory behavior, curiosity, and increase motivation. Furthermore, it has been found that social interaction with another person affects speech learning in infants (Kuhl 2004). It is not clear, however, whether an interaction without a social component (e.g. with a toy or a movie) also improves such process.

The goal of the present study was to design and test an interactive movie for 8-10-monthold infants. The movie was developed as a part of ongoing research project on language learning in infants and young children. We expect that training of the phonetic contrasts discrimination in active way will be more effective than passive listening of speech sounds. Six healthy infants aged between 7 and 10 months watched an interactive movie. The eye tracking technology was applied. A newly-developed gaze-contingent paradigm (Wang et al. 2012) as a basis for interactive movie construction was introduced to allow infants to control their environment and induce rewarding responses of the movie's characters.

The interactive movie may be served as an experimental condition with minimal degree of social interaction. It might be a new approach for training clinical populations, e.g. children with autism.

\section{SMOOVS: A smooth-pursuit based text entry system Otto Hans-Martin Lutz, Antje Christine Venjakob, Stefan Ruff \\ Chair of Human-Machine-Systems, Technische Universität Berlin, Germany lutz@tu-berlin.de}

Several concepts have been developed to exploit smooth-pursuit eye movements in gaze interaction at public displays. Participants usually select one of 6-12 moving elements by following it with their gaze. The approach has proved successful, but it is unclear if it can be transferred to a more complex scenario, as e.g. in the implementation of a speller that displays the entire alphabet plus a number of special characters. To test this concept, the socalled Hex-O-Spell, originally used with EEG, was adapted to the context of pursuit-based gaze selection. Letters and characters were grouped in chunks of six which moved away from the center of the screen. To select a particular letter, its chunk is followed with the eyes. After the initial movement, the letters of the selected chunk move away from one another, following the letter of choice leads to its selection. To gain insight into an optimal speed of movement, a user study with 24 participants tested four different speed levels. At $300 \mathrm{px} / \mathrm{sec}$ the quickest and overall least error-prone interaction was realized. $300 \mathrm{px} / \mathrm{sec}$ was also perceived to be most comfortable. Usability features, such as feedback, visual and interaction design will be discussed with the postersession's audience. 


\title{
Can flight phase be inferred using eye movements? Evidences from real flight conditions
}

\author{
Sébastien Scannella, Vsevolod Peysakhovich, Florian Ehrig, Frédéric Dehais \\ Superior Institute of Aeronautics and Space - ISAE, France \\ s.scannella@isae.fr
}

Operating an aircraft is a cognitively challenging task in which pilots have to continuously monitor flight deck instruments. The present study attempts to find out whether eye movements are indicative of different monitoring activities within the flight phases. Eleven pilots, equipped with a head-mounted eye tracker on board of a Robin DR-400 light aircraft, performed traffic patterns that consisted of three consequent flight phases - take-off, downwind and landing. As shown by statistical analysis over saccadic rate and visual entropy, all three flight phases induced significantly different ocular behaviors. The eye metrics values were higher during downwind phase, indicative of an active visual search to identify visual references on the landscape and to configure the airplane (flaps, engine). The saccadic rate and visual entropy were lower during take-off and minimal during landing phases, as these phases are the most demanding with a visual focus on a small set of instruments. Most importantly, using linear discriminant analysis, we found that the combination of the two metrics can separate the three flight phases across pilots with an accuracy of $88 \%$. These findings are promising for the on-board eye tracking and characterization of pilots' ocular behavior in real flight conditions.

\section{Eye-gaze Based Attention Estimation in Minimal Invasive Surgery \\ Georgios Pipelidis, Michael Matscheko, Alois Ferscha \\ Johannes Kepler Universität Linz, Austria \\ pipelidisg@gmail.com}

We hypothesize that laparoscopic surgical suboptimal outcomes in patient safety measures are correlated with (i) cognitive load / level of attention of the operating surgeon, (ii) the frequency and degree of disruptions to the surgical workflow, and (iii) the misalignment of visual and motor axes in laparoscopic equipment / setting (eye-hand coordination). In this paper we present an eye tracking approach to assess the surgeons attention based on a clinical study with 6 laparoscopic surgeries. At the eye-gaze level an efficient way for filtering saccades over tremors is presented, and a strategy for reducing the complexity of data by methodical discretizing fixations over saccades while a preserving maximum of the semantic information is presented. This procedure was automated and videos were generated to estimate the surgeons attention levels and to identify possible causes of distraction. 


\title{
Linking the ability in cognitive tasks to efficient gaze allocation during driving
}

\author{
Andrew Kerr Mackenzie, Julie M. Harris \\ University of St Andrews, United Kingdom \\ akm9@st-andrews.ac.uk
}

In driving, the misallocation of visual attention has been suggested as a major contributing factor to vehicle accidents. One possible reason for this misallocation of attention is that the relatively large cognitive demands of driving limits the ability to efficiently allocate gaze. Knowing more about how these cognitive demand characteristics influence eye movement behaviour may contribute to the development of better driver training or assessment programmes. We present an experiment which aims to identify potential relationships between individuals' cognitive ability and their visual performance when driving. Drivers performed a battery of cognitive tasks (multiple object tracking and object avoidance tasks) targeting aspects of cognition such as sustained attention, dual-tasking, covert attention and visuomotor skill. They also drove a number of courses in a driving simulator. Eye movements were recorded throughout. We found that individuals who performed better in the cognitive tasks exhibited more efficient eye movement strategies when driving, e.g. increased road scanning, larger and faster saccades and more efficient use of the mirrors. These results suggest a direct link between an individual's cognitive ability and their capacity for exhibiting useful visual behaviour when driving. We argue therefore, that training in cognitive tasks may improve visual performance in driving.

\section{Para-sight: A visual search study into the effects of product similarity on brand location and recognition.}

\author{
Timothy Paul Holmes ${ }^{1,2}$, Haywood Crispin ${ }^{3}$, Alice Georgina Lowenhoff ${ }^{1}$ \\ 1 Acuity Intellgence Ltd, United Kingdom \\ 2 Royal Holloway, University of London, United Kingdom \\ 3 SSL Research, United Kingdom \\ tim@acuity-intelligence.com
}

Imitation is sometimes described as the sincerest form of flattery, but in business, as in nature, it can be used as a strategy to undermine a dominant entity by gaining access to a limited resource whilst remaining undetected. Copycat brands use design elements from a leading brand to gain market share by association, but are they always detected by shoppers? We used a visual search paradigm to test 8 well known UK (target) brands alongside read copycat (distractor) brands on photo-realistic life-size images of supermarket shelves to determine the effects on location (eye-movements) and decision making when one, both or neither were present. We also varied the spatial resolution and saturation to explore the contribution of these at each stage. 28 participants each saw 64 shelves for up to 10 seconds. Colour dominated location accuracy whilst higher spatial resolution was needed for correct decision making. Participants located the copycat brands $740 \mathrm{~ms}$ faster than the leading brands when both were present, and incorrectly identified distracters as the target $25 \%$ of the time, rising to $75 \%$ when just the distracter was shown. We discuss the implications for both normal and visually impaired shoppers in the real-world. 


\title{
Attentional bias towards preferred brands in smokers - dot-probe study
}

\author{
Ewa Domaradzka1 ${ }^{1}$, Maksymilian Bielecki ${ }^{2}$ \\ 1 Institute of Psychology of the Polish Academy of Sciences \\ 2 University of Social Sciences and Humanities, Poland \\ edomaradzka@psych.pan.pl
}

Purpose: Preferential processing of stimuli related to addiction is a well validated phenomenon. In our study we tested if this effect is specific enough to differentiate participants' reactions to the favorite cigarette brand when compared with other cigarette brands' pictures. Methods: 42 nicotine addicts were tested using dot-probe procedure. Standardized photos of cigarette packages were used as stimuli. Data were collected using 2k Eye-link tracker. Results: Significant differences were observed in trials contrasting preferred with non-preferred brands. Latency to initiate the saccade and time needed to reach the dot were longer, and peak velocity was lower when participants had to disengage from their preferred brand picture. They were also faster in reaching the position occupied previously by their favorite brand when compared with other conditions. No such effects were observed during the first fixations following picture presentation at the beginning of the trial. Conclusions: Attentional bias typical for addicts is more pronounced when favorite brand is presented (when contrasted with other products from the same category). This effect seems to be limited to later stages of stimulus processing, as suggested by lack of favorite-brand preference in first saccade latency and direction.

\section{Eye movements, aesthetic preference and drawing Eugene McSorley, Louis Williams, Rachel McCloy \\ University of Reading, United Kingdom e.mcsorley@reading.ac.uk}

Forming an aesthetic preference is a continuous process and examining the pattern of eye movements made when forming an aesthetic judgement can be used to uncover this process. Using this approach it has been found that people spend longer looking at stimuli they aesthetically prefer and make more fixations. It remains unclear to what extent there is a relationship between the aesthetic judgement and the activity which leads to its production. Here we examine whether this pattern is also found when making judgements about drawing preference. Aesthetic ratings were taken for geometric shapes (either before or after the main experiment). These were presented two at a time and participants were asked to either free-view them or make a drawing preference between them. Each trial was then subsequently classified on the basis of aesthetic and drawing preference. It was found that participants looked more and for longer at the shape they aesthetically prefer and that they prefer to draw suggesting that there are common processes involved in aesthetic judgement and production (i.e., in this case drawing). A second experiment examined the preferences of experts and found a similar pattern of results suggesting that expertise does not change this relationship. 
Posters

\title{
Food choice and eye movements: The eyes have it on the day - not so much on the next.
}

\author{
Andreas Jarvstad, Peter J. Rogers, Jeffrey M. Brunstrom, lain D. Gilchrist \\ University of Bristol, United Kingdom \\ a.jarvstad@bristol.ac.uk
}

Fixation and decisions are positively associated - looking increases the probability of choosing. We explored this association in the context of food choice. Participants made choices between pair-wise presented meals varying in type and portion-size. The display was saccadecontingent, with un-fixated foods occluded, controlling for low-level visual drivers of fixation. To separate out, and model, stable from more transient drivers of choice, we tested participants in two separate sessions (1-week-apart). The stimuli were identical across session 1-2, but were presented in different random orders. To predict food choice, we fit logistic regression models. For Session1, models based on fixation behaviour and food rating preferences fit choice data equally well. A model including both fixation and rating data performed best $(<\mathrm{AIC})$, showing that each contributes independently to choice. We then asked how well food ratings and fixation behaviour in Session1 predicts choice in Session2. When this 'prediction' is compared to the previous 'fitting', fixation-based models show a $20 \%$ drop in performance, whereas ratings-based models drop at worst $5 \%$. Thus, subjective ratings and food properties (e.g., calories) capture stable choice preferences. However, the association between looking and choice appears largely driven by transient and momentary factors rather than stable preferences over time.

\section{The interplay of low-level and higher-level dimensions in abstract and representational artworks. \\ Martina Jakesch, Helmut Leder \\ University of Vienna, Austria \\ martina.jakesch@univie.ac.at}

Literature suggests that low-level and higher-level dimensions guide our gaze when viewing images. Eye-tracking methods therefore can be a useful tool to uncover the appeal of art. Here, we focus on this interplay by using visually salient Areas of Interest (SAL) and Areas of Interest representing higher-level features (SUB) in combination with two classes of art (abstract versus representational). In a free-viewing task, 30 pictures were presented 60 s each. Areas of Interest were produced by using the Saliency Toolbox (Walther \& Koch, 2006; SAL) or by averaging overlapping areas of 8 independent raters (SUB). Area sizes did not differ significantly between abstract and representational pictures. In line with our expectations, we found significant more fixations in SAL AOIs in abstract paintings and vice versa, significantly more in SUB AOIs in the condition representational. SUB and SAL AOIs overlap to a significant higher percentage in abstract pictures indicating a stronger congruence between computer and humans. People seem to fixate longer in SAL AOIs when the content is abstract than representational. Time bins will be used to test the dynamics of SAL and SUB over time in a final step. Results will be discussed in respect to models of art perception. 


\title{
Tracking eye movement in medical ultrasound video clip analysis of horse legs
}

\author{
Shelley Imanuela Buchinger ${ }^{1}$, Marcus Kaar ${ }^{2}$, Theresia Licka ${ }^{3}$, Christian Valuch ${ }^{1}$, Ulrich \\ Ansorge 4 \\ ${ }^{1}$ Cognitive Science Research Platform, University of Vienna, Austria \\ ${ }^{2}$ Center for Medical Physics and Biomedical Engineering, Medical University of Vienna, Austria \\ ${ }^{3}$ Clinical Unit Horse Surgery, University of Veterinary Medicine, Austria \\ ${ }^{4}$ Faculty of Psychology, University of Vienna, Austria \\ shelley.buchinger@univie.ac.at
}

Healthiness of horse tendons is frequently analyzed by the means of Ultrasound videos. Unfortunately, these videos often contain artefacts caused by air what complicates the task of making a correct diagnosis. On one hand, artefacts might be easily confounded with existing lesions leading to false positives and on the other hand, they might cover existing lesions leading to false negatives. In the frame of the grant CS 11-09 from the WWTF we developed the AirTest tool that detects artefacts caused by air automatically. Potentially disturbed regions are indicated by the means of white arrows on the top of each movie picture. In the frame of a mixed experiment combining eye tracking with other input devices, we try to find an answer to the following questions: (1) Do the indicators of potentially disturbed regions help the viewer to improve the correctness of the diagnosis? (2) How do the viewers search for lesions in frames containing more and others less artefacts? (3) Which is the impact of the arrows indicating artefacts on the eye movement? In this poster we would like to present the setup and first results of our work in progress.

\section{Scene viewing and gaze analysis during phonetic segmentation tasks}

Arif Khan ${ }^{1,2,3}$, Ingmar Steiner ${ }^{1,2}$, Ross G. Macdonald ${ }^{1}$, Yusuke Sugano ${ }^{1,4}$, Andreas

\author{
Bulling ${ }^{1,4}$ \\ ${ }^{1}$ Saarland University, Germany \\ 2 DFKI GmbH, Saarbrücken, Germany \\ ${ }^{3}$ Saarbrücken Graduate School of Computer Science, Germany \\ ${ }^{4}$ Max-Planck-Institute for Informatics, Saarbrücken, Germany \\ arifkhan@coli.uni-saarland.de
}

Phonetic segmentation is the process of splitting speech into individual sounds. Human experts perform this task manually by analyzing auditory and visual cues using analysis software, but one minute of speech can take an hour to segment. To improve automatic segmentation, which cannot yet match human experts' accuracy, we analyzed the behavior of experts performing segmentation tasks, using a stationary eye-tracker.

A 46s recording of "The Northwind and the Sun" was segmented using standard phonetic software (Praat, http://praat.org). Gaze activity was captured using a Tobii TX300. The computer screen and user interaction were recorded as well. Data collection is ongoing, and we plan to record 12 experts.

During the task, experts zoom in to view short spans of audio; we analyzed the scene viewing behavior (fixation locations and durations), as well as the audio segments to which they listen. Moreover, activity over the entire task was analyzed within and across participants. Preliminary results provide new insight about experts' behavior during phonetic segmentation tasks. Identifying critical features of visible speech in this manner will allow us to model their importance for automatic segmentation. It also exposes behavioral differences across individual experts performing the same task. 
Posters

\title{
Eyes movement in the visual semantic search
}

\author{
Irina Blinnikova, Anna Izmalkova, Natalia Rotova \\ Moscow State Lomonosov University, Russian Federation \\ blinnikovamslu@hotmail.com
}

This study analyzed oculomotor scanning strategies of letter matrices space in the process of finding semantically meaningful units using SMI equipment and software. Tables, consisting of 225 cells (15x15), filled with letters were utilized as stimulus material. Among these letters 10 words were located in a random order either horizontally or vertically. The subjects $(n=28)$ had to identify and name them within $40 \mathrm{~s}$ time interval. The number of correctly named words, errors, and basic indicators of eye movement was registered. The spatial organization of semantic search was revealed. First, the specific strategies of eye movement were revealed, which are, so to say gravitate to target stimuli; in the areas of location of test words the total duration of fixations was significantly higher than in other areas of the matrix; in these zones significantly higher number of return saccades were recorded. Second, it was found that although the density of semantic units was approximately the same in all the tables' space, subjects found the words by clusters: the average distance between the detected words was significantly smaller than between two randomly selected test units in the table. Third, horizontally placed words were detected much more often than vertically placed ones. 


\title{
Biological influences
}

\author{
The phase of menstrual cycle modulates eye movements in \\ evolutionary-relevant context. \\ Joanna Pilarczyk, Emilia Schwertner, Michat Kuniecki \\ Jagiellonian Univeristy, Poland \\ joanna.pilarczyk@uj.edu.pl
}

Due to the variations in hormone levels across the menstrual cycle, during the pre-ovulatory (follicular) phase women are more sensitive to reproductively-relevant stimuli, while in the post-ovulatory (luteal) phase they are sensitive to stimuli related to risk of pregnancy termination. The engagement of attention by evolutionary-relevant stimuli measured using eye-tracking should reflect changes in this sensitivity.

Female participants $(\mathrm{N}=20)$ were tested twice, first in the luteal and then in the follicular phase, or vice-versa. Progesterone level was measured from saliva sample. One hundred and twenty images from six evolutionary-relevant categories were presented: Children, High-calory food, Low-calory food, Threat, Disgusting objects, and Erotic scenes. Images were segmented to key region and background. Number of fixations in the key region were compared in the two menstrual phases.

In the luteal phase first fixation fell more often in the key regions of Children $(t(19)=2.4, p=$ $.026)$ and Threat images $(t(19)=3.0, p=.007)$ than in the follicular phase. This tendency was sustained during following fixations in case of Threat; $t(19)=2.2, p=.042$. The results show that the phase of menstrual cycle influenced both capture, as well as hold of attention.

\section{Effects of sex and sexual orientation on the visual exploration of facial attractiveness

\author{
Aleksandra Mitrovic ${ }^{1}$, Pablo Tinio ${ }^{2}$, Helmut Leder ${ }^{1}$ \\ 1 University of Vienna, Faculty of Psychology, Austria \\ ${ }^{2}$ Queens College of the City University of New York, Division of Education, New York, U.S.A. \\ aleksandra.mitrovic@univie.ac.at
}

The finding that attractive faces attract longer looks is often interpreted in terms of evolutionary adaptations favoring reproductive success. We challenged this notion by comparing gaze behavior of heterosexual and homosexual individuals looking at male and female faces. We tested whether gaze behavior of individuals reflects either their sexual orientation or their motives for reproduction. Real world scenes were presented depicting two persons of the same sex, one being distinctly more attractive than the other. We recorded eye movements while 40 participants (half heterosexual, half homosexual; half female, half male) freely viewed the scenes. In a second block, participants rated the attractiveness of all faces. For male subjects, the pattern of fixation number and duration and attractiveness ratings were in line with their sexual orientation. Interestingly, women's gaze behavior reflected an increased attention for male faces, whereas their attractiveness ratings were higher for female faces. Additionally, less attractive faces were looked at shorter and rated lower, when the face's sex was in line with participants' sexual orientation. These findings suggest that the influence of sexual orientation and motives for reproduction on gaze behavior cannot be disentangled completely. 
Posters

\title{
Influences of individual differences
}

\section{Eye movements and executive control in older adults: Age-related decrease in response inhibition.}

\author{
Thomas Lüttke, Anna Lena Kahl, Christian Vorstius, Ralph Radach \\ Bergische Universität Wuppertal, Germany \\ thomas.luettke@uni-wuppertal.de
}

Two experiments were conducted to study the influence of working memory (goal neglect) and task difficulty (response selection) on oculomotor response inhibition for 22 young participants (23.3 years) and 21 aged participants (68.2 years). First, a delayed oculomotor response task was used to examine the level of automated response suppression with and without additional load on working memory. Second, a number-cued saccade task with attention capture served to determine the influence of task difficulty on the ability to suppress the effect of irrelevant visual stimulation on the planning and execution of goal directed saccades.

In both tasks older participants showed a substantially inferior level of response inhibition compared to their younger counterparts. This was indicated by a much higher frequency of premature and/or misdirected saccades, and, as a consequence, more frequent corrective movements. These differences were pronounced in the delayed oculomotor response task, especially when task difficulty was high. While a demand for more complex response selection amplified the age-related performance deficit, increased working memory load did not appear to have a significant effect. Our results add a new angle to the ongoing debate on the contribution of deficits in response suppression to age-related decline in general cognitive performance.

\section{Do video-game players have a larger spotlight of attention compared to non-players?}

\author{
Galina Henz, David Mack, Uwe Ilg \\ Hertie Institute for Clinical Brain Research, Germany \\ galina.henz@student.uni-tuebingen.de
}

We reported that video-game players (VGP) have shorter saccadic latencies and higher peak velocities compared to non-players (Mack \& Ilg 2014). These benefits did not result from an increased error rate. To find a possible explanation of the VGP benefits, one might speculate that the attentional spotlight of VGPs might be larger as the spotlight of non-players. In order to test this hypothesis, we developed a dual task paradigm in which the subjects had to report the orientation of the fixation target and simultaneously report the number of visual targets ( 0 to 7 ) arranged at four different eccentricities ( 2 to 11 degrees). We tested 19 VGPs (4 hrs weekly gaming time) and 27 age-matched non-players, exclusively males.

Our data clearly show that VGPs had higher performance in the enumeration task, independent of eccentricity. We also performed a control experiment in which our subjects were asked to determine which of two stimuli contained the larger numerosity. Here, we did not find a significant difference in performance. The results of both experiments indicate that VGPs do not simply have a superior number sense. The lacking interaction between eccentricity and enumeration performance refutes our hypothesis related to an increased spotlight of attention. 


\title{
Personality traits and eye movements: an eye-tracking and pupillometry study
}

\author{
Anne-Kathrin Wilbers ${ }^{1}$, Alina Vennekötter ${ }^{2}$, Moritz Köster ${ }^{2,3}$, Kai-Christoph Hamborg ${ }^{2}$, \\ Kai Kaspar ${ }^{1}$ \\ ${ }^{1}$ Social and Media Psychology, Department of Psychology, University of Cologne, Germany \\ 2 Institute of Psychology, University of Osnabrück, Germany \\ ${ }^{3}$ Center for Cognitive Science, Technical University of Kaiserslautern, Germany \\ a.wilbers@uni-koeln.de
}

Top-down influences on attention control have come in the focus of interest. However, while effects of the current task on eye movements have been extensively studied, timeindependent personality traits have been widely neglected so far.

However, some recent studies showed that inter-individual differences in personality traits may be linked to inter-individual differences in gaze behavior. In an ongoing study, we scrutinize this relationship and investigate whether persons differing in personality can be distinguished on the basis of eye movement signatures. For this purpose, a new set of visual stimuli was created that incorporates several regions of interest differing in color, gist, and valence. Eye movements and pupil diameter are captured by an eye-tracker during image observation. Initial results indicate that the individual extent of extraversion negatively correlates with the duration of fixations independently of stimulus type. Moreover, a higher degree of neuroticism leads to a stronger avoidance of peripherally presented negative stimuli when centrally presented fearful faces are displayed at the same time. On the poster, we will also show effects on pupil diameter.

This work is supported by an Uoc Postdoc Grant of the University of Cologne and the Excellence Initiative of the German Research Foundation (KK).

\section{Charlotte Mezö, David Mack, Uwe Ilg \\ Hertie Institute for Clinical Brain Research, Germany \\ c.mezoe@student.uni-tuebingen.de}

Eye movements of chess players and novices in different settings

We explored the influence of expertise in the domain chess on eye movements and estimation of different chess problems. We recruited 17 chess players with identified German Evaluation Number and 31 novices. In our first experiment, we presented six different chess problems, each for five seconds, and recorded the eye movements of our subjects (Arrington) while they selected the best move to checkmate the black king. Chess players had a higher percentage of correct answers. Interestingly, our eye movement recordings showed that chess players performed less and smaller saccades than novices. As a consequence, they had a smaller viewed area compared to novices. We examined the effects of the factor chess scene and chess experience on the viewed area by means of a 2-factorial ANOVA, both factors were highly significant.

To test for general differences, we asked our subjects to perform anti-saccades as well as smooth pursuit. Interestingly, we only found age-related differences here, but no differences in the eye movement parameters due to chess experience.

Our findings demonstrate that chess players restrict their viewed area compared to novices, indicating an expertise-based optimization of their oculomotor behavior. However, we did not observe a spillover of the effects to different oculomotor tasks. 
Posters

\section{The study of vestibular dysfunctions in athletes using eye tracking technology

\author{
Oxana Klimova, Artem Kovalev \\ Lomonosov Moscow State University, Russian Federation
} \\ OKli07@yandex.ru}

Integration of sensory information provided by vestibular, visual and proprioceptive channels play a significant role in sports. The present study is aimed at developing a method of the evaluation of vestibular disturbances in sportsmen using virtual reality technology and eye tracking. To achieve this goal the vestibular dysfunctions were initiated during the vection illusion perception. The vection illusion was evoked by the virtual environment rotation in the CAVE virtual reality system. Four groups were tested - professional figure skaters, professional football players, professional wushu fighters and people who had very little experience in sports or had no such experience at all. 110 subjects took part in the experiment. The quantitative evaluation of vestibular dysfunction was based on eye movement characteristics which were registered during the perception of the vection illusion. Results showed that there were significant differences in eye movement characteristics for professional figure skaters and other 3 groups. The professional figure skaters showed the most active eye movements - the increase in blinks and fixations frequency, the decrease of their durations and saccadic amplitudes. Other sportsmen showed significantly lower eye movement activity compared to skaters and higher activity compared to native subjects.

\section{Gaze behavior during obstacle crossing in children and young adults Anna-Maria Gorgas ${ }^{1}$, Kate Hamel $^{2}$, David Anderson ${ }^{2}$ \\ ${ }^{1}$ St. Poelten University of Applied Sciences, Austria \\ 2 San Francisco State University, California, USA anna-maria.gorgas@fhstp.ac.at}

The purpose of this study was to examine gaze patterns in children when crossing obstacles of different heights. By comparing data from children and young adults, this study highlighted age-related differences in how vision is utilized during locomotion.

Five children ( $\mathrm{CH}, 7.7 \pm 3.4 \mathrm{yrs}$ ) and nine adults (YA, 26.8 $\pm 3.4 \mathrm{yrs})$ completed the testing protocol. Participants walked along a $10 \mathrm{~m}$ walkway and crossed obstacles situated in their walking path under different conditions. Gaze behaviors were recorded using a headmounted eye-tracking system (ASL).

Children showed significantly longer normalized gaze fixation durations in the foot placement regions prior to the obstacle than adults (YA: $0.6 \pm 1.6 \%, \mathrm{CH}: 3.4 \pm 4.6 \%$ of travel time). Conversely, YA fixated significantly longer in the area after the obstacle (YA: $11.5 \pm 8.2 \%$, $\mathrm{CH}: 4.3 \pm 3.2 \%$ of travel time). Latency times (gaze shifting away from the foot placement area to foot contact in this area) were shorter in $\mathrm{CH}$ compared to YA (first step after obstacle, YA: $1676 \pm 990 \mathrm{~ms}, \mathrm{CH}: 824 \pm 940 \mathrm{~ms}$; second step after obstacle, YA: $1696 \pm 1008 \mathrm{~ms} ; \mathrm{CH}$ : $924 \pm 1115 \mathrm{~ms})$.

These findings suggest that $\mathrm{CH}$ adopted a shorter feedforward control strategy for obstacle negotiation than YA indicating a still ongoing development of sensory control processes. The link between gaze and attentional demands on dynamic stability should be further investigated. 


\title{
Manual versus Saccadic Assessment of Inhibition and Switching Deficits: Evidence the Saccadic System Exhibits Resistance to Aging \\ Liana Machado, Chris Brett \\ University of Otago, New Zealand \\ liana@psy.otago.ac.nz
}

Inhibition and switching deficits occur with healthy aging and many clinical conditions, compromising performance of daily tasks and thus quality of life. Saccade tasks have often been used to assess inhibition and switching deficits, but they require access to expensive eye-tracking equipment and trained personnel. Moreover, with respect to switching, the aging literature suggests manual tasks may be more sensitive to deficits than saccade tasks. The current research directly compared the two response modalities for assessing inhibition and switching abilities, and characterized age-related changes. We measured in 60 young adults (18-24 years) and 60 older adults (60-72 years) performance on saccade and key press tasks identical in all ways except for response modality. Results showed that saccadic and manual responses significantly correlated, suggesting key press tasks can act as a proxy for saccade tasks. Regarding aging effects, compared to saccadic responses, manual responses showed more robust inhibition and switching deficits, which suggests key press tasks are more sensitive to age-related decline. Moreover, a lack of switching deficits for saccadic responses suggests the oculomotor system may be more resistant to age-related decline. Future research is needed to test whether these patterns extend to clinical populations with inhibition and switching deficits.

\section{Cultural Differences in Visual Attention}

\author{
Albandari Sultan Alotaibi, Geoffrey Underwood, Alastair D. Smith \\ University of Nottingham, United Kingdom \\ Ipxaa@exmail.nottingham.ac.uk
}

Do individuals from different cultures search scenes differently? It is proposed that the mechanism that differentiates individuals from different cultures in the field of visual attention is their tendency to adopt either an analytic or a holistic thinking style. In the current experiment, we investigate this question-using eye tracking technology. The number of fixations was recorded for 30 participants (15 British students and 15 Saudi students) while they viewed 60 real-world photographs. The Saudi participants showed a greater number of fixations and longer reaction times compared to the British group. This may indicate that analytic cognitive processing provides greater capacity for controlled attention, giving the advantage of greater capability to direct attention towards task's goals. The question of how culture affects searching strategies, and which particular cultural factors are in play, is still open for future study. If the role of culture becomes well understood, it could have a noticeable effect on modelling eye movement behaviour. 
Posters

\title{
The influence of motor experience in the visual behavior during observation of motor activity known and not known \\ Olival Cardoso do Lago ${ }^{1,2}$, Paulo Schor ${ }^{2}$ \\ ${ }^{1}$ Biomechanics Lab - Faculty of Physical Education ESEF-Jundiaí, Brazil \\ 2 Ocular Bioengineering Lab - Dept. Ophthalmology - Federal University of São Paulo, Brazil olago@esef.br
}

Daily human and sports demands a preponderance of visually guided actions. The sports performance depends not only on the implementation of accurate motor action but also effective visual information so that the athlete can e.g., determines the trajectory of the ball and the time to intercept it. Does the place at which people look while they evaluate certain scene depend exclusively on the task and/or some oculomotor behavior influenced by the motor experience in similar scenes? Using the sports as a paradigm, we are investigating whether the systematic training leads to a transfer of eye movement patterns between similar motor activities. In the present study, we examined the pattern of fixation and eye movements when athletes watching motor activities of sport that practice and similar modalities that do not practice. In the experiments, were tracked the eye movements of three groups of athletes $(n=30)$ experienced: Tennis Players, Goalkeeper Football and Handball, while watched videos with motor activities (service, penalty and 7 meters) of their respective sport and other modalities studied. The results are discussed in terms of fixations, saccades and scanpath, and if they feature oculomotor behavior that could be used as evidence of the influence of systematic training.

\section{Eye movements as an indicator of spatial disorientation in pilots and amateurs}

\author{
Bibianna Balaj ${ }^{1,2}$, Piotr Francuz ${ }^{3}$, Agnieszka Fudali-Czyż ${ }^{3}$, Paweł Augustynowicz ${ }^{4}$, Paweł \\ Stróżak ${ }^{3}$, Rafał Lewkowicz ${ }^{5}$, Olaf Truszczyński ${ }^{5}$ \\ ${ }^{1}$ Institute of Philosophy, Nicolaus Copernicus University in Torun, Poland \\ ${ }^{2}$ Centre for Modern Interdisciplinary Technologies, Nicolaus Copernicus University in Torun, Poland \\ ${ }^{3}$ Institute of Psychology, The John Paul II Catholic University of Lublin, Poland \\ ${ }^{4}$ Faculty of Economics, Maria Curie Skłodowska University in Lublin, Poland \\ ${ }^{5}$ Military Institute of Aviation Medicine in Warsaw, Poland \\ bibianna.balaj@gmail.com
}

Main goal of our research was to search for eye movement indices, which can be monitored during flight profiles posing threat of spatial disorientation. We were looking for differences between experts (pilots) and amateurs (non-pilots) in the performance of the flight task especially for spatial disorientation originating from vestibular system. Expertise is frequently taken into account when examining pilots' eye movements, but not in the context of spatial disorientation.

The experiment was carried out in the Military Institute of Aviation Medicine in Warsaw. The subjects (15 pilots; 15 non-pilots) have undergone a series of tasks in the flight simulator GYRO-IPT regarding piloting the aircraft during flight profiles containing selected situations related to the risk of the emergence of spatial disorientation (eg. slope of the runway, false horizon, somatogyral illusion, leans illusion). During performation of flights eye movements were recorded.

Comparison of eye movements in experts and novices allowed us for designing a cognitive model of visual scanning during flight tasks. Expert pilots in comparison to amateurs had shorter fixation time and higher frequency of fixations. Experts more effectively collect visual information, which leaves them more resources to monitor less critical tasks and cope with changing requirements of a task. 


\title{
Post-Editing Effort as a Measure to evaluate Quality of Machine Translation Jean Nitzke \\ Univeristy of Mainz, Germany nitzke@uni-mainz.de
}

Technical development and globalization continue to raise the need for translations. To improve efficiency and cost-effectiveness, organisations increasingly make use of machine translation (MT) and edit the MT output to create a fluent text that adheres to the given text conventions. This procedure is known as post-editing (PE).

In a series of experiments, 24 translators (twelve professionals and twelve semiprofessionals) produced translations from scratch, post-edited and monolingually postedited MT output. Altogether, the translators had to work on six texts (two texts per task). For the (monolingual) post-editing tasks, the texts were pre-translated by Google Translate. These translation and editing sessions were recorded with an eye-tracker (Tobii TX300) and a keylogging program (Translog II).

Using MT output for translation tasks should provide advantages in efficiency and reduce research effort. In the study at hand, the research effort was compared between post-edited texts and translations from scratch to indicate whether the MT output supported the translator. Therefore, multi-word units and syntactical structures were investigated on the following parameters: quality (MT output/target text solution), duration of unit production time (Dur), and total gaze time on source text unit/target text $(\operatorname{TrtS} / \operatorname{TrtT})$.

\section{Expert and Novice Differences in Gaze Transitions during Play of a Dynamic Video Game}

\author{
Wayne D. Gray, Ryan M. Hope, John K. Lindstedt, Matthew-Donald Sangster \\ Rensselaer Polytechnic Institute, United States of America \\ grayw@rpi.edu
}

Research using dynamic video games as experimental tasks is increasingly popular; however, with rare exceptions, most such studies compare pre- and post-play scores with preand post-play changes in performance on various task batteries. In contrast, our Tetris ${ }^{\mathrm{TM}}$ studies have collected an hour's worth of play data from more than 120 players at all levels of Tetris expertise while logging all screen objects, user events, and system events. Using the Dynamic Regions of Interest (dROI) feature of GazeTool (Hope, 2014), we analyze gaze transitions among static and dynamic ROIs. Indeed, our log files enable us to see the near future so as to classify gazes to areas of the board where either the current Tetris piece or the next Tetris piece will be placed. We present the results of our analyses comparing transition differences between expert and novice Tetris players as well as differences in transitions for experts at easy versus difficult levels of game play. We also report preliminary attempts to classify players as expert or novice based on between-player differences in eye transitions. 
Posters

\title{
Effect of Expertise on the Comprehension of Plotted Functions
}

\author{
Dmitry Chumachenko, Anatoly Krichevets \\ MSU, Russian Federation \\ dmitry.chumachenko@gmail.com
}

The present study investigate the effect of domain knowledge and training on the perception of function graphs when solving mathematical tasks. We compared eye movements of 14 experts and 13 novices. The tasks were: (1) to compare coefficients of two linear functions represented by the graphs, (2) to judge whether the plot shows a function or not, (3) to judge whether the presented function is an increasing/decreasing one. Our data shows, that the average fixation duration depends on two factors: expert/novice and answer correctness. Experts showed shorter fixations when they gave a correct answer and longer fixations when they were wrong. Novices had the opposite proportion (ANOVA, $p=0,017$, $\mathrm{F}(1 ; 532)=5,726)$. The consideration of a task complexity factor showed, that the previous conclusion was true only for difficult tasks (ANOVA, $\mathrm{p}=0,05, \mathrm{~F}(1 ; 532)=3,875$ ). In simple tasks, experts had shorter fixations no matter whether they were correct or not. We argue that novices did not really solve difficult tasks; they just pretended to and tried to guess the answer, so their fixations were short. Experts on the contrary made efforts to solve difficult tasks, and their fixation duration increased. Thus, the average fixation duration indicates mental efforts. Supported by RFBR, grant No. 15-06-06319

\section{Which aspects are important to elicit gaze following in typically developing infants?}

Lise Van Schuerbeeck ${ }^{1,2}$, Marijke A.K.A. Braeken ${ }^{1,2}$, Jean Steyaert ${ }^{2,3}$, Raf Meesen ${ }^{1,4}$, Marleen Vanvuchelen ${ }^{1,2}$

${ }^{1}$ REVAL Rehabilitation Research Center - BIOMED Biomedical Research Institute, Faculty of Medicine and Life Sciences, Hasselt University, Diepenbeek, Belgium

2 Leuven Autism Research (LAuRes), KU Leuven, Leuven, Belgium

3 Department of Child Psychiatry, UPC-KU Leuven, Leuven, Belgium

4 Motor Control Laboratory, Research Center for Movement Control and Neuroplasticity, Department of Biomedical Kinesiology, Group Biomedical Sciences, KU Leuven, Heverlee, Belgium

lise.vanschuerbeeck@uhasselt.be

Introduction: This eye-tracking study aims to investigate which aspect primarily drives gaze following behavior (GFB). Particularly, we investigated the effect of eyes and of object presence on GFB.

Methods: 43 infants between 1 and 2 years of age ( $M=17.12$ months, $\mathrm{SD}=3.46)$ saw video clips of an adult turning her head to the right or left in four different conditions: openeyes condition with objects, open-eyes condition without objects, closed-eyes condition with objects and closed-eyes condition without objects. Eye movements were measured with a Tobii T120. The outcome parameters of GFB were the amount of correct first looks, a difference score for amount (amount of correct minus amount of incorrect first looks) and a difference score for looking duration (looking duration towards the correct minus looking duration towards the incorrect target).

Results: A 2x2 repeated measures ANOVA (object/no object*open/closed eyes) revealed a significant main effect for objects on the amount of correct first looks $(\mathrm{F}=59.9 ; \mathrm{p}<.001)$. No other significant main or interactions effects of the different outcome parameters were found.

Conclusion: The infants showed no difference in GFB between open and closed eyes conditions. However, infants seems to show more GFB in conditions with objects compared to conditions without objects. 


\title{
Parafoveal processing
}

\section{Co-registration of Eye Movements and Fixation-Related Potentials to Investigate Parafoveal Preview and Word Frequency Effects

\author{
Federica Degno, Piril Hepsomali, Fruzsina Soltész, Nick Donnelly, Simon P. Liversedge \\ University of Southampton, United Kingdom \\ F.Degno@soton.ac.uk
}

The time course of visual word identification has typically been investigated via eventrelated potentials (ERPs). Previous research has mainly focused on the late ERP components of the visual word identification in studies where typically one word at a time is presented. The current research uses the boundary paradigm to investigate the influence of both low-level visual and linguistic processing during natural reading. Eye movements (EMs) and ERPs were simultaneously recorded as participants read English sentences. Target word parafoveal preview (identical vs. degraded) and word frequency (low vs. high) were manipulated. Preliminary analyses of gaze durations on the target word show robust main effects of preview and frequency, demonstrating that our manipulations were extremely effective. Saccadic artefacts have been removed from the ERP record, and we are currently analysing these data with a focus on early ERP components, time-locked to fixation episodes (pre-target, target and post-target fixations). The full set of EM and ERP analyses will be discussed in relation to current theories of foveal and parafoveal processing in reading.

\section{Parafoveal preview effects of word $n+2$ in fixational eye-movements during reading}

\author{
Stefan Alexander Seelig, Sarah Risse \\ Potsdam University, Germany \\ sseelig@uni-potsdam.de
}

During reading, the perceptual span is asymmetric around fixation, being larger towards reading direction. But how do readers process the spatially distributed information available during each fixation? Fixation duration analyses constitute most of the current research. However, also the small eye-movements during each fixation may be sensitive to attention and processing demands providing a temporally more fine-grained picture of integration across the perceptual span.

The present study systematically investigated fixational eye-movements on three successive words in the boundary paradigm, manipulating parafoveal preview of word $n+2$ and lexical status of word $n+1$. Depending on the fixations' proximity to the boundary, right-eye movements on word $n$ showed subtle differences contingent on word $n+2$ preview over the course of fixation. Also word $n+1$ if fixated revealed differential fixational eye-movement trajectories with respect to being either a function- or content-word.

These results suggest that the analysis of continuous gaze positions during fixation can provide insights into the time course of parafoveal and foveal processing, probably not obtainable with fixation durations alone. Further research has to prove the cogency of this approach and the reliability of the findings regarding foveal and parafoveal processing demands showing differential effects in fixational eye-movements. 
Posters

\title{
The influence of parafoveal processing of character $\mathrm{N}+2$ on saccade targeting in Chinese reading \\ Xuejun Bai, Yongsheng Wang, Chuanli Zang, Guoli Yan \\ Tianjin Normal University, China \\ bxuejun@126.com
}

Research has shown that parafoveal processing affects saccade targeting in reading of wordbased alphabetic languages like English, however it is less clear in reading of characterbased Chinese language, in which word boundaries are not clearly demarcated. This raises a question regarding whether Chinese readers select a word or a character as their saccade target. In the present study, participants read sentences containing a two-character string in which the first $(\mathrm{N}+1)$ and second character $(\mathrm{N}+2)$ could either form a word or a nonword (two single-character words). The preview of character $\mathrm{N}+2$ was manipulated: either an identity or a pseudocharacter. The boundary paradigm was used with boundary located before character $\mathrm{N}+1$. The results showed that compared to the pseudocharacter preview, the identical preview lead to longer saccades and closer to the center of a two-character string in the word condition. However such an effect did not occur in the nonword condition. Furthermore outgoing saccades and initial landing positions on the two-character string did not differ between the word and nonword condition. These suggest that parafoveal processing from character $\mathrm{N}+2$ influences saccade target selection, but Chinese readers do not have a preference to select a word as their saccade target in reading.

\section{Brain-electric correlates of the preview benefit: An overview of findings}

\author{
Olaf Dimigen, Benthe Kornrumpf, Florian Niefind, Werner Sommer \\ Humboldt Universität zu Berlin, Germany \\ olaf.dimigen@hu-berlin.de
}

The identity preview benefit in reading - shorter fixations on parafoveally visible words - is a benchmark finding in eye-tracking research, but little is known about its neuroelectric correlates. Using fixation-related potentials (FRPs), we recently established a distinct pattern of EEG changes following fixations on previewed words, consisting of an attenuation of the occipito-temporal N1 component ("preview positivity") and the following N400 (Dimigen et al., 2012). This poster summarizes key results of several follow-up experiments investigating this effect in more detail. In all experiments, EEG and eye movements were recorded during fluent reading while preview on the upcoming word was manipulated (Boundary Paradigm). Two experiments also included a no-eye movement ERP condition in which the same materials were presented using RSVP with or without preview. We show that the preview positivity, in particular, is a reliable signature of the preview benefit that (1) is found with different types of masks, (2) in sentence and word list reading, (3) in FRPs and ERPs, (4) and alphabetic and Chinese script. Its size varies systematically according to (5) the number of previewed letters, (6) foveal load during the preceding fixation, and (7) the need to actively execute saccades (Kornrumpf et al., in prep.). 


\title{
Reading
}

\author{
Word Order and Pupillometry \\ Leigh Breakell Fernandez ${ }^{1,2}$, Barbara Hoehle ${ }^{1}$, Jon Brock ${ }^{2}$, Lyndsey Nickels ${ }^{2}$ \\ 1 University of Potsdam, Germany \\ 2 Macquarie University, Australia \\ leighfern@gmail.com
}

This study investigated the processing of canonical (SVO) and non-canonical sentence (OVS) word order in German by measuring peoples' pupil diameter change. By this, a new child-friendly method to investigate the processing costs associated with word order variation was tested. The relative order of subject and object in a sentence are relatively free in German; however OVS orders cause more processing costs in adults and are hard to understand for German children up to an age of 7 years. This is one of the first studies that used pupillometry to investigate whether the difficulties associated with OVS constructions in German are measurable with pupil diameter change. Pupil diameter and comprehension during aurally presented constructions was measured; we manipulated word order and case marking ambiguity (Table 1 ). At the disambiguating 2nd noun phrase late disambiguating OVS constructions revealed a larger pupil change $(X 2(1)=4.12, p=0.04)$ compared to early. Across the whole sentence there was larger pupil change $(X 2(1)=9.51, p=0.002)$ for OVS constructions compared to SVO, with the late conditions evoking a larger peak pupil size than the early $(\mathrm{X} 2(1)=3.71, \mathrm{p}=0.05$ (Figure 1$)$. Overall pupillometry is sensitive to the processing required in the comprehension of different word orders.

\section{In search of a pupillary optimal viewing position (POVP) \\ Patrick Hynes, Ronan Reilly \\ Maynooth University, Maynooth, Ireland, Germany phynes@cs.nuim.ie}

Previous research on isolated word recognition found words identified more rapidly when fixated near their centre. There was also reduced probability of refixations. Vitu et al. found an inversion of this effect for fixation durations in continuous reading. However, Nuthmann et al. demonstrated that this could be accounted for by misplaced fixations at the word boundaries. Our study aimed to determine whether the OVP influences pupil diameter. Given that pupil diameter is positively correlated with cognitive load, it was hypothesised that variable load in word processing should be detectable in pupil diameter as a function of landing position. Twenty two native Irish speakers were asked to read 80 sentences. Analyses were carried out on pupil diameters for first fixations on words of length 4-7. Factors such as ambient light and screen luminance were controlled. In addition, pupil diameters were corrected for increases associated with saccade size. Results showed an OVP-like curve, with a trough near the word centre. Analysis was complicated by a variation in pupil diameter over time. Nonetheless, our findings support pupillometry as an additional measure of cognitive load providing we control a range of potentially confounding factors. 
Posters

\title{
The role of context in the processing of Russian suffixed and simple words
}

\author{
Olga Nagel \\ National Research Tomsk State University, Russian Federation \\ olga.nagel2012@yandex.ru
}

The study is aimed to investigate morphological effect of Russian suffixed words in semantically congruent and incongruent context during reading. Russian simple and suffixed words of predicative (characterizing) nature were used as a material (e.g. guly-aka (reveler) vs. povesa (loafer)) to test lexicological (Blinova, 1984; Golev, 1989) and derivational interpretation of motivation (Jantsenetskaya, 1984; Rezanova, 1996; Nagel, 2005) in morphologically complex words. Lexicological approach does not differentiate processing of simple and suffixed words in the contexts actualizing inner form of the word whereas derivational approach claims primary role of morphological nature of a word in sentence functioning. The study employs eye-tracking methodology which is gaining popularity in studies on morphological processing in contextual setting (Kuperman, Bertram \& Baayen, 2010). The experiment was run using SMI 500 RED eye tracking system. Suffixed and simple nouns were embedded in sentences and were followed by either semantically congruent (gulyaka ... veselitsa (reveler ...has fun)) or semantically incongruent word (guly-aka ... chitayet (reveler... reads )). Early processing measures (first fixation, first pass) were taken to discuss non-contextual morphological effect and later processing measures (second pass, total fixation duration, $\mathrm{N}$ of regressions) were used to evaluate the effect of context.

\section{Revealing the Visual Requirements for Reading Chinese: Evidence from the Disappearing Text Paradigm}

\author{
Guoli Yan ${ }^{1}$, Nina Liu ${ }^{1}$, Lili Yu ${ }^{1}$, Kevin B. Paterson ${ }^{2}$ \\ 1 Tianjin Normal University, China \\ 2 University of Leicester, United Kingdom \\ psyygl@163.com
}

Studies using the disappearing text paradigm with alphabetic languages, such as English, show words must remain visible for as little as $60 \mathrm{~ms}$ following fixation for reading to be normal. But little is known about the time required to identify words when reading logographic languages, such as Chinese. Accordingly, we report several studies that used this paradigm to investigate the visual requirements for reading Chinese. Participants read sentences composed uniformly of two-character words, including a target word of high or low lexical frequency. Sentences were displayed normally or the fixated word (Experiment 1a \& $1 b)$, or the word to its right (Experiment 2), was visible for a limited period (20, 40, 60, 80ms) following fixation before disappearing. Disappearing text had little effect on reading rates, although readers made fewer refixations and more regressions when the fixated word disappeared, and more refixations when the word to its right disappeared, similar to findings from English. Additionally, normal word frequency effects were observed in these experiments, demonstrating that words were recognized normally even when the fixated word, or the word to its right, disappeared soon after fixation. The findings reveal the remarkable efficiency of the visual and lexical processing of text when reading Chinese. 


\title{
Long range regressive saccades in developing readers \\ Anne Friede ${ }^{1}$, Christian Vorstius ${ }^{1}$, Albrecht W. Inhoff ${ }^{2}$, Ralph Radach ${ }^{1}$ \\ ${ }^{1}$ University of Wuppertal, Germany \\ 2 State University of New York, Binghamton \\ afriede@uni-wuppertal.de
}

Skilled adult readers utilize two different strategies to locate and go back to previously fixated words, either executing an accurate long range regressive saccade or engaging in a multi-saccade backtracking strategy. Information for long range regressions is retrieved from spatial working memory (Radach \& Vorstius, 2011). The present work examined regression strategies in German 4th grade elementary school students. Participants read single line sentences containing a target word close to the beginning or end of the line. They were asked to go back and check target words for a spelling error, which was present in 50 percent of the trials. Saccade contingent line shifts were implemented to determine the contribution of spatial memory to the programming of regressions.

Results indicate much more unsystematic regression behavior as seen previously with adult readers, regardless of reading skills. The single shot frequency is far less frequent and landing position variability substantially inflated. Interestingly, developing readers also engage in an alternative strategy, where a long saccade is send to about halfway back into the sentence, followed by shorter saccades towards the target word. Based on these data, we discuss the role of spatial coding in the development of more and more automated reading skills.

\section{The effect of word spacing and word frequency for Chinese-English bilinguals during bilingual reading}

\author{
Xin Li, Xuejun Bai, Guoli Yan
}

Tianjin Normal University, People's Republic of China lixinpsy1983@126.com

For Chinese-English bilinguals, Chinese texts do not contain distinct word boundaries as English texts. We examined the effect of word spacing and word frequency on ChineseEnglish bilinguals' eye movement behavior during bilingual reading. Experiment 1 used Chinese sentences, which was 2 (word frequency: high, low) $\times 4$ (space highlighted presentations: normally unspaced, word spaced, character spaced, non-word spaced) design. Word spacing caused no facilitation or disruption for both high and low frequency words, but character spaced format could facilitate the word identification for low frequency words. Furthermore, the interference of non-word spaced format was more for low frequency words than high frequency words. Experiment 2 used English sentences, which was 2 (word frequency: high, low) $\times 3$ (spacing highlighted presentations: normally spaced, alternatinghighlighted unspaced, regular unspaced) design. There was a reading cost in the unspaced sentences relative to normally spaced sentences; this cost was much smaller in alternatinghighlighted unspaced sentences than in regular unspaced sentences. Moreover, the removal of space information increased the word frequency effect, and the disruption was more significant for low frequency words relative to high frequency words. Overall, these results indicate that the spacing effect was more significant for low frequency words during bilingual reading. 
Posters

\title{
Syntactic Ambiguity Resolution: an eye-tracking study with reference to Russian
}

\author{
Daria Chernova, Veronika Prokopenya \\ St.Petersburg State University, Russian Federation \\ chernovadasha@yandex.ru
}

Structural ambiguity resolution is a widely discussed problem in sentence processing studies (Frazier\&Rayner 1982, Traxler, Pickering, Clifton 1998, Logačev \&Vasisht 2015 etc). We consider the case of ambiguous adjunct attachment to a complex NP (I met the servant of the actress living nearby) in Russian using eye-tracking technique. Three experimental conditions are compared: globally ambiguous attachment, high attached adjunct and low attached adjunct (ambiguity resolved by means of case morphology). Early effects (firstpass time) show that high attachment structures require more time to process than low or ambiguous attachment (as Late Closure principle predicts). However, late effects (dwell time and regressions into the target region and out of it) show that adjunct attachment to a more discourse prominent NP (i.e. the head of the complex NP) is more preferable. Regressions to competing NPs also show that NP1 is reread more often. Online eye-movements data correspond to offline data - interpretations of the sentences given by participants (i.e. answers to questions who lived nearby?) - which also show strong high attachment bias. So we see two stages of sentence processing, the first one is driven by locality principles and the second one is discourse-driven.

\section{Task effect on Eye-Fixations-Related Potentials in reading}

\author{
Aline Frey ${ }^{1}$, Benoit Lemaire ${ }^{2}$, Marion Manen ${ }^{3}$, Anne Guérin-Dugué ${ }^{3}$ \\ 1 CHArt Laboratory \& ESPE of the Creteil Academy \\ 2 University Grenoble Alpes, LPNC UMR5105 - CNRS \\ ${ }^{3}$ Laboratory GIPSA-lab, University Grenoble Alpes \\ aline.frey@u-pec.fr
}

Our study examines how readers adjust their behavior according to task demands. To that end, Eye-Fixations-Related Potentials (EFRP) were registered while twenty participants read short paragraphs preceded by a title. In one task ("reading"), they had to read and then to answer to a question regarding the presence or not of a word, or the link between text and title. In the second task ("decision"), they had to decide, as quickly as possible during reading, if the text was related or not to the title.

Behavioral responses show that participants perform better the "decision" task. Ocular data (fixations, saccades, refixations, regressions ...) confirm previous findings (Carver, 1990) showing that reading process is mainly affected by reading tasks.

EFRP show that the P2 component amplitude on the first fixation on the text is larger in the "decision" vs. "reading" task, potentially reflecting working memory load (Lefebvre et al. 2005). Moreover, the P1 component amplitude in the "decision" task is larger for the first fixations and gradually decreases for the subsequent ones, whereas this gradient is not observed in the "reading" task, suggesting a modulation of the allocation of attentional resources during reading in respect of the task (Hillyard et al., 1998). 


\title{
Stroke Encoding in Simplified and Traditional Chinese Character Processing: An Eye-tracking Experiment
}

\author{
Minna M. Zhai ${ }^{1,2}$, H.C. Chen ${ }^{2}$, Michael C. W. Yip ${ }^{1}$
}

1 The Hong Kong Institute of Education, Hong Kong S.A.R. (China)

2 The Chinese University of Hong Kong, Hong Kong S.A.R. (China) mzhai@ied.edu.hk

One of the distinctive characteristics of the Chinese language is that it has two concurrent orthographies: traditional Chinese and simplified Chinese. About $30 \%$ of the simplified characters are a reduction in the number of strokes or structural complexity from traditional Chinese characters. Hence, the two orthographies are different in many important aspects including the number of strokes. Since traditional Chinese character contains more strokes than the simplified one, it is possible that it also has more redundant strokes. Therefore, the stroke encoding in character recognition might be different between traditional characters and simplified ones. The present study was conducted to examine whether traditional and simplified Chinese readers differed in stroke encoding in character processing by using the stroke removal paradigm. In the eye-tracking experiment, participants were required to read sentences comprised of characters with different proportions and types of strokes removed in order to explore whether strokes would play different roles in processing simplified and traditional characters. Results revealed that simplified Chinese readers used configuration information more efficiently for lexical access than traditional Chinese readers. Moreover, the beginning strokes were more influential than ending strokes and this difference was more salient for traditional Chinese readers than for simplified Chinese readers.

\section{The use of Theory of Mind in Pronoun Resolution: An eye-tracking study}

\author{
Patrick Sturt ${ }^{1}$, Nayoung Kwon ${ }^{2}$ \\ ${ }^{1}$ University of Edinburgh, United Kingdom \\ 2 Konkuk University, South Korea \\ patrick.sturt@ed.ac.uk
}

In comprehension, we often reason about the mental states of others, requiring Theory of Mind (ToM). We tested (a) whether ToM is used on-line in pronoun resolution, and (b) whether ToM information constrains all stages of pronoun resolution, or alternatively, whether there is an early stage of processing where ToM is ignored.

In an eye-tracking experiment, 32 participants read 40 short stories (see supplementary materials). The introductory sentence established a "real world" context, and we orthogonally manipulated (a) whether the belief of one of the protagonists was true, based on the real world context, and (b) whether the gender of a pronoun in the final sentence matched the real world context. To be globally congruent, the pronoun should match the real world context in the true belief condition, and mismatch the real world context in the false belief condition. Thus, ToM is required to compute a congruent interpretation, and this should lead to a matching_X_belief interaction at the pronoun.

There was no evidence of an early stage of processing that ignored ToM: the predicted interaction, was found in multiple measures, beginning with first-pass reading time in the pronoun region (supplementary material shows total time). 
Posters

\title{
Scan paths and scan patterns
}

\section{The evaluation of individual differences using fractal analysis of scanpaths}

\author{
Galina Y. Menshikova ${ }^{1}$, Rostislav V. Belyaev², Vladimir V. Kolesov², Victor I. Ryabenkov ${ }^{2}$ \\ 1 Lomonosov MSU, Russian Federation \\ 2 Institute of Radioengineering \& Electronics, Russian Academy of Sciences, Moscow, Russian \\ Federation \\ gmenshikova@gmail.com
}

In our previous work the method of fractal analysis was used to evaluate eye movement scanpaths (Belyaev et al., 2013). The assessment procedure was used to evaluate scanpaths fractal dimensions (Higuchi, 1988), which allowed evaluating the structural complexity of eye movements. The aim of the current study was to use the method in order to explore individual differences in eye tracking paths during fixations. Specifically we investigated whether fractal dimensions of scanpaths would change depending on external factors (an image orientation) and on internal factors (individual strategy of eye movements). 24 stimuli were presented: 3 types of images (a landscape, a sea wave image and a fractal image) each of which had 8 orientations relative to the central image point $\left(0^{\circ}, 45^{\circ}, 90^{\circ}, ..\right) .24$ participants were tested. The results showed that there were no significant differences in fractal dimension values for different image types and orientations. However, significant fractal dimension differences were revealed within the individuals. So, the method may be used to evaluate characteristics of eye movement scanpaths and to distinguish individuals accurately.

The study was supported by RFBR grant No. 13-07-00834

\section{Test of a Dynamical Model for Natural Scene Exploration Heiko H. Schütt ${ }^{1,2}$, Hans A. Trukenbrod ${ }^{1}$, Lars Rothkegel ${ }^{1}$, Ralf Engbert ${ }^{1}$ \\ 1 Universität Potsdam, Germany \\ 2 Universität Tübingen, Germany heiko.schuett@uni-potsdam.de}

Recently, we proposed a dynamical model for the generation of scanpaths during free viewing of natural scenes (Engbert et al., 2015, J Vis). Here we studied important aspects of the simulated scanpath (e.g., saccade length distributions and clustering of fixations) by comparison with a new experimental data set in combination with an alternative parameterfitting procedure. In the new experiment, participants reinspected images for a second time, which changes many aspects of the eye movement traces including statistical aggregation of fixations. We apply point process statistics and spatial correlation functions to compare experiments and model simulations. Furthermore, we investigate different methods to fit the model to data, based on a maximum likelihood approach and Bayesian Markov Chain Monte-Carlo sampling (MCMC). Our results indicate that variations of model parameters between different viewing conditions can be interpreted as strategic variations in viewing behavior. 


\section{The role of initial fixation position on subsequent gaze behavior \\ Lars Rothkegel, Hans A. Trukenbrod, Ralf Engbert \\ Universität Potsdam, Germany \\ Irothkeg@uni-potsdam.de}

Our eyes generate complex sequences of fixations during scene perception. Important predictors of fixated locations are bottom-up factors like luminance contrast, top-down factors like viewing instruction, and oculomotor biases like the tendency to place fixations near the center of an image. However, little is known about the dynamics of the scanpath after experimental manipulation of specific fixation locations. Here, we investigate the influence of the initial fixation position on subsequent eye-movement behavior on an image. We presented 64 colored photographs to participants who started their scanpath from one of two experimentally controlled positions near the right or the left border of an image. We computed the images' saliency maps and classified them as neutral images or images with high saliency values on either the left or the right side of a picture. As a result, we found that the interaction of starting position and image type had a strong impact on gaze patterns. Most importantly, the bias generated by the initial fixation position remained for several seconds. This demonstrates that target selection during scene perception is strongly influenced by fixation history. Our results emphasize the need to understand the dynamics of target selection during scene perception to explain behavioral variability. 


\section{Author Index}

A

Aagten-Murphy, David, 28

Abedian-Amiri, Ali, 193

Abegg, Mathias, 134, 140

Ablinger, Irene, 230

Acartürk, Cengiz, 111, 199, 252

Adam, Ramina, 75

Adams, Hieab H., 203

Agamennoni, Osvaldo Enrique, 53, 71

Alahyane, Nadia, 172

Aldaba, Mikel, 178, 182

Al Dahhan, Noor Z., 87

Alexeeva, Svetlana, 168

Almeida, Pedro Rocha, 231

Alotaibi, Albandari Sultan, 271

Alvarez, Tara Lynn, 148

Amit, Roy, 44

Anderson, David, 270

Anderson, Nicola C., 62

Anderson, Tim J., 54

Andersson, Richard, 44, 139

Andrews, Sally, 131

Angele, Bernhard, 34, 166

Anisimov, Victor N., 225, 248

Annac, Efsun, 215

Ansorge, Ulrich, 122, 156, 213, 221-224, 265

Antolin, Jastine, 146

Arkesteijn, Kiki, 237

Arthur, Patricia, 38

Asada, Kosuke, 227

Ashraf, Mohamed, 242

Augustynowicz, Paweł, 272

Avila, Eric, 245

Ayaya, Satsuki, 227

B

Baber, Chris, 255

Bai, Xuejun, 47, 201, 249, 276, 279

Balaj, Bibianna, 151, 260, 272

Ball, Anna, 226

Barcellos, Jéssica, 157

Barla, Michal, 195

Barrington, Rhiannon S., 78, 143

Barth, Erhardt, 120

Bartl-Pokorny, Katrin D., 167
Batten, Jonathan P., 121, 239

Beck, Valerie Margaret, 124

Becker, Stefanie I., 51

Bedell, Harold, 43

Bedford, Rachael, 121

Beken, Figen, 199

Belanger, Nathalie N., 68

Belopolsky, Artem, 112, 123, 221

Belyaev, Rostislav V., 282

Bendlin, Ewelina, 151

Benedek, Mathias, 155

Bensch, Frank, 255

Benson, Valerie, 53, 148, 225

Bente, Gary, 192

Berg, David, 72

Bertel, Sven, 155, 163, 240

Bertram, Raymond, 82, 246, 255

Bezgin, Gleb, 123

Bianchi, Bruno, 203

Bielecki, Maksymilian, 67, 263

Bieliková, Mária, 193, 195, 256

Bilalić, Merim, 154

Binda, Paola, 106

Bischof, Walter, 63

Blinnikova, Irina, 220, 266

Bloch, Carola, 192

Blythe, Hazel I., 78, 86, 102, 117, 118, 185,249

Boardman, Michael, 115

Boettcher, Sage E. P., 215

Bogacz, Rafal, 64, 171

Böhme, Hans, 149

Bolderston, Helen, 146

Boon, Paul, 123

Borah, Josh, 92, 93

Borgholt, Lasse, 207

Born, Jan, 233, 235

Born, Sabine, 103, 178

Bornkessel-Schlesewsky, Ina, 101

Borras García, Rosa, 178, 182

Botzer, Assaf, 219

Bouamama, Sana, 24

Bozkurt, Ufuk, 252

Braeken, Marijke A.K.A., 274

Braithwaite, Graham, 211 
Author Index

Brand-Gruwel, Saskia, 26

Braun, Christoph, 176

Braun, Doris I., 184

Bremmer, Frank, 181

Brett, Chris, 271

Brieber, David, 142

Brien, Donald C., 87

Brinkmann, Hanna, 141

Brock, Jon, 277

Brunstrom, Jeffrey M., 264

Buchinger, Shelley Imanuela, 265

Bulatova, Elina, 161

Bulling, Andreas, 265

Buonocore, Antimo, 107

Burggraaf, Rudolf, 51

Burr, David C., 106

C

Cabestrero, Raúl, 256

Cajar, Anke, 216

Caldara, Roberto, 43, 126, 127, 138

Carbajal, Maria J., 203

Carrillo, Facundo, 203

Carrol, Gareth T., 60, 81, 205

Caspi, Avi, 76

Casteau, Soazig, 174

Castelhano, Monica S., 148

Castro, Liliana Raquel, 53, 71

Catena, Andrés, 256, 258

Cavanagh, Patrick, 103, 190

Cave, Kyle R., 49, 128

Cerqueira, João José, 228, 229

Chang, Min, 79, 248

Chen, H.C., 281

Chen, Xiuli, 255

Chen, Yen-Chih, 201

Cheng, Haiyu, 205

Cheng, Jiayi, 213

Chernova, Daria, 199, 280

Chetverikov, Andrey, 217

Chi, Hui, 48, 201

Chiu, Kuo-Chih, 138

Choi, Soonja, 156

Cholewa, Piotr, 151

Chrobot, Nina, 156

Chua, Fook-Kee, 175

Chumachenko, Dmitry, 274

Cicchini, Guido Marco, 106

Clark, Rosie, 64

Clarke, Alasdair D. F., 50, 77
Clayden, Adam C., 217

Cleveland, Dixon C., 90-93

Collins, Thérèse, 28, 103, 172, 190

Cölln, Martin Christoph, 208

Colombo, Françoise, 43

Comesaña, Montserrat, 202

Conci, Anna, 154

Conklin, Kathy, 60, 81, 205

Cooke, Neil James, 255

Cop, Uschi, 59

Corneil, Brian, 73

Cornelissen, Tim H. W., 50, 238

Costa, João P. M., 228

Costen, Nicholas, 212

Crawford, Trevor, 125, 173, 231

Crispin, Haywood, 262

Cristino, Filipe, 209

Cruice, Madeline, 80

Cui, Jie, 198

Cui, Lei, 201

Cutter, Michael G., 101, 165

Czuchlewski, Kristina R., 25, 26

D

Dalmaijer, Edwin S., 194, 232

Daraz, Jakub, 193

da Silva, Lucas Borges, 231

Davies, Robert, 173

de Bruin, Anique, 243

Degno, Federica, 275

Dehais, Frédéric, 261

de Jeu, Marcel, 180

Dekker, Arne, 191

Demeyer, Maarten, 192

Deniz, Ozan, 252

Deubel, Heiner, 28, 64

Devereaux, Alex, 231

Diaz-Piedra, Carolina, 258

Děchtěrenko, Filip, 183, 196

Dickins, Jonathan H., 78, 109, 118

Dijkerman, Chris, 75

Dimigen, Olaf, 276

Dirix, Nicolas, 102

Di Stasi, Leandro L., 256, 258

do Lago, Olival Cardoso, 272

Domaradzka, Ewa, 263

Dominguez Martinez, Estefania, 150

Donk, Mieke, 40, 62, 221

Donnelly, Nick, 49, 114, 115, 128, 142, 214,275 
Donovan, Tim, 125, 173

Doré-Mazars, Karine, 172, 179

Dorr, Michael, 120, 182

do Santos Rodrigues, Erica, 162

Draschkow, Dejan, 115

Dreszer, Joanna, 151, 260

Drieghe, Denis, 24, 47, 59, 78, 97, 101, $110,131,165,201$

Duan, Zhaohui, 244

Duch, Włodzisław, 260

Dunn, Matt James, 187

Dupont, Lien, 136

Dussias, Paola E., 59

Duyck, Marianne, 28

Duyck, Wouter, 59, 102

Dysli, Muriel, 134

\section{E}

Ecalle, Jean, 24

Edwards, Grace, 108

Ehinger, Benedikt Valerian, 209

Ehrig, Florian, 261

Eilers, Sarah, 247

Einhäuser, Wolfgang, 61

Einspieler, Christa, 167

Elbaum, Tomer, 219

Elisa, Rebecca Nicole, 226

End, Albert, 252

Endo, Reika, 181

Engbert, Ralf, 45, 69, 135, 193, 216, 282, 283

Erichsen, Jonathan Thor, 187

Esser, Günter, 69

Ettinger, Ulrich, 223

Everling, Stefan, 75

Ewa, Domaradzka, 67

Eye Data Quality Standardisation Committee, COGAIN, 90, 93

\section{F}

Fado, Arnau, 178, 182

Fagard, Jacqueline, 172

Failing, Michel F., 133

Fal, Mehmetcan, 252

Falkowski, Andrzej, 156

Fedorova, Olga V., 248

Fernandes, Pedro Vieira, 231

Fernández, Gerardo, 53, 71

Fernandez, Leigh Breakell, 277
Fernandez Slezak, Diego, 203

Ferrand-Verdejo, Johan, 254

Ferrara, John, 36

Ferreira, Fernanda, 250

Ferreira, Marisa B., 202, 228, 229

Ferscha, Alois, 261

Fiebach, Christian Jens, 144

Fiehler, Katja, 171

Figueiredo, José, 228, 229

Filik, Ruth, 57, 84, 85, 147

Fischer, Martin H., 158

Fisher, Robert B., 217

Fitzsimmons, Gemma, 97, 204

Flueckiger, Barbara, 99

Foerster, Rebecca M., 65

Fonsova, Natalia, 177

Forster, Michael, 142

Forster, Renê, 157, 162

Foulsham, Tom, 121

Fragoso, Luane, 162

Francuz, Piotr, 272

Frens, Maarten A., 31, 51, 180, 245

Freudenburg, Zachary, 75

Frey, Aline, 280

Fricke, Melinda, 59

Friede, Anne, 200, 279

Frintrop, Simone, 223

Fröhlich, Eva, 247

Fuchs, Isabella, 221

Fudali-Czyż, Agnieszka, 272

Fuhl, Wolfgang, 141

Furlan, Michele, 176

G

Gagl, Benjamin, 130, 143, 144

Gamer, Matthias, 252

García-Blanco, Ana, 146

Garner, Matthew, 114, 214

Gartus, Andreas, 189

Gautier, Josselin, 43

Ge, Cao, 219

Gegenfurtner, Karl R., 29, 184

Gerjets, Peter, 235

Germeys, Filip, 192

Gerstenberger, Kerstin, 144

Geyer, Thomas, 215, 216

Ghose, Tandra, 159, 244

Gilchrist, Iain D., 30, 64, 171, 218, 242, 264

Glaholt, Mackenzie G., 38 


\section{Author Index}

Godwin, Hayward J., 49, 114, 115, 128, 204,214

Goller, Florian, 156

Goller, Jürgen, 206

Gonzalez-Rodriguez, Jose, 41

Goraczewski, Łukasz, 151, 260

Gorgas, Anna-Maria, 241, 270

Gouw, Paula, 26

Graf, Mario, 154

Gray, Wayne D., 136, 273

Greaves, Matthew, 211

Greenberg, Robert, 76

Gregg, Julie M., 94, 110

Gregory, Nicola Jean, 146

Gremmler, Svenja, 147, 241

Groner, Marina, 63

Groner, Rudolf, 99

Guan, Ying-Hua, 23

Gucma, Maciej, 116

Guérin-Dugué, Anne, 24, 210, 280

Guitton, Daniel, 76

Günther, Thomas, 79

Guo, Cindy Xiaopeng, 196

Guo, Kun, 207

Gut, Małgorzata, 151

Guyader, Nathalie, 210

\section{$\mathbf{H}$}

Haass, Michael J., 25

Hadwin, Julie A., 114, 214, 225

Hafed, Ziad, 73

Häikiö, Tuomo, 82, 109, 119, 246

Hamborg, Kai-Christoph, 259, 269

Hamel, Kate, 270

Hansen, Dan Witzner, 44

Hansen, John Paulin, 149

Harezlak, Katarzyna, 32

Harland, Beth, 142

Harris, Julie M., 262

Hartmann, Matthias, 158

Hartsuiker, Robert, 59

Harvey, Hannah, 204

Haselhuhn, Tristan, 243

Hawelka, Stefan, 70, 100, 130, 143-145

He, Liyuan, 166

Hedge, Craig, 132

Heed, Tobias, 237

Heeman, Jessica, 56

Heinen, Stephen, 183

Helle, Laura, 255
Henein, Simon, 65

Henry, Regina, 251

Henschke, Sebastian F., 234

Henz, Galina, 268

Hepsomali, Piril, 275

Hermena, Ehab W., 24, 110, 131

Herwig, Arvid, 104, 107, 190

Hessels, Roy S., 238

Heuer, Sabine, 154

Hickey, Clayton, 113

Hill, Elisabeth, 69

Hilo, Rinat, 173

Hinde, Stephen John, 30

Hodgson, Timothy, 41

Hoehle, Barbara, 277

Höfler, Margit, 218, 242

Holliman, Nick S., 49

Hollingworth, Andrew, 124

Holmberg, Nils, 98

Holmes, Paul, 212

Holmes, Timothy Paul, 262

Holmqvist, Kenneth, 41, 67, 91, 92, 98, $139,196,250$

Holtmann, Martin, 226

Holubcova, Alena, 157

Hooge, Ignace T. C., 44, 51, 139, 238

Hope, Ryan M., 136, 273

Hörmann, Stefan, 182

Horstmann, Gernot, 222

Hou, Xiujuan, 244

Howard, Philippa Lucy, 53, 148

Howes, Andrew, 255

Howman, Hannah, 85

Hsiao, Yi-ting, 48

Hsieh, Grace M. Y., 163, 164, 194

Hsieh, Tsuei-Ju, 201

Hsu, Hsien-Yuan, 23

Huber-Huber, Christoph, 213

Huck, Anneline, 80

Huestegge, Lynn, 55, 105

Humphris, Gerry, 160

Hunt, Amelia R., 50, 77

Husain, Masud, 232

Hutchison, R. Matthew, 75

Hutson, John P., 120

Hutzler, Florian, 70, 100, 130, 143-145

Hynes, Patrick, 277

Hyönä, Jukka, 17, 82, 109, 246

I

Ichi-nohe, Susumu, 257 
Ilg, Uwe, 268, 269

Inhoff, Albrecht W., 94, 110, 130, 279

Inoue, Kazutaka, 257

Iseli, Christian, 99

Isik, Berna M., 31, 180

Ison, Matias J., 88

Itti, Laurent, 19, 72

Izmalkova, Anna, 220, 266

$\mathbf{J}$

Jackel, Lina, 223

Jainta, Stephanie, 82, 102, 185

Jakesch, Martina, 264

Jarodzka, Halszka, 26, 41

Järvikivi, Juhani, 169

Jarvstad, Andreas, 171, 264

Jaschinski, Wolfgang, 185, 186

Jayes, Lewis T., 86

Jens R., Helmert, 191

Jian, Yu-Cin, 168

Jóhannesson, Ómar I., 217

Johansson, Roger, 67

Johnson, Aaron P., 211

Johnston, Kevin, 75

Jonassen, Morten, 149

Jones, Manon, 81

Joos, Markus, 93, 149

Joos, Roland E., 186

José, Ossandón, 209

Joseph, Holly, 70

Juhasz, Barbara, 95

Jurcinska, Inga, 167

\section{K}

Kaakinen, Johanna K., 84, 85, 255

Kaar, Marcus, 265

Kahl, Anna Lena, 268

Kaisler, Raphaela E., 254

Kaldos, Janos, 164

Kamide, Yuki, 56

Kamienkowski, Juan E., 88, 203

Kaminiarz, Andre, 181

Kanoh, Shin'ichiro, 257

Kao, Gloria Yi-Ming, 98

Kasneci, Enkelejda, 141

Kaspar, Kai, 192, 208, 259, 269

Kasprowski, Pawel, 32

Kato, Masaharu, 227

Kato, Takaaki, 165

Kaunitz, Lisandro N., 88
Kawashima, Ryuta, 257

Kemner, Chantal, 238

Kennedy, Colin R., 78, 118

Keresztes, Attila, 234

Kerzel, Dirk, 178

Khalid, Shah, 223

Khan, Arif, 265

Kim, Kayeon, 89

Kim, Young-Suk, 23

Kingstone, Alan, 253

Kirby, John R., 87

Kirkby, Julie A., 78, 143, 251

Kirkici, Bilal, 199

Kirtley, Clare, 124

Kiseleva, Anna S., 248

Klapetek, Anna, 64

Klassen, Robert M., 27

Klein, Angela Ines, 69

Klerke, Sigrid, 207

Kliegl, Reinhold, 34

Klimova, Oxana, 270

Knott, Lauren, 125

Knox, Paul C., 172

Koch, Iring, 55

Kocián, Matěj, 183

Kok, Ellen, 243

Kolesov, Vladimir V., 282

Köller, Olaf, 66

König, Peter, 10, 177, 208, 209, 237

Korinth, Sebastian Peter, 144

Körner, Christof, 155, 218, 242

Kornrumpf, Benthe, 276

Köster, Moritz, 259, 269

Koun, Eric, 254

Kovalev, Artem, 195, 270

Kowalski, Maciej, 151

Kramer, Arthur F., 211

Kretzschmar, Franziska, 101

Krichevets, Anatoly, 37, 274

Krieber, Magdalena, 167

Kristjánsson, Árni, 217

Kroll, Judith F., 59

Kronbichler, Martin, 100

Krügel, André, 69

Krüger, Hannah M., 103

Krumina, Gunta, 167, 186

Ku, Yu Min, 162

Kübler, Thomas, 141

Kuhn, Gustav, 69, 239

Kukona, Anuenue, 56 


\section{Author Index}

Kumagaya, Shinichiro, 227

Kummer, Claudia, 257

Kuniecki, Michał, 267

Kuperman, Victor, 22, 170, 251

Kusch, Kerstin, 191, 208

Kwon, Nayoung, 281

Kyröläinen, Aki-Juhani, 169

L

Ladstätter, Stefan, 197

Laicane, Ilze, 167

Laidlaw, Kaitlin E. W., 253

Laishley, Abby E., 143, 251

Lamers, Monique, 238

Landwehr, Niels, 93

Lange, Elke B., 198, 234

Lange-Küttner, Chris, 204

Lantz, Laura M. T., 169

Lao, Junpeng, 43, 126, 127, 138

Laou, Philips, 38

Lappe, Markus, 147, 241

Larson, Adam M., 120

Latanov, Alexander V., 225, 248

Latif, Nida, 148

Latorella, Kara, 93

Latoschik, Marc Erich, 192

Laubrock, Jochen, 117, 129, 216, 246

Leder, Helmut, 142, 189, 206, 254, 264, 267

Lee, Chia-Ying, 200

Lee, Choongkil, 89

Lee, Donghoon, 156

Leek, E. Charles, 209

Legenbauer, Tanja, 226

Leijten, Frans, 75

Leinenger, Mallorie, 83

Lemaire, Benoit, 24, 280

Lemoine-Lardennois, Christelle, 172, 179

Lencer, Rebekka, 147

Le Pelley, Mike E., 133

Leppink, Jimmie, 243

Leuthold, Hartmut, 84

Levy, Roger, 83

Lewandowska, Monika, 260

Lewkowicz, Rafał, 272

Li, Li, 31

Li, Lin, 47, 79, 248

Li, Sha, 47, 79, 248

Li, Wen-Chin, 211
Li, Xin, 279

Li, Xingshan, 46, 109

Liang, Feifei, 249

Licka, Theresia, 265

Liebscher, Daniel, 149

Lijewska, Agnieszka, 160

Lim, Joo-Hwee, 210

Lin, Horng-Horng, 138

Lin, John J. H., 150, 194

Lin, Sunny S. J., 150, 163, 164, 194

Lindner, Marlit Annalena, 66

Lindstedt, John K., 273

Linnell, Karina J., 236

Litchfield, Damien, 42, 125, 173

Liu, Huashan, 244

Liu, Nina, 166, 278

Liu, Yingdi, 43, 127

Liversedge, Simon P., 24, 47-49, 53, 78, $81,82,86,96,101,102,109$, $110,114,115,117,118,131$, $142,165,185,201,204,214$, $249,251,275$

Loertscher, Miriam Laura, 99

Lomtatidze, Olga, 161

López, Jose A., 256

Lopez García, Elena, 178, 182

Loschky, Lester C., 120, 211

Louwen, Suzanne C., 203

Lowenhoff, Alice Georgina, 262

Lüdtke, Jana, 247

Ludwig, Casimir J.H., 132, 171, 188

Lukavský, Jiří, 183, 196

Lüll, Svenja, 101

Lund, Haakon, 149

Lundbom, Nina, 255

Luniakova, Elizaveta, 195

Lunn, Judith Fiona, 173

Lüttke, Thomas, 200, 268

Lutz, Otto Hans-Martin, 260

M

MacAskill, Michael R., 54

Macdonald, Ross G., 158, 265

Macedo, António Filipe, 202, 228, 229

Machado, Liana, 271

MacInnes, W. Joseph, 57

Mack, David, 268, 269

Mackenzie, Andrew Kerr, 262

Macknik, Stephen L., 198, 256

Madsen, Janus Askø, 149 
Maggia, Christophe, 210

Magliano, Joseph P., 120

Majewski, Jakub, 151

Majka, Alicja, 151

Mancheva, Lyuba, 24

Manen, Marion, 280

Mann, Carl M., 115

Margaretha, Suzy, 245

Marin, Manuela M., 254

Marschik, Peter B., 167

Marshall, Jane, 80

Marta, Zakrzewska, 67

Martin Christoph, Cölln, 191

Martinez-Conde, Susana, 198, 256

Marx, Christina, 70, 130

Mast, Fred W., 99

Mathôt, Sebastiaan, 29, 113, 194

Matscheko, Michael, 261

Matsuki, Kazunaga, 22

Matulewski, Jacek, 151, 260

Matzen, Laura E., 25

Maxfield, Justin, 62

McCamy, Michael B., 256, 258

McCloy, Rachel, 133, 263

McCormick, Sheree Ann, 212

McGowan, Victoria A., 35, 39, 79, 248

McIntosh, Anthony R., 123

McIntyre, Nora Ann, 27

McMichael, Stephanie N., 25

McNamara, Laura A., 25, 26

McSorley, Eugene, 133, 263

Meermeier, Annegret, 147

Meesen, Raf, 274

Meeter, Martijn, 62

Megardon, Geoffrey, 188

Meghanathan, Radha Nila, 233

Mehrpouya, Hadi, 213

Meixner, Johannes M., 117, 246

Melcher, David P., 107, 253

Melmi, Jean-Baptiste, 113

Meng, Zhu, 48, 166

Menneer, Tamaryn, 49, 128

Mennel, Pierre, 99

Menshikova, Galina Y., 195, 282

Mestry, Natalie, 128

Meunier, Martine, 254

Meyberg, Susann, 45

Mezö, Charlotte, 269

Micai, Martina, 70

Michiels, Bart, 192
Miellet, Sébastien, 43, 126, 127, 138

Miguel, Olga Joana, 228, 229

Mink, Maurice, 216

Missal, Marcus, 32

Mitrovic, Aleksandra, 206, 267

Moehler, Tobias, 171

Moiseeva, Victoria, 177

Monasso, Giulietta S., 31, 180

Monika, Markowska, 204

Moore, Tirin, 20

Morar, Natan Sorin, 255

Moro, Robert, 193, 256

Morrone, M. Concetta, 106

Muckli, Lars, 108

Muczynski, Bartosz, 116

Muhl-Richardson, Alex, 214

Müller, Hermann J., 215, 216

Mulvey, Fiona, 91

Mulvey, Fiona B., 90-93

Munoz, Douglas P., 72, 87

Murris, Jozua, 221

Myall, Daniel J., 54

Myslín, Mark, 83

$\mathbf{N}$

Nadal, Marcos, 142

Nagel, Olga, 278

Nakaura, Yoshiya, 239

Narayan, Vikram, 33

Neuhuber, Norah, 40

Nickels, Lyndsey, 277

Niefind, Florian, 276

Niehorster, Diederick C., 31, 41

Nijboer, Tanja C.W., 56

Nikolaev, Andrey R., 88, 233

Nikolova, Mirela, 82, 102, 185

Nissens, Tom, 133

Nitzke, Jean, 273

Nordholt, Sontje, 208

Nottbusch, Guido, 35

Nowakowska, Anna D. F., 77

Nunnendorf, Vanessa, 177

Nuthmann, Antje, 61, 217

Nyström, Marcus, 44, 139, 250

O

O'Driscoll, Gillian A., 52

Ohl, Sven, 108

Öhlschläger, Sabine, 214

Oliva, Manuel, 41, 250 


\section{Author Index}

Oliveira, Helena, 202

Olivers, Chris, 112

Olkoniemi, Henri, 85

Ondategui Parra, Juan Carlos, 178, 182

Ooms, Kristien, 136

Orozco, David, 53

Ort, Eduard, 194

Ossandón, José Pablo, 237

Ostapchuk, Nicholas, 52

Ouyang, Xinyi, 127

Ozger, Murat, 41

\section{$\mathbf{P}$}

Paeye, Celine, 190

Pagán, Ascensión, 109, 117

Paiva, Tiago Oliveira, 231

Pajkossy, Péter, 234

Paletta, Lucas, 40, 197, 259

Pan, Jinger, 129

Paoletti, Davide, 176

Papinutto, Michael, 126

Parente, Fabio, 57

Parise, Eugenio, 150

Park, Soon Young, 140

Parreira, Marta, 228, 229

Parris, Benjamin A., 226

Pascoe, Jeffrey, 36

Paterson, Kevin B., 35, 39, 47, 79, 86, 169, 248, 249, 278

Patrone, Aniello Raffaele, 224

Pauly, Dennis, 35

Pavlou, Katerina, 225

Pelisson, Denis, 254

Peltoniemi, Suvi, 84

Pelz, Jeff, 91

Pelz, Jeff B., 90-93

Perea, Manuel, 146

Pereira, Paulo A., 228, 229

Perko, Roland, 259

Pernet, Cyril, 138

Peters, Katharina, 79

Peysakhovich, Vsevolod, 261

Pfeiffer, Thies, 116

Pfeiffer-Leßmann, Nadine, 116

Pfeuffer, Christina Ursula, 105

Pieczykolan, Aleksandra, 55

Pilarczyk, Joanna, 267

Pinke, Melissa, 154

Pinnow, Marlies, 226

Pipelidis, Georgios, 261
Pitcher, Toni L., 54

Pivneva, Irina, 58

Pniewski, Benjamin, 226

Poffa, Remo, 186

Pointner, Mathias, 215

Pokorny, Florian B., 167

Porretta, Vincent, 169

Potapchuk, Elena, 183

Poth, Christian H., 104

Potter, Douglas D., 89

Prammer, Carina, 241

Priamikov, Alexander, 33

Prokopenya, Veronika, 199, 280

Pszeida, Martin, 197

Pujol Ramo, Jaume, 178, 182

Puntiroli, Michael, 178

Q

Qu, Qingqing, 46, 109

Quiroga, Rodrigo Quian, 88

Quirós, Pilar, 256

\section{$\mathbf{R}$}

Racsmány, Mihály, 234

Radach, Ralph, 23, 79, 110, 130, 200, 230, 268,279

Ralph-Nearman, Christina, 147

Ramos Gameiro, Ricardo, 208

Ramsey, Nick, 75

Rath-Wilson, Kate, 76

Rayner, Keith, 34, 68, 83, 148

Reichelt, Maria, 163

Reichle, Erik D., 49, 96

Reid, Vincent, 150

Reilly, Ronan, 130, 200, 277

Reingold, Eyal M., 37

Renner, Patrick, 116

Rerko, Laura, 234

Retell, James D., 51

Richlan, Fabio, 100, 145

Richoz, Anne-Raphaëlle, 127

Richter, Tobias, 247

Rieiro, Hector, 258

Riggs, Charlotte A., 115

Rigoll, Gerhard, 182

Ringer, Ryan C., 211

Ríos, Francisco, 256

Risse, Sarah, 69, 129, 275

Robben, Simon, 243

Rodrigues, Erica dos Santos, 157 
Rogers, Peter J., 264

Röhrbein, Florian, 207, 242

Rolfs, Martin, 108

Rosenberg, Raphael, 141

Rosenstiel, Wolfgang, 141

Roth, Daniel, 192

Rothkegel, Lars, 282, 283

Rothkopf, Constantin, 177

Rothwell, Austin, 253

Rotova, Natalia, 266

Roy, Arup, 76

Roy, Penny, 80

Ruff, Stefan, 260

Ruffieux, Nicolas, 43

Rüger, Wolfgang, 149

Rüth, Marco, 259

Ryabenkov, Victor I., 282

Ryan, Jennifer D., 123

\section{S}

Saez, Carolina, 256

Safran, Avinoam B., 76

Sahel, Jose A., 76

Sahraie, Arash, 77

Saldaña, David, 70

Salmerón, Ladislao, 146

Sandberg, Helena, 98

Sangi, Mehrdad, 196

Sangster, Matthew-Donald, 273

Saunders, Daniel R., 253

Savage, Steven W., 89

Scannella, Sébastien, 261

Schaper, Marie Luisa, 206

Scharinger, Christian, 235

Scharke, Wolfgang, 79

Scheepers, Christoph, 250

Scheiter, Katharina, 250

Schelske, Yannik T. H., 159, 244

Schenk, Simon, 182

Scherzer, Otmar, 224

Schlagbauer, Bernhard, 216

Schlesewsky, Matthias, 101

Schmid, Sebastian, 243

Schmidtke, Daniel, 170

Schneeweiß, Paul, 216

Schneider, Michael, 207

Schneider, Werner X., 65, 104, 107

Schöberl, Tobias, 222

Schor, Paulo, 272

Schotter, Elizabeth R., 68, 96
Schreij, Daniel, 194

Schroeder, Sascha, 109, 118, 247

Schubert, Torsten, 188

Schüler, Felix, 177

Schumacher, Marcela, 53

Schuster, Sarah, 70, 100, 130, 143, 145

Schütt, Heiko H., 282

Schütz, Alexander C., 104, 112, 175, 184

Schwarz, Michael, 40, 197, 259

Schwarz, Norbert, 156

Schwedes, Charlotte, 126

Schwenzfeier, Anne Kathrin, 226

Schwertner, Emilia, 267

Sebastian, Pannasch, 191

Seelig, Stefan Alexander, 275

Seixas, Maria João de Sousa, 231

Seppälä, Katri, 121

Seya, Yasuhiro, 239

Seywerth, Raphael, 122

Shalom, Diego E., 203

Shanidze, Natela, 183

Shen, Kelly, 123

Shen, Wei, 46, 109

Sheridan, Heather, 37

Shi, Bertram, 33

Shillcock, Richard, 48, 66, 213

Shinoda, Hiroyuki, 239

Shioya, Shunsuke, 257

Shulgovskiy, Valeriy V., 177, 225

Shvarts, Anna, 37

Siebold, Alisha, 40

Sigman, Mariano, 88, 203

Silberling, Valentine, 230

Silling, Karen, 147

Silvis, Jeroen, 221

Sim, Grace, 38

Šimek, Miroslav, 195

Simko, Jakub, 256

Simonsen, Peter, 207

Simpson, Sol, 91, 93

Sinn, Petra, 45, 198

Sippel, Katrin, 141

Siu, Wilfred W.F., 31

Skilters, Jurgis, 167

Skinner, Andy, 132

Slattery, Timothy J., 34, 95

Slavutskaya, Maria, 177

Smith, Alastair D., 57, 271

Smith, Andrew T., 176

Smith, Irene J., 217 


\section{Author Index}

Smith, Sarah, 115

Smith, Tim J., 30, 120, 121, 239

Soares, Ana Paula, 202

Sokhn, Nayla, 138

Solman, Grayden, 63

Soltan, Marina, 39

Soltész, Fruzsina, 275

Sommer, Werner, 45, 276

Sousa, Inês, 228, 229

Sperlich, Anja, 117, 246

Spichtig, Alexandra N., 36

Spiegel, Simon, 99

Spiel, Katharina, 240

Spotorno, Sara, 218

Starke, Sandra Dorothee, 255

Staudte, Maria, 158

Steiner, Ingmar, 265

Steinmann, Daniel, 206

Stevens-Adams, Susan M., 25

Steyaert, Jean, 274

Stothart, George, 132

Stracuzzi, David, 26

Strandvall, Tommy, 150, 156

Stróżak, Paweł, 272

Strukelj, Alexander, 250

Sturt, Patrick, 281

Suárez, Juan, 258

Sugano, Yusuke, 265

Sumner, Emma, 69

Sumner, Petroc, 188

Suzuki, Takeshi, 244

Svede, Aiga, 186

Syed, Zeeshan, 236

Szinte, Martin, 28

\section{T}

Tagu, Jérôme, 179

Tai, Sheng Yao, 162

Tailhefer, Coline, 172

Takacs, Jadwiga, 189

Takahashi, Kohske, 189

Talanina, Aleksandra, 199

Talasan, Henry, 148

Tao, Chen-Chao, 97

Tatler, Benjamin W., 89, 124, 218

Tereshchenko, Leonid Victorovich, 225

Tew, Oliver, 114

Thakkar, Katharine N., 232

Thalwitzer, Mario, 141

Theeuwes, Jan, 123, 133
Thierry, Guillaume, 81

Thompson, Benjamin, 196

Thompson, Dominic, 250

Thompson, Robin, 80

Thornton, Ian M., 217

Throneburg, Zachary W., 211

Tiemeier, Henning W., 203

Tiffin-Richards, Simon Patrick, 118

Tinio, Pablo, 267

Titone, Debra A., 52, 58

Tousley, Elias, 36

Toyoshima, Hisashi, 181

Traub, Franziska, 67

Treija, Elina, 186

Triesch, Jochen, 33

Trukenbrod, Hans A., 135, 193, 282, 283

Truszczyński, Olaf, 272

Tsai, Jie-Li, 200

Tsotsos, John K., 12

Tudge, Luke, 188

Turcan, Alexandra, 85

Turuwhenua, Jason, 196

Tvarozek, Jozef, 256

$\mathbf{U}$

Ueda, Hiroshi, 189

Underwood, Geoffrey, 271

Uschi, Cop, 102

V

Valdois, Sylviane, 24

Valsecchi, Matteo, 29

Valuch, Christian, 122, 224, 265

Van Assche, Eva, 59

van der Geest, Jos N., 31, 51, 180, 203, 245

van der Linden, Lotje, 29, 113, 174, 194

Van der Stigchel, Stefan, 56, 75, 113, 194, 232

Van der Stoep, Nathan, 56

van der Vliet, Rick, 203

Van Dyke, Julie A., 22, 170, 251

Van Eetvelde, Veerle, 136

van Gog, Tamara, 42, 159

van Leeuwen, Cees, 88, 233

van Marlen, Tim, 159

Van Meeuwen, Ludo, 26

van Merriënboer, Jeroen, 243

Van Schuerbeeck, Lise, 274

Vansteensel, Mariska, 75 
Vanvuchelen, Marleen, 274

van Wermeskerken, Margot, 42, 159

van Zoest, Wieske, 40, 113, 132, 176, 253

Varatharajah, Alexander, 88

Vardi, Ilan, 65

Vaughan-Evans, Awel Hydref, 81

Veldre, Aaron, 131

Venini, Dustin, 51

Venjakob, Antje Christine, 260

Vennekötter, Alina, 269

Verfaillie, Karl, 192

Verghese, Preeti, 183

Vergilino-Perez, Dorine, 179

Vetter, Petra, 108

Vig, Eleonora, 120

Vitu, Françoise, 15, 29, 174

Võ, Melissa Le-Hoa, 50, 115, 137, 214, 215

von der Malsburg, Titus, 166

von Mühlenen, Adrian, 206

Vorstius, Christian, 23, 130, 200, 268, 279

Vulchanova, Mila, 70

W

Wade, Kimberly, 206

Wagner, Michael, 219

Walcher, Sonja, 155

Walker, Robin, 14, 174, 176, 204

Wang, Aiping, 60

Wang, Chin-An, 72

Wang, Dong, 91, 93

Wang, Fuxing, 240, 244

Wang, Jingxin, 47, 79, 248

Wang, Jing-Yi, 233

Wang, Mengsi, 109

Wang, Ruimeng, 219, 230

Wang, Yang, 219, 230

Wang, Yongsheng, 47, 249, 276

Wardhani, Intan, 51

Warrington, Kayleigh L., 35, 39

Wase, Cara, 66

Watanabe, Katsumi, 189

Watanabe, Masayuki, 54

Weal, Mark J., 97

Weaver, Matthew D., 40, 113

Weber, Frederik D., 233, 235

Weibel, David, 99

Weichselbaum, Hanna, 221

Weiß, Katharina, 107, 190

Wende, Kim, 32
Wentura, Dirk, 126

Wenzlaff, Frederike, 191

Wetzel, Stefanie, 155, 163

Wexler, Mark, 28

White, Brian J., 72

White, Sarah J., 35, 39, 169, 248

Whitford, Veronica M., 52, 58

Wilbers, Anne-Kathrin, 192, 269

Wild, Klaus-Peter, 243

Williams, Glenn Patrick, 56

Williams, Louis, 263

Willmes, Klaus, 230

Wolf, Christian, 104, 112

Wolfe, Jeremy M., 51, 137

Wolohan, Felicity Denise Angela, 172

Womelsdorf, Thilo, 74

Worf, Lucy C., 143

Wu, Esther Xiu Wen, 175

Wu, Jie, 205

Wu, Mengxuan, 205

$\mathrm{X}$

Xie, Fang, 79, 248

Xie, Heping, 240

Y

Yalcin, Özge Nilay, 111

Yamaguchi, Yasushi, 189

Yamanoi, Takahiro, 181

Yan, Guoli, 47, 48, 166, 201, 249, 276, 278, 279

Yan, Ming, 60, 129

Yang, Haibo, 219, 230

Yen, Shih-Cheng, 175

Yip, Michael C. W., 281

Yonemura, Tomoko, 181

Yu, Chen-Ping, 62

Yu, Chung-San, 211

Yu, Lili, 278

Yuval-Greenberg, Shlomit, 44, 173

$\mathbf{Z}$

Zander, Steffi, 163

Zang, Chuanli, 47, 48, 249, 276

Zelinsky, Gregory, 62, 174

Zemblys, Raimondas, 91, 92

Zeng, you jia, 97

Zerr, Paul, 232

Zhai, Minna M., 281

Zhang, Manman, 249 
Author Index

Zhang, Yi, 219, 230

Zimmermann, Julia, 175

Zhao, Xin, 219, 230

Zhou, Yuefang, 160

Zinno, Angela, 36 
